\title{
A SUMMARY DOCUMENTATION OF THE AMIP MODELS
}

\author{
by
}

Thomas J. Phillips

Program for Climate Model Diagnosis and Intercomparison Lawrence Livermore National Laboratory, Livermore, CA, USA

April 1994

PROGRAM FOR CLIMATE MODEL DIAGNOSIS AND INTERCOMPARISON UNIVERSITY OF CALIFORNIA, LAWRENCE LIVERMORE NATIONAL LABORATORY LIVERMORE, CA 94550 


\section{DISCLAIMER}

This document was prepared as an account of work sponsored by an agency of the United States Government. Neither the United States Government nor the University of California nor any of their employees, makes any warranty, express or implied, or assumes any legal liability or responsibility for the accuracy, completeness, or usefulness of any information, apparatus, product, or process disclosed, or represents that its use would not infringe privately owned rights. Reference herein to any specific commercial products, process, or service by trade name, trademark, manufacturer, or otherwise, does not necessarily constitute or imply its endorsement, recommendation, or favoring by the United States Government or the University of California. The views and opinions of authors expressed herein do not necessarily state or reflect those of the United States Government or the University of California, and shall not be used for advertising or product endorsement purposes.

This is an informal report intended primarily for internal or limited external distribution. The opinions and conclusions stated are those of the author and may or may not be those of the Laboratory.

This report has been repoduced directly from the best available copy.

Available to DOE and DOE contractors from the Office of Scientific and Technical Information

P.O. Box 62, Oak Ridge, TN 37831

Prices available from (615) 576-8401, FTS 626-8401

Available to the public from the National Technical Information Service

U.S. Department of Commerce

5285 Port Royal Rd., Springfield, VA 22161 


\section{DISCLAIMER}

Portions of this document may be illegible in electronic image products. Images are produced from the best available original document. 


\section{A SUMMARY DOCUMENTATION OF THE AMIP MODELS}

by

Thomas J. Phillips

Program for Climate Model Diagnosis and Intercomparison

Lawrence Livermore National Laboratory, Livermore, CA, USA

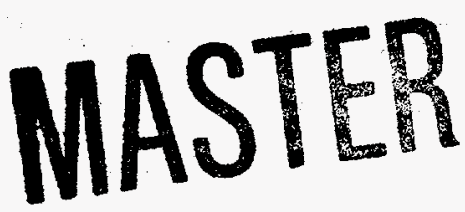

April 1994 


\section{Preface}

This summary documentation for the Atmospheric Model Intercomparison Project (AMIP) centralizes information on the features of most of today's global atmospheric models. The production of this report would not have been possible without the cooperation of representatives of the AMIP modeling groups, who supplied numerous details on model algorithms and repeatedly corrected errors that were present in earlier drafts. Thus, much collective effort was invested to ensure the accuracy of this summary documentation.

It must be acknowledged, however, that an undertaking of this magnitude and complexity is unlikely to be entirely free of error. Moreover, there will be a need to document future model entries to the AMIP. Hence, it probably will be necessary to issue later a revised summary documentation that reflects subsequent corrections, additions, and clarifications. This future model documentation will be electronic in form, since this medium affords the means for readily updating, disseminating, and accessing information. In addition, it is anticipated that this information will be distributed in a "hypertext" software format that will permit the user to selectively view the description of a particular AMIP model or a particular feature displayed for all of the models. Further information on the hypertext model documentation will be provided to AMIP participants at a later time, pending further technical developments.

In the meantime, readers are encouraged to report any errors or inconsistencies in the present summary documentation to the author at:

Program for Climate Model Diagnosis and Intercomparison (PCMDI)

Lawrence Livermore National Laboratory

L-264, P.O. Box 808

Livermore, California (USA) 94550

Internet e-mail: phillips@tworks.llnl.gov.

Phone: +1-510-422-0072

Fax: +1-510-422-7675 


\section{Table of Contents}

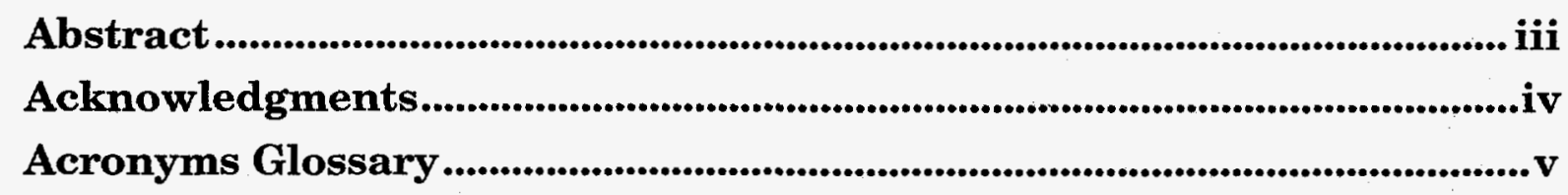

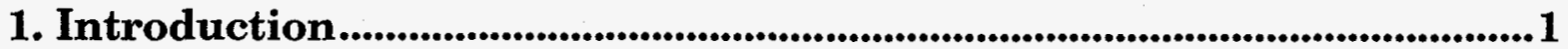

Table 1-1: AMIP modeling groups ..........................................................2

Table 1-2: AMIP diagnostic subprojects .....................................................

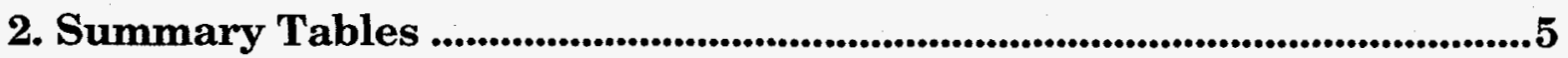

Table 2-1: Model representation/resolution ...........................................6

Table 2-2: Computational information ..................................................



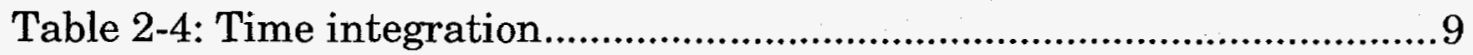

Table 2-5: Filtering, smoothing, and filling..............................................10

Table 2-6: Diffusion and gravity-wave drag ................................................11

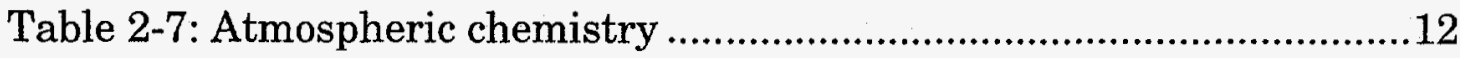

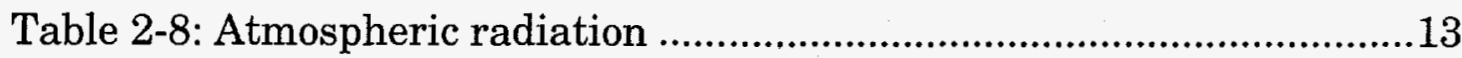

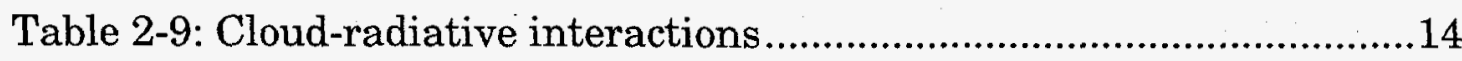

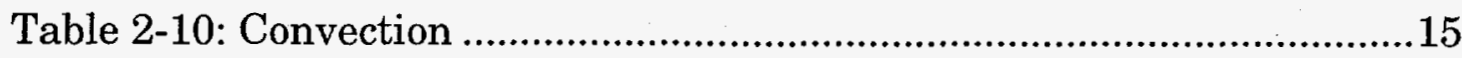

Table 2-11: Cloud formation and precipitation .........................................16

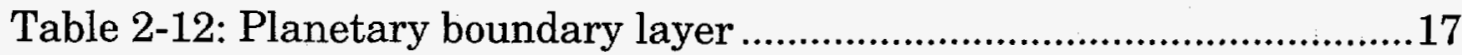

Table 2-13: Snow cover and sea ice........................................................18

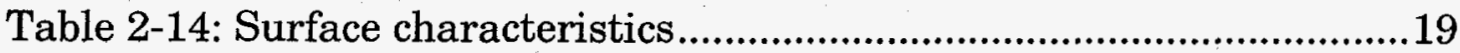

Table 2-15: Land surface processes ..........................................................20

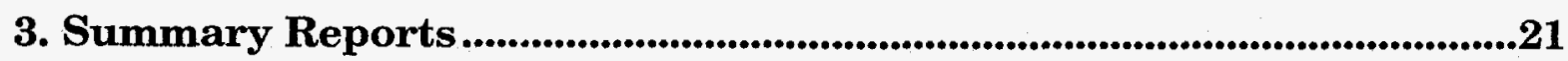

BMRC: Bureau of Meteorology Research Centre.......................................23

CCC: Canadian Centre for Climate Research...........................................31

CNRM: Centre National de Recherches Météorologiques..............................40

COLA: Center for Ocean-Land-Atmosphere Studies ....................................48

CSIRO: Commonwealth Scientific and Industrial

Research Organization 


\section{Table of Contents (continued)}

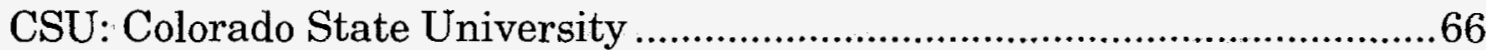

DERF: Dynamical Extended Range Forecasting ........................................

DNM: Department of Numerical Mathematics............................................83

ECMWF: European Centre for Medium-Range

Weather Forecasts

GFDL: Geophysical Fluid Dynamics Laboratory ……................................100

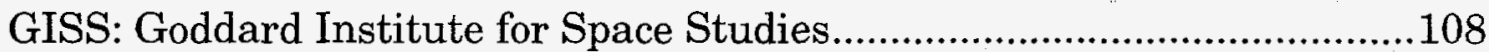

GLA: Goddard Laboratory for Atmospheres ...........................................118

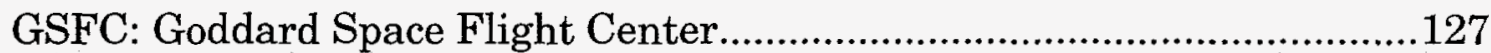

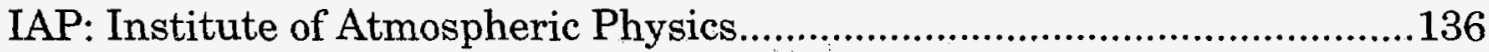

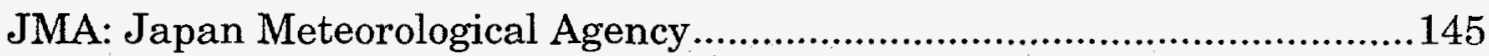

LMD: Laboratoire de Météorologie Dynamique...........................................153

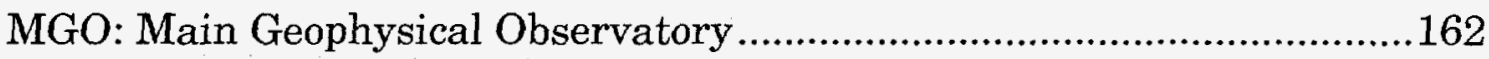

MPI: Max-Planck-Institut fuer Meteorologie ...........................................170

MRI: Meteorological Research Institute................................................180

NCAR: National Center for Atmospheric Research....................................190

NMC: National Meteorological Center ...................................................201

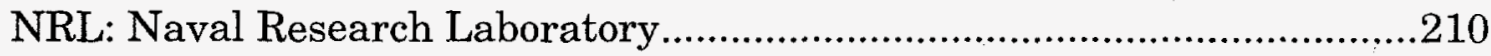

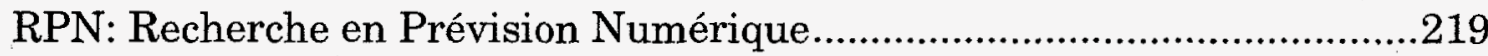

SUNYA: State University of New York at Albany .....................................227

SUNYA/NCAR: State University of New York at Albany/
National Center for Atmospheric Research ........................234

UCLA: University of California at Los Angeles ............................................245

UGAMP: The UK Universities' Global Atmospheric

UIUC: University of Illinois at Urbana-Champaign ..................................265

UKMO: United Kingdom Meteorological Office........................................274

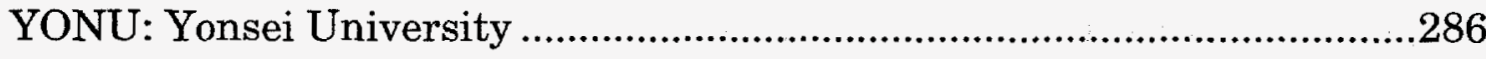




\begin{abstract}
This report summarizes essential features of the global atmospheric models of 30 groups that are participating in the Atmospheric Model Intercomparison Project (AMIP), an international effort to simulate the climate of the period 1979-1988 with common ocean boundary conditions and radiative forcings. Salient features of the AMIP models are summarized in tabular form, and the principal numerical, dynamical, and physical properties of each model are described in greater detail as well. An extensive bibliography of references on the algorithms and parametrizations used in the AMIP models is also provided.
\end{abstract}




\section{Acknowledgments}

The cooperation of the representatives of the AMIP modeling groups in the production of this report is gratefully acknowledged. In addition, Jay Cherniak, Elaine Price, and W. Lawrence Gates provided helpful editorial assistance, and Anna McCravy contributed substantial clerical support. This work was performed under the auspices of the U.S. Department of Energy, Environmental Sciences Division, by the Lawrence Livermore National Laboratory under Contract W-7405-ENG-48. 


\section{Acronyms Glossary}

ACSYS

AMIP

BMRC

CCC

CHAMMP

CLIMAP

CNRM

COLA

CSIRO

CSU

DERF

DKRZ

DNM

DOE

ECMWF

FGGE

FNOC

GARP

GCIP

GEWEX

GFDL

GISS

GLA

GSFC

IAP

ICSU

JMA

LLNL
Arctic Climate System Study

Atmospheric Model Intercomparison Project

Bureau of Meteorology Research Centre

Canadian Centre for Climate Research

Computer Hardware, Advanced Mathematics and Model Physics

Climate, Long-Range Investigation, Mapping and Prediction

Centre National de Recherches Météorologiques

Center for Ocean-Land-Atmosphere Studies

Commonwealth Scientific and Industrial Research Organization

Colorado State University

Dynamical Extended-Range Forecasting

Deutsches Klimarechenzentrum

Department of Numerical Mathematics

Department of Energy

European Centre for Medium-Range Weather Forecasts

First GARP Global Experiment

Fleet Naval Oceanographic Center

Global Atmospheric Research Program

GEWEX Continental-Scale International Project

Global Energy and Water Cycle Experiment

Geophysical Fluid Dynamics Laboratory

Goddard Institute for Space Studies

Goddard Laboratory for Atmospheres

Goddard Space Flight Center

Institute of Atmospheric Physics

International Council of Scientific Unions

Japan Meteorological Agency

Lawrence Livermore National Laboratory 


\section{Acronyms Glossary (Continued)}

LMD

MGO

MONEG

MPI

MRI

NASA

NCAR

NERSC

NMC

NOAA

NRL

PCMDI

PILPS

RPN

SIOMP

SUNYA

SUNYANCAR

TOGA

UCLA

UGAMP

UIUC

UKMO

WCRP

WGNE

WMO

YONU
Laboratoire de Météorologie Dynamique

Main Geophysical Observatory

Monsoon Numerical Experimentation Group

Max-Planck-Institut fuer Meteorologie

Meteorological Research Institute

National Aeronautics and Space Administration

National Center for Atmospheric Research

National Energy Research Supercomputer Center

National Meteorological Center

National Oceanic and Atmospheric Administration

Naval Research Laboratory

Program for Climate Model Diagnosis and Intercomparison

Project for Intercomparison of Land-Surface Parameterization

Schemes

Recherche en Prévision Numérique

Sea Ice and Ocean Modelling Panel

State University of New York at Albany

State University of New York at Albany/

National Center for Atmospheric Research

Tropical Ocean-Global Atmosphere

University of California at Los Angeles

The UK Universities' Global Atmospheric Modelling Programme

University of Illinois at Urbana-Champaign

United Kingdom Meteorological Office

World Climate Research Programme

Working Group on Numerical Experimentation

World Meteorological Organization

Yonsei University 


\section{Introduction}

The Atmospheric Model Intercomparison Project (AMIP) is an international effort to simulate the global climate of the period 1979-1988 using certain common boundary conditions and radiative forcings (cf. Gates 1992). Scientific direction for the AMIP is provided by the Working Group on Numerical Experimentation (WGNE) on behalf of the World Climate Research Programme (WCRP). Detailed coordination of this intercomparison project is the responsibility of the Program for Climate Model Diagnosis and Intercomparison (PCMDI), which is funded by the U.S. Department of Energy (DOE). The AMIP is also coordinated with the DOE Computer Hardware, Advanced Mathematics and Model Physics (CHAMMP) Program (cf. Bader et al. 1992). In addition, the DOE has provided substantial funding for the execution of AMIP computer simulations at the National Energy Research Supercomputer Center (NERSC) of the Lawrence Livermore National Laboratory (LLNL), making possible the involvement of many modeling groups that would otherwise lack the necessary resources. To date, some 30 groups representing most of the world's global modeling centers are participating in the AMIP (see Table 1-1).

The boundary conditions common to all the AMIP simulations consist of 120 monthly observed distributions of sea surface temperatures and sea ice extents obtained using available in-situ, climatological, and satellite data. These data were prepared by the Climate Analysis Center of the National Oceanic and Atmospheric Administration (NOAA) in cooperation with the Center for Ocean-Land-Atmosphere Interactions at the University of Maryland. (The PCMDI subsequently disseminated an edited version of these data to each AMIP modeling group.) There are also identical specifications of the solar constant $\left(1365 \mathrm{~W} \mathrm{~m}^{-2}\right)$ and atmospheric carbon dioxide concentration (a uniform $345 \mathrm{ppm}$ ) in the AMIP simulations.

In addition, the AMIP effort involves the generation of common monthly mean diagnostics in a standard format, and validation against observational data for the period 1979 to 1988. Diagnosis and validation of the AMIP model simulations is the special responsibility of some 25 WGNE-approved diagnostic subprojects, which are focussed on the analysis of the simulations of particular physical and dynamical processes or of climatic features of particular regions. Some of these investigations are being coordinated with other WCRP climate initiatives (see Table 1-2). 
Table 1-1: AMIP modeling groups. List of the AMIP modeling groups and their locations.

\begin{tabular}{|c|c|c|}
\hline Acronym & AMIP Group & Location \\
\hline BMRC & Bureau of Meteorology Research Centre & Melbourne, Australia \\
\hline $\mathrm{CCC}$ & Canadian Centre for Climate Research & Victoria, Canada \\
\hline CNRM & Centre National de Recherches Météorologiques & Toulouse, France \\
\hline COLA & Center for Ocean-Land-Atmosphere Studies & Calverton, Maryland (USA) \\
\hline CSIRO & Commonwealth Scientific \& Industrial Research Organization & Mordialloc, Australia \\
\hline$\overline{\mathbf{C S U}}$ & Colorado State University & Fort Collins, Colorado (USA) \\
\hline DERF & Dynamical Extended Range Forecasting (at GFDL) & Princeton, New Jersey (USA) \\
\hline DNM & $\begin{array}{l}\text { Department of Numerical Mathematics } \\
\text { (of the Russian Academy of Sciences) }\end{array}$ & Moscow, Russia \\
\hline ECMWF & European Centre for Medium-Range Weather Forecasts & Reading, England \\
\hline GFDL & Geophysical Fluid Dynamics Laboratory & Princeton, New Jersey (USA) \\
\hline GISS & Goddard Institute for Space Studies & New York, New York (USA) \\
\hline GLA & Goddard Laboratory for Atmospheres & Greenbelt, Maryland (USA) \\
\hline GSFC & Goddard Space Flight Center & Greenbelt, Maryland (USA) \\
\hline IAP & $\begin{array}{l}\text { Institute of Atmospheric Physics } \\
\text { (of the Chinese Academy of Sciences) }\end{array}$ & Beijing, China \\
\hline JMA & Japan Meteorological Agency & Tokyo, Japan \\
\hline LMD & Laboratoire de Météorologie Dynamique & Paris, France \\
\hline MGO & Main Geophysical Observatory & St. Petersburg, Russia \\
\hline MPI & Max Planck Institut fuer Meteorologie & Hamburg, Germany \\
\hline MRI & Meteorological Research Institute & Ibaraki-ken, Japan \\
\hline NCAR & National Center for Atmospheric Research & Boulder, Colorado (USA) \\
\hline NMC & National Meteorological Center & Suitland, Maryland (USA) \\
\hline NRL & Naval Research Laboratory & Monterey, California (USA) \\
\hline $\mathbf{R P N}$ & Recherche en Prévision Numérique & Dorval, Canada \\
\hline SUNYA & State University of New York at Albany & Albany, New York (USA) \\
\hline SUNYANCAR & $\begin{array}{l}\text { State University of New York at Albany/ } \\
\text { National Center for Atmospheric Research }\end{array}$ & $\begin{array}{l}\text { Albany, New York } \\
\text { Boulder, Colorado (USA) }\end{array}$ \\
\hline UCLA & University of California at Los Angeles & Los Angeles, California (USA) \\
\hline UGAMP & $\begin{array}{l}\text { The UK Universities' Global Atmospheric Modelling } \\
\text { Programme }\end{array}$ & Reading, England \\
\hline UIUC & University of Illinois at Urbana-Champaign & Urbana, Illinois (USA) \\
\hline UKMO & United Kingdom Meteorological Office & Bracknell, United Kingdom \\
\hline YONU & Yonsei University & Seoul, Korea \\
\hline
\end{tabular}


Table 1-2: AMIP diagnostic subprojects. List of the AMIP diagnostic subprojects and their scientific foci. (Coordination with other World Climate Research Programme initiatives is noted as appropriate.)

\begin{tabular}{|c|c|}
\hline Subproject & Scientific Focus \\
\hline 1 & Variability of the tropics: synoptic to intraseasonal time scales \\
\hline 2 & Intercomparison of low frequency variability \\
\hline 3 & Cyclone frequencies and extratropical intraseasonal variability \\
\hline 4 & Clear-sky greenhouse sensitivity, water vapor distribution, and cloud radiative forcing \\
\hline 5 & Surface boundary fluxes over the oceans \\
\hline 6 & Monsoons (coordinated with MONEG/TOGA,WGNE) \\
\hline 7 & Intercomparison of hydrologic processes in general circulation models \\
\hline 8 & Polar phenomena and sea ice (coordinated with SIOMP/ACSYS) \\
\hline 9 & Validation of high latitude tropospheric circulation in the Southern Hemisphere \\
\hline 10 & Diagnostics of atmospheric blocking in general circulation models \\
\hline 11 & Validation of humidity, moisture fluxes, and soil moisture in general circulation models \\
\hline 12 & Land surface processes \& parameterizations (coordinated with PILPS/GCIP/GEWEX,WGNE) \\
\hline 13 & Diagnoses of global cloudiness variations in model results and observational data \\
\hline 14 & Cloud radiative forcing: intercomparison and validation \\
\hline 15 & Atmospheric angular momentum fluctuations in global numerical models \\
\hline 16 & Simulations of the stratospheric circulation \\
\hline 17 & Multi-scale water and energy balance processes (coordinated with GCIP/GEWEX) \\
\hline 18 & Capability of current models to simulate extreme events and associated circulation patterns \\
\hline 19 & Model validation by microwave sounding unit (MSU) data \\
\hline 20 & Intercomparison of model simulated circulation features related to Southern Africa \\
\hline 21 & Surface monthly and daily time-scale climatologies and regional climate anomalies \\
\hline 22 & Comparative energetics analysis of climate models in the wavenumber domain \\
\hline 23 & Variations of the centers of action \\
\hline 24 & Analysis of Caspian Sea regional climate data as compared to AMIP model outputs \\
\hline $\mathbf{2 5}$ & General circulation model simulation of the East Asian climate \\
\hline
\end{tabular}

ACSYS: Arctic Climate System Study

GCIP: GEWEX Continental-Scale International Project

GEWEX: Global Energy and Water Cycle Experiment

MONEG: Monsoon Numerical Experimentation Group

PILPS:Project for Intercomparison of Land-Surface Parameterization Schemes

SIOMP: Sea Ice and Ocean Modelling Panel

TOGA: Tropical Ocean-Global Atmosphere

WGNE: Working Group on Numerical Experimentation 
A common goal of the diagnostic subprojects is to assess the phenomenological effects of applying diverse numerical schemes and physical parameterizations that are represented in the collection of AMIP models. Given the nonlinear behavior of global atmospheric models, the attribution of particular details of the AMIP simulations to these properties is very difficult and requires, as a minimum prerequisite, an accurate and comprehensive description of the numerics, dynamics, and physics of the models.

The present summary documentation of the AMIP models is written principally to serve this need of the diagnostic subprojects. This document centralizes information on the main features of the AMIP models and expresses this according to a common framework. This report is not intended, however, to substitute for existing documentation of these models, which is liberally cited throughout.

The sections of this report are organized in the order of progressive complexity of information content. That is, an overview of the major differences in the AMIP models' features may first be obtained by perusing the tables in Section 2, and further information may then be gleaned from the respective model feature descriptions in Section 3, which makes up the bulk of the report. Additional details on model algorithms and parameterizations can be obtained by consulting references in the extensive bibliography that follows Section 3 . 


\section{Summary Tables}

In this section, salient features of the AMIP models are displayed in a series of tables, each of which illustrates how a selected feature, or group of similar features, plays out across the models. It should be noted that these tables tend to highlight inter-model differences; the broad similarities among the AMIP models are better conveyed by the more detailed descriptions that make up Section 3 . The reader is also advised to consult Section 3 for information on the many nuances of the models' features that cannot be easily expressed in tables.

\section{List of Summary Tables}

Table 2-1: Model representation/resolution.

Table 2-2: Computational information.

Table 2-3: Initialization.

Table 2-4: Time integration.

Table 2-5: Filtering, smoothing, and filling.

Table 2-6: Diffusion and gravity-wave drag.

Table 2-7: Atmospheric chemistry.

Table 2-8: Atmospheric radiation.

Table 2-9: Cloud-radiative interactions.

Table 2-10: Convection.

Table 2-11: Cloud formation and precipitation.

Table 2-12: Planetary boundary layer.

Table 2-13: Snow cover and sea ice.

Table 2-14: Surface characteristics.

Table 2-15: Land surface processes. 
Table 2-1: Model representation/resolution. The table lists the horizontal representation and resolution; vertical coordinates and number of prognostic vertical levels (number below $800 \mathrm{hPa}$, above $200 \mathrm{hPa}$ ); and atmospheric bottom and top pressure levels, for a surface pressure of $1000 \mathrm{hPa}$.

\begin{tabular}{|c|c|c|c|c|c|}
\hline \multirow{2}{*}{ AMIP Model } & \multicolumn{2}{|c|}{ Horizontal } & \multicolumn{3}{|c|}{ Vertical } \\
\hline & Representation & Resolution & Coordinates & No. Levels & Bottom, Top \\
\hline BMRC & spectral & rhomboidal 31 & sigma & $9(3,3)$ & $991,9 \mathrm{hPa}$ \\
\hline$\overline{C C C}$ & spectral & triangular 32 & hybrid & $10(3,4)$ & $980,5 \mathrm{hPa}$ \\
\hline CNRM & spectral & triangular 42 & hybrid & $30(4,20)$ & $995,0.01 \mathrm{hPa}$ \\
\hline COLA & spectral & rhomboidal 40 & sigma & $18(5,4)$ & $995,10 \mathrm{hPa}$ \\
\hline CSIRO & spectral & rhomboidal 21 & sigma & $9(3,3)$ & $979,21 \mathrm{hPa}$ \\
\hline$\overline{\mathrm{CSU}}$ & finite difference & $4 \times 5$ degrees & modified sigma & $17(2,6)$ & variable, $51 \mathrm{hPa}$ \\
\hline DERF & spectral & triangular 42 & sigma & $18(5,5)$ & $998,2 \mathrm{hPa}$ \\
\hline DNM & finite difference & $4 \times 5$ degrees & sigma & $7(1,1)$ & $929,71 \mathrm{hPa}$ \\
\hline ECMWF & spectral & triangular 42 & hybrid & $19(5,7)$ & $996,10 \mathrm{hPa}$ \\
\hline GFDL & spectral & rhomboidal 30 & sigma & $14(4,4)$ & $997,15 \mathrm{hPa}$ \\
\hline GISS & finite difference & $4 \times 5$ degrees & sigma & $9(2,2)$ & $975,10 \mathrm{hPa}$ \\
\hline GLA & finite difference & $4 \times 5$ degrees & sigma & $17(5,4)$ & $994,12 \mathrm{hPa}$ \\
\hline$\overline{\text { GSFC }}$ & finite difference & $4 \times 5$ degrees & sigma & $20(5,7)$ & $994,10 \mathrm{hPa}$ \\
\hline IAP & finite difference & $4 \times 5$ degrees & modified sigma & $2(0,0)$ & $800,200 \mathrm{hPa}$ \\
\hline JMA & spectral & triangular 42 & hybrid & $21(6,7)$ & $995,10 \mathrm{hPa}$ \\
\hline LMD & finite difference & 50 sinlat $\times 64$ lon & sigma & $11(3,2)$ & $979,4 \mathrm{hPa}$ \\
\hline MGO & spectral & triangular 30 & sigma & $14(5,4)$ & $992,13 \mathrm{hPa}$ \\
\hline MPI & spectral & triangular 42 & hybrid & $19(5,7)$ & $996,10 \mathrm{hPa}$ \\
\hline MRI & finite difference & $4 \times 5$ degrees & hybrid & $15(1,9)$ & variable, $1 \mathrm{hPa}$ \\
\hline NCAR & spectral & triangular 42 & hybrid & $18(4,7)$ & $992,3 \mathrm{hPa}$ \\
\hline NMC & spectral & triangular 40 & sigma & $18(5,4)$ & $995,21 \mathrm{hPa}$ \\
\hline NRL & spectral & triangular 47 & hybrid & $18(5,5)$ & $995,1 \mathrm{hPa}$ \\
\hline $\mathbf{R P N}$ & $\begin{array}{l}\text { spectral semi- } \\
\text { Lagrangian }\end{array}$ & triangular 63 & sigma & $23(7,7)$ & $1000,10 \mathrm{hPa}$ \\
\hline SUNYA & spectral & rhomboidal 15 & sigma & $12(3,5)$ & $991,9 \mathrm{hPa}$ \\
\hline SUNYA/NCAR & spectral & triangular 31 & hybrid/sigma & $18(4,7)$ & $993,5 \mathrm{hPa}$ \\
\hline UCLA & spectral & $4 \times 5$ degrees & modified sigma & $15(2,9)$ & variable, $1 \mathrm{hPa}$ \\
\hline UGAMP & spectral & triangular 42 & hybrid & $19(5,7)$ & $996,10 \mathrm{hPa}$ \\
\hline UחUC & finite difference & $4 \times 5$ degrees & sigma & $7(3,0)$ & $990,200 \mathrm{hPa}$ \\
\hline UKMO & finite difference & $2.5 \times 3.75$ degrees & hybrid & $19(4,7)$ & $997,5 \mathrm{hPa}$ \\
\hline YONU & finite difference & $4 \times 5$ degrees & modified sigma & $5(1,1)$ & $900,100 \mathrm{hPa}$ \\
\hline
\end{tabular}


Table 2-2: Computational information. The table lists the computer, number of processors, and operating system utilized; the computational performance (in minutes of computing time per simulated day); and the history storage interval.

\begin{tabular}{|c|c|c|c|c|}
\hline AMIP Model & Computer (No. Processors) & Environment & Performance & Storage \\
\hline BMRC & Cray Y/MP (1) & UNICOS & 3 minutes/day & 6 hours \\
\hline$\overline{\mathbf{C C C}}$ & Cray X/MP (1) & $\cos 1.17$ & 6 minutes/day & 6 hours \\
\hline CNRM & Cray $2(1)$ & UNICOS & 15 minutes/day & 6 hours \\
\hline COLA & Cray 2(1) & UNICOS & 18 minutes/day & 6 hours \\
\hline CSIRO & Cray Y/MP (1) & UNICOS & 0.6 minutes/day & 6 hours \\
\hline CSU & Cray $2(1)$ & CTSS & 6.5 minutes/day & 6 hours \\
\hline DERF & Cray Y/MP (1) & UNICOS & 5.5 minutes/day & 6 hours \\
\hline DNM & Cray $2(1)$ & UNICOS & 3 minutes/day & 6 hours \\
\hline ECMWF & Cray $2(1)$ & UNICOS & 15 minutes/day & 6 hours \\
\hline GFDL & Cray Y/MP (1) & UNICOS & 1 minute/day & 24 hours \\
\hline GISS & IBM PowerStation 580 (multiple) & UNIX & 10 minutes/day & 24 hours \\
\hline GLA & Cray Y/MP (1) & UNICOS & 6 minutes/day & 6 hours \\
\hline GSFC & Cray Y/MP (1) & UNICOS & 4 minutes/day & 6 hours \\
\hline$\overline{I A P}$ & Convex-C120 (1) & Convex & 5 minutes/day & 6 hours \\
\hline JMA & HITAC S-810 (1) & VOS3/HAP/ES & 2 minutes/day & 6 hours \\
\hline LMD & Cray 2(1) & UNICOS & 2 minutes/day & 24 hours \\
\hline MGO & Cray $2(1)$ & UNICOS & 6 minutes/day & 24 hours \\
\hline MPI & Cray $2(1)$ & UNICOS & 8 minutes/day & 6 hours \\
\hline MRI & HITAC S-810/10 (1) & VOS3 & 6 minutes/day & 6 hours \\
\hline NCAR & Cray 2 (multiple) & UNICOS & 7 minutes/day & 6 hours \\
\hline NMC & Cray Y/MP (8) & UNICOS & 4 minutes/day & 6 hours \\
\hline NRL & Cray Y/MP (4) & UNICOS & 10 minutes/day & 6 hours \\
\hline RPN & NEC SX-3 (1) & UNIX & 4 minutes/day & 12 hours \\
\hline SUNYA & Cray 2(1) & UNICOS & 1.2 minutes/day & 12 hours \\
\hline SUNYA/NCAR & Cray Y/MP (1) & UNICOS & 4 minutes/day & 24 hours \\
\hline UCLA & Cray C90 (1) & UNICOS & 1.5 minutes/day & 6 hours \\
\hline UGAMP & Cray 2(1) & UNICOS & 8 minutes/day & 6 hours \\
\hline UIUC & Cray C90 (1) & UNICOS & 1.3 minutes/day & 6 hours \\
\hline UKMO & Cray Y/MP (2) & UNICOS & 4.7 minutes/day & 6 hours \\
\hline YONU & Cray C90 (1) & UNICOS & 3 minutes/day & 6 hours \\
\hline
\end{tabular}


Table 2-3: Initialization. The table lists the data used (observational analyses, climatological datasets, or previous model solutions) for initialization of the atmosphere, soil moisture, and snow cover/depth.

\begin{tabular}{|c|c|c|c|}
\hline \multirow{2}{*}{ AMLP Model } & \multicolumn{3}{|c|}{ Initialization Data } \\
\hline & Atmosphere & Soil Moisture & Snow Cover/Depth \\
\hline BMRC & ECMWF III-B analysis & Mintz and Serafini (1989) & Hummel and Reck (1979) \\
\hline $\mathrm{CCC}$ & FGGE III-B analysis & previous model solution & previous model solution \\
\hline CNRM & previous model solution & previous model solution & previous model solution \\
\hline COLA & NMC analysis & GFDL climatology & GFDL climatology \\
\hline CSIRO & previous model solution & previous model solution & previous model solution \\
\hline CSU & previous model solution & Mintz and Serafini (1989) & previous model solution \\
\hline DERF & NMC analysis & ECMWF analysis & ECMWF analysis \\
\hline DNM & previous model solution & previous model solution & previous model solution \\
\hline ECMWF & ECMWF analysis & ECMWF analysis & ECMWF analysis \\
\hline GFDL & previous model solution & previous model solution & previous model solution \\
\hline GISS & previous model solution & previous model solution & previous model solution \\
\hline GLA & ECMWF analysis & previous model solution & previous model solution \\
\hline GSFC & ECMWF FGGE reanalysis & Schemm et al. (1992) & NASA climatology \\
\hline IAP & previous model solution & previous model solution & previous model solution \\
\hline JMA & ECMWF FGGE III-B analysis & Willmott et al. (1985) & Dewey (1987) \\
\hline $\mathbf{L M D}$ & previous model solution & previous model solution & previous model solution \\
\hline MGO & ECMWF analysis & Mintz and Serafini (1989) & ECMWF climatology \\
\hline MPI & ECMWF analysis & ECMWF climatology & ECMWF climatology \\
\hline MRI & previous model solution & previous model solution & previous model solution \\
\hline NCAR & previous model solution & prescribed surface wetness & Forderhase et al. (1980) \\
\hline NMC & NMC analysis & NMC "Launcher" climatology & NMC "Launcher" climatology \\
\hline NRL & ECMWF FGGE III-B analysis & FNOC climatology & initially zero everywhere \\
\hline RPN & FGGE analysis & FGGE climatology & FGGE climatology \\
\hline SUNYA & previous model solution & previous model solution & previous model solution \\
\hline SUNYA/NCAR & previous model solution & previous model solution & previous model solution \\
\hline UCLA & FGGE III-B analysis & Mintz and Serafini (1981) & FGGE III-B analysis \\
\hline UGAMP & ECMWF analysis & ECMWF analysis & ECMWF analysis \\
\hline UIUC & previous model solution & previous model solution & previous model solution \\
\hline UKIMO & previous model solution & previous model solution & previous model solution \\
\hline YONU & previous model solution & previous model solution & previous model solution \\
\hline
\end{tabular}


Table 2-4: Time integration. The table lists the numerical schemes and time-step lengths for integration of dynamics and full shortwave and longwave radiation calculations (with recalculation intervals of longwave absorptivities/emissivities, where different, noted in parentheses).

\begin{tabular}{|c|c|c|c|c|}
\hline \multirow{3}{*}{ AMIP Model } & \multicolumn{4}{|c|}{ Time Integration } \\
\hline & \multirow{2}{*}{ Schemes } & \multicolumn{3}{|c|}{ Time-Step Lengths } \\
\hline & & Dynamics & Shortwave & Longwave \\
\hline BMRC & semi-implicit, split implicit & 15 minutes & 3 hours & 3 hours \\
\hline $\mathbf{C C C}$ & semi-implicit & 20 minutes & 3 hours & 6 hours \\
\hline CNRM & semi-implicit & 15 minutes & 15 minutes & 15 minutes \\
\hline COLA & semi-implicit, implicit & 12 minutes & 1 hour & 3 hours \\
\hline$\overline{\text { CSIRO }}$ & semi-implicit, split backward implicit & 30 minutes & 2 hours & 2 hours \\
\hline CSU & leapfrog, Matsuno, implicit, forward & 6 minutes & 1 hour & 1 hour \\
\hline DERF & semi-implicit & 15 minutes & 12 hours & 12 hours \\
\hline DNM & Matsuno & 6 minutes & 3 hours & 3 hours \\
\hline ECMWF & semi-implicit & 30 minutes & 3 hours & 3 hours \\
\hline GFDL & semi-implicit & 18 minutes & 24 hours & 24 hours \\
\hline GISS & leapfrog, Euler-backward & 7.5 minutes & 5 hours & 5 hours \\
\hline GLA & leapfrog, Matsuno, backward implicit, forward & 3.75 minutes & 30 minutes & 1 hour \\
\hline GSFC & leapfrog, backward implicit & 5 minutes & 3 hours & 3 hours \\
\hline IAP & leapfrog & 6 minutes & 1 hour & 1 hour \\
\hline JMA & semi-implicit & variable & 1 hour & 3 hours \\
\hline LMD & leapfrog, Matsuno & 6 minutes & 6 hours & 6 hours \\
\hline MGO & two-step semi-implicit, split Euler-backward & 30 minutes & 12 hours & 12 hours \\
\hline MPI & semi-implicit & 24 minutes & 2 hours & 2 hours \\
\hline MRI & leapfrog, Matsuno, backward implicit & 6 minutes & 1 hour & $1 \mathrm{hr}(3 \mathrm{hrs})$ \\
\hline NCAR & semi-implicit, split implicit & 20 minutes & 1 hour & $1 \mathrm{hr}(12 \mathrm{hrs})$ \\
\hline NMC & semi-implicit & 30 minutes & 3 hours & 3 hours \\
\hline NRL & semi-implicit, implicit & 20 minutes & 1.5 hours & 1.5 hours \\
\hline RPN & semi-implicit semi-Lagrangian, implicit & 30 minutes & 3 hours & 3 hours \\
\hline SUNYA & semi-implicit & 30 minutes & 12 hours & $0.5 \mathrm{hr}(24 \mathrm{hrs})$ \\
\hline SUNYA/NCAR & semi-implicit & 30 minutes & 1.5 hours & $0.5 \mathrm{hr}(24 \mathrm{hrs})$ \\
\hline UCLA & leapfrog, Matsuno & 7.5 minutes & 1 hour & 1 hour \\
\hline UGAMP & semi-implicit & 30 minutes & $\begin{array}{l}3 \text { hours (on a } \\
\text { reduced grid) }\end{array}$ & $\begin{array}{l}3 \text { hours (on a } \\
\text { reduced grid) }\end{array}$ \\
\hline UIUC & leapfrog, Matsuno & 6 minutes & 1 hour & 1 hour \\
\hline UKMO & $\begin{array}{l}\text { split forward-backward and two-step Heun, } \\
\text { implicit }\end{array}$ & 30 minutes & 3 hours & 3 hours \\
\hline YONU & leapfrog, Matsuno & 7.5 minutes & 1 hour & 1 hour \\
\hline
\end{tabular}


Table 2-5: Filtering, smoothing, and filling. The table lists the instances of application of time filtering, spatial filtering/smoothing of model atmosphere and orography, or filling of spurious negative values of atmospheric moisture.

\begin{tabular}{|c|c|c|c|c|}
\hline \multirow{2}{*}{ AMIP Model } & \multirow{2}{*}{ Time Filtering } & \multicolumn{2}{|c|}{ Spatial Filtering/Smoothing } & \multirow{2}{*}{ Moisture Filling } \\
\hline & & Atmosphere & Orography & \\
\hline BMRC & $\mathrm{x}$ & & $\mathrm{x}$ & $\mathrm{x}$ \\
\hline$\overline{\mathbf{C C C}}$ & $\mathrm{x}$ & & & $\mathrm{x}$ \\
\hline CNRM & $\mathrm{x}$ & & & $\bar{x}$ \\
\hline$\overline{\text { COLA }}$ & $\bar{x}$ & & & $\bar{x}$ \\
\hline CSIRO & $\bar{x}$ & & & $\bar{x}$ \\
\hline CSU & & $\overline{\mathrm{x}}$ & $\mathrm{x}$ & $\bar{x}$ \\
\hline DERF & $\bar{x}$ & & & $\mathrm{x}$ \\
\hline DNM & & $\bar{x}$ & $\mathrm{x}$ & $\mathrm{x}$ \\
\hline ECMWF & $\mathrm{x}$ & & $\mathrm{x}$ & $\mathrm{x}$ \\
\hline GFDL & $\mathrm{x}$ & & $\mathrm{x}$ & $\bar{x}$ \\
\hline GISS & & $\mathrm{x}$ & & $\mathrm{x}$ \\
\hline GLA & & $\mathbf{x}$ & $\mathrm{x}$ & $\mathrm{x}$ \\
\hline GSFC & $\mathrm{x}$ & $\bar{x}$ & $\bar{x}$ & $\mathrm{x}$ \\
\hline IAP & $\mathrm{x}$ & $\mathrm{x}$ & $\mathrm{x}$ & $\mathrm{x}$ \\
\hline JMA & $\mathrm{x}$ & & & $\bar{x}$ \\
\hline LMD & & $\bar{x}$ & & $\bar{x}$ \\
\hline MGO & & & $\mathrm{x}$ & $\mathrm{x}$ \\
\hline MPI & $\mathrm{x}$ & & $x$ & for radiation only \\
\hline MRI & & $\mathrm{x}$ & $\mathrm{x}$ & \\
\hline NCAR & $\mathrm{x}$ & & $\mathrm{x}$ & \\
\hline NMC & $\mathrm{x}$ & & & \\
\hline NRL & $\mathrm{x}$ & & $x$ & $\mathbf{x}$ \\
\hline RPN & $\bar{x}$ & & $\mathrm{x}$ & \\
\hline SUNYA & $\mathrm{x}$ & & $\mathrm{x}$ & $\bar{x}$ \\
\hline SUNYANNCAR & $x$ & & & \\
\hline$\overline{\text { UCLA }}$ & & $\mathrm{x}$ & & \\
\hline UGAMP & $\mathrm{x}$ & & $\mathrm{x}$ & $\bar{x}$ \\
\hline UTUC & & $\bar{x}$ & & \\
\hline UKMO & & $\bar{x}$ & $\mathbf{x}$ & $\mathrm{x}$ \\
\hline YONU & & $x$ & & \\
\hline
\end{tabular}


Table 2-6: Diffusion and gravity-wave drag. The table lists the type of horizontal diffusion, if present; whether there is vertical diffusion of momentum, heat, or moisture above the planetary boundary layer (PBL); and references on the gravity-wave drag scheme, if present.

\begin{tabular}{|c|c|c|c|}
\hline AMIP Model & Horizontal Diffusion & $\begin{array}{c}\text { Vert. Diffusion } \\
\text { Above PBL }\end{array}$ & Gravity-wave Drag Schemes \\
\hline BMRC & scale-selective $\nabla^{2}$ & $\begin{array}{l}\text { only to } \sigma=0.5 \text { in } \\
\text { stable conditions }\end{array}$ & Palmer et al. (1986) \\
\hline $\mathrm{CCC}$ & $\begin{array}{l}\text { scale-selective function of } \\
\text { wave number }\end{array}$ & $\mathrm{x}$ & McFarlane (1987) \\
\hline CNRM & linear $\nabla^{4}$ & up to $25 \mathrm{hPa}$ & Clary (1987) \\
\hline COLA & linear $\nabla^{4}$ & $\mathbf{x}$ & Kirtman et al. (1993), Alpert et al. (1988) \\
\hline CSIRO & scale-selective $\nabla^{2}$ & $\mathrm{x}$ & Chouinard et al. (1986) \\
\hline$\overline{\mathrm{CSU}}$ & nonlinear & & \\
\hline DERF & linear $\nabla^{4}$ & $\mathrm{x}$ & Stern and Pierrehumbert (1988) \\
\hline$\overline{D N M}$ & nonlinear & & \\
\hline ECMWF & linear $\nabla^{4}$ & $\begin{array}{l}\text { for unstable } \\
\text { stratification }\end{array}$ & Palmer et al. (1986), Miller et al. (1989) \\
\hline GFDL & linear $\nabla^{4}$ & up to about $5 \mathrm{~km}$ & Broccoli and Manabe (1992) \\
\hline GISS & & & Hansen et al. (1983) \\
\hline$\overline{\text { GLAA }}$ & & $\mathrm{x}$ & \\
\hline GSFC & & $\mathrm{x}$ & \\
\hline IAP & nonlinear & & \\
\hline JMA & linear $\nabla^{4}$ & $\mathrm{x}$ & Iwasaki et al. $(1989 a, b)$ \\
\hline LMD & linear $\nabla^{4}$ & & Boer et al. (1984a) \\
\hline MGO & scale-selective $\nabla^{2}$ & $\mathrm{x}$ & McFarlane (1987) \\
\hline MPI & scale-selective $\nabla^{4}$ & $\begin{array}{l}\text { for unstable } \\
\text { stratification }\end{array}$ & Palmer et al. (1986), Miller et al. (1989) \\
\hline MRI & nonlinear (momentum only) & & $\begin{array}{l}\text { Palmer et al. (1986), } \\
\text { Yagai \& Yamazaki (1988) }\end{array}$ \\
\hline NCAR & linear $\nabla^{4}$, scale-selective $\nabla^{2}$ & $\mathrm{x}$ & McFarlane (1987) \\
\hline NMC & scale-selective $\nabla^{2}$ & $\mathrm{x}$ & Alpert et al. (1988), Pierrehumbert (1987) \\
\hline NRL & linear $\nabla^{4}$ & $\mathrm{x}$ & modified Palmer et al. (1986) \\
\hline $\mathbf{R P N}$ & linear $\nabla^{2}$ & $\mathrm{x}$ & McFarlane (1987) \\
\hline SUNYA & linear $\nabla^{4}$ and $\nabla^{2}$ & $\mathrm{x}$ & \\
\hline SUNYANCAR & linear $\nabla^{4}$ and $\nabla^{2}$ & $\mathrm{x}$ & McFarlane (1987) \\
\hline UCLA & nonlinear & & Palmer et al. (1986) \\
\hline UGAMP & linear $\nabla^{6}$ & $\begin{array}{c}\text { cut-off above } \\
\text { hybrid level } 0.65\end{array}$ & Palmer et al. (1986) \\
\hline UIUC & & $\mathrm{x}$ & \\
\hline UKMO & linear $\nabla^{6}$ and $\nabla^{4}$ & & Wilson and Swinbank (1989) \\
\hline YONU & nonlinear (momentum only) & $\mathrm{x}$ & \\
\hline
\end{tabular}


Table 2-7: Atmospheric chemistry. The table lists the constituents (excluding uniformly mixed carbon dioxide at $345 \mathrm{ppm}$ concentration and prognostic water vapor) that are included in the atmospheric radiation calculations. For prescribed ozone concentrations, data references are listed; for prognostic ozone, the parameterization schemes are referenced.

\begin{tabular}{|c|c|c|c|}
\hline \multirow{2}{*}{ AMIP Model } & \multicolumn{3}{|c|}{ Chemical Constituents } \\
\hline & Ozone References & Other Gases (except $\mathrm{CO}_{2}, \mathrm{H}_{2} \mathrm{O}$ ) & Aerosol \\
\hline$\overline{\text { BMRC }}$ & Dopplick (1974) & & \\
\hline$\overline{\mathbf{C C C}}$ & Wilcox \& Belmont (1977) & & \\
\hline CNRM & $\begin{array}{l}\text { prognostic--cf. Cariolle \& Déqué (1986), } \\
\text { Cariolle et al. (1990) }\end{array}$ & $\begin{array}{l}\text { oxygen, methane, carbon monoxide, } \\
\text { nitrous oxide }\end{array}$ & $\mathrm{x}$ \\
\hline COLA & GFDL climatologies & & \\
\hline CSIRO & Dopplick (1974) & & \\
\hline CSU & McPeters et al. (1984) & & \\
\hline DERF & GFDL climatologies & & \\
\hline DNM & $\begin{array}{l}\text { Koprova \& Uranova (1978), } \\
\text { Lacis \& Hansen (1974) }\end{array}$ & & \\
\hline ECMWF & London et al. (1986), Wilcox \& Belmont (1977) & $\begin{array}{l}\text { oxygen, methane, carbon monoxide, } \\
\text { nitrous oxide }\end{array}$ & $\mathrm{x}$ \\
\hline GFDL & Hering \& Borden (1965) & & \\
\hline GISS & London et al. (1976) & $\begin{array}{l}\text { oxygen, methane, nitrous oxide, } \\
\text { nitric oxide }\end{array}$ & $\mathrm{x}$ \\
\hline$\overline{\text { GLA }}$ & Rosenfield et al. (1987) & & $\mathrm{x}$ \\
\hline GSFC & Rosenfield et al. (1987) & & \\
\hline IAP & Duetsch (1971) & & \\
\hline JMA & McPeters et al. (1984) & & \\
\hline LMD & prognostic--cf. Royer et al. (1988) & & \\
\hline MGO & McPeters et al. (1984) & $\begin{array}{l}\text { methane, nitrous oxide, } \\
\text { chlorofluorocarbons CFC-11 \& CFC-12 }\end{array}$ & \\
\hline MPI & London et al. (1976), Wilcox \& Belmont (1977) & & $\mathrm{x}$ \\
\hline MRI & McPeters et al. (1984) & & \\
\hline NCAR & Duetsch (1978) & oxygen & \\
\hline NMC & Hering \& Borden (1965), London (1962) & & \\
\hline NRL & Dopplick (1974) & & \\
\hline $\mathbf{R P N}$ & Kita \& Sumi (1976) & & \\
\hline SUNYA & Duetsch (1978) & $\begin{array}{l}\text { oxygen, methane, nitrous oxide, } \\
\text { chlorofluorocarbons CFC-11 \& CFC- } 12\end{array}$ & \\
\hline SUNYA/NCAR & Wang et al. (1994) & $\begin{array}{l}\text { oxygen, methane, nitrous oxide, } \\
\text { chlorofluorocarbons CFC-11 \& CFC-12 }\end{array}$ & $\mathrm{x}$ \\
\hline UCLA & prognostic--cf. Schlesinger \& Mintz (1979) & & \\
\hline UGAMP & London et al. (1986), Wilcox \& Belmont (1977) & $\begin{array}{l}\text { oxygen, methane, carbon monoxide, } \\
\text { nitrous oxide }\end{array}$ & $\mathrm{x}$ \\
\hline UIUC & Total Ozone Mapping Spectrometer data & $\begin{array}{l}\text { methane, nitrous oxide, } \\
\text { chlorofluorocarbons CFC-11 \& CFC-12 }\end{array}$ & \\
\hline UKMO & Keating et al. (1987), McPeters et al. (1984) & & \\
\hline YONU & Bowman (1988) & $\begin{array}{l}\text { methane, nitrous oxide, } \\
\text { chlorofluorocarbons CFC-11 \& CFC-12 }\end{array}$ & \\
\hline
\end{tabular}


Table 2-8: Atmospheric radiation. The table lists whether a diurnal cycle in solar forcing is simulated and the references for the principal shortwave and longwave atmospheric radiation schemes.

\begin{tabular}{|c|c|c|c|}
\hline AMIP Model & Diurnal & Shortwave Radiation References & s Longwave Radiation References \\
\hline BMRC & $\bar{x}$ & Lacis \& Hansen (1974) & Fels-Schwarzkopf $(1975,1991)$ \\
\hline $\mathrm{CCC}$ & $\mathrm{x}$ & Fouquart \& Bonnel (1980) & Morcrette (1990, 1991), Clough et al. (1980) \\
\hline CNRM & $\mathrm{x}$ & $\begin{array}{l}\text { Ritter \& Geleyn (1992), } \\
\text { Zdunkowski et al. }(1980,1982)\end{array}$ & $\begin{array}{l}\text { Ritter \& Geleyn (1992), } \\
\text { Zdunkowski et al. }(1980,1982)\end{array}$ \\
\hline COLA & $\mathrm{x}$ & Harshvardhan et al. (1987) & Harshvardhan et al. (1987) \\
\hline CSIRO & $\mathrm{x}$ & $\begin{array}{l}\text { Lacis \& Hansen (1974), } \\
\text { Sasamori et al. (1972) }\end{array}$ & Fels-Schwarzkopf $(1975,1981,1991)$ \\
\hline CSU & $\mathrm{x}$ & $\begin{array}{l}\text { Harshvardhan et al. }(1987,1989) \text {, } \\
\text { Davies }(1982)\end{array}$ & Harshvardhan et al. $(1987,1989)$ \\
\hline DERF & & Lacis \& Hansen (1974) & Fels-Schwarzkopf $(1975,1991)$ \\
\hline DNM & $\mathrm{x}$ & $\begin{array}{l}\text { Manabe \& Strickler (1964), } \\
\text { Lacis \& Hansen (1974) }\end{array}$ & $\begin{array}{l}\text { Feigelson (1984), } \\
\text { Podolskaya \& Rivin (1988) }\end{array}$ \\
\hline ECMWF & $\mathrm{x}$ & Morcrette $(1989,1990,1991)$ & Morcrette $(1989,1990,1991)$ \\
\hline GFDL & & Lacis \& Hansen (1974) & $\begin{array}{l}\text { Rodgers \& Walshaw (1966), } \\
\text { Stone \& Manabe (1968) }\end{array}$ \\
\hline GISS & $\mathrm{x}$ & Lacis \& Hansen (1974) & Lacis \& Hansen (1974) \\
\hline GLA & $\mathrm{x}$ & Harshvardhan et al. (1987) & Harshvardhan et al. (1987) \\
\hline GSFC & $\bar{x}$ & Harshvardhan et al. (1987) & Harshvardhan et al. (1987) \\
\hline IAP & $\bar{x}$ & Cess (1985), Cess et al. (1985) & Katayama (1972) \\
\hline JMA & $\mathrm{x}$ & Lacis \& Hansen (1974) & Sugi et al. (1989) \\
\hline LMD & & Fouquart \& Bonnel (1980) & Morcrette (1990, 1991), Clough et al. (1980) \\
\hline MGO & & Karol (1986), Rozanov \& Frolkis (1988) & Karol (1986), Roberts et al. (1986) \\
\hline MPI & $\mathbf{x}$ & Hense et al. (1982) & Eickerling (1989) \\
\hline MRI & $\mathrm{x}$ & Lacis \& Hansen (1974) & Shibata \& Aoki (1989), Clough et al. (1980) \\
\hline NCAR & $\mathrm{x}$ & Briegleb (1992) & Kiehl \& Briegleb (1991) \\
\hline NMC & $\mathrm{x}$ & $\begin{array}{l}\text { Lacis \& Hansen (1974), } \\
\text { Sasamori et al. (1972) }\end{array}$ & Fels-Schwarzkopf $(1975,1991)$ \\
\hline NRL & $\mathbf{x}$ & Davies (1982), Lacis \& Hansen (1974) & Harshvardhan et al. (1987) \\
\hline $\mathbf{R P N}$ & $\mathrm{x}$ & Fouquart \& Bonnel (1980) & Garand (1983), Garand \& Mailhot (1990) \\
\hline SUNYA & & Kiehl et al. (1987) & Wang et al. $(1991 \mathrm{a}, \mathrm{b})$ \\
\hline SUNYA/NCAR & $\mathrm{x}$ & Kiehl et al. (1987) & Wang et al. (1991a, b) \\
\hline UCLA & $\mathrm{x}$ & Katayama (1972), Schlesinger (1976) & Harshvardhan et al. (1987) \\
\hline UGAMP & $\bar{x}$ & Morcrette $(1989,1990,1991)$ & Morcrette $(1989,1990,1991)$ \\
\hline UIUC & $\bar{x}$ & Oh (1989), Oh \& Schlesinger (1991c) & Oh (1989), Oh \& Schlesinger (1991c) \\
\hline UKMO & $\mathbf{x}$ & Ingram (1993) & Slingo \& Wilderspin (1986) \\
\hline YONU & $\mathbf{x}$ & Oh (1989), Oh \& Jung (1993) & $\mathrm{Oh}(1989)$ \\
\hline
\end{tabular}


Table 2-9: Cloud-radiative interactions. The table lists the treatment of shortwave and longwave cloudradiative interactions, whether these depend on (prognostic, diagnostic, or prescribed) cloud liquid water (CLW), and the assumption of cloud vertical overlap used in the radiation calculations (random, full, or mixed--both random and full, depending on cloud type or other factors).

\begin{tabular}{|c|c|c|c|c|}
\hline AMIP Model & Shortwave Interactions & Longwave Interactions & $\begin{array}{c}\text { Function } \\
\text { of CLW }\end{array}$ & $\begin{array}{l}\text { Vertical } \\
\text { Overlap }\end{array}$ \\
\hline BMRC & optics prescribed by cloud height & all clouds are blackbody emitters & & random \\
\hline$\overline{\mathrm{CCC}}$ & delta-Eddington & emissivity depends on optical depth & $\bar{x}$ & mixed \\
\hline CNRM & delta-two-stream & delta-two-stream & $\mathrm{x}$ & random \\
\hline COLA & delta-Eddington & emissivity depends on optical depth & $\mathrm{x}$ & mixed \\
\hline CSIRO & optics prescribed by cloud height & all clouds are blackbody emitters & & random \\
\hline$\overline{\mathrm{CSU}}$ & delta-Eddington & emissivity depends on optical depth & $\mathrm{x}$ & full \\
\hline DERF & delta-Eddington & emissivity depends on optical depth & $\mathrm{x}$ & random \\
\hline DNM & optics prescribed & emissivity < 1 for high cloud only & & random \\
\hline ECMWF & delta-Eddington & emissivity depends on CLW & $\mathrm{x}$ & mixed \\
\hline GFDL & optics depend on cloud height/depth & emissivity $<1$ for high cloud only & & full \\
\hline GISS & application of Mie theory & self-consistent with Mie theory & $\mathrm{x}$ & full \\
\hline GLA & delta-Eddington & emissivity depends on optical depth & & random \\
\hline$\overline{\text { GSFC }}$ & delta-Eddington & all clouds are blackbody emitters & & mixed \\
\hline IAP & delta-Eddington & emissivity $<1$ for cirrus cloud only & & mixed \\
\hline JMA & delta-Eddington & emissivity $<1$ for cirrus cloud only & & random \\
\hline LMD & delta-Eddington and two-stream & emissivity depends on CLW & $\mathrm{x}$ & random \\
\hline MGO & optics prescribed & emissivity depends on temperature & & mixed \\
\hline MPI & two-stream & emissivity depends on CLW & $\mathrm{x}$ & mixed \\
\hline MRI & delta-Eddington & emissivity depends on optical depth & & random \\
\hline NCAR & delta-Eddington & emissivity depends on fixed CLW & $x$ & random \\
\hline NMC & optics prescribed by cloud height & emissivity $<1$ for high cloud only & & random \\
\hline $\mathbf{N R L}$ & delta-Eddington & emissivity depends on temperature & $\bar{x}$ & mixed \\
\hline $\mathbf{R P N}$ & delta-Eddington & all clouds are blackbody emitters & $\mathrm{x}$ & full \\
\hline SUNYA & delta-Eddington & emissivity depends on CLW & $x$ & random \\
\hline SUNYANCAR & delta-Eddington & emissivity depends on CLW & $\mathrm{x}$ & random \\
\hline UCLA & optics depend on cloud height/depth & emissivity depends on optical depth & $\bar{x}$ & full \\
\hline UGAMP & delta-Eddington & emissivity depends on CLW & $\mathrm{x}$ & mixed \\
\hline UIUC & two-stream and delta-Eddington & emissivity depends on CLW & $\mathrm{x}$ & mixed \\
\hline UKMO & Practical Improved Flux Method & emissivity depends on CLW & $\mathrm{x}$ & mixed \\
\hline YONU & two-stream and delta-Eddington & emissivity depends on CLW & $\mathrm{x}$ & mixed \\
\hline
\end{tabular}


Table 2-10: Convection. The table lists references on schemes used for simulation of deep and shallow convection, with brief descriptions.

\begin{tabular}{|c|c|c|}
\hline AMIP Model & Convection References & Descriptions \\
\hline BMRC & $\begin{array}{l}\text { Kuo (1974), Anthes (1977), } \\
\text { Tiedtke (1988) }\end{array}$ & moisture convergence closure with shallow convection \\
\hline CCC & Boer et al. (1984a) & moist convective adjustment \\
\hline CNRM & Bougeault (1985), Geleyn (1987) & bulk mass flux scheme with shallow convection \\
\hline COLA & Kuo (1965), Sela (1980), Tiedtke (1983) & moisture convergence closure with shallow convection \\
\hline CSIRO & Arakawa (1972), Geleyn (1987) & relaxed moist adjustment with shallow convection \\
\hline $\mathrm{CSU}$ & Arakawa \& Schubert (1974) & interactive cumulus subensembles \\
\hline DERF & Manabe et al. (1965), Tiedtke (1983) & moist convective adjustment with shallow convection \\
\hline DNM & Kuo (1974), Anthes (1977) & moisture convergence closure with moist adjustment \\
\hline ECMWF & Tiedtke (1989), Tiedtke et al. (1988) & bulk mass flux scheme with shallow convection \\
\hline$\widehat{\text { GFDL }}$ & Manabe et al. (1965) & moist convective adjustment \\
\hline GISS & $\begin{array}{l}\text { Del Genio \& Yao (1988), } \\
\text { Yao \& Del Genio (1989) }\end{array}$ & convective plume with entrainment and downdrafts \\
\hline GLA & $\begin{array}{l}\text { Sud et al. (1991) modifications of } \\
\text { Arakawa \& Schubert (1974) }\end{array}$ & $\begin{array}{l}\text { interactive cumulus subensembles with constraints on } \\
\text { critical work function and minimum entrainment rate }\end{array}$ \\
\hline GSFC & Moorthi \& Suarez (1992) & relaxed solution of interactive cumulus subensembles \\
\hline IAP & Arakawa et al. (1969), Zeng et al. (1989) & steady-state cumulus ensemble \\
\hline JMA & modified Kuo (1974), Tiedtke (1983) & moisture convergence closure with $\mathrm{s}$ \\
\hline LMD & Kuo (1965), Manabe \& Strickler (1964) & moisture convergence closure with moist adjustment \\
\hline MGO & Kuo (1974), Meleshko et al. (1991) & moisture convergence closure with moist adjustment \\
\hline MPI & Tiedtke (1989), Tiedtke et al. (1988) & bulk mass flux scheme with shallow convection \\
\hline MRI & $\begin{array}{l}\text { Arakawa \& Schubert (1974), } \\
\text { Tokioka et al. (1988) }\end{array}$ & $\begin{array}{l}\text { interactive cumulus subensembles with constraints on } \\
\text { minimum entrainment rate }\end{array}$ \\
\hline NCAR & Hack (1993) & mass flux scheme applied successively in three layers \\
\hline NMC & Kuo (1965), Sela (1980), Tiedtke (1983) & moisture convergence closure with shallow convection \\
\hline NRL & $\begin{array}{l}\text { modified Arakawa \& Schubert (1974), } \\
\text { Tiedtke (1983) }\end{array}$ & $\begin{array}{l}\text { interactive cumulus subensembles with downdrafts } \\
\text { and shallow convection }\end{array}$ \\
\hline RPN & Kuo (1974), Anthes (1977) & $\begin{array}{l}\text { moisture convergence closure with shallow convection } \\
\text { by generalized boundary-layer turbulence formulation }\end{array}$ \\
\hline SUNYA & Manabe et al. (19G5) & moist convective adjustment \\
\hline SUNYA/NCAR & simplified Kreitzberg \& Perkey (1976) & dry and moist sub-gridscale convective plume model \\
\hline UCLA & Arakawa \& Schubert (1974) & interactive cumulus subensembles \\
\hline UGAMP & Betts \& Miller (1994) & relaxed convective adjustment to reference profiles \\
\hline UIUC & Arakawa \& Schubert (1974) & interactive cumulus subensembles \\
\hline UKMO & Gregory \& Rowntree (1990) & bulk mass flux scheme with updrafts/downdrafts \\
\hline YONU & Arakawa \& Schubert (1974) & interactive cumulus subensembles \\
\hline
\end{tabular}


Table 2-11: Cloud formation and precipitation. The table lists the descriptions or references for the cloud-formation scheme, and whether this is based on prognostic cloud liquid water (CLW). For precipitation, the table lists instances of simulation of autoconversion processes, evaporation in falling, and stochastic spatial variation within a grid box at the surface.

\begin{tabular}{|c|c|c|c|c|c|}
\hline \multirow[b]{2}{*}{ AMIP Model } & \multicolumn{2}{|l|}{ Cloud Formation } & \multicolumn{3}{|c|}{ Precipitation } \\
\hline & Scheme References or Descriptions & $\begin{array}{c}\text { Prognostic } \\
\text { CLW }\end{array}$ & Autocnvrsn & Evaporation & $\begin{array}{l}\text { Stochastic } \\
\text { Variation }\end{array}$ \\
\hline BMRC & Slingo (1987), Rikus (1991) & & & & \\
\hline$\overline{\mathrm{CCC}}$ & McFarlane et al. (1992) & & & & \\
\hline CNRM & Royer et al. (1990), Tiedtke (1984) & & & $\mathrm{x}$ & \\
\hline COLA & Slingo (1987) & & & $\mathrm{x}$ & \\
\hline CSIRO & Rikus (1991), Saito \& Baba (1988) & & & & \\
\hline$\overline{\mathrm{CSU}}$ & Randall et al. (1989) & & & $\mathrm{x}$ & \\
\hline DERF & Gordon (1992) & & & & \\
\hline DNM & Smagorinsky (1965), Galin (1984) & & & & \\
\hline ECMWF & Slingo (1987) & & & $\mathrm{x}$ & \\
\hline GFDL & Wetherald \& Manabe (1988) & & & & \\
\hline GISS & Del Genio et al. (1993) & $\mathrm{x}$ & $\mathrm{x}$ & $\mathrm{x}$ & \\
\hline GLA & Sud \& Walker (1992) & & & $\mathrm{x}$ & \\
\hline GSFC & diagnostic convective, large-scale cloud & & & $\mathrm{x}$ & \\
\hline IAP & Zeng et al. (1989) & & & $\mathrm{x}$ & \\
\hline JMA & Saito \& Baba (1988) & & & & \\
\hline LMD & Le Treut \& Li (1991) & $\mathrm{x}$ & & $\mathrm{x}$ & \\
\hline MGO & Slingo (1987) & & & & \\
\hline MPI & Roeckner et al. (1991) & $\mathrm{x}$ & $\mathrm{x}$ & $\bar{x}$ & \\
\hline MRI & diagnostic convective, large-scale cloud & & & $\mathrm{x}$ & \\
\hline NCAR & Kiehl et al. (1993) & & & & \\
\hline NMC & Slingo (1987) & & & $\mathrm{x}$ & \\
\hline NRL & Slingo \& Ritter (1985), Slingo (1987) & & & $\mathrm{x}$ & \\
\hline $\mathbf{R P N}$ & diagnostic convective, stable cloud & & & $\mathrm{x}$ & \\
\hline SUNYA & Kiehl et al. (1987) & & & & \\
\hline SUNYA/NCAR & Slingo \& Slingo (1991) & & & $x$ & $\mathrm{x}$ \\
\hline UCLA & $\begin{array}{l}\text { diagnostic large-scale, convective, and } \\
\text { boundary-layer cloud }\end{array}$ & & & & \\
\hline UGAMP & Slingo (1987) & & & $\mathrm{x}$ & \\
\hline UIUC & $\mathrm{Oh}(1989)$ & $x$ & $\mathrm{x}$ & $x$ & \\
\hline UKMO & Smith (1990a) & $\mathrm{x}$ & $\mathbf{x}$ & $\mathrm{x}$ & $\mathrm{x}$ \\
\hline YONU & Oh (1989) & $\mathrm{x}$ & $x$ & $\mathrm{x}$ & \\
\hline
\end{tabular}


Table 2-12: Planetary boundary layer (PBL). The table shows whether the PBL depth is a prognostic variable and whether the surface fluxes of momentum, heat, and moisture are stability-dependent. References are also listed on the scheme to determine vertical diffusion from turbulence kinetic energy (TKE), if present.

\begin{tabular}{|c|c|c|c|}
\hline \multirow{2}{*}{ AMIP Model } & \multicolumn{3}{|c|}{ PBL } \\
\hline & Prognostic Depth & Stability-Dep Sfe Flxs & TKE Scheme \\
\hline BMRC & & $\bar{x}$ & \\
\hline CCC & & $\mathrm{x}$ & \\
\hline CNRM & & $\mathrm{x}$ & \\
\hline COLA & & $\mathrm{x}$ & Mellor \& Yamada (1982) \\
\hline CSIRO & & $\bar{x}$ & \\
\hline CSU & $\mathrm{x}$ & $\mathrm{x}$ & Suarez et al. (1983), Randall et al. (1985) \\
\hline DERF & & $\mathrm{x}$ & Mellor \& Yamada (1982) \\
\hline DNM & & $\bar{x}$ & \\
\hline ECMWF & & $\mathrm{x}$ & \\
\hline GFDL & & $\mathrm{x}$ & \\
\hline GISS & & $\mathrm{x}$ & \\
\hline GLA & & $\mathrm{x}$ & Helfand \& Labraga (1988) \\
\hline GSFC & & $\mathrm{x}$ & Helfand \& Labraga (1988) \\
\hline \multicolumn{4}{|l|}{ IAP } \\
\hline JMA & & $\bar{x}$ & Mellor \& Yamada (1974) \\
\hline LMD & & $\mathrm{x}$ & \\
\hline MGO & & $\mathrm{x}$ & \\
\hline MPI & & $\mathbf{x}$ & \\
\hline MRI & $\mathrm{x}$ & $\mathrm{x}$ & Randall (1976) \\
\hline NCAR & & $\mathrm{x}$ & \\
\hline NMC & & $\mathrm{x}$ & \\
\hline NRL & & $\mathrm{x}$ & \\
\hline $\mathbf{R P N}$ & & $\mathrm{x}$ & Benoit et al. (1989) \\
\hline SUNYA & & $x$ & \\
\hline SUNYA/NCAR & & $\mathrm{x}$ & \\
\hline UCLA & $\bar{x}$ & $\overline{\mathrm{x}}$ & Suarez et al. (1983) \\
\hline UGAMP & & $\mathrm{x}$ & \\
\hline UIUC & & $\bar{x}$ & \\
\hline UKMO & & $\mathrm{x}$ & \\
\hline YONU & & $\mathrm{x}$ & \\
\hline
\end{tabular}


Table 2-13: Snow cover and sea ice. The table shows whether snow mass is a prognostic variable, and whether (prognostic or prescribed) snow cover alters the nonradiative thermal properties (heat capacity/ conductivity, etc.) or roughness of the surface. For sea ice, the table shows whether the surface temperature is prognostically determined, and whether there is accumulation of snow on the ice.

\begin{tabular}{|c|c|c|c|c|c|}
\hline \multirow{2}{*}{ AMIP Model } & \multicolumn{3}{|c|}{ Snow Cover } & \multicolumn{2}{|c|}{ Sea Ice } \\
\hline & $\begin{array}{l}\text { Prognostic } \\
\text { Snow Mass }\end{array}$ & $\begin{array}{c}\text { Thermal } \\
\text { Effects }\end{array}$ & $\begin{array}{l}\text { Roughness } \\
\text { Effects }\end{array}$ & $\begin{array}{c}\text { Prognostic Surface } \\
\text { Temperature }\end{array}$ & $\begin{array}{c}\text { Snow } \\
\text { Accumulation }\end{array}$ \\
\hline BMRC & $\bar{x}$ & & & $\bar{x}$ & $\mathrm{x}$ \\
\hline $\mathrm{CCC}$ & $\mathrm{x}$ & $x$ & & $\mathrm{x}$ & $\mathrm{x}$ \\
\hline CNRM & $\mathrm{x}$ & & $\mathrm{x}$ & $\bar{x}$ & $\mathrm{x}$ \\
\hline COLA & $\bar{x}$ & $\mathrm{x}$ & & $\mathrm{x}$ & \\
\hline CSIRO & $x$ & $\bar{x}$ & & $\bar{x}$ & $\mathrm{x}$ \\
\hline$\overline{\mathrm{CSU}}$ & $\mathrm{x}$ & $\bar{x}$ & & $\mathrm{x}$ & \\
\hline DERF & $\mathrm{x}$ & $\mathrm{x}$ & & $\mathrm{x}$ & $\mathrm{x}$ \\
\hline DNM & $\mathrm{x}$ & $\mathrm{x}$ & & $\mathrm{x}$ & $\mathrm{x}$ \\
\hline ECMWF & $\mathrm{x}$ & $\bar{x}$ & & & \\
\hline GFDL & $\mathrm{x}$ & & & $\mathrm{x}$ & $\mathrm{x}$ \\
\hline GISS & $\mathrm{x}$ & $\mathrm{x}$ & & $\bar{x}$ & $\mathrm{x}$ \\
\hline GLA & $\mathrm{x}$ & $\mathrm{x}$ & & $\bar{x}$ & $\mathrm{x}$ \\
\hline GSFC & & $\bar{x}$ & & $\bar{x}$ & \\
\hline IAP & $\mathrm{x}$ & $\bar{x}$ & & $\bar{x}$ & $\mathrm{x}$ \\
\hline JMA & $\bar{x}$ & $\bar{x}$ & $\bar{x}$ & $\bar{x}$ & \\
\hline LMD & $\mathrm{x}$ & $\bar{x}$ & & $\mathrm{x}$ & $\mathrm{x}$ \\
\hline MGO & $\bar{x}$ & $\bar{x}$ & & $\mathrm{x}$ & $\mathrm{x}$ \\
\hline MPI & $\mathrm{x}$ & $\mathrm{x}$ & & $\mathrm{x}$ & \\
\hline MRI & $\mathrm{x}$ & $\mathrm{x}$ & & $\mathrm{x}$ & $\bar{x}$ \\
\hline NCAR & & $\bar{x}$ & $\bar{x}$ & $\mathrm{x}$ & prescribed \\
\hline NMC & $\mathbf{x}$ & & & $\mathrm{x}$ & $\begin{array}{l}\text { with no effects on } \\
\text { ice properties }\end{array}$ \\
\hline NRL & $\bar{x}$ & $\mathrm{x}$ & & $\mathrm{x}$ & \\
\hline$\overline{\mathbf{R P N}}$ & & & & $\mathrm{x}$ & \\
\hline SUNYA & $\mathrm{x}$ & & & $\mathrm{x}$ & \\
\hline SUNYA/NCAR & $\bar{x}$ & $\mathrm{x}$ & $\bar{x}$ & $\mathrm{x}$ & $\bar{x}$ \\
\hline UCLA & $\bar{x}$ & & & $\mathrm{x}$ & \\
\hline UGAMP & $\bar{x}$ & & & & \\
\hline UIUC & $\bar{x}$ & $\mathrm{x}$ & & $\mathrm{x}$ & $\bar{x}$ \\
\hline UKMO & $\mathbf{x}$ & $x$ & $\mathrm{x}$ & $\mathrm{x}$ & $\begin{array}{l}\text { no budget, but snow } \\
\text { cover affects albedo }\end{array}$ \\
\hline YONU & $\bar{x}$ & $\mathrm{x}$ & & $\bar{x}$ & $x$ \\
\hline
\end{tabular}


Table 2-14: Surface characteristics. The table shows whether the surface roughness (or, alternatively, the surface drag coefficient) is spatially variable over ocean, land, or sea ice. The table also shows whether the albedo of any surface depends on solar zenith angle or spectral interval, and whether there is graybody longwave emission (emissivity $\varepsilon<1.0$ ) from any surface.

\begin{tabular}{|c|c|c|c|c|c|c|}
\hline \multirow[b]{2}{*}{ AMIP Model } & \multicolumn{3}{|c|}{ Spatially Variable Roughness } & \multicolumn{2}{|c|}{ Surface Solar Albedo } & \multirow{2}{*}{$\begin{array}{l}\text { Graybody } \\
\text { Emission }\end{array}$} \\
\hline & Ocean & Land & Sea Ice & \begin{tabular}{|l|} 
Zenith-Angle \\
Dependence
\end{tabular} & $\begin{array}{c}\text { Spectral } \\
\text { Dependence }\end{array}$ & \\
\hline BMRC & $\mathrm{x}$ & & & $\mathrm{x}$ & & \\
\hline $\mathrm{CCC}$ & $\mathrm{x}$ & $\mathrm{x}$ & & $\mathrm{x}$ & $\mathrm{x}$ & \\
\hline CNRM & $\mathrm{x}$ & $\bar{x}$ & & $\mathrm{x}$ & & $\mathrm{x}$ \\
\hline COLA & $\bar{x}$ & $\mathrm{x}$ & & $\mathrm{x}$ & $\mathrm{x}$ & \\
\hline CSIRO & $\bar{x}$ & & & $\mathrm{x}$ & & \\
\hline CSU & & $\mathrm{x}$ & & $x$ & $\mathrm{x}$ & \\
\hline DERF & $\mathrm{x}$ & & & $\mathrm{x}$ & & \\
\hline DNM & $\mathrm{x}$ & $\mathrm{x}$ & & & & \\
\hline ECMWF & $\mathrm{x}$ & $x$ & & . & & $\varepsilon$ fixed at 0.996 \\
\hline GFDL & $\mathrm{x}$ & & & $\mathrm{x}$ & & \\
\hline GISS & $\mathrm{x}$ & $\mathrm{x}$ & & $\mathrm{x}$ & $\mathrm{x}$ & $\mathrm{x}$ \\
\hline GLA & $\mathrm{x}$ & $\mathrm{x}$ & & $\mathrm{x}$ & $\mathrm{x}$ & $\mathrm{x}$ \\
\hline GSFC & $\mathrm{x}$ & $\mathrm{x}$ & & $\mathrm{x}$ & & \\
\hline $\mathbf{I A P}$ & $\mathrm{x}$ & $\bar{x}$ & & $\bar{x}$ & & \\
\hline JMA & $\begin{array}{l}\text { only for surface } \\
\text { momentum flux }\end{array}$ & $\mathrm{x}$ & & $x$ & $\mathrm{x}$ & \\
\hline LMD & not specified & $\mathrm{x}$ & & $\mathrm{x}$ & $\mathrm{x}$ & \\
\hline MGO & $x$ & $\mathrm{x}$ & & & & \\
\hline MPI & $x$ & $x$ & & $\mathbf{x}$ & & $\varepsilon$ fixed at 0.996 \\
\hline MRI & & $\begin{array}{c}\begin{array}{c}\text { variable drag } \\
\text { coefficient }\end{array} \\
\end{array}$ & & $\mathrm{x}$ & & \\
\hline NCAR & & $\mathrm{x}$ & & $\mathrm{x}$ & $x$ & \\
\hline NMC & $\bar{x}$ & $\mathrm{x}$ & & $\bar{x}$ & & \\
\hline NRL & $\mathrm{x}$ & $\mathrm{x}$ & $\mathrm{x}$ & $\mathrm{x}$ & & \\
\hline RPN & $\mathrm{x}$ & $\mathrm{x}$ & $\mathrm{x}$ & & & $x$ \\
\hline SUNYA & & & & $x$ & $x$ & \\
\hline SUNYA/NCAR & & $x$ & & $x$ & $x$ & $\mathrm{x}$ \\
\hline UCLA & & $\mathrm{x}$ & & $\mathrm{x}$ & & \\
\hline UGAMP & $\mathrm{x}$ & $\mathrm{x}$ & & $\mathrm{x}$ & & $\varepsilon$ fixed at 0.996 \\
\hline UIUC & $\bar{x}$ & $\bar{x}$ & & $\mathrm{x}$ & & \\
\hline UKMO & $\bar{x}$ & $x$ & ice vs leads & $\mathrm{x}$ & & \\
\hline YONU & $\mathrm{x}$ & $\mathrm{x}$ & & $\mathrm{x}$ & & \\
\hline
\end{tabular}


Table 2-15: Land surface processes. The table lists the number of soil layers for prognostic soil temperature and moisture (0 layers denotes no heat storage, or prescribed moisture); a description of (or references on) the prediction models; and references on the interactive vegetation model, if present.

\begin{tabular}{|c|c|c|c|c|}
\hline \multirow[t]{2}{*}{ AMIP Model } & \multicolumn{2}{|c|}{$\begin{array}{l}\text { Number of } \\
\text { Soil Layers }\end{array}$} & \multirow{2}{*}{$\begin{array}{l}\text { Soil Model Description/References: } \\
\text { Temperature, Moisture }\end{array}$} & \multirow{2}{*}{$\begin{array}{l}\text { Vegetation Model } \\
\text { References }\end{array}$} \\
\hline & Temp. & Moist. & & \\
\hline BMRC & 2 & 1 & prescribed deep temperature, bucket & \\
\hline CCC & 1 & 1 & $\begin{array}{l}\text { Deardorff (1978) force-restore, variable bucket and } \\
\text { evapotranspiration factor }\end{array}$ & \\
\hline CNRM & 2 & 2 & Bhumralkar (1975), Deardorff (1977) force restore & \\
\hline COLA & 2 & 3 & Deardorff (1978) force-restore, diffusion & Xue et al. (1991) \\
\hline CSIRO & 3 & 2 & heat diffusion, Deardorff (1977) force-restore & \\
\hline$\overline{\mathrm{CSU}}$ & 1 & 1 & variable heat capacity, bucket & \\
\hline DERF & 3 & 1 & Deardorff (1978) force-restore, bucket & \\
\hline $\mathbf{D N M}$ & 1 & 1 & prescribed deep temperature/moisture, bucket & \\
\hline ECMWF & 2 & 2 & diffusion of heat/moisture, prescribed deep values & Blondin \& Boettger (1987) \\
\hline GFDL & 0 & 1 & no heat storage, bucket & \\
\hline GISS & $\overline{6}$ & 6 & diffusion of heat, moisture & Abramopoulos et al. (1988) \\
\hline$\overline{\text { GLA }}$ & 2 & 3 & Deardorff (1978) force-restore, diffusion of moisture & Xue et al. (1991) \\
\hline GSFC & 0 & 0 & no heat storage, prescribed moisture & \\
\hline IAP & 1 & 1 & Bhumralkar (1975) force-restore, bucket & \\
\hline JMA & 4 & 3 & Deardorff (1978) force-restore, diffusion & $\begin{array}{l}\text { Sellers et al. (1986), } \\
\text { Sato et al. (1989a, b) }\end{array}$ \\
\hline LMD & 1 & 1 & Corby et al. (1976), bucket & \\
\hline MGO & 3 & 2 & $\begin{array}{l}\text { diffusion of heat, diffusion of moisture with varying } \\
\text { surface field capacity }\end{array}$ & \\
\hline MPI & 5 & 1 & Warrilow et al. (1986), Duemenil \& Todini (1992) & Blondin \& Boettger (1987) \\
\hline MRI & 4 & 4 & diffusion of heat, moisture--cf. Katayama (1978) & \\
\hline NCAR & 4 & 0 & heat diffusion, prescribed wetnesses & \\
\hline NMC & 3 & 1 & heat diffusion, bucket & Pan (1990) \\
\hline NRL & 1 & 0 & relaxation to climatology, prescribed wetnesses & \\
\hline $\mathbf{R P N}$ & 1 & 0 & Deardorff (1978) force-restore,prescribed wetnesses & \\
\hline SUNYA & 0 & 1 & no heat storage, bucket & \\
\hline SUNYA/NCAR & 6 & 6 & diffusion of heat (linear), of moisture (nonlinear) & Pollard \& Thompson (1994 \\
\hline UCLA & 0 & 0 & no heat storage, prescribed wetnesses & \\
\hline UGAMP & 2 & 2 & diffusion of heat/moisture, prescribed deep values & \\
\hline UIUC & 1 & 1 & $\begin{array}{l}\text { Bhumralkar (1975) force-restore,variable-capacity } \\
\text { bucket }\end{array}$ & \\
\hline UKMMO & 4 & 1 & $\begin{array}{l}\text { heat diffusion, single moisture reservoir with } \\
\text { variable hydraulic capacity/conductivity }\end{array}$ & $\begin{array}{l}\text { Warrilow et al. (1986), } \\
\text { Shuttleworth (1988) }\end{array}$ \\
\hline YONU & 1 & 1 & Bhumralkar (1975) force-restore, bucket & \\
\hline
\end{tabular}




\section{Summary Reports}

In this section, some 30 properties are described for each AMIP model. The level of detail of the information is intermediate between that of an outline and a comprehensive documentation of model features. Thus, the intent is to qualitatively summarize the essence of the principal features of a model, rather than to fully elaborate the quantitative details of the algorithms and parameterizations. The latter may be found by examining the documentation cited in the feature descriptions (see the reference bibliography that follows this section), or by consulting the AMIP representatives of the modeling groups of interest.

A strength of this summary documentation is that the features of the AMIP models are elaborated with respect to a common set of categories. These are:

- AMIP representative(s)

- Model designation (following the WGNE-recommended form: institution, model and version, horizontal/vertical resolution)

- Model lineage (predecessor and related models)

- Model documentation (key references)

- Horizontal representation (spectral or finite differences)

- Horizontal resolution

- Vertical domain (lowest/highest atmospheric levels)

- Vertical representation (coordinates and differencing schemes)

- Vertical resolution

- Computer/operating system (for the AMIP simulation)

- Computational performance (minutes per simulated day)

- Initialization (of atmospheric state, snow cover/depth, and soil moisture)

- Time integration scheme(s)

- Smoothing/filling (types of algorithms used)

- Sampling frequency (AMIP history storage interval)

- Atmospheric dynamics (state variables)

- Diffusion (horizontal and vertical)

- Gravity-wave drag 
- Solar constant/cycles (AMIP solar constant, inclusion of diurnal cycle)

- Chemistry (radiatively active gases and aerosols)

- Radiation (shortwave/longwave schemes, cloud-radiative interactions)

- Convection (deep and shallow)

- Cloud formation (prognostic or diagnostic schemes)

- Precipitation (formation and subsequent evaporation)

- Planetary boundary layer (representation and depth)

- Orography (datasets, smoothing procedures)

- Ocean (treatment for AMIP simulation)

- Sea ice (treatment for AMIP simulation)

- Snow cover (formation/melting, effects on surface characteristics)

- Surface characteristics (surface types, roughness, albedo, emissivity)

- Surface fluxes (momentum, heat, and moisture)

- Land surface processes (vegetation and soil thermodynamics/hydrology)

Although not an exhaustive accounting of model features, these categories provide a framework for a reasonably comprehensive description of the numerics, dynamics, and physics of the AMIP models. It is acknowledged, however, that such a schema may convey the false impression that model features which are nonlinearly interconnected are to be viewed as independent entities. In a given feature summary, therefore, the interdependence of model properties is made explicit by directing the reader to examine the descriptions of related features (e.g., by referring to the description of cloud formation in the summary of atmospheric radiation, etc.).

The model feature summary reports follow in the alphabetical order of the AMIP group acronyms (see Table 1-1). 


\title{
BMRC: Bureau of Meteorology Research Centre
}

\author{
AMIP Representative(s) \\ Dr. Bryant McAvaney, Bureau of Meteorology Research Centre, Box 1289K, GPO \\ Melbourne, Victoria 3001, Australia; Phone: +61-3-669-4000; Fax: +61-3-669-4660; \\ e-mail: bma@bom.gov.au
}

Model Designation

BMRC BMRC2.3 R31L9

\section{Model Lineage}

The BMRC model is a descendant of a spectral general circulation model first developed in the 1970s (cf. Bourke et al. 1977, and McAvaney et al. 1978).

\section{Model Documentation}

Key documentation of the BMRC model is provided by Bourke (1988), Hart et al. (1988, 1990), Colman and McAvaney (1991), McAvaney et al. (1991), and Rikus (1991). The model configuration for the AMIP experiment is described by McAvaney and Colman (1993).

\section{Numerical/Computational Properties}

\section{Horizontal Representation}

Spectral (spherical harmonic basis functions) with transformation to a Gaussian grid for calculation of nonlinear quantities and physics.

\section{Horizontal Resolution}

Spectral rhomboidal 31 (R31), roughly equivalent to $2.8 \times 3.8$ degrees latitudelongitude.

\section{Vertical Domain}

Surface to about $9 \mathrm{hPa}$. For a surface pressure of $1000 \mathrm{hPa}$, the lowest atmospheric level is at about $991 \mathrm{hPa}$. 


\section{BMRC}

\section{Vertical Representation}

Conservative finite differences in sigma coordinates.

\section{Vertical Resolution}

There are nine unevenly spaced sigma levels. For a surface pressure of $1000 \mathrm{hPa}$, 3 levels are below $800 \mathrm{hPa}$ and 3 are above $200 \mathrm{hPa}$.

\section{Computer/Operating System}

The AMIP simulation was run on a Cray Y/MP 2E computer using 1 processor in a UNICOS 6.1.6 environment.

\section{Computational Performance}

For the AMIP, about 3 minutes Cray Y/MP computation time per simulation day.

\section{Initialization}

For the AMIP simulation, the model atmosphere is initialized from the ECMWF III-B analysis for 12 UT of 1 January 1979, with nonlinear normal mode initialization operative. Soil moisture is initialized from the January data of Mintz and Serafini (1989). Snow cover is initialized from the albedo data of Hummel and Reck (1979): albedos greater than 40 percent define areas of seasonal snow with initial depth of $5 \mathrm{~m}$; in areas of permanent snow (i.e., Antarctica and Greenland) the initial depth is set to $250 \mathrm{~m}$.

\section{Time Integration Scheme(s)}

A semi-implicit scheme with an Asselin (1972) frequency filter is combined with a split implicit scheme for the vertical diffusion component of the model physics. A time step of 15 minutes is used for both dynamics and physics, except that full calculations of radiative fluxes and heating rates are done once every 3 hours.

\section{Smoothing / Filling}

Orography is smoothed (see Orography). Filling of negative atmospheric moisture values is performed by a combination of local horizontal and vertical borrowing, and global borrowing following the method of Royer (1986). A mass adjustment 
scheme is also used to prevent a slow drift in surface pressure during long integrations. Cf. McAvaney et al. (1991) for further details.

\section{Sampling Frequency}

For the AMIP simulation, the model history is written every 6 hours. (However, fields such as convective and total precipitation are accumulated over a 24-hour period; caution should therefore be exercised in interpreting such fields at subintervals of a day.)

\section{Dynamical/Physical Properties}

\section{Atmospheric Dynamics}

Primitive-equation dynamics are expressed in terms of vorticity, divergence, temperature, surface pressure, and specific humidity.

\section{Diffusion}

- Linear second-order $\left(\nabla^{2}\right)$ horizontal diffusion is applied for wave numbers $n>31$ in the upper part of the spectral rhomboid, with a first-order sigma coordinate correction applied near topography.

- Stability dependent vertical diffusion after Louis (1979) is only applied for sigma levels $>0.5$ in stable layers, but it operates in all unstable layers with no separate removal of dry superadiabats, and with a minimum wind speed difference of $1 \mathrm{~m} \mathrm{~s}^{-1}$ assumed between model levels.

\section{Gravity-wave Drag}

Momentum transports associated with gravity waves are simulated by the method of Palmer et al. (1986), using directionally dependent subgrid-scale orographic variances. Surface stress due to gravity waves excited by stably stratified flow over irregular terrain is calculated from linear theory and dimensional considerations. Gravity-wave stress is a function of atmospheric density, low-level wind, and the Brunt-Vaisalla frequency. The vertical structure of the momentum flux induced by gravity waves is calculated from a local wave Richardson number, which describes the onset of turbulence due to convective instability and the turbulent breakdown approaching a critical level. 
BMRC

\section{Solar Constant/Cycles}

The solar constant is the AMIP-prescribed value of $1365 \mathrm{~W} \mathrm{~m}^{-2}$. Both seasonal and diurnal cycles in solar forcing are simulated.

\section{Chemistry}

Carbon dioxide is assumed to be well mixed at the AMIP-prescribed concentration of $345 \mathrm{ppm}$. Zonally averaged seasonal mean ozone distributions are prescribed from the data of Dopplick (1974), with linear interpolation for intermediate times. No other trace gases or aerosols are present, but the radiative effects of water vapor are included (see Radiation).

\section{Radiation}

- Shortwave Rayleigh scattering and absorption in ultraviolet ( $<0.35$ micron) and visible (0.5-0.7 micron) spectral bands by ozone, and in the near-infrared (0.7-4.0 microns) by water vapor and carbon dioxide follow the method of Lacis and Hansen (1974). Pressure corrections and multiple reflections between clouds are treated. The radiative effects of aerosols are not included directly.

- Longwave radiation follows the simplified exchange method of Fels and Schwarzkopf (1975) and Schwarzkopf and Fels (1991), with slight modifications. (The parent code is compared against benchmark computations by Fels et al. 1991.) Longwave calculations follow the broad-band emissivity approximation in 8 spectral intervals (with wavenumber boundaries at $0,1.6 \times 10^{4}, 5.6 \times 10^{4}, 8.0 \times 10^{4}, 9.0 \times 10^{4}$, $9.9 \times 10^{4}, 1.07 \times 10^{5}, 1.20 \times 10^{5}$, and $2.20 \times 10^{5} \mathrm{~m}^{-1}$ ). Another 14 bands are accounted for in the cooling-to-space corrections. Included in the calculations are Fels and Schwarzkopf (1981) transmission coefficients for carbon dioxide, the water vapor continuum of Roberts et al. (1976), and the effects of water-carbon dioxide overlap and of a Voigt line-shape correction.

- The treatment of cloud-radiative interactions is as described by Rikus (1991) and McAvaney et al. (1991). Shortwave cloud reflectivity/absorptivity is prescribed for ultraviolet-visible and near-infrared spectral bands and depends only on the height class of the cloud (see Cloud Formation). In the longwave, all clouds are assumed to behave as blackbodies (emissivity of 1). For purposes of the radiation calculations, all clouds are assumed to be randomly overlapped in the vertical. 


\section{Convection}

- Deep convection is simulated by a variation of the method of Kuo (1974) that includes modifications of Anthes (1977). Penetrative convection is assumed to occur only in the presence of conditionally unstable layers in the vertical and large-scale net moisture convergence. The convective cloud base is assumed to be at the first level (maximum sigma $=0.926$ ) above the planetary boundary layer $(\mathrm{PBL})$ which is conditionally unstable. The convective cloud is assumed to dissolve instantaneously through lateral mixing, thereby imparting heat and moisture to the environment. In a vertical column the total moisture available from convergence is divided between a fraction $b$ that moistens the environment and the remainder $(1-b)$ that contributes to the latent heating (rainfall) rate. In the Anthes modification of the Kuo scheme, the moistening parameter $\mathrm{b}$ is determined as a cubic function of the ratio of the mean relative humidity of the cloud layer to a prescribed critical relative humidity threshold value; if the cloud relative humidity is less than the threshold, $b$ is set to 1 (no heating of the environment).

- Simulation of shallow convection is parameterized in terms of the model's vertical diffusion scheme, following the method of Tiedtke $(1983,1988)$.

\section{Cloud Formation}

Stratiform cloud formation is based on the relative humidity diagnostic form of Slingo (1987). Clouds are of 3 height classes: high (sigma levels 0.189-0.336), middle (sigma levels 0.500-0.664), and low (sigma levels 0.811-0.926). The fractional amount of each type of cloud is determined from a quadratic function of the difference between the maximum relative humidity of the sigma layer and a threshold relative humidity that varies with sigma level; for high and low cloud the threshold is 60 percent humidity, while for middle cloud it is 50 percent. In addition, following Rikus (1991), low cloud forms when the relative humidity at the lowest atmospheric level (sigma $=0.991$ ) exceeds 60 percent, and is capped by strong static stability in the layer immediately above (i.e., a temperature inversion is present). In this case, the

amount of low cloud increases with the strength of the inversion. (See Convection for the treatment of convective cloud and Radiation for cloud-radiative interactions.) 


\section{BMRC}

\section{Precipitation}

Precipitation from large-scale condensation occurs if the relative humidity exceeds 100 percent. The convective precipitation rate is determined from the variable moistening parameter $\mathrm{b}$ in the Anthes (1977) modification of the Kuo (1974) convection scheme (see Convection). No evaporation of precipitation is simulated. See also Snow Cover.

\section{Planetary Boundary Layer}

The height of the PBL is assumed to be that of the lowest prognostic vertical level (sigma $=0.991$ ). Winds, temperatures, and humidities for calculation of turbulent eddy surface fluxes from bulk formulae are taken to be the same values as those at this lowest atmospheric level (see Surface Fluxes). See also Diffusion and Surface Characteristics.

\section{Orography}

Orography from a $1 \times 1$-degree U.S. Navy dataset is grid-point smoothed using a Cressman (1959) area-averaged weighting function with a radius of influence of 3 degrees for the spectral R31 model resolution (cf. Bourke 1988).

\section{Ocean}

AMIP monthly sea surface temperature fields are prescribed, with values updated every 5 days by linear interpolation.

\section{Sea Ice}

Monthly AMIP sea ice extents are prescribed via a Cressman (1959) weighting function with a 3-degree radius of influence; these monthly ice extents are updated by interpolation every 5 days. The thickness of the sea ice is held fixed at $1 \mathrm{~m}$ for the Antarctic region and $2 \mathrm{~m}$ for the Arctic. Snow is permitted to accumulate or to melt on the ice surface, but there is no conversion of snow to ice. The surface temperature of the sea ice/snow is determined from a heat balance calculation (see Surface Fluxes) with inclusion of a conduction term from the ocean below the ice (at a fixed temperature of $271 \mathrm{~K}$ ) below the ice. 


\section{Snow Cover}

When the weighted average of the air temperature at the lowest two levels (sigma $=0.991$ and 0.926 ) falls below $273.16 \mathrm{~K}$, precipitation falls to the surface as snow. Prognostic snow mass with accumulation and melting over both land and sea ice is modeled. Snow cover affects the surface albedo and the surface roughness (see Surface Characteristics), but there is no explicit allowance for the effects of fractional snow cover. Melting of snow, which occurs when the surface temperature exceeds $0{ }^{\circ} \mathrm{C}$, contributes to soil moisture (see Land Surface Processes), but sublimation of snow is not calculated as part of the surface evaporative flux (see Surface Fluxes).

\section{Surface Characteristics}

- Distinguished surface types include ocean, land, land ice, and sea ice, and the presence of snow cover is also accounted for on the latter three surfaces. Soil or vegetation types are not distinguished.

- The roughness length over oceans is determined from the surface wind stress, following Charnock (1955), with a coefficient of 0.0185 assigned after $\mathrm{Wu}$ (1982); the ocean roughness is constrained to a minimum value of $1.5 \times 10^{-5} \mathrm{~m}$. Roughness lengths are prescribed uniform values over sea ice $(0.001 \mathrm{~m})$ and land surfaces $(0.168 \mathrm{~m})$, but the presence of snow cover changes the roughness to a new (fixed) value.

- Over oceans, the surface albedo depends on solar zenith angle, following Payne (1972). Seasonal climatological surface albedos of Hummel and Reck (1979) are prescribed over land. The surface albedos of sea ice and snow-covered land follow the temperature-ramp formulation of Petzold (1977), with different values of albedo limits and a lower temperature range for sea ice and snow, as described by Colman and McAvaney (1992).

- Longwave emissivity is set to unity for all surfaces (i.e., blackbody emission is assumed).

Surface Fluxes

- Surface solar absorption is determined from surface albedos, and longwave emission from the Planck equation with prescribed constant surface emissivity of 1.0 (see Surface Characteristics).

- The surface turbulent eddy fluxes of momentum, heat, and moisture follow 


\section{BMRC}

Monin-Obukhov similarity theory, and are formulated in terms of bulk formulae with stability-dependent drag/transfer coefficients determined as in Louis (1979). The momentum flux is given by the product of the air density, a neutral drag coefficient, wind speed and wind vector at the lowest prognostic level (sigma $=0.991$ ), and a transfer function that depends on roughness length (see Surface Characteristics) and stability (bulk Richardson number). Surface wind speed is constrained to a minimum of $1 \mathrm{~m} \mathrm{~s}^{-1}$. The flux of sensible heat is given by a product of a neutral exchange coefficient, the wind speed at the lowest prognostic level, the difference in temperatures between the ground and the first prognostic atmospheric level, and a modified form of the transfer function for unstable conditions (cf. Louis 1979).

- The flux of surface moisture is given by a product of the same transfer coefficient and stability function as for sensible heat, an evapotranspiration efficiency $\beta$, and the difference between the specific humidity at the first prognostic level and the saturation specific humidity at the surface temperature and pressure. For calm conditions over the oceans, evaporation also is enhanced following the approximation of Miller et al. (1992) for the transfer coefficient. Over oceans, sea ice, and snow $\beta$ is prescribed to be unity; over land $\beta$ is a function of the ratio of soil moisture to a constant field capacity (see Land Surface Processes).

\section{Land Surface Processes}

- Soil temperature is computed from heat storage in two layers with a climatological temperature specified in a deeper layer. The upper boundary condition is the surface energy balance (see Surface Fluxes). The heat conductivity of soil is fixed under all conditions.

- Prognostic soil moisture is represented by a single-layer "bucket" model with uniform field capacity of $0.15 \mathrm{~m}$ after Manabe and Holloway (1975). Both precipitation and snowmelt contribute to soil moisture. The evapotranspiration efficiency $\beta$ (see Surface Fluxes) is a function of the ratio of soil moisture to the field capacity. Runoff occurs implicitly if this ratio exceeds unity. 


\section{CCC: Canadian Centre for Climate Research}

\section{AMIP Representative(s)}

Dr. George Boer and Dr. Norman McFarlane, Canadian Centre for Climate Research, Atmospheric Environment Service, University of Victoria, P.O. Box 1700 MS 3339, Victoria, British Columbia V8W 2Y2, Canada; Phone: +1-604-363-8227; Fax: +1604-363-8247; e-mail: gboer@uvic.bc.doe.ca (Boer) and nmcfarlane@uvic.bc.doe.ca (McFarlane)

\section{Model Designation \\ CCC GCMII T32L10}

\section{Model Lineage}

The CCC model is the second-generation version of a model first developed in the early 1980s for climate applications.

\section{Model Documentation}

Key papers by McFarlane et al. (1992) and Boer et al. (1992) describe the features and equilibrium climate of the CCC model, and its simulation of greenhouse gas-induced climate change. Some properties remain the same as those of the firstgeneration CCC model documented by Boer et al. (1984a, b).

\section{Numerical/Computational Properties}

\section{Horizontal Representation}

Spectral (spherical harmonic basis functions) with transformation to a Gaussian grid for calculation of nonlinear quantities and some physics.

\section{Horizontal Resolution}

Spectral triangular 32 (T32), roughly equivalent to $3.75 \times 3.75$ degrees latitudelongitude. 
CCC

\section{Vertical Domain}

Surface to $5 \mathrm{hPa}$. For a surface pressure of $1000 \mathrm{hPa}$, the lowest atmospheric level is at a pressure of about $980 \mathrm{hPa}$.

\section{Vertical Representation}

Piecewise finite-element formulation of hybrid coordinates (cf. Laprise and Girard 1990).

\section{Vertical Resolution}

There are 10 irregularly spaced hybrid levels. For a surface pressure of $1000 \mathrm{hPa}$, 3 levels are below $800 \mathrm{hPa}$ and 4 levels are above $200 \mathrm{hPa}$.

\section{Computer/Operating System}

The AMIP simulation was run on the Cray X/MP computer of the Canadian Meteorological Centre (in Dorval, Quebec) using a single processor in a COS 1.17 environment.

\section{Computational Performance}

For the AMIP, about 6 minutes Cray XMP computation time per simulation day.

\section{Initialization}

For the AMIP simulation, the model atmosphere is initialized from FGGE III-B observational analyses for 1 January 1979. Soil moisture and snow cover/depth are initialized from January mean values obtained from an earlier multiyear model simulation.

\section{Time Integration Scheme(s)}

A semi-implicit time integration scheme with an Asselin (1972) frequency filter is used. The time step is 20 minutes for dynamics and physics, except for full calculations of radiative fluxes and heating rates. Shortwave radiation is calculated every 3 hours, and longwave radiation every 6 hours, with interpolated values used at intermediate time steps (cf. McFarlane et al. 1992). 


\section{Smoothing / Filling}

Orography is truncated at spectral T32 (see Orography). Negative values of atmospheric specific humidity (which arise because of numerical truncation errors in the discretized moisture equation) are filled in a two-stage process. First, all negative values of specific humidity are made slightly positive by borrowing moisture (where possible) from other layers in the same column. If column moisture is insufficient, a nominal minimum bound is imposed, the moisture deficit is accumulated over all atmospheric points, and the global specific humidity is reduced proportionally. This second stage is carried out in the spectral domain (cf. McFarlane et al. 1992).

\section{Sampling Frequency}

For the AMIP simulation, the model history is written every 6 hours. (However, some archived variables, including most of the surface quantities, are accumulated rather than sampled.)

\section{Dynamical/Physical Properties}

\section{Atmospheric Dynamics}

Primitive-equation dynamics are expressed in terms of vorticity, divergence, temperature, the logarithm of surface pressure, and specific humidity.

\section{Diffusion}

- Horizontal diffusion follows the scale-dependent eddy viscosity formulation of Leith (1971) as described by Boer et al. (1984a). Diffusion is applied to spectral modes of divergence, vorticity, temperature, and moisture, with total wavenumbers $>18$ on hybrid vertical surfaces.

- Second-order vertical diffusion of momentum, moisture, and heat operates above the surface. The vertically varying diffusivity depends on stability (gradient Richardson number) and the vertical shear of the wind, following standard mixinglength theory. Diffusivity for moisture is taken to be the same as that for heat. Cf. McFarlane et al. (1992) for details. See also Surface Fluxes. 


\section{$\mathrm{CCC}$}

\section{Gravity-wave Drag}

Simulation of subgrid-scale gravity-wave drag follows the parameterization of McFarlane (1987). Deceleration of the resolved flow by dissipation of orographically excited gravity waves is a function of the rate at which the parameterized vertical component of the gravity-wave momentum flux decreases in magnitude with height. This momentum-flux term is the product of local air density, the component of the local wind in the direction of that at the near-surface reference level, and a displacement amplitude. At the surface, this amplitude is specified in terms of the mesoscale orographic variance, and in the free atmosphere by linear theory, but it is bounded everywhere by wave saturation values. See also Orography.

\section{Solar Constant / Cycles}

The solar constant is the AMIP-prescribed value of $1365 \mathrm{~W} \mathrm{~m}^{-2}$. Both seasonal and diurnal cycles in solar forcing are simulated.

\section{Chemistry}

The carbon dioxide concentration is the AMIP-prescribed value of $345 \mathrm{ppm}$. A monthly zonally averaged ozone distribution from data by Wilcox and Belmont (1977) is specified. Radiative effects of water vapor also are treated (see Radiation).

\section{Radiation}

- Shortwave radiation is modeled after an updated scheme of Fouquart and Bonnel (1980). Upward/downward shortwave irradiance profiles are evaluated in two stages. First, a mean photon optical path is calculated for a scattering atmosphere including clouds, aerosols, and gases. The reflectance and transmittance of these elements are calculated by, respectively, the delta-Eddington method (cf. Joseph et al. 1976) and by a simplified two-stream approximation. The scheme evaluates upward/downward shortwave fluxes for two reference cases: a conservative atmosphere and a firstguess absorbing atmosphere; the mean optical path is then computed for each absorbing gas from the logarithm of the ratio of these reference fluxes. In the second stage, final upward/downward fluxes are computed for visible (0.30-0.68 micron) and nearinfrared (0.68-4.0 microns) spectral intervals using more exact gas transmittances (cf. Rothman 1981), and with adjustments made for the presence of clouds. The asymmetry factor is prescribed for clouds, and the optical depth and single-scattering albedo 
are functions of cloud liquid water content (cf. Betts and Harshvardhan 1987) and ice crystal content (cf. Heymsfield 1977).

- Longwave radiation is modeled in six spectral intervals between wavenumbers 0 to $2.82 \times 10^{5} \mathrm{~m}^{-1}$ after the method of Morcrette $(1984,1990,1991)$, which corrects for the temperature/pressure dependence of longwave absorption by gases and aerosols. Longwave absorption in the water vapor continuum follows Clough et al. (1980). Clouds are treated as graybodies in the longwave, with emissivity depending on optical depth (cf. Platt and Harshvardhan 1988), and with longwave scattering by cloud droplets neglected. The effects of cloud overlap in the longwave are treated following a modified scheme of Washington and Williamson (1977): upward/downward irradiances are computed for clear-sky and overcast conditions, and final irradiances are determined from a linear combination of these extreme cases weighted by the actual partial cloudiness in each vertical layer. For purposes of the radiation calculations, clouds occupying adjacent layers are assumed to be fully overlapped, but to be randomly overlapped otherwise. Cf. McFarlane et al. (1992) for further details.

\section{Convection}

A moist convective adjustment procedure is applied on pairs of vertical layers whenever the model atmosphere is conditionally unstable. Convective instability occurs when the local thermal lapse rate exceeds a critical value, which is determined from a weighted linear combination of dry and moist adiabatic lapse rates, where the weighting factor (with range 0 to 1 ) is a function of the local relative humidity. Convective instability may occur in association with condensation of moisture under supersaturated conditions, and the release of precipitation and associated latent heat (see Precipitation). Cf. Boer et al. (1984a) for further details.

\section{Cloud Formation}

The fractional cloud cover in a vertical layer is computed from a linear function of the relative humidity excess above a threshold value. The threshold is a nonlinear function of height for local sigma levels $>0.5$, and is a constant 85 percent relative humidity at higher altitudes. (Note that the cloud scheme uses locally representative sigma coordinates, while other model variables use hybrid vertical coordinates--see Vertical Representation). To prevent development of excessive low cloudiness, no 


\section{$\mathrm{CCC}$}

clouds are allowed in the lowest model layer. Cf. McFarlane et al. (1992) for further details. See also Radiation for treatment of cloud-radiative interactions.

\section{Precipitation}

Condensation and precipitation occur under conditions of local supersaturation, which are treated operationally as part of the model's convective adjustment scheme (see Convection). All the precipitation falls to the surface without subsequent evaporation to the surrounding atmosphere. See also Snow Cover.

\section{Planetary Boundary Layer}

The depth of the PBL is not explicitly determined, but in general is assumed to be greater than that of the surface layer (centered at the lowest prognostic vertical level--about $980 \mathrm{hPa}$ for a surface pressure of $1000 \mathrm{hPa}$ ). The PBL depth is affected by dry convective adjustment (see Convection), which simulates boundary-layer mixing of heat and moisture, and by enhanced vertical diffusivities (see Diffusion), which may be invoked in the lowest few layers that are determined to be convectively unstable (cf. Boer et al. 1984a). Within the surface layer of the PBL, temperature and moisture required for calculation of surface fluxes are assigned the same values as those at the lowest level, but the wind is taken as one-half its value at this level (see Surface Fluxes).

\section{Orography}

Orographic heights with a resolution of 10 minutes arc on a latitude/longitude grid are smoothed by averaging over 1.8-degree grid squares, and the orographic variance about the mean for each grid box also is computed (see Gravity-Wave Drag). These means and variances are interpolated to a slightly coarser Gaussian grid (64 longitudes $\mathrm{x} 32$ latitudes), transformed to the spectral representation, and truncated at the model resolution (spectral T32).

\section{Ocean}

AMIP monthly sea surface temperature fields are prescribed, with daily values determined by linear interpolation. 
Sea Ice

AMIP monthly sea ice extents are prescribed. Snow may accumulate on sea ice (see Snow Cover). The surface temperature of the ice is a prognostic function of the surface heat balance (see Surface Fluxes) and of a heat flux from the ocean below. This ocean heat flux depends on the constant ice thickness and the temperature gradient between the ocean and the ice.

\section{Snow Cover}

If the near-surface air temperature is $<0{ }^{\circ} \mathrm{C}$, precipitation falls as snow. Prognostic snow mass is determined from a budget equation, with accumulation and melting treated over both land and sea ice. Snow cover affects the surface albedo of land and of sea ice, as well as the heat capacity of the soil. Sublimation of snow is calculated as part of the surface evaporative flux. Melting of snow, as well as melting of ice interior to the soil, contributes to soil moisture. Cf. McFarlane et al. (1992) for further details. See also Surface Characteristics, Surface Fluxes, and Land Surface Processes.

\section{Surface Characteristics}

- Local roughness lengths are derived (cf. Boer et al. 1984a) from prescribed neutral surface drag coefficients (see Surface Fluxes).

- The 1 x 1-degree Wilson and Henderson-Sellers (1985) data on $24 \mathrm{soil} /$ vegetation types are used to determine the most frequently occurring primary and secondary types (weighted $2 / 3$ vs $1 / 3$ ) for each grid box. Averaged local soil/vegetation parameters include field capacity and slope factor for predicting soil moisture (see Land Surface Processes), and snow masking depth for the surface albedo (see below). These are obtained by table look-up based on primary/secondary vegetation types.

- Over bare dry land, the surface background albedo is determined from a weighted average for each of 24 vegetation types in the visible (0.30-0.68 micron) and near-infrared (0.68-4.0 microns) spectral bands; for wet soil, albedos are reduced up to 0.07 . For vegetated surfaces, albedos are determined from a $2 / 3$ vs $1 / 3$ weighting of albedos of the local primary/secondary vegetation types. The local land albedo also depends on the fractional snow cover and its age (fractional coverage of a grid box is given by the ratio of the snow depth to the specified local masking depth); the resulting albedo is a linear weighted combination of snowcovered and snow-free albedos. Over the oceans, latitude-dependent albedos 


\section{$\mathrm{CCC}$}

which range between 0.06 and 0.17 are specified independent of spectral interval. The background albedos for sea ice are 0.55 in the near-infrared and 0.75 in the visible; these values are modified by snow cover, puddling effects of melting ice (a function of mean surface temperature), and by the fraction of ice leads (a specified function of ice mass).

- The longwave emissivity is prescribed as unity (i.e., blackbody emission is assumed) for all surfaces. Cf. McFarlane et al. (1992) for further details.

\section{Surface Fluxes}

- The surface solar absorption is determined from surface albedos, and the longwave emission from the Planck equation with prescribed emissivity of 1.0 (see Surface Characteristics).

- The surface turbulent eddy fluxes of momentum, heat, and moisture are expressed as bulk formulae following Monin-Obukhov similarity theory. The momentum flux is a product of a neutral drag coefficient, the surface wind speed and wind vector (see Planetary Boundary Layer), and a function of stability (bulk Richardson number). Drag coefficients over land and ice are prescribed after Cressman (1960), but over the oceans they are a function of surface wind speed (cf. Smith 1980). The flux of sensible heat is a product of a neutral transfer coefficient, the surface wind speed, the difference in temperatures between the surface and that of the lowest atmospheric level, and the same stability function as for the momentum flux. (The transfer coefficient has the same value as the drag coefficient over land and ice, but is not a function of surface wind over the oceans.)

- The flux of surface moisture is a product of the same transfer coefficient and stability function as for sensible heat, an evapotranspiration efficiency $\beta$, and the difference between the specific humidity at the lowest atmospheric level (see Planetary Boundary Layer) and the saturation specific humidity at the temperature/pressure of the surface. Over the oceanis and sea ice, $\beta$ is prescribed as 1 ; over snow, it is the lesser of 1 or a function of the ratio of the snow mass to a critical value $\left(10 \mathrm{~kg} \mathrm{~m}^{-2}\right)$. Over land, $\beta$ depends on spatially varying soil moisture and field capacities (see Land Surface Processes), and on slope factors for primary/secondary vegetation and soil types (see Surface Characteristics). For grid boxes with fractional snow coverage, a composite $\beta$ is obtained from a weighted linear combination of snow-free and snow-covered values. Cf. Boer et al. (1984a) and McFarlane et al. (1992) for further details. 


\section{Land Surface Processes}

- Soil heat storage is determined as a residual of the surface heat fluxes and of the heat source/sink of freezing/melting snow cover and soil ice (see below). Soil temperature is computed from this heat storage in a single layer, following the method of Deardorff (1978) which accounts for both diurnal and longer-period forcing. The composite conductivity/heat capacity of the soil in each grid box is computed as a function of soil type, soil moisture, and snow cover.

- Soil moisture is predicted by a single-layer "bucket" model with field capacity and slope factors varying by primary/secondary soil and vegetation types for each grid box (see Surface Characteristics). Soil moisture budgets include both liquid and frozen water. The effective local moisture capacity is given by the product of field capacity and slope factor, with evapotranspiration efficiency $\beta$ a function of the ratio of soil moisture to the local effective moisture capacity (see Surface Fluxes). Runoff occurs implicitly if this ratio exceeds 1 (which is more likely the higher the local slope factor and the lower the local field capacity). Cf. McFarlane et al. (1992) and Boer et al. (1984a) for further details. 


\section{CNRM: Centre National de Recherches Météorologiques}

\section{AMIP Representative(s)}

Dr. Jean-Francois Mahfouf and Dr. Daniel Cariolle, Centre National de Recherches Météorologiques, 42 Avenue Coriolis, 31057 Toulouse, France; Phone: +33-61-07-96-35; Fax: +33-61-07-96-00; e-mail: mahfouf@meteo.fr

Model Designation

CNRM EMERAUDE T42L30

\section{Model Lineage}

The CNRM model is derived from a previously operational French weather forecast model, EMERAUDE (cf. Coiffier et al. 1987 and Geleyn et al. 1988), but with adaptations made for climate simulation.

\section{Model Documentation}

Key documentation of atmospheric model features is provided by Bougeault (1985), Cariolle and Déqué (1986), Cariolle et al. (1990), Clary (1987), Geleyn (1987), Geleyn and Preuss (1983), Ritter and Geleyn (1992), and Royer et al. (1990). The surface schemes follow the methods of Bhumralkar (1975) and Deardorff $(1977,1978)$.

\section{Numerical/Computational Properties}

\section{Horizontal Representation}

Spectral (spherical harmonic basis functions) with transformation to a Gaussian grid for calculation of nonlinear quantities and some physics.

\section{Horizontal Resolution}

Spectral triangular 42 (T42), roughly equivalent to $2.8 \times 2.8$ degrees latitudelongitude. 


\section{Vertical Domain}

Surface to $0.01 \mathrm{hPa}$. For a surface pressure of $1000 \mathrm{hPa}$, the lowest atmospheric level is at about $995 \mathrm{hPa}$.

\section{Vertical Representation}

Finite differences in hybrid sigma-pressure coordinates (cf. Simmons and Burridge 1981). Above $165 \mathrm{hPa}$ all levels are in constant pressure coordinates (cf. Cariolle et al. 1990).

\section{Vertical Resolution}

There are 30 unevenly spaced hybrid sigma-pressure levels (see Vertical Representation). For a surface pressure of $1000 \mathrm{hPa}, 4$ levels are below $800 \mathrm{hPa}$ and 20 levels are above $200 \mathrm{hPa}$ (cf. Cariolle et al. 1990).

\section{Computer/Operating System}

The AMIP simulation was run on a Cray 2 computer, using a single processor in the UNICOS environment.

\section{Computational Performance}

For the AMIP experiment, about 15 minutes Cray 2 computation time per simulated day.

\section{Initialization}

For the AMIP experiment, the model atmosphere, soil moisture, and snow cover/ depth are initialized for 1 January 1979 from a previous model simulation.

\section{Time Integration Scheme(s)}

A semi-implicit scheme with an Asselin (1972) frequency filter is used with a time step of 15 minutes for integration of dynamics and physics, including full calculation of shortwave and longwave radiative fluxes (see Radiation). 


\section{CNRM}

\section{Smoothing / Filling}

Orography is area-averaged on the Gaussian grid (see Orography). Filling of negative values of atmospheric moisture follows the global horizontal borrowing scheme of Royer (1986), which ensures conservation of total moisture in each vertical layer.

\section{Sampling Frequency}

For the AMIP simulation, the history of selected variables is written every 6 hours.

\section{Dynamical/Physical Properties}

\section{Atmospheric Dynamics}

Primitive-equation dynamics are expressed in terms of vorticity and divergence, temperature, specific humidity, and surface pressure. Ozone is also a prognostic variable (see Chemistry).

\section{Diffusion}

- Linear fourth-order $\left(\nabla^{4}\right)$ horizontal diffusion is applied on hybrid sigmapressure surfaces to vorticity, divergence, temperature, and specific humidity. The diffusion coefficient is a prescribed function of height.

- Stability-dependent vertical diffusion of momentum, heat, and moisture after Louis et al. (1981) is applied at levels up to $25 \mathrm{hPa}$. The diffusion coefficients depend on the bulk Richardson number and, following standard mixing-length theory, the vertical wind shear.

\section{Gravity-wave Drag}

Gravity-wave drag is parameterized after the linear method of Clary (1987), which assumes that subgrid-scale orographic variances generate a continuous spectrum of bidirectional gravity waves (see Orography). The momentum flux induced by a gravity wave extends vertically up to a critical absorption level (where the local wind becomes orthogonal to the flux vector). 


\section{Solar Constant / Cycles}

The solar constant is the AMIP-prescribed value of $1365 \mathrm{~W} \mathrm{~m}^{-2}$. Both seasonal and diurnal cycles in solar forcing are simulated.

\section{Chemistry}

The carbon dioxide concentration is the AMIP-prescribed value of $345 \mathrm{ppm}$. Ozone concentrations are prognostically determined from a transport equation with linearized photochemical sources and sinks and relaxation coefficients calculated from a two-dimensional photochemical model (cf. Cariolle and Déqué 1986 and Cariolle et al. 1990). Radiative effects of water vapor, oxygen, nitrous oxide, methane, carbon monoxide, and of a globally averaged mixed-aerosol profile also are treated (see Radiation).

\section{Radiation}

- Radiation is modeled by the scheme of Ritter and Geleyn (1992). All flux calculations follow the delta-two-stream approach (cf. Zdunkowski et al. 1980, 1982) applied in one shortwave interval between 0.25 and 4.64 microns, and in one longwave interval between 4.64 and 104.5 microns. Differential fluxes are calculated by subdividing the atmosphere into layers of constant optical properties (optical depth, single-scattering albedo, asymmetry factor) with linear relationships assumed (cf. Geleyn and Hollingsworth 1979). Optical properties are specified after Rothman et al. (1983) for gases, after Tanré et al. (1983) for five types of aerosol, and after Stephens (1979) for water clouds with eight different droplet-size distributions that are related to diagnostic cloud liquid water content (LWC) following Betts and Harshvardhan (1987). Optical properties of ice clouds are not specifically included.

- Gaseous optical depths are first evaluated with band-model calculations along idealized photon paths, and then are reused in multiple scattering calculations for both shortwave and longwave fluxes in a manner similar to that of Geleyn and Hollingsworth (1979). Continuum absorption is treated by including a special term in the equivalent width for a modified Malkmus (1967) model.

- Partial cloudiness in each layer is treated by specifying separate sets of optical properties and fluxes for the cloudy and cloud-free portions. Cloud layers are assumed to overlap randomly in the vertical. See also Cloud Formation. 


\section{CNRM}

\section{Convection}

- The effects of sub-gridscale cumulus convection on the gridscale heat and water budgets are represented by the bulk mass flux scheme of Bougeault (1985). The cloud profile is determined from a moist adiabat, with incorporation of entrainment of environmental air. The scheme also predicts the convective mass inside the cloud, assuming the vertical mass flux profile varies as the square root of the moist static energy excess (with a proportionality coefficient determined after Kuo 1965 from the large-scale moisture convergence and turbulent water transport at the cloud base). Convective detrainment is proportional to the excess of cloud temperature and moisture over their environmental values (the detrainment coefficient being determined from conservation of moist static energy in the column). The convective precipitation rate is given by the difference between the total moisture convergence and the environmental moistening due to detrainment, under the assumption of no evaporation of precipitation below the cloud base (see Precipitation).

- Following Geleyn (1987), shallow convection is accounted for by modifying the bulk Richardson number to include the gradient of specific humidity deficit in computing vertical stability.

\section{Cloud Formation}

The stratiform cloud fraction is determined from a quadratic function of the relative humidity excess over a prescribed critical humidity profile that is a nonlinear function of pressure (cf. Royer et al. 1990). In addition, the stratiform fraction is not allowed to exceed 0.5 in each layer. The total convective cloud cover, determined as a linear function of convective precipitation after Tiedtke (1984), is distributed uniformly in the vertical. See also Radiation for cloud-radiative interactions.

\section{Precipitation}

Precipitation is produced by the convective scheme (see Convection) and by large-scale condensation under supersaturated conditions. Subsequent evaporation of large-scale precipitation in nonsaturated lower layers follows the parameterization of Kessler (1969). There is no evaporation of convective precipitation below the cloud base. 


\section{Planetary Boundary Layer}

There is no special parameterization of the PBL other than the representation of stability-dependent vertical diffusion of momentum, heat, and moisture (see Diffusion and Surface Fluxes).

\section{Orography}

Raw orography obtained from the U.S. Navy dataset with resolution of 10 minutes arc (cf. Joseph 1980) is area-averaged on the Gaussian grid, transformed to spectral space, and truncated at T42 resolution. Subgrid-scale orographic variances required for the gravity-wave drag parameterization are computed from the same dataset (see Gravity-wave Drag).

\section{Ocean}

AMIP monthly sea surface temperatures are prescribed, with daily values determined by linear interpolation.

Sea Ice

AMIP monthly sea ice extents are prescribed. The surface temperature of the ice is determined from a balance of energy fluxes (see Surface Fluxes) that includes conduction from the ocean below. The conduction flux is obtained by the Deardorff (1978) force-restore method, where the restore temperature is the ice melting point and the thermal inertia is modified from that used over land surfaces. Accumulated snow modifies the albedo, but not the thermal properties of the ice. See also Snow Cover and Surface Characteristics.

\section{Snow Cover}

If the surface air temperature is $<0^{\circ} \mathrm{C}$, precipitation falls as snow. Prognostic snow mass is determined from a budget equation, with accumulation and melting included over both land and sea ice. Snow cover affects the albedo and roughness of the surface (see Surface Characteristics), but not the heat capacity/conductivity of soil or sea ice. Sublimation of snow is calculated as part of the surface evaporative flux (see Surface Fluxes), and snowmelt contributes to soil moisture (see Land Surface Processes). 


\section{CNRM}

\section{Surface Characteristics}

- The surface roughness length over the oceans is prognostically determined from the wind stress after the Charnock (1955) relation with a coefficient of 0.19 . The roughness length over ice surfaces is specified as a constant $1 \times 10^{-3} \mathrm{~m}$. Over land, the surface roughness is a function of the variance of the orography and vegetation cover that is prescribed from data of Baumgartner et al. (1977). The roughness length of land and ice surfaces also varies with snow depth.

- Surface albedos are prescribed from monthly satellite data of Geleyn and Preuss (1983). The albedos are also a function of solar zenith angle, but not spectral interval. Prognostic snow cover modifies the albedo of land and ice surfaces according to the depth of snow.

- Longwave emissivity is specified from CLIMAP data for all surfaces.

\section{Surface Fluxes}

- The surface solar absorption is determined from surface albedos, and longwave emission from the Planck equation with prescribed emissivities (see Surface Characteristics).

- In the lowest atmospheric layer, turbulent eddy fluxes of momentum, heat, and moisture follow Monin-Obukov theory, and are expressed as bulk formulae multiplied by drag or transfer coefficients that depend on stability (bulk Richardson number) and surface roughness length (see Surface Characteristics) after the formulation of Louis et al. (1981). The surface wind, temperature, and humidity required for the bulk formulae are taken to be the values at the lowest atmospheric level (at sigma $=0.99527$, or about $40 \mathrm{~m}$ above the ground), and the same transfer coefficient is used for the heat and moisture fluxes.

- The effective ground value of humidity also required for determination of the surface moisture flux is obtained as a fraction $\alpha$ of the saturated humidity at the ground temperature; $\alpha$ is unity over oceans, snow, and ice, but it is a function of the surface soil moisture over land (see Land Surface Processes).

- Above the surface layer, turbulent eddy fluxes are represented as stabilitydependent diffusive processes following the method of Louis et al. (1981)--see Diffusion. 


\section{Land Surface Processes}

- Soil temperature is prognostically determined in two layers after the method of Bhumralkar (1975) with time constants of 1 day and 5 days, respectively. Relaxation (with time constant 21 days) toward a climatological deep soil temperature is also imposed, while the boundary condition at the soil-atmosphere interface is the net balance of the surface energy fluxes (see Surface Fluxes). Soil heat capacity/ conductivity is spatially invariant and is not affected by snow cover.

- Soil moisture is prognostically determined by the force-restore method of Deardorff (1977) in two layers: a shallow surface reservoir of capacity $0.02 \mathrm{~m}$ to capture diurnal variations, and an underlying reservoir of $0.10 \mathrm{~m}$ capacity to simulate the effects of longer-term variations. Both precipitation and snowmelt contribute to soil moisture, while evaporation depletes it. The fraction $\alpha$ of ground saturation humidity that is available for evaporation (see Surface Fluxes) is determined from an empirical function of the ratio of soil moisture in the shallow upper layer to its saturation value. Runoff occurs if soil moisture exceeds the maximum capacity for each layer. 


\section{COLA: Center for Ocean-Land-Atmosphere Studies}

\section{AMIP Representative(s)}

Dr. David Straus, Center for Ocean-Land-Atmosphere Studies, 4041 Powder Mill Road, Suite 302, Calverton, Maryland 20705-3106; Phone: +1-301-595-7000 or +1-301-902-1255; Fax: +1-301-595-9793; e-mail: straus@cola.iges.org

\section{Model Designation}

COLA COLA1.1 R40L18

\section{Model Lineage}

The COLA model is derived from the NMC Medium-Range Forecast (MRF) model (cf. NMC Development Division 1988), but with substantial modifications in the treatment of vertical diffusion, radiation and cloud-radiative interactions, surface characteristics and fluxes, and land-surface processes.

\section{Model Documentation}

Key documentation of the basic model framework is provided by Kinter et al. (1988), with subsequent modifications described by Sato et al. (1989a,b), Xue et al. (1991), and Hou (1991).

\section{Numerical/Computational Properties}

\section{Horizontal Representation}

Spectral (spherical harmonic basis functions) with transformation to a Gaussian grid for calculation of nonlinear quantities and physics.

\section{Horizontal Resolution}

Spectral rhomboidal 40 (R40), roughly equivalent to $1.8 \times 2.8$ degrees latitudelongitude. 


\section{Vertical Domain}

Surface to $10 \mathrm{hPa}$. For a surface pressure of $1000 \mathrm{hPa}$, the lowest atmospheric level is at a pressure of about $995 \mathrm{hPa}$.

\section{Vertical Representation}

Finite-difference sigma coordinates.

Vertical Resolution

There are 18 unevenly spaced sigma levels. For a surface pressure of $1000 \mathrm{hPa}$, 5 levels are below $800 \mathrm{hPa}$ and 4 levels are above $200 \mathrm{hPa}$.

Computer/Operating System

The AMIP simulation was run on a Cray 2 computer using a single processor in CTSS and UNICOS environments.

\section{Computational Performance}

For the AMIP experiment, about 18 minutes Cray 2 computation time per simulation day.

\section{Initialization}

For the AMIP experiment, the model atmospheric state is initialized from the NMC analysis for 1 January 1979, with nonlinear normal mode initialization also performed (cf. Machenauer 1977). January soil moisture and snow cover/depth are obtained from GFDL climatologies.

\section{Time Integration Scheme(s)}

Time integration is by a leapfrog semi-implicit scheme with an Asselin (1972) frequency filter. A time step of 12 minutes is used for dynamics and physics, except for full calculation of atmospheric radiation, which is done hourly for the shortwave fluxes and every 3 hours for the longwave fluxes. An implicit scheme with explicit coefficients also is used to eliminate numerical oscillation while integrating the coupled heat and mass exchanges between the surface and the atmospheric boundary layer (cf. Sato et al. 1989a). 


\section{COLA}

\section{Smoothing / Filling}

Mean silhouette orography is determined for each Gaussian grid box (see Orography). Negative atmospheric moisture values arising from the model's spectral truncation are filled by resetting these to zero.

\section{Sampling Frequency}

For the AMIP simulation, the model history is written every 6 hours.

\section{Dynamical/Physical Properties}

\section{Atmospheric Dynamics}

Primitive-equation dynamics are expressed in terms of vorticity, divergence, surface pressure, specific humidity, and virtual temperature.

\section{Diffusion}

- Fourth-order $\left(\nabla^{4}\right)$ horizontal diffusion is applied to the vorticity, divergence, specific humidity, and virtual temperature. (The coefficient of diffusion for the divergence is $0.61 \times 10^{16} \mathrm{~m}^{4} \mathrm{~s}^{-1}$, while it is $0.81 \times 10^{16} \mathrm{~m}^{4} \mathrm{~s}^{-1}$ for the other fields.) For the specific humidity and virtual temperature, the $\nabla^{2}$ correction necessary to account for diffusion on constant pressure (rather than constant sigma) surfaces is also applied. This correction includes a priori specification of estimates for global-mean specific humidity and temperature.

- Stability-dependent vertical diffusion with Mellor and Yamada (1982) level-2 turbulence closure is used in the planetary boundary layer and free atmosphere. To obtain the eddy diffusion coefficients, a prognostic equation is solved for the turbulent kinetic energy, with other second-order moments being calculated diagnostically.

\section{Gravity-wave Drag}

Gravity-wave drag is simulated as described by Kirtman et al. (1993) and Alpert et al. (1988). The parameterization includes determination of the momentum flux due to gravity waves at the surface, as well as at higher levels. The gravity-wave drag (stress) is given by the convergence of the vertical momentum flux. The surface stress 
is calculated as a nonlinear function of both the surface wind speed and the local Froude number, following Pierrehumbert (1987). Vertical variations in the momentum flux occur when the local value of the wave-modified Richardson number becomes less than 0.25 and the stress vanishes--cf. Eliassen and Palm 1961), or when wave breaking occurs (the local Froude number becomes critical); in the latter case the momentum flux is reduced according to the wave saturation hypothesis of Lindzen (1981). See also Orography.

\section{Solar Constant/Cycles}

The solar constant is the AMIP-prescribed value of $1365 \mathrm{~W} \mathrm{~m}^{-2}$. Both seasonal and diurnal cycles in solar forcing are simulated.

\section{Chemistry}

The carbon dioxide concentration is the AMIP-prescribed value of $345 \mathrm{ppm}$. Seasonal zonal profiles of ozone are prescribed from GFDL climatologies, with daily values obtained by linear interpolation. Radiative effects of water vapor, but not those of aerosols, also are included (see Radiation).

\section{Radiation}

- The radiation code follows Harshvardhan et al. (1987). The shortwave scheme is based on the method of Lacis and Hansen (1974). Six absorption bands are considered, one for ozone in the ultraviolet (wavelengths $<0.35$ micron) and visible (wavelengths 0.50 to 0.70 micron) spectral ranges, and five near-infrared bands (wavelengths 0.70 to 4.0 microns). At the surface, solar radiative fluxes are separated into four components: the ultraviolet and visible direct and diffuse beams, and the near-infrared direct and diffuse beams (see Surface Characteristics). For clear-sky conditions, a combined surface-atmosphere treatment is employed for Rayleigh scattering. For cloudy skies, multiple scattering effects are treated by a delta-Eddington approach (cf. Joseph et al. 1976). In this case optical depth is estimated from cloud temperature and pressure thickness after Harshvardhan et al. (1989), while the asymmetry factor and single-scattering albedo are prescribed.

- The longwave scheme follows the broadband transmission approach of Chou (1984) for water vapor, that of Chou and Peng (1983) for carbon dioxide, and that of Rodgers (1968) 


\section{COLA}

for ozone. The treatment of continuum absorption by water vapor follows Roberts et al. (1976). Water vapor absorption is calculated in spectral domains corresponding to two band centers $\left(0-3.4 \times 10^{4} \mathrm{~m}^{-1}\right.$ and $\left.1.38 \times 10^{5}-1.90 \times 10^{5} \mathrm{~m}^{-1}\right)$ and the associated band wings (in the range $3.40 \times 10^{4}-3.00 \times 10^{5} \mathrm{~m}^{-1}$ ). Carbon dioxide absorption is treated similarly (with band-center domain $6.80 \times 10^{4}-7.20 \times 10^{4} \mathrm{~m}^{-1}$ and band-wing domains in the range $5.40 \times 10^{4}-8.00 \times 10^{4} \mathrm{~m}^{-1}$ ). Ozone absorption is calculated in the interval $9.80 \times 10^{4}-1.10 \times 10^{5} \mathrm{~m}^{-1}$. For cloudy-sky conditions, longwave emissivity is a function of the optical thickness of the cloud layer (see above). Cumuloform clouds are treated as fully overlapped in the vertical, and stratiform clouds as randomly overlapped (see Cloud Formation).

\section{Convection}

-Penetrative convection is simulated following Kuo (1965) with modifications as described by Sela (1980). Convection occurs in the presence of large-scale moisture convergence accompanied by a moist unstable lapse rate under moderately high relative humidity conditions. The vertical integral of the moisture convergence determines the total moisture available for moistening vs heating (through precipitation formation) the environment. If the moisture convergence in the first several lowest layers of a vertical column exceeds a critical threshold $\left(2 \times 10^{-8} \mathrm{sec}^{-1}\right)$, a moist adiabat is computed assuming the bottom layer is saturated, and using the preliminary pressure and temperature prediction of the model.

- An unstable subcolumn is then defined which extends from the bottom layer to the first layer for which a moist adiabatically lifted air parcel is not warmer than the environment. Within this subcolumn, the departures of the temperature and specific humidity of a saturated parcel from the respective environmental profiles in each layer determine the fraction of the total available moisture contributed to latent heat release vs moistening of that layer; the temperature and humidity profiles are revised accordingly. In addition, if the revised temperature profile exceeds a dry adiabatic lapse rate, a dry convective adjustment is performed and the moisture in the column is vertically redistributed to reflect the adjusted temperature profile. Cf. Sela (1980) for further details.

- Following Tiedtke (1983), simulation of shallow (nonprecipitating) convection is parameterized as an extension of the vertical diffusion scheme (see Diffusion). 


\section{Cloud Formation}

- Cloud formation is simulated following the diagnostic method of Slingo (1987). Two basic cloud types--cumuloform and stratiform--are represented. The height of cumuloform cloud is determined by the level of nonbuoyancy for moist adiabatic ascent in the model's convective scheme (see Convection). The cumuloform cloud fraction (not exceeding 0.8 ) is estimated from the scaled 3-hour mean convective precipitation rate (see Precipitation). In the case of convection penetrating above the $400 \mathrm{hPa}$ level, the cumuloform cloud is capped by a cirrus anvil.

- Up to three separated layers of stratiform cloud are allowed in predefined domains (high, middle, and low). Clouds associated with fronts/tropical disturbances have fractional extent determined by a quadratic function of the difference between the local relative humidity and a threshold value of 80 percent. In the low-cloud domain, the fractional cloudiness is reduced in regions of moist subsidence, while stratus cloud forms if a temperature inversion is present under dry layers.

\section{Precipitation}

- Precipitation is produced both from large-scale condensation and from the convective scheme (see Convection). The large-scale precipitation algorithm compares the predicted specific humidity with a modified saturation value that is a function of temperature and pressure of a vertical layer (cf. Sela 1980). If the predicted humidity exceeds this threshold value, condensation occurs and the predicted temperature field is adjusted to account for the associated latent heat release.

- To prevent convective precipitation when the environment is unstable but relatively dry, the falling condensed water evaporates as it acts to progressively saturate lower layers. Large-scale precipitation may similarly evaporate. In both cases all precipitation that penetrates the bottom atmospheric layer is allowed to fall to the surface. See also Snow Cover.

\section{Planetary Boundary Layer}

The PBL is typically represented by the first six vertical levels, which correspond to pressures of $995,981,960,920,857$, and $779 \mathrm{hPa}$ for a surface pressure of $1000 \mathrm{hPa}$. See also Diffusion, Surface Characteristics, and Surface Fluxes. 


\section{COLA}

\section{Orography}

Silhouette orography is derived from the U.S. Navy terrain height data at a resolution of 10-minute arc (cf. Joseph 1980). These data are scanned to obtain the maximum height for each longitude and latitude encompassed by each model Gaussian grid box. The mean of the combined sets of maxima is then assigned as the silhouette height for each grid box.

\section{Ocean}

AMIP monthly sea surface temperature fields are prescribed, with daily values determined by linear interpolation.

\section{Sea Ice}

AMIP monthly sea ice extents are prescribed. Sea ice covers an entire Gaussian grid box with a fixed thickness of $2 \mathrm{~m}$, and the temperature below the ice is assumed to be $271.16 \mathrm{~K}$. Snow does not accumulate on sea ice. The surface sensible and latent heat fluxes are determined from an energy balance calculation that includes heat conduction through the sea ice (see Surface Fluxes).

\section{Snow Cover}

If the temperature at the lowest atmospheric level is less than $271.16 \mathrm{~K}$, precipitation falls as snow, but its accumulation is accounted for only on land. Snow cover affects both the surface albedo and the heat transfer/capacity of the soil. For purposes of calculating the surface albedo, the fractional coverage of a grid box by snow is a function of its depth and of the maximum snow cover for a grid box. Snow mass is determined prognostically from a budget equation that accounts for accumulation and melting. Snowmelt contributes to soil moisture, and sublimation of snow to the surface evaporation. See also Surface Characteristics, Surface Fluxes, and Land Surface Processes.

\section{Surface Characteristics}

- Roughness lengths over oceans are determined from the surface wind stress after the method of Charnock (1955). The roughness length over sea ice is a uniform $1 \times 10^{-4} \mathrm{~m}$. Over land, the 12 vegetation/surface types of the Simple Biosphere (SiB) 
model of Sellers et al. (1986) and associated monthly varying roughness lengths are specified from data of Dorman and Sellers (1989).

- Over oceans the surface albedo depends on zenith angle, but not spectral interval (cf. Payne 1972). The albedo of sea ice is a constant 0.50. Surface albedos of vegetated land are prescribed after data of Dorman and Sellers (1989), and vary monthly according to seasonal changes in vegetation. The land albedo is specified separately for visible (0.0-0.70 micron) and near-infrared (0.70-4.0 microns) spectral intervals, and is also a function of solar zenith angle. The changes in land albedo associated with partial snow cover (including effects of multiple reflections between snow and the vegetation canopy) are parameterized as described by Xue et al. (1991).

- Surface longwave emissivity is everywhere prescribed to be unity (blackbody emission).

\section{Surface Fluxes}

- Surface solar absorption is determined from the surface albedos, and longwave emission from the Planck equation with uniform emissivity of 1.0 (see Surface Characteristics).

- In the lowest atmospheric layer, surface turbulent eddy fluxes of momentum, heat, and moisture follow Monin-Obukhov similarity theory. To avoid an iterative solution for the surface fluxes, the associated drag and transfer coefficients are approximated as analytical functions of the surface characteristics and bulk Richardson number. Over the oceans, the equations formulated by Miyakoda and Sirutis (1986), expressed as bulk formulae, are used to compute surface fluxes. Over land, stability-dependent drag and transfer coefficients (expressed as aerodynamic and surface resistances) are determined after Xue et al. (1991).

- Surface evaporation is at the potential rate over oceans, snow, and ice. Over land, the surface moisture flux includes both evapotranspiration via vegetation root uptake (including the effects of bulk stomatal resistance) and direct evaporation from the vegetation canopy and from bare soil (see Land Surface Processes).

- Above the constant-flux surface layer, diffusion of momentum, heat, and moisture are predicted by the Mellor and Yamada (1982) level-2 turbulence closure scheme (see Diffusion). 
COLA

\section{Land Surface Processes}

- Land-surface processes are simulated following the Xue et al. (1991) modification of the $\mathrm{SiB}$ model of Sellers et al. (1986). Within the single-story vegetation canopy, evapotranspiration from dry leaves includes detailed modeling of stomatal and canopy resistances; direct evaporation from the wet canopy and from bare soil is also treated (see Surface Fluxes). Precipitation interception by the canopy is simulated, and its infiltration into the ground is limited to less than the hydraulic conductivity of the soil.

- Soil temperature is determined in two layers by the force-restore method of Deardorff (1978). Soil moisture, which is predicted from diffusion equations in three layers, is increased by infiltrated precipitation and snowmelt, and is depleted by evapotranspiration and direct evaporation. Both surface runoff and deep runoff from gravitational drainage are simulated. See also Surface Characteristics and Surface Fluxes. 


\section{CSIRO: Commonwealth Scientific and Industrial Research Organization}

\section{AMIP Representative(s)}

Dr. Barrie Hunt, CSIRO Division of Atmospheric Research, PMB1, Mordialloc, Victoria 3195, Australia; Phone: +61-3-586-7680; Fax: +61-3-586-7600;

e-mail: bgh@dar.csiro.au

Model Designation

CSIRO CSIRO9 Mark 1 R21L9

\section{Model Lineage}

The CSIRO model is derived from earlier two-level and four-level spectral models based on the primitive equations expressed in conservative flux form (cf. Gordon 1981,1993).

\section{Model Documentation}

Documentation of the present version of the CSIRO model is provided by McGregor et al. (1993).

\section{Numerical/Computational Properties}

\section{Horizontal Representation}

Spectral (spherical harmonic basis functions) with transformation to a Gaussian grid for calculation of nonlinear quantities and some physics. The atmospheric moisture field is represented only in gridded form.

\section{Horizontal Resolution}

Spectral rhomboidal 21 (R21), roughly equivalent to $3.2 \times 5.6$ degrees latitudelongitude.

\section{Vertical Domain}

Surface to about $21 \mathrm{hPa}$. For a surface pressure of $1000 \mathrm{hPa}$, the lowest atmospheric level is at about $979 \mathrm{hPa}$. 


\section{CSIRO}

\section{Vertical Representation}

Finite-difference sigma coordinates.

\section{Vertical Resolution}

There are 9 unevenly spaced sigma levels. For a surface pressure of $1000 \mathrm{hPa}$, 3 levels are below $800 \mathrm{hPa}$ and 3 levels are above $200 \mathrm{hPa}$.

\section{Computer/Operating System}

The AMIP simulation was run on a Cray Y/MP computer using one processor in the UNICOS environment.

\section{Computational Performance}

For the AMIP experiment, about 35 seconds Cray Y/MP computation time per simulated day.

\section{Initialization}

For the AMIP simulation, initialization of the atmosphere, soil moisture, and snow cover/depth for 1 January 1979 is from an earlier model simulation with climatological sea surface temperatures.

\section{Time Integration Scheme(s)}

A semi-implicit leapfrog time scheme with an Asselin (1972) frequency filter is used for most calculations, with the momentum surface flux and vertical diffusion above the surface computed by split backward implicit integration. A time step of 30 minutes is used for dynamics and physics, except for full calculations of all radiative fluxes and heating rates, which are done every 2 hours.

\section{Smoothing / Filling}

Orography is truncated at the $\mathrm{R} 21$ resolution of the model (see Orography). To counter the negative values of atmospheric moisture that may otherwise develop, vertical transport of moisture is inhibited if the local water vapor mixing ratio drops below $2 \times 10^{-6} \mathrm{~kg}$ (water) per $\mathrm{kg}$ (air). In addition, negative moisture values are 
removed by a proportional adjustment method while conserving the global mean (cf. Royer 1986). Cf. McGregor et al. 1993 for further details.

\section{Sampling Frequency}

For the AMIP simulation, the model history is written every 6 hours.

\section{Dynamical/Physical Properties}

\section{Atmospheric Dynamics}

Primitive-equation dynamics are expressed in conservative flux form (i.e., weighting vorticity, divergence, temperature, and specific humidity by the prognostic surface pressure) as described by Gordon (1981). Effects of frictional heating are included in the temperature tendency equation, and virtual temperature is used to compute geopotential height.

\section{Diffusion}

- Linear second-order $\left(\nabla^{2}\right)$ horizontal diffusion of the temperature, vorticity, divergence, and moisture fields is computed via a split implicit time integration. Diffusion (with coefficient $10^{6} \mathrm{~m}^{2} \mathrm{~s}^{-1}$ ) is applied to the upper-half of the rhomboid for the spectral temperature, vorticity, and divergence. Diffusion of the gridded moisture is calculated from a temporary spectral representation of the moisture field without surface pressure weighting, and is applied to the entire rhomboid with the diffusion coefficient halved. Diffusion for temperature and moisture contains a first-order correction to constant pressure surfaces.

- Vertical diffusion of momentum, heat, and moisture is parameterized in terms of stability-dependent K-theory following Blackadar (1962), with the choice of asymptotic mixing length after Louis (1979). The calculation of vertical momentum diffusion is via backward implicit time differencing (see Time Integration Schemes).

\section{Gravity-wave Drag}

Under conditions of vertical stability, orographic gravity-wave drag is simulated after the method of Chouinard et al. (1986). The drag at the surface is dependent on sub-gridscale orographic variance (see Orography), and it is parameterized by means 


\section{CSIRO}

of a "launching" height which is defined to be twice the local standard deviation of the surface heights. Following Palmer et al. (1986), the maximum launching height is limited to $800 \mathrm{~m}$ in order to prevent two-grid noise near steep mountains. At a particular sigma level the frictional drag on the atmosphere from breaking gravity waves depends on the projection of the wind on the surface wind and on the Froude number, which in turn is a function of the launching height, the atmospheric density, the Brunt-Vaisalla frequency, and the wind shear. Gravity-wave drag is assumed to be zero above a critical level, which is taken to be the top sigma level of the model (see Vertical Domain).

\section{Solar Constant/Cycles}

The solar constant is the AMIP-prescribed value of $1365 \mathrm{~W} \mathrm{~m}^{-2}$. Both seasonal and diurnal cycles in solar forcing are simulated.

\section{Chemistry}

The carbon dioxide concentration is the AMIP-prescribed value of $345 \mathrm{ppm}$. Ozone concentrations, specified as a function of latitude and pressure, are interpolated from the Dopplick (1974) seasonal climatology. Radiative effects of water vapor, but not of aerosols, also are included (see Radiation).

\section{Radiation}

- The radiation code is after Fels (1985), Fels and Schwarzkopf $(1975,1981)$, and Schwarzkopf and Fels (1991). The shortwave calculations are based on a modified Lacis and Hansen (1974) approach. The shortwave spectrum is divided into 9 bands, the first band covering the ultraviolet (wavelengths 0.1 to 0.4 micron) and visible ( 0.4 to 0.7 micron) spectral intervals, while the other 8 bands are in the near-infrared ( 0.7 to 4.0 microns). Rayleigh scattering by air molecules and absorption by ozone and water vapor are treated in the first band. In the 8 nearinfrared bands, variable absorption by water vapor is included, and carbon dioxide absorption is calculated after a modified Sasamori et al. (1972) method. Pressure corrections and multiple reflections between clouds and the surface are treated, but not the radiative effects of aerosols. 
- Longwave calculations follow the simplified exchange method of Fels and Schwarzkopf (1975) and Schwarzkopf and Fels (1991) applied over seven spectral bands (with wavenumber boundaries at $0,4.0 \times 10^{4}, 5.6 \times 10^{4}, 8.0 \times 10^{4}, 9.9 \times 10^{4}, 1.07 \times 10^{5}$, $1.20 \times 10^{5}$, and $2.20 \times 10^{5} \mathrm{~m}^{-1}$ ). Absorption by the vibrational and rotational lines of water vapor, carbon dioxide, and ozone, as well as continuum absorption by water vapor are treated, but some weak absorption bands of ozone and carbon dioxide are neglected. Carbon dioxide transmission coefficients are calculated for the actual temperature and pressure profile of each vertical column after the interpolation method of Fels and Schwarzkopf (1981). Longwave ozone and water vapor absorption (including temperature effects) are computed by a random-band model.

- Cloud optical properties are prescribed. In the visible, cloud absorptivity is assumed to be zero; infrared absorptivity depends on cloud height, as do the visible and infrared cloud reflectivities. The absorptivity and reflectivity of clouds are also proportional to cloud amount. Longwave emissivity is prescribed as unity (blackbody emission) for all clouds. For purposes of calculating radiation and top-of-atmosphere cloud cover, clouds in different vertical layers are assumed to be randomly overlapped. Cf. McGregor et al. (1993) for further details. See also Cloud Formation.

\section{Convection}

- After checking for supersaturation and attendant release of precipitation (see Precipitation) and performing a dry convective adjustment if needed, a modified Arakawa (1972) "soft" moist adjustment scheme predicts any subsequent precipitation release and the redistribution of moisture and momentum that may occur within sub-gridscale cumulus towers. These form when a layer (other than the lowest layer) is moist unstable with respect to at least one layer above, and when the relative humidity in the lowest unstable layer is $>75$ percent. It is assumed that a constant convective mass flux effects a vertical redistribution of heat within the cumulus tower, such that the moist instability at each level (the difference between the moist static energy at cloud base and the saturation value at each level) decays with an e-folding time of one hour. (The heating at cloud base is assumed to be zero to ensure closure for the convective scheme.)

- The attendant moisture redistribution and removal (see Precipitation) results in drying of the environment if the ambient relative humidity is at least 60 percent. 


\section{CSIRO}

The convective mass flux also transfers momentum upward through the cumulus tower, and downward via the surrounding large-scale descent.

- Shallow cumulus convection is parameterized by a modified Geleyn (1987) scheme that operates as an extension of the vertical diffusion of heat and momentum. The stability dependence of this diffusion is defined by a modified moist bulk Richardson number. Cf. McGregor et al. (1993) for further details.

\section{Cloud Formation}

- If convective activity occurs in the previous timestep (see Convection), fixed convective cloud fractions are set according to height ( 0.55 for low cloud, 0.42 for middle cloud, and 0.25 for high cloud). For each height class, convective cloud is confined to single layers.

- Large-scale cloud amounts are determined from a modified form of the Rikus (1991) diagnostic, which is a quadratic function of the difference between the relative humidity of a layer and critical humidities that depend on cloud height (low, middle, and high cloud). Following Saito and Baba (1988), maximum cloud fractions are also specified for each cloud type ( 0.70 for low cloud, 0.53 for middle cloud, and 0.50 for high cloud). Middle and high clouds are restricted to single layers, while low clouds can occupy two layers.

- Stability-dependent low cloud associated with temperature inversions also may form if the relative humidity at cloud base is at least 60 percent. The cloud fraction is a function of the intensity of the temperature inversion, following Slingo (1987). The overall fraction of low cloud is then set to the largest value predicted by either this stabilitydependent diagnostic or by another operative mechanism $(0.55$ in the case of convective activity, or 0.70 for large-scale condensation).

\section{Precipitation}

Precipitation forms as a result of supersaturation and/or the moist convective adjustment process (see Corvection). There is no subsequent evaporation of precipitation.

\section{Planetary Boundary Layer}

There are typically two atmospheric levels in the model PBL (whose top is not explicitly determined, however). In order to validate the simulation of surface atmospheric temperature against observations, a 2-meter (screen height) 
temperature is calculated from the bulk Richardson number determined for the lowest model layer (by applying the Monin-Obukhov assumption of constant momentum and heat fluxes in the surface layer). For unstable conditions, this requires an iterative solution. For purposes of computing surface fluxes, however, atmospheric winds, temperatures, and humidities at the first full atmospheric level above the surface (at sigma $=0.979$ ) are used (see Surface Fluxes). Cf. McGregor et al. (1993).

\section{Orography}

Orography from the $1 \times 1$-degree data of Gates and Nelson (1975) is transformed to spectral coefficients and truncated at the R21 resolution of the model. Orographic variance data (supplied by the United Kingdom Meteorological Office) are also used for the parameterization of gravity-wave drag (see Gravity-wave Drag).

\section{Ocean}

AMIP monthly sea surface temperature fields are prescribed, with daily values determined by linear interpolation.

\section{Sea Ice}

Monthly AMIP sea ice extents are prescribed, with thicknesses specified to be a uniform $2 \mathrm{~m}$. The ice surface temperature is predicted from the net flux of energy into the surface layer (see Surface Fluxes), which includes conduction heating that is proportional to the difference between the ice surface temperature and that prescribed $(271.5 \mathrm{~K})$ for the ocean below. A flux of $2 \mathrm{~W} \mathrm{~m}^{-2}$ is also directed into the ice from below to represent the lateral convergence of heat transport by the underlying ocean. Snow may accumulate on sea ice, and sublimation and melting may reduce this snow cover (see Snow Cover).

\section{Snow Cover}

Precipitation falls to the surface as snow if the temperature of the second atmospheric vertical level above the surface (at sigma $=0.914$ ) is below $0{ }^{\circ} \mathrm{C}$. The latent heat is incorporated into the surface temperature prognostic for non-ocean surfaces (see Sea Ice and Land Surface Processes). Prognostic snow mass, with accumulation and melting over both land and sea ice, is included, but the allowable 


\section{CSIRO}

snow depth is limited to $4 \mathrm{~m}$. Snow cover affects the heat capacity and conductivity of the land surface (see Land Surface Processes), and sublimation of snow contributes to the surface evaporative flux (see Surface Fluxes). Melting of snow, which contributes to soil moisture, occurs when the surface (top soil layer or sea ice) temperature is $>0{ }^{\circ} \mathrm{C}$. Snow cover affects the albedo of the surface, but with less impact if the snow is melting (see Surface Characteristics).

\section{Surface Characteristics}

- The roughness length over land is everywhere prescribed to be $0.17 \mathrm{~m}$ for calculation of surface momentum fluxes, and $0.023 \mathrm{~m}$ for surface heat and moisture fluxes (see Surface Fluxes). However, the roughness length for other surface types is the same for heat and momentum fluxes: over ice, the roughness is a uniform $0.001 \mathrm{~m}$, while over the ocean it is a function of surface wind stress, following Charnock (1955).

- The albedo of sea ice is a constant 0.65. Over oceans, zenith-angle dependent surface albedos (minimum 0.04, maximum 0.33) are computed after the method of Washington and Meehl (1984) for a single spectral interval. Over land, the mean annual background albedos of Posey and Clapp (1964) are modified by snow cover: an albedo of 0.80 is assumed for snow $>0.10 \mathrm{~m}$ in depth, but is set to 0.50 for melting snow. For snow depths $<0.10 \mathrm{~m}$, the albedo is interpolated between snow-free and snow-covered values as a nonlinear function of the snow depth.

- Longwave emissivity is set to unity (blackbody emission) for all surface types. Cf. McGregor et al. (1993) for further details.

\section{Surface Fluxes}

- Surface solar absorption is determined from surface albedos, and surface longwave emission from the Planck equation with prescribed emissivity of 1.0 (see Surface Characteristics).

- Following Monin-Obukhov similarity theory applicable to a constant-flux layer, surface turbulent eddy fluxes are expressed as bulk formulae. The requisite atmospheric surface winds, potential temperatures, and specific humidities are taken to be those at the first vertical level above the surface (at sigma $=0.979$ ). The effective ground value of specific humidity needed for determination of the surface moisture flux from the bulk formula is obtained as a fraction $\alpha$ of the saturated humidity at the 
ground temperature, where $\alpha$ is a function of soil moisture (see Land Surface Processes) or is prescribed to be 1 over oceans and ice.

- The bulk drag and transfer coefficients are functions of roughness length (see Surface Characteristics) and vertical stability (bulk Richardson number) following Louis (1979), with the same transfer coefficient used for the heat and moisture fluxes. Over the oceans, the neutral transfer coefficient for surface heat and moisture fluxes is a constant $8.5 \times 10^{-4}$.

\section{Land Surface Processes}

- Soil temperature is computed by modeling heat diffusion in three layers, with thicknesses $(0.03,0.26$ and $2.5 \mathrm{~m}$ ) chosen to represent both diurnal and seasonal temperature fluctuations. In the case of snow cover (see Snow Cover), the thickness of the top layer is $0.23 \mathrm{~m}$, and values of density, specific heat, and thermal diffusivity are modified. The lower boundary condition is zero net heat flux, while the upper boundary condition is a net balance of all the surface heat fluxes (see Surface Fluxes).

- Soil moisture is modeled by the force-restore method of Deardorff (1977), with a time constant of 1 day. There is no explicit modeling of the effects of vegetation. Soil moisture is computed in two layers: a thin surface layer $0.005 \mathrm{~m}$ thick to capture diurnal variations, and an underlying reservoir $0.50 \mathrm{~m}$ thick for longer-term variations. Saturation values of soil moisture are $0.16 \mathrm{~m}$ (the field capacity) in the lower layer and $0.0018 \mathrm{~m}$ in the upper layer. For snow-covered surfaces, the soil moisture in the upper layer is a fraction of the saturated value that is given by an empirical function of the snow temperature. Both precipitation and snowmelt contribute to soil moisture, while evaporation depletes it. The fraction $\alpha$ of ground saturation humidity that is available for evaporation (see Surface Fluxes) is given by the ratio of soil moisture in the upper layer to its saturation value. Runoff occurs if soil moisture exceeds field capacity $(0.16 \mathrm{~m})$ or if the precipitation rate at any timestep is greater than an equivalent rate of $0.015 \mathrm{~m} \mathrm{day}^{-1}$. Cf. McGregor et al. (1993) for further details. 


\section{CSU: Colorado State University}

\section{AMIP Representative(s)}

Dr. David Randall, Atmospheric Sciences Department, Colorado State University, Fort Collins, Colorado 80523; Phone: +1-303-491-8474; Fax: +1-303-491-8449; e-mail: randall@redfish.ATMOS.ColoState.EDU.

Model Designation

CSU CSU91 4x5L17

\section{Model Lineage}

The CSU model is derived from an earlier version of the University of Califronia at Los Angeles (UCLA) atmospheric general circulation model. Subsequent modifications principally include changed parameterizations of radiation, cloud optical properties, land surface processes, and model diagnostics.

\section{Model Documentation}

Key documents on CSU model features and applications include those by Randall (1987, 1989), and Randall et al. (1985, 1989, 1990). Details of the treatment of the planetary boundary layer (PBL) are given by Suarez et al. (1983). The atmospheric radiation schemes are described by Harshvardhan et al. $(1987,1989)$ and Stephens et al. (1993).

\section{Numerical/Computational Properties}

\section{Horizontal Representation}

Finite differences on a C-grid (cf. Arakawa and Lamb 1977), conserving total atmospheric mass, energy, and potential enstrophy. The horizontal differencing scheme is of second-order accuracy, except that the inertial terms of the momentum equation correspond to a fourth-order scheme for the advection of vorticity (cf. Takano and Wurtele 1982), and the horizontal advection of potential temperature and of moisture is also of fourth-order accuracy. 


\section{Horizontal Resolution}

$4 \times 5$-degree latitude-longitude grid.

\section{Vertical Domain}

Surface to $51.3 \mathrm{hPa}$. The lowest atmospheric level is identically the top of the prognostic PBL (see Planetary Boundary Layer), which nominally varies up to $180 \mathrm{hPa}$ above the surface.

\section{Vertical Representation}

Finite differences in modified sigma coordinates. For $\mathrm{P}$ the pressure at a given level, $\mathrm{P}_{\mathrm{T}}=51.3 \mathrm{hPa}$ the constant pressure at the model top, $\mathrm{P}_{\mathrm{I}}=100 \mathrm{hPa}$ the pressure at the tropopause, $\mathrm{P}_{\mathrm{B}}$ the pressure at the top of the prognostic PBL (see Planetary Boundary Layer $)$, and $\mathrm{P}_{\mathrm{S}}$ the pressure at the earth's surface, sigma $=\left(\mathrm{P}-\mathrm{P}_{\mathrm{I}}\right) /\left(\mathrm{P}_{\mathrm{I}}-\mathrm{P}_{\mathrm{T}}\right)$ for $\mathrm{P}_{\mathrm{I}} \geq \mathrm{P} \geq \mathrm{P}_{\mathrm{T}}$ (in the stratosphere); sigma $=\left(\mathrm{P}_{\mathrm{B}}-\mathrm{P}\right) /\left(\mathrm{P}_{\mathrm{B}}-\mathrm{P}_{\mathrm{I}}\right)$ for $\mathrm{P}_{\mathrm{B}} \geq \mathrm{P} \geq \mathrm{P}_{\mathrm{I}}$ (in the troposphere above the $\mathrm{PBL})$; and sigma $=1+\left(\mathrm{P}_{\mathrm{S}}-\mathrm{P}\right) /\left(\mathrm{P}_{\mathrm{S}}-\mathrm{P}_{\mathrm{B}}\right)$ for $\mathrm{P}_{\mathrm{S}} \geq \mathrm{P} \geq \mathrm{P}_{\mathrm{B}}$ (in the PBL). Following Tokioka (1978), the sigma levels in the model stratosphere are evenly spaced in the logarithm of pressure. Cf. Randall (1989) for further details.

\section{Vertical Resolution}

There are 17 unevenly spaced modified sigma levels (see Vertical Representation). The first layer is identically the model's prognostic PBL of varying depth (see Planetary Boundary Layer). For a surface pressure of $1000 \mathrm{hPa}, 2$ levels are typically below $800 \mathrm{hPa}$ (depending on the PBL depth) and 6 levels are typically above $200 \mathrm{hPa}$.

\section{Computer/Operating System}

The AMIP simulation was run on a Cray 2 computer using 1 processor in a CTSS environment.

\section{Computational Performance}

For the AMIP experiment, about 6.5 minutes Cray 2 computation time per simulated day. 


\section{Initialization}

For the AMIP simulation, the model atmosphere and snow cover/depth are initialized for 1 January 1979 from a previous model solution; soil moisture is initialized from the January climatological estimates of Mintz and Serafini (1981).

\section{Time Integration Scheme(s)}

Adiabatic, frictionless processes (advection, pressure gradient force, etc.) employ the leapfrog scheme with a time step of 6 minutes. A 6-minute Matsuno step is inserted at 1-hour intervals to prevent separation of the solutions from the presence of a computational mode; prior to each of these the heating, moistening, and cumulus mass fluxes are computed and added evenly over the succeeding Matsuno and leapfrog steps. Radiation is calculated at 1-hour intervals. The surface fluxes and temperatures are computed implicitly, and the horizontal diffusion of momentum is calculated by a forward time differencing scheme, both with time steps of 6 minutes.

\section{Smoothing / Filling}

Orography is smoothed (see Orography). The mass flux and pressure gradient vectors are Fourier filtered to maintain computational stability near the poles (cf. Arakawa and Lamb 1977). Spurious negative values of atmospheric specific humidity are filled by redistributing moisture, without changing its global integral. This correction is implemented by a global multiplicative hole-filler (cf. Rood 1987) that "borrows" moisture primarily from grid boxes where it is plentiful--e.g., from within the PBL (see Planetary Boundary Layer).

\section{Sampling Frequency}

For the AMIP simulation, the model history is written every 6 hours.

\section{Dynamical/Physical Properties}

\section{Atmospheric Dynamics}

Primitive-equation dynamics are expressed in terms of atmospheric potential temperature, u-v winds, specific humidity, and surface pressure. The depth and turbulence kinetic energy (TKE) of the PBL are also prognostic variables (see Planetary Boundary Layer). 
Diffusion

- Nonlinear second-order horizontal diffusion after Smagorinsky (1963) is applied only to the momentum equation at all vertical levels.

- Vertical diffusion is not modeled above the PBL (see Planetary Boundary Layer).

Gravity-wave Drag

Gravity-wave drag is not modeled.

\section{Solar Constant/Cycles}

The solar constant is the AMIP-prescribed value of $1365 \mathrm{~W} \mathrm{~m}^{-2}$. Both seasonal and diurnal cycles in solar forcing are simulated.

\section{Chemistry}

The carbon dioxide concentration is the AMIP-prescribed value of $345 \mathrm{ppm}$. Monthly average zonal profiles of ozone are prescribed from data of McPeters et al. (1984). Radiative effects of water vapor are also included, but not those of aerosols (see Radiation).

\section{Radiation}

- Atmospheric radiation is after the method of Harshvardhan et al. $(1987,1989)$. The shortwave parameterization by Davies (1982) follows the approach of Lacis and Hansen (1974) for clear-sky Rayleigh scattering, for ozone absorption in the ultraviolet (wavelengths $<0.35$ micron) and visible (0.5-0.7 micron) spectral bands, and for water vapor absorption in the near-infrared (0.7-4.0 microns).

- For longwave absorption, the broadband approach of Chou (1984) is used for water vapor, that of Chou and Peng (1983) for carbon dioxide, and that of Rodgers (1968) for ozone. Longwave fluxes are calculated in five spectral bands (wavenumbers 0 to $3 \times 10^{5} \mathrm{~m}^{-1}$ ), with continuum absorption by water vapor treated as in Roberts et al. (1976).

- Shortwave absorption/scattering by clouds is modeled by a delta-Eddington approximation, with prescribed single-scattering albedo and asymmetry parameter. The shortwave optical thickness is proportional to the pressure thickness, where the proportionality constant is a function of the mean layer temperature (closely related 


\section{CSU}

to cloud liquid water content). Cloud shortwave scattering is also a function of solar zenith angle. Cloud longwave emissivity depends on a prescribed diffusivity factor and an optical depth which is proportional to that for the shortwave. For purposes of the radiation calculations, all clouds are assumed to be fully overlapped in the vertical See also Cloud Formation.

\section{Convection}

- Penetrative convection is simulated by the Arakawa-Schubert (1974) scheme, as implemented by Lord (1978) and Lord et al. (1982). Convection is assumed to originate in the PBL (see Planetary Boundary Layer), and to carry its mean properties into the free atmosphere. Convective mass fluxes are predicted for 14 mutually interacting cumulus subensembles (cloud types) with different entrainment rates and levels of neutral buoyancy that define the tops of the clouds and their associated convective updrafts. In turn, the predicted convective mass fluxes feed back on the large-scale fields of temperature (through latent heating and compensating subsidence), moisture (through precipitation and detrainment), and momentum (through cumulus friction). The cumulus mass flux is also a mass sink for the PBL, tending to make it more shallow in the absence of compensating large-scale convergence and/or turbulent entrainment of mass. The effects of convective-scale downdrafts are neglected, however.

- The mass flux for each cumulus subensemble is predicted from an integral equation that includes a positive-definite work function (defined by the tendency of cumulus kinetic energy for the subensemble) and a negative-definite kernel which expresses the effects of other subensembles on this work function. The predicted cumulus mass fluxes are positive-definite optimal solutions of this integral equation under the constraint that the rate of generation of conditional convective instability by the large-scale environment is balanced by the rate at which the cumulus subensembles suppress this instability via large-scale feedbacks. Cf. Lord et al. (1982) for further details.

- A moist convective adjustment process after Manabe et al. (1965) simulates midlevel convection originating above the $\mathrm{PBL}$. When the lapse rate exceeds moist adiabatic from one layer to the next and saturation occurs in both layers, mass is mixed such that either the lapse rate is restored to moist adiabatic or saturation is eliminated. The usual outcome is a highly supersaturated upper layer whose excess relative 
humidity is removed by formation of large-scale precipitation (see Precipitation). In addition, if the local lapse rate becomes dry convectively unstable, moisture and enthalpy are redistributed vertically, effectively deepening the PBL beyond its nominal maximum depth of $180 \mathrm{hPa}$ (see Planetary Boundary Layer).

\section{Cloud Formation}

- Clouds of three types form below $100 \mathrm{hPa}$ in the model: optically thick anvil cloud when penetrative convection occurs above $500 \mathrm{hPa}$, large-scale supersaturation cloud when the local relative humidity exceeds 100 percent, and PBL stratocumulus cloud of arbitrary thinness when the prognostic humidity reaches the saturation value and there is a strong inversion at the PBL top (see Planetary Boundary Layer).

- Above the PBL the entire grid box is filled with cloud (i.e., fractional cloudiness of 1 ). The fraction of PBL cloud varies linearly with cloud thickness (ranging between 0 and 1 for a cloud thickness of $12.5 \mathrm{hPa}$ or greater). Cf. Randall et al. (1989) for further details. See also Radiation for cloud-radiative interactions.

\section{Precipitation}

- Precipitation forms when there is large-scale supersaturation and condensation and/or when precipitation is produced by moist convective adjustment (see Convection). Large-scale precipitation can originate in the PBL if the liquid water mixing ratio at the surface is positive (see Planetary Boundary Layer).

- Water/ice that is detrained at the top of convective cloud is assumed to evaporate instantaneously. Precipitation also evaporates as it falls into subsaturated layers, which are moistened and cooled until they become saturated, or until the precipitate is exhausted. There is no evaporation of precipitation in the PBL.

\section{Planetary Boundary Layer}

- The PBL is parameterized as a well-mixed layer (turbulent fluxes vary linearly in the vertical) whose depth changes prognostically as a function of horizontal mass convergence, entrainment, and cumulus mass flux determined from the convective parameterization (see Convection). The PBL is identical to the lowest model layer and its potential temperature, $\mathrm{u}-\mathrm{v}$ winds, and specific humidity are prognostic variables. TKE due to shear production is also predicted from a closure condition involving 


\section{CSU}

dissipation, buoyant consumption, and the rate at which TKE is supplied to make newly entrained air turbulent.

- The presence of PBL stratocumulus cloud (see Cloud Formation) affects the radiative parameterizations (see Radiation), the entrainment rate (through enhanced cloud-top radiative cooling and latent heating), and the exchange of mass with the layer above the PBL as a result of layer cloud instability (LCI). When LCI occurs, the PBL depth remains unchanged. If the PBL lapse rate is dry convectively unstable, an adjustment process is initiated that redistributes moisture and enthalpy vertically to restore stability (see Convection). Cf. Suarez et al. (1983) and Randall et al. (1985), and Randal (1987) for further details. See also Surface Characteristics and Surface Fluxes.

\section{Orography}

The orography is specified from subjectively smoothed data obtained from UCLA.

\section{Ocean}

AMIP monthly sea surface temperature fields are prescribed, with daily values determined by linear interpolation.

\section{Sea Ice}

AMIP monthly sea ice extents are prescribed. The ice thickness is specified to be a uniform $1.5 \mathrm{~m}$ in the Northern Hemisphere, and $1.0 \mathrm{~m}$ in the Southern Hemisphere, except in the month after which seasonal ice first appears or disappears; in these cases, the daily thickness is adjusted linearly between $0 \mathrm{~m}$ and its nominal hemispheric value. The surface temperature of sea ice is determined from the net balance of the surface energy fluxes (see Surface Fluxes) and subsurface heat conduction, which depends on the ice thickness and the temperature of the underlying ocean (fixed at $271.2 \mathrm{~K}$ ). Snow is not allowed to accumulate on sea ice.

\section{Snow Cover}

Precipitation falls as snow if the surface air temperature is $<0^{\circ} \mathrm{C}$. Snow may accumulate only on land, and it is assumed to cover the whole of each grid box where it falls (i.e., snow-cover fraction is unity). The snow depth is predicted from 
a budget equation that includes the rates of snowfall, sublimation, and snowmelt. Sublimation contributes to the total surface evaporation, and snowmelt to soil moisture. Snow cover alters the surface albedo and the heat capacity of the underlying soil. See also Surface Characteristics, Surface Fluxes, and Land Surface Processes.

\section{Surface Characteristics}

- Twelve vegetation types are distinguished and their spatial extents vary monthly.

- The spatially varying roughness lengths over land change monthly, depending on vegetation type. Over ocean, the roughness length is a uniform $2 \times 10^{-4} \mathrm{~m}$, and over ice surfaces, a uniform $1 \times 10^{-4} \mathrm{~m}$.

- Ocean surface albedo depends on solar zenith angle but not on spectral interval. The land albedos vary monthly according to the distinguished vegetation types and are specified for diffuse- and direct-beam components in visible and near-infrared spectral bands. The direct-beam component also depends on solar zenith angle. Land albedo is modified by snow (see Snow Cover). The nominal albedos of snow and sea ice are 0.8 in the visible and 0.4 in the near-infrared; for continental ice, their values are 0.8 and 0.5 , respectively. These albedos are reduced by 40 percent when the temperature of the ice or snow is within $0.05 \mathrm{~K}$ of the melting point.

- Longwave emissivity is prescribed as unity (blackbody emission) for all surfaces.

\section{Surface Fluxes}

- Surface solar absorption is determined from the prescribed albedos, and longwave emission from the Planck equation with emissivity of 1.0 (see Surface Characteristics).

- Turbulent eddy surface fluxes are expressed as bulk formulae following a simplified Deardorff (1972) approach; the drag/transfer coefficients are functions of the PBL height, the surface roughness length, and the vertical stability (bulk Richardson number). The same transfer coefficient is used for the surface fluxes of heat and moisture. The surface wind, temperature, and moisture required for the bulk formulae 
are the prognostic PBL values (see Planetary Boundary Layer). The surface wind speed is constrained to be a minimum of $1 \mathrm{~m} \mathrm{~s}^{-1}$.

- The flux of surface moisture also depends on the evapotranspiration efficiency $\beta$, which is prescribed as 1 over oceans, sea ice, and snow, but which over land is a function of soil moisture (see Land Surface Processes).

Land Surface Processes

- Soil temperature is predicted from heat storage in a single layer, and is solved by an implicit time integration from the net surface energy balance (see Surface Fluxes). The heat capacity of the soil depends on both soil moisture and snow cover.

- Prognostic soil moisture is determined from a single-layer "bucket" model after Manabe (1969) with uniform field capacity of $0.15 \mathrm{~m}$. Precipitation and snowmelt contribute to soil moisture, while surface evaporation depletes it. The evapotranspiration efficiency $\beta$ (see Surface Fluxes) is a function of the ratio of soil moisture to field capacity, with runoff occurring implicitly if this ratio exceeds unity. 


\title{
DERF: Dynamical Extended-Range Forecasting (at Geophysical Fluid Dynamics Laboratory)
}

\begin{abstract}
AMIP Representative(s)
Mr. William Stern and Dr. Kiku Miyakoda, Dynamical Extended-Range Forecasting, Geophysical Fluid Dynamics Laboratory/NOAA, Princeton University, P.O. Box 308, Princeton, New Jersey 08540; Phone: +1-609-452-6545; Fax: +1-609-9875063; e-mail: wfs@GFDL.GOV (Stern)
\end{abstract}

\section{Model Designation}

DERF GFDLSM392.2 T42L18

\section{Model Lineage}

One of several versions of global spectral models in use at the Geophysical Fluid Dynamics Laboratory (GFDL), the DERF model is applied to dynamical extendedrange forecast studies. The DERF model is similar in some respects to the GFDL climate model (e.g., as documented by Manabe and Hahn 1981), but also displays a number of significant differences (e.g., use of triangular rather than rhomboidal spectral truncation and differences in horizontal/vertical resolution and in some physics schemes).

\section{Model Documentation}

Key documentation of model features is given by Gordon and Stern (1982), Gordon (1986, 1992), and Gordon and Hovanec (1985), with additional details on the physics schemes provided by Miyakoda and Sirutis (1977, 1986). Extendedrange forecasting results are summarized by Miyakoda et al. $(1979,1986)$.

\section{Numerical/Computational Properties}

\section{Horizontal Representation}

Spectral (spherical harmonic basis functions) with transformation to a Gaussian grid for calculation of nonlinear quantities and some physics. 


\section{DERF}

\section{Horizontal Resolution}

Spectral triangular 42 (T42), roughly equivalent to $2.8 \times 2.8$ degrees latitudelongitude.

\section{Vertical Domain}

Surface to $2.2 \mathrm{hPa}$. For a surface pressure of $1000 \mathrm{hPa}$, the lowest atmospheric level is at a pressure of about $998 \mathrm{hPa}$.

\section{Vertical Representation}

Finite-difference sigma coordinates.

\section{Vertical Resolution}

There are 18 unevenly spaced sigma levels. For a surface pressure of $1000 \mathrm{hPa}$, 5 levels are below $800 \mathrm{hPa}$ and 5 levels are above $200 \mathrm{hPa}$.

\section{Computer/Operating System}

The AMIP simulation was run on a Cray Y/MP computer using a single processor in a UNICOS operating environment.

\section{Computational Performance}

For the AMIP experiment, about 5.5 minutes Cray Y/MP computation time per simulated day.

\section{Initialization}

For the AMIP simultation, the model atmosphere is initialized for 1 January 1979 from NMC analyses for 22 December 1978, and soil moisture and snow cover/ depth are initialized from ECMWF analyses.

\section{Time Integration Scheme(s)}

A leapfrog semi-implicit scheme similar to that of Bourke (1974) with Asselin (1972) frequency filter is used for time integration. The time step is 15 minutes for dynamics and physics, except for full calculation of all radiative fluxes every 12 hours. 


\section{Smoothing / Filling}

After condensation, filling of negative moisture values (that arise because of spectral truncation) is implemented by borrowing moisture from nearest east-west neighbors, but only if this is sufficient to make up the deficit (cf. Gordon and Stern 1982).

\section{Sampling Frequency}

For the AMIP simulation, the model history is written every 6 hours.

\section{Dynamical/Physical Properties}

\section{Atmospheric Dynamics}

Primitive-equation dynamics are expressed in terms of vorticity, divergence, surface pressure, specific humidity, and temperature (with a linearized correction for virtual temperature in diagnostic quantities, where applicable).

\section{Diffusion}

- Linear fourth-order $\left(\nabla^{4}\right)$ horizontal diffusion is applied to vorticity, divergence, temperature, and specific humidity on constant sigma surfaces.

- Stability-dependent vertical diffusion with Mellor and Yamada (1982) level-2.5 turbulence closure is applied in the planetary boundary layer and free atmosphere. To obtain the eddy diffusion coefficients, a prognostic equation is solved for the turbulence kinetic energy (TKE), with other second-order moments being calculated diagnostically (cf. also Miyakoda and Sirutis 1977).

\section{Gravity-wave Drag}

Gravity-wave drag is simulated after the method of Stern and Pierrehumbert (1988), with wave breaking determining the vertical distribution of momentum flux absorption. Wave breaking occurs when the vertically propagating momentum flux exceeds a saturation flux profile, which is based on criteria for convective overturning.

\section{Solar Constant/Cycles}

The solar constant is the AMIP-prescribed value of $1365 \mathrm{~W} \mathrm{~m}^{-2}$. A seasonal, but not a diurnal cycle in solar forcing, is simulated. 


\section{DERF}

\section{Chemistry}

The carbon dioxide concentration is the AMIP-prescribed value of $345 \mathrm{ppm}$. Zonally averaged seasonal mean ozone distributions are specified from a dataset derived from 1970s balloon-borne ozone-sonde measurements and (above $10 \mathrm{hPa}$ ) on limited satellite and rocket observations. These data are linearly interpolated for intermediate times. Radiative effects of water vapor, but not of aerosols, also are included (see Radiation).

\section{Radiation}

- Shortwave Rayleigh scattering, and absorption in ultraviolet (wavelengths less than 0.35 micron) and visible (0.5-0.7 micron) spectral bands by ozone, and in the nearinfrared (0.7-4.0 microns) by water vapor follows the method of Lacis and Hansen (1974). Pressure corrections and multiple reflections between clouds and the surface are treated. Radiative effects of aerosols are not included.

- Longwave radiation follows the simplified exchange method of Fels and Schwarzkopf (1975) and Schwarzkopf and Fels (1991), with calculation over spectral bands associated with carbon dioxide, water vapor, and ozone. Included also are Schwarzkopf and Fels (1985) transmission coefficients for carbon dioxide, a Roberts et al. (1976) treatment of the water vapor continuum, as well as the overlap effects of water vapor and carbon dioxide, and of a Voigt line-shape correction.

- Interaction of radiation with clouds follows the delta-Eddington approach (cf. Joseph et al. 1976). Cloud shortwave optical depth is specified for convective cloud and for warm low, middle, and high stratiform clouds and precipitating high clouds, including anvil cirrus, but shortwave optical depth depends on temperature for other subfreezing clouds following Harshvardhan et al. (1989). Both shortwave and longwave cloud optical properties (e.g., shortwave reflectivities and absorptivities and longwave emissivities) are linked to the cloud shortwave optical depth and to liquid/ ice water path following parameterizations of Stephens (1978b) and Ramaswamy and Ramanathan (1989). For purposes of the radiation calculations, all clouds are assumed to be randomly overlapped in the vertical. See also Cloud Formation.

\section{Convection}

A convective scheme after Manabe et al. (1965) performs moist static adjustment of saturated, unstable layers and of supersaturated stable layers. With use of the 
Mellor and Yamada (1982) turbulence closure scheme (see Diffusion), dry convective adjustment is not explicitly performed (however, a radiative cooling adjustment for clouds at least 2 layers thick does not permit the lapse rate to exceed dry adiabatic). Simulation of shallow convection is parameterized in terms of the vertical diffusion, using a method similar to that of Tiedtke (1983).

\section{Cloud Formation}

- Stratiform and convective clouds form according to a modified form of the empirical diagnostic method of Slingo (1987). Some departures from the Slingo scheme include a reduction (from 80 to 70 percent) of the relative-humidity threshold for the formation of stratiform layer cloud and the linear (rather than quadratic) dependence of this cloud amount on the relative humidity above the threshold value (cf. Gordon 1992 for details).

- Clouds are of four types: shallow convective cloud; deep convective cloud; stratiform cloud associated with tropical and extratropical disturbances that forms in low, middle, or high vertical layers; and boundary-layer stratus cloud that is associated with strong temperature inversions. The boundaries for low, middle, and high clouds vary with latitude and season according to climatology (cf. Gordon and Hovanec 1985).

- Convective cloud amount depends on the convective precipitation rate. Nonprecipitating shallow convective cloud amount is determined from a scaled form of the relative-humidity criterion for low layer cloud (see below), and is confined to layers below $750 \mathrm{hPa}$ in regions where a conditionally unstable lapse rate and descent, or weak vertical ascent, are present.

- Low, middle, and high layer cloud is present only when the relative humidity is $>70$ percent, the amount being a linear function of this humidity excess. Low layer cloud forms below $750 \mathrm{hPa}$ only in regions of upward vertical motion. The amount of low and middle layer cloud is reduced in dry downdrafts around subgrid-scale convective clouds. Boundary-layer stratus cloud associated with strong temperature inversions may also form below $750 \mathrm{hPa}$ if the relative humidity is $>60$ percent, the amount depending on this humidity excess and the inversion strength. 


\section{DERF}

\section{Precipitation}

Precipitation from large-scale condensation and from the moist convective adjustment process (see Convection) forms under supersaturated conditions. Subsequent evaporation of falling precipitation is not simulated.

\section{Planetary Boundary Layer}

Conditions within the PBL are typically represented by the first 5 sigma levels above the surface (at sigma $=0.998,0.980,0.948,0.901$, and 0.844). See also Diffusion and Surface Fluxes.

\section{Orography}

Orography obtained from a 1 x 1-degree Scripps dataset (Gates and Nelson 1975) is interpolated to the model's Gaussian grid (see Horizontal Resolution). The heights are then transformed to spectral space and are truncated at $\mathrm{T} 42$ resolution.

\section{Ocean}

AMIP monthly sea surface temperature fields are prescribed, with daily values determined by linear interpolation.

\section{Sea Ice}

AMIP monthly sea ice extents are prescribed. Snow may accumulate on sea ice, but does not alter its thermodynamic properties. The surface temperature of sea ice is prognostically determined after Deardorff (1978) from a surface energy balance (see Surface Fluxes) that includes a conduction heat flux from the ocean below. The conduction flux is proportional to the difference between the surface temperature of the ice and the subsurface ocean temperature (assumed to be at the melting temperature of sea ice, or $271.2 \mathrm{~K}$ ), and the flux is inversely proportional to the constant ice thickness $(2 \mathrm{~m})$. The heat conductivity is assumed to be a constant equal to the value for pure ice, and there is no heat storage within the ice. See also Snow Cover and Surface Characteristics.

\section{Snow Cover}

Precipitation falls as snow if a linear combination of the air temperature on the lowest atmospheric level at sigma $=0.998$ (weighted 0.35) and the temperature on the 
next higher level at sigma $=0.980$ (weighted 0.65 ) is $<0^{\circ} \mathrm{C}$. Snow accumulates on both land and sea ice, and snow mass is determined prognostically from a budget equation that accounts for accumulation and melting. Snow cover affects the surface albedo and the heat transfer/capacity of soil. Sublimation of snow is calculated as part of the surface evaporative flux, and snowmelt contributes to soil moisture. See also Surface Characteristics, Surface Fluxes, and Land Surface Processes.

\section{Surface Characteristics}

- Roughness lengths over oceans are determined from the surface wind stress after the method of Charnock (1955). Roughness lengths are prescribed uniform constants for sea ice $\left(1 \times 10^{-4} \mathrm{~m}\right)$ and land $(0.1682 \mathrm{~m})$.

- Over oceans the surface albedo depends on solar zenith angle (cf. Payne 1972), while the albedo of snow-free sea ice is a constant 0.50 . Albedos for snow-free land are obtained from the data of Posey and Clapp (1964), and do not depend on solar zenith angle or spectral interval.

- Snow cover modifies the local surface background albedo as follows. Poleward of 70 degrees latitude, permanent snow with albedo 0.75 is assumed. Equatorward of 70 degrees, the snow albedo is set to 0.60 if the water-equivalent snow depth is at least a critical value of $0.01 \mathrm{~m}$; otherwise, the albedo is a linear combination of the background and snow albedos weighted by the ratio of snow depth to this critical value.

- Longwave emissivity is prescribed to be unity (blackbody emission) for all surfaces.

\section{Surface Fluxes}

- Surface solar absorption is determined from the surface albedos, and longwave emission from the Planck equation, assuming blackbody emissivity (see Surface Characteristics).

- Surface turbulent eddy fluxes follow Monin-Obukhov similarity theory, as formulated by Delsol et al. (1971). The momentum flux is proportional to the product of a drag coefficient, the wind speed, and the wind velocity vector at the lowest atmospheric level. The surface sensible heat flux is proportional to the product of a transfer coefficient, the wind speed at the lowest atmospheric level, and the vertical difference between the temperature at the surface and that of the lowest level. The drag and transfer coefficients are 
functions of stability (bulk Richardson number) and surface roughness length (see Surface Characteristics).

- The surface moisture flux is the product of potential evaporation and the evapotranspiration efficiency $\beta$. Potential evaporation is proportional to the product of the same transfer coefficient as for the sensible heat flux, the wind speed at the lowest atmospheric level, and the difference between the specific humidity at the lowest level and the saturated specific humidity for the local surface temperature and pressure. The evapotranspiration efficiency $\beta$ is prescribed to be unity over oceans, snow, and ice surfaces. Over land, $\beta$ is a function of the ratio of soil moisture to the constant field capacity (see Land Surface Processes).

- Above the constant-flux surface layer, stability-dependent vertical diffusion of momentum, heat, and moisture follows the Mellor and Yamada (1982) level-2.5 turbulence closure scheme (see Diffusion).

\section{Land Surface Processes}

- Soil temperature is computed after the force-restore method of Deardorff (1978) in three layers with thicknesses of $0.05,0.45$, and 4.5 meters. Soil heat capacity/ conductivity is affected by snow cover through its influence on soil moisture availability in the force-restore formulation (i.e., evapotranspiration efficiency $\beta=1$ for snowcovered surfaces--see Surface Fluxes).

- Soil moisture is represented by the single-layer "bucket" model of Manabe (1969), with field capacity everywhere $0.15 \mathrm{~m}$. Soil moisture is increased by precipitation and snowmelt, and is decreased by surface evaporation, which is determined from a product of the evapotranspiration efficiency $\beta$ and the potential evaporation from a surface saturated at the local surface temperature and pressure (see Surface Fluxes). Over land, $\beta$ is a function of the ratio of local soil moisture to the constant field capacity $(0.15 \mathrm{~m})$, with runoff occurring implicitly if this ratio exceeds unity. 


\title{
DNM: Department of Numerical Mathematics (of the Russian Academy of Sciences)
}

\begin{abstract}
AMIP Representative(s)
Dr. V. Galin and Dr.V. Dymnikov, Institute of Numerical Mathematics, Russian Academy of Sciences, Leninsky Pr., 32a, Moscow 117334, Russia; Phone: +7-095-9381808; Fax: +7-095-938-1821; e-mail: galin@adonis.iasnet.com.
\end{abstract}

Model Designation

DNM A5407.V1 4x5L7

\section{Model Lineage}

The DNM model was initially developed in the early 1980 s by G. I. Marchuk and collaborators (cf. Marchuk 1984).

\section{Model Documentation}

Key documentation of the DNM mode is provided by Marchuk et al. (1984). The radiation scheme is described by Feigelson (1984), Podolskaya and Rivin (1988), and Galin (1984). The treatment of turbulent fluxes in the planetary boundary layer (PBL) follows Lykossov (1990) and Kazakov and Lykossov (1982) .

\section{Numerical/Computational Properties}

\section{Horizontal Representation}

Second-order finite differences on a shifted C-grid (cf. Arakawa and Lamb 1977) with conservation of total atmospheric mass, energy, and potential enstrophy.

\section{Horizontal Resolution \\ $4 \times 5$-degree latitude-longitude grid.}

\section{Vertical Domain}

Surface to about $71 \mathrm{hPa}$. For a surface pressure of $1000 \mathrm{hPa}$, the lowest atmospheric level is at about $929 \mathrm{hPa}$. 


\section{DNM}

\section{Vertical Representation}

Finite differences in sigma coordinates.

\section{Vertical Resolution}

There are 7 regularly spaced sigma levels. For a surface pressure of $1000 \mathrm{hPa}$, one level is below $800 \mathrm{hPa}$ and one level is above $200 \mathrm{hPa}$.

\section{Computer/Operating System}

The AMIP simulation was run on a Cray 2 computer using a single processor in a UNICOS environment.

\section{Computational Performance}

For the AMIP experiment, about 3 minutes Cray 2 computation time per simulated day.

\section{Initialization}

For the AMIP simulation, initial conditions of the model atmosphere, soil moisture, and snow cover/depth for 1 January 1979 are determined from a simulation of October through December 1978, starting from arbitrary initial conditions, but with the ocean surface temperatures and sea ice extents prescribed to be the same as the AMIP boundary conditions for January 1979.

\section{Time Integration Scheme(s)}

Time integration is by the Matsuno scheme, with time steps of 6 minutes for dynamics, 3 hours for radiation, and 1 hour for all other model physics.

\section{Smoothing / Filling}

Orography is smoothed (see Orography). Atmospheric temperature, specific humidity, and u-v winds are filtered at latitudes poleward of 50 degrees. Negative values of atmospheric specific humidity are filled, with conservation of the mass of water vapor in the vertical column.

\section{Sampling Frequency}

For the AMIP simulation, the model history is written every 6 hours. 


\section{Dynamical/Physical Properties}

\section{Atmospheric Dynamics}

Primitive-equation dynamics are expressed in terms of $u-v$ winds, temperature, specific humidity, surface pressure, and vertical motion.

\section{Diffusion}

- Second-order nonlinear horizontal diffusion after Marchuk et al. (1984) is applied to the winds, temperature, and specific humidity on sigma surfaces.

- Vertical diffusion includes use of a turbulent kinetic energy equation and nongradient transport of momentum, heat, and moisture after the method of Lykossov (1990) in the PBL (see Planetary Boundary Layer). Vertical diffusion is not applied above the PBL.

\section{Gravity-wave Drag}

Gravity-wave drag is not modeled.

\section{Solar Constant / Cycles}

The solar constant is the AMIP-prescribed value of $1365 \mathrm{~W} \mathrm{~m}^{-2}$. Both seasonal and diurnal cycles in solar forcing are simulated.

\section{Chemistry}

The carbon dioxide concentration is the AMIP-prescribed value of $345 \mathrm{ppm}$. Zonally averaged total column ozone concentration is specified from the data of Koprova and Uranova (1978), with the zonal vertical profile computed as in Lacis and Hansen (1974). Radiative effects of water vapor are also included, but not the effects of aerosols (see Radiation).

\section{Radiation}

- Shortwave radiation is computed after Manabe and Strickler (1964) and Lacis and Hansen (1974) in two spectral bands--an ultraviolet/visible (UVV) band from 0.2 to 0.9 micron, and a near infrared (NIR) band from 0.9 to 4.0 microns. In the UVV, shortwave radiation is absorbed by ozone, but not by water vapor. Rayleigh scattering and multiple cloud reflection effects also are taken into account 
following Lacis and Hansen (1974). In the NIR, the radiation is absorbed by water vapor and clouds, but the radiative effects of aerosols are not included; the directbeam flux and the flux reflected by clouds and by the Earth's surface are distinguished. NIR scattering by water vapor and cloud droplets is not treated.

- Longwave radiation is computed by the method of Feigelson (1984). The integral transmission functions (including pressure-broadening effects) of carbon dioxide and water vapor are after Podolskaya and Rivin (1988), but the radiative effects of the water vapor continuum are not taken into account. The longwave transmission function of ozone is calculated by the method of Raschke (1973). The longwave optical properties of clouds are obtained from Rodgers (1967). Longwave emissivity of high cloud is prescribed as 0.5 , but middle and low clouds are treated as blackbodies (emissivity of 1.0). For purposes of the radiation calculations, clouds are assumed to be randomly overlapped in the vertical. See also Cloud Formation.

\section{Convection}

- Penetrative convection is simulated after the method of Kuo (1974), and is assumed to occur only in the presence of conditionally unstable layers in the vertical and large-scale net moisture convergence in the horizontal. The associated convective cloud formed at the lifting condensation level is assumed to dissolve instantaneously through lateral mixing, thereby imparting heat and moisture to the environment. In a vertical column the total moisture available from convergence is divided between a fraction $b$ that moistens the environment and the remainder $(1-b)$ that contributes to the latent heating (rainfall) rate. The parameter $b$ is determined after Anthes (1977) as a cubic function of the ratio of the mean relative humidity of the cloud layer to a prescribed critical relative humidity threshold value; if the cloud relative humidity is less than the threshold, $b$ is set to 1 (no heating of the environment).

- Shallow convection is simulated inasmuch as a moist convective adjustment is carried out in the PBL only (see Planetary Boundary Layer).

\section{Cloud Formation}

A subgrid-scale convective cloud fraction is not explicitly calculated. The gridscale cloud fraction is based on the relative humidity diagnostic of Smagorinsky (1960) with a threshold humidity of 100 percent required for condensation. These clouds form at 
three levels (low, middle and high cloud), and are taken to be half the thickness of a sigma layer. Eight fractional cases are distinguished in each grid box: a clear-sky fraction; fractions covered only by high, middle, and low cloud, respectively; fractions covered by high and middle cloud, by high and low cloud, and by middle and low cloud; and a fraction that is covered by cloud at all levels (cf. Galin 1984 for further details). See also Radiation for cloud-radiative interactions.

\section{Precipitation}

Grid-scale precipitation is equal to the amount of condensation necessary to return a supersaturated layer to 100 percent relative humidity. There is no subsequent evaporation of falling precipitation. Convective precipitation is determined from the specification of the Kuo moistening parameter b (see Convection).

\section{Planetary Boundary Layer}

- The top of the PBL is assumed to be at the height of the lowest atmospheric vertical level (at sigma $=0.929$ ). The PBL is treated physically as two sublayers: a lower constant-flux sublayer, and an upper mixed transitional sublayer. In the lower sublayer, with top at height $\mathrm{h}=70 \mathrm{~m}$, the turbulent momentum, heat, and moisture fluxes are calculated following Monin-Obukhov similarity theory (see Surface Fluxes). The wind at height $h$ is taken to be the same as that at the PBL top. The temperature at height $h$ is determined from a continuity equation for the equivalent potential temperature flux. The specific humidity at height $h$ is given by the product of saturated specific humidity at this temperature, and of the relative humidity, which is computed from the arithmetic mean of the inverse values at the PBL top and at the surface.

- In the upper sublayer, the wind speed is constant, but the turning of the wind vector with height depends on latitude and surface type. In this upper sublayer there is also nongradient diffusion of heat, moisture, and momentum. See also Diffusion, Surface Fluxes, and Land Surface Processes.

\section{Orography}

The $1 \times$ 1-degree topographic height data of Gates and Nelson (1975) are smoothed by averaging over each $4 \times 5$-degree grid box. Then a 9-point filter is applied to further smooth data from surrounding grid cells. Finally, a Fourier filter is applied poleward of 50 degrees latitude to eliminate high-frequency orographic variance. 


\section{DNM}

\section{Ocean}

AMIP monthly sea surface temperatures are prescribed, with daily values determined by linear interpolation.

\section{Sea Ice}

Monthly AMIP sea ice extents are prescribed, and the ice depth is taken to be a uniform $2 \mathrm{~m}$. Snow is allowed to accumulate on sea ice (see Snow Cover). The top surface temperature of the ice is predicted from the balance of surface energy fluxes (see Surface Fluxes) including conduction heating from below. The conduction is a function of the temperature gradient across the ice, with the bottom surface temperature fixed at $-2{ }^{\circ} \mathrm{C}$ for other surface types.

\section{Snow Cover}

Precipitation falls as snow if the surface air temperature is $<0^{\circ} \mathrm{C}$. The snow depth is determined prognostically on land, continental ice, and sea ice from the moisture budget equation. Snow, which covers the whole of a grid box (i.e., no fractional coverage), affects the surface albedo (see Surface Characteristics). New snow also decreases the heat conductivity of bare soil up to 33 percent, but with no further decrease occurring as snow accumulates (see Land Surface Processes). Sublimation of snow is calculated as part of the surface evaporative flux (see Surface Fluxes). Melting of snow, which contributes to soil moisture, occurs whenever the surface air temperature is $>0{ }^{\circ} \mathrm{C}$.

\section{Surface Characteristics}

- The surface roughness length is assumed to be different for momentum than for the heat and moisture fluxes (see Surface Fluxes). The momentum roughness length over the ocean is a function of the surface stress given by the Charnock (1955) relation, with coefficient 0.14 . Over ice surfaces, the roughness is prescribed to be a uniform $0.01 \mathrm{~m}$. Over land, the roughness length is a spatially variable function of orography (see Orography). The roughness lengths for surface heat and moisture fluxes are the same, and are equated to the momentum roughness length weighted by a function of the Reynolds number. Observed data reviewed by Garratt (1977) are used to specify this function (cf. Kazakov and Lykossov 1982 for details).

- Surface albedos are prescribed as 0.1 over oceans, 0.6 over ice, 0.2 over bare 
soil. For snow-covered areas (see Snow Cover), the albedo is a linear function of waterequivalent snow depth, with values ranging between 0.2 and 0.6 . These albedos are not a function of solar zenith angle or spectral interval.

- Longwave emissivity is prescribed as unity (i.e., blackbody emission) for all surfaces.

\section{Surface Fluxes}

- Surface solar absorption is determined from specified constant albedos, and longwave emission from the Planck equation with prescribed constant emissivity of 1.0 (see Surface Characteristics).

- Surface turbulent eddy fluxes of momentum, heat, and moisture are simulated by a bulk aerodynamic approximation, with drag and transfer coefficients calculated following Monin-Obukhov similarity theory. Empirical functions of Businger et al. (1971) are matched with a $-1 / 3$ power-law dependence for strong instability (cf. Kazakov and Lykossov 1982); these are used as universal functions of roughness length and of thermal stability to define the vertical profiles of wind, temperature, and humidity in the constant-flux surface layer (see Planetary Boundary Layer). When the wind speed at the top of the surface layer (at height $\mathrm{h}=70 \mathrm{~m}$ ) $>15 \mathrm{~m} \mathrm{~s}^{-1}$, the ocean surface heat and moisture fluxes are augmented by the evaporation of sea spray, which is modeled after Borisenkov and Kuznetsov (1978), based on observations of Monahan (1968). Cf. Kazakov and Lykossov (1980) for further details.

- The surface moisture flux depends on the near-surface wind speed and on the specific humidity difference between the top and bottom of the surface layer, which is a function of surface relative humidity (see Planetary Boundary Layer). Over oceans, sea ice, and snow the surface relative humidity is prescribed as 100 percent, while over land it is a function of the soil moisture (see Land Surface Processes).

\section{Land Surface Processes}

- Soil temperature is computed from a surface energy balance with heat storage in a 1-meter layer, and with deep soil temperature at 1 meter prescribed from data of Legates (1987).

- Soil moisture is predicted by a single-layer "bucket" model with uniform $0.15 \mathrm{~m}$ field capacity after the method of Budyko (1956), but with deep soil moisture also 


\section{DNM}

prescribed from monthly estimates of Mintz and Serafini (1981). The surface relative humidity is a function of the ratio of soil moisture to field capacity (see Surface Fluxes). Runoff occurs implicitly if this ratio exceeds unity. 


\section{ECMWF: European Centre for Medium-Range Weather Forecasts}

\section{AMIP Representative(s)}

Dr. Laura Ferranti and Dr. David Burridge, European Centre for MediumRange Weather Forecasts, Shinfield Park, Reading RG29AX, England; Phone: +44-734-499000; Fax: +44-734-869450; e-mail: Laura.Ferranti@ecmwf.co.uk.

\section{Model Designation}

ECMWF ECMWFcycle 36 T42L19

\section{Model Lineage}

Cycle 36, one of a historical line of ECMWF model versions, first became operational in June 1990.

\section{Model Documentation}

Key documents for the model are ECMWF Research Department $(1988,1991)$ and a series of Research Department memoranda from 1988 to 1990 that are summarized in ECMWF Technical Attachment (1993).

\section{Numerical/Computational Properties}

\section{Horizontal Representation}

Spectral (spherical harmonic basis functions) with transformation to a Gaussian grid for calculation of nonlinear quantities and some physics.

\section{Horizontal Resolution}

Spectral triangular 42 (T42), roughly equivalent to $2.8 \times 2.8$ degrees latitudelongitude.

\section{Vertical Domain}

Surface to $10 \mathrm{hPa}$. For a surface pressure of $1000 \mathrm{hPa}$, the lowest atmospheric level is at about $996 \mathrm{hPa}$. 


\section{ECMWF}

\section{Vertical Representation}

Finite differences in hybrid sigma-pressure coordinates after Simmons and Burridge (1981) and Simmons and Struefing (1981).

\section{Vertical Resolution}

There are 19 irregularly spaced hybrid levels. For a surface pressure of $1000 \mathrm{hPa}$, 5 levels are below $800 \mathrm{hPa}$ and 7 levels are above $200 \mathrm{hPa}$.

\section{Computer/Operating System}

The AMIP simulation was run on a Cray 2 computer using a single processor in the UNICOS environment.

\section{Computational Performance}

For the AMIP experiment, about 15 minutes of Cray 2 computation time per simulated day.

\section{Initialization}

For the AMIP simulation start date of 1 January 1979, the model atmosphere, soil moisture, snow cover/depth are initialized from ECMWF operational analyses for 15 January 1979 that are interpolated from spectral T106 resolution to T42 (see Horizontal Resolution).

Time Integration Scheme(s)

A semi-implicit Hoskins and Simmons (1975) scheme with Asselin (1972) frequency filter is used for the time integration, with a time step of 30 minutes for dynamics and physics, except for radiation/cloud calculations, which are done once every 3 hours.

\section{Smoothing / Filling}

Orography is smoothed (see Orography). Negative values of atmospheric specific humidity (due to truncation errors in the discretized moisture equation) are filled by borrowing moisture from successive vertical levels below until all specific humidity values in the column are nonnegative. Any borrowing from the surface that may be required does not impact the moisture budget there. 
Sampling Frequency

For the AMIP simulation, the model history is written every 6 hours.

\section{Dynamical/Physical Properties}

\section{Atmospheric Dynamics}

Primitive-equation dynamics are expressed in terms of vorticity, divergence, temperature, surface pressure, and specific humidity.

\section{Diffusion}

- Fourth-order $\left(\nabla^{4}\right)$ horizontal diffusion is applied in spectral space on hybrid vertical surfaces to vorticity, divergence, moisture, and on pressure surfaces to temperature.

- Second-order vertical diffusion operates above the planetary boundary layer (PBL) only in conditions of static instability. In the PBL, vertical diffusion of momentum, heat, and moisture is proportional to the vertical gradients of the wind, specific humidity, and dry static energy, respectively (see Planetary Boundary Layer). The vertically variable diffusion coefficient depends on stability (bulk Richardson number) as well as the vertical shear of the wind, following standard mixing-length theory.

\section{Gravity-wave Drag}

Drag associated with orographic gravity waves is simulated after the method of Palmer et al. (1986), as modified by Miller et al. (1989), using directionally dependent subgrid-scale orographic variances obtained from the U.S. Navy dataset (cf. Joseph 1980). Surface stress due to gravity waves excited by stably stratified flow over irregular terrain is calculated from linear theory and dimensional considerations. Gravity-wave stress is a function of atmospheric density, low-level wind, and the Brunt-Vaisalla frequency. The vertical structure of the momentum flux induced by gravity waves is calculated from a local wave Richardson number, which describes the onset of turbulence due to convective instability and the turbulent breakdown approaching a critical level. 


\section{ECMWF}

\section{Solar Constant/Cycles}

The solar constant is the AMIP-prescribed value of $1365 \mathrm{~W} \mathrm{~m}^{-2}$. Both seasonal and diurnal cycles in solar forcing are simulated.

\section{Chemistry}

The carbon dioxide concentration is the AMIP-prescribed value of $345 \mathrm{ppm}$. The ozone profile is determined from total ozone in a column (after data by London et al. 1976) and the height of maximum concentration (after data by Wilcox and Belmont 1977), and depends on pressure, latitude, longitude, and season. Mie radiative parameters of five types of aerosol (concentration depending only on height) are provided from WMOICSU (1984) data. Radiative effects of water vapor, carbon monoxide, methane, nitrous oxide, and oxygen are also included (see Radiation).

\section{Radiation}

- Atmospheric radiation is simulated after the method of Morcrette $(1989,1990$, 1991). For clear-sky conditions, shortwave radiation is modeled by a two-stream formulation in spectral wavelength intervals $0.25-0.68$ micron and 0.68-4.0 microns using a photon path distribution method to separate the effects of scattering and absorption processes. Shortwave absorption by water vapor, ozone, oxygen, carbon monoxide, methane, and nitrous oxide is included using line parameters of Rothman et al. (1983). Rayleigh scattering and Mie scattering/absorption by five aerosol types are treated by a delta-Eddington approximation.

- The clear-sky longwave scheme employs a broad-band flux emissivity method in six spectral intervals from wavenumbers 0 to $2.6 \times 10^{5} \mathrm{~m}^{-1}$, with continuum absorption by water vapor included from wavenumbers $3.5 \times 10^{4}$ to $1.25 \times 10^{5} \mathrm{~m}^{-1}$. The temperature/pressure dependence of longwave gaseous absorption follows Morcrette et al. (1986). Aerosol absorption is also modeled by an emissivity formulation.

- Shortwave scattering and absorption by cloud droplets is treated by a deltaEddington approximation; radiative parameters include optical thickness, singlescattering albedo linked to cloud liquid water path, and prescribed asymmetry factor. Cloud types are distinguished by defining shortwave optical thickness as a function of effective droplet radius. Clouds are treated as graybodies in the longwave, with 
emissivity depending on cloud liquid water path after Stephens (1978b). Longwave scattering by cloud droplets is neglected, and droplet absorption is modeled by an emissivity formulation from the cloud liquid water path. For purposes of the radiation calculations, clouds of different types are assumed to be randomly overlapped in the vertical, while convective cloud and nonconvective cloud of the same type in adjacent layers are treated as fully overlapped. See also Cloud Formation.

\section{Convection}

The mass-flux convective scheme of Tiedtke (1989) accounts for midlevel and penetrative convection, and also includes effects of cumulus-scale downdrafts. Shallow (stratocumulus) convection is parameterized by means of an extension of the model's vertical diffusion scheme (cf. Tiedtke et al. 1988). The closure assumption for midlevel/penetrative convection is that large-scale moisture convergence determines the bulk cloud mass flux; for shallow convection, the mass flux is instead maintained by moisture from surface evaporation. Entrainment/detrainment of mass in convective plumes occurs both through turbulent exchange and organized inflow/outflow. Momentum transport by convective circulations is also included, following Schneider and Lindzen (1976).

\section{Cloud Formation}

- Cloud formation follows the diagnostic method of Slingo (1987). Clouds are of three types: shallow, midlevel, and high convective cloud; cloud associated with fronts/tropical disturbances that forms in low, medium, or high vertical layers; and low cloud associated with temperature inversions.

- The height of midlevel/high convective cloud is determined by the level of nonbuoyancy for moist adiabatic ascent (see Convection), and the cloud amount (fractional area $0.2-0.8$ ) from the scaled logarithm of the convective precipitation rate. If this convective cloud forms above $400 \mathrm{hPa}$ and the fractional area is $>0.4$, anvil cirrus cloud also forms. Shallow convective cloud amount is determined from the difference between the moisture flux at cloud base and cloud top.

- Frontal cloud is present only when the relative humidity is $>80$ percent, the amount being a quadratic function of this humidity excess. Low frontal cloud is absent in regions of grid-scale subsidence, and the amount of low and middle frontal cloud is reduced in dry downdrafts around subgrid-scale convective clouds. In a 
temperature inversion, low cloud forms if the relative humidity is $>60$ percent, the amount depending on this humidity excess and the inversion strength. See also Radiation for treatment of cloud-radiative interactions.

\section{Precipitation}

- Freezing/melting processes in convective clouds are not considered. Conversion from cloud droplets to raindrops is proportional to the cloud liquid water content. No liquid water is stored in a convective cloud, and once detrained, it evaporates instantaneously with any portion not moistening the environment falling out as subgridscale convective precipitation. Evaporation of convective precipitation is parameterized (following Kessler 1969) as a function of convective rain intensity and saturation deficit (difference between saturated specific humidity and that of environment).

- Precipitation also results from gridscale condensation when the local specific humidity exceeds the saturated humidity at ambient temperature/pressure; the amount of precipitation depends on the new equilibrium specific humidity resulting from the accompanying latent heat release. Before falling to the surface, gridscale precipitation must saturate all layers below the condensation level by evaporation. See also Convection and Cloud Formation.

\section{Planetary Boundary Layer}

The PBL is represented typically by the first 5 vertical levels above the surface (at about 996, 983, 955, 909, and $846 \mathrm{hPa}$ for a surface pressure of $1000 \mathrm{hPa}$, or at approximate elevations of $30 \mathrm{~m}, 150 \mathrm{~m}, 400 \mathrm{~m}, 850 \mathrm{~m}$, and $1450 \mathrm{~m}$, respectively). The PBL height is diagnostically determined as the greater of the height predicted from Ekman theory versus a convective height that depends on dry static energy in the vertical.

\section{Orography}

Orography is obtained from a U.S. Navy dataset (cf. Joseph 1980) with resolution of 10 minutes arc on a latitude/longitude grid. The mean terrain heights are then calculated for a T106 Gaussian grid, and the square root of the corresponding subgrid-scale orographic variance is added. The resulting "envelope orography" (cf. Wallace et al. 1983) is smoothed by application of a Gaussian filter with a $50 \mathrm{~km}$ radius of influence (cf. Brankovic and Van Maanen 1985). This filtered orography is 
then spectrally fitted and truncated at the T42 resolution of the model. See also Gravity-wave Drag.

\section{Ocean}

AMIP monthly sea surface temperature fields are prescribed, with daily values determined by linear interpolation.

\section{Sea Ice}

AMIP monthly sea ice extents are prescribed. The surface temperature of the ice is specified from monthly climatologies. Snow is not allowed to accumulate on sea ice (see Snow Cover).

\section{Snow Cover}

Grid-scale precipitation may fall as snow if the temperature of the layer of its formation is $<0^{\circ} \mathrm{C}$. Convective precipitation changes to snow only if the surface air temperature is $<-3{ }^{\circ} \mathrm{C}$, and over land only if the ground temperature is $<0{ }^{\circ} \mathrm{C}$. Snow depth (measured in meters of equivalent liquid water) is determined prognostically from a budget equation, with accumulation allowed only on land surfaces. The fractional area of snow coverage of a grid square is given by the ratio of the snow depth to a critical water-equivalent depth $(0.015 \mathrm{~m})$, or is set to unity if the snow depth exceeds this critical value. Sublimation of snow is calculated as part of the surface evaporative flux (see Surface Fluxes). Snow cover also alters the surface albedo (see Surface Characteristics) and the heat conductivity of the soil (see Land Surface Processes). Melting of snow (which contributes to soil moisture) occurs whenever the ground temperature exceeds $+2{ }^{\circ} \mathrm{C}$.

\section{Surface Characteristics}

- The fractional area of vegetation (undistinguished by type) on each grid square is determined from Matthews (1983) 1 x 1-degree data, as modified by Wilson and Henderson-Sellers (1985).

- The roughness length is prescribed as $1 \times 10^{-3} \mathrm{~m}$ over sea ice. It is computed over open ocean from the variable surface wind stress by the method of Charnock (1955), but is constrained to be at least $1.5 \times 10^{-5} \mathrm{~m}$. Over land, the roughness length is 


\section{ECMWF}

prescribed as a blended function of local orographic variance, vegetation, and urbanization (cf. Tibaldi and Geleyn 1981, Baumgartner et al. 1977, and Brankovic and Van Maanen 1985) that is interpolated to the model grid. The logarithm of local roughness length then is smoothed by the same Gaussian filter used for the orography (see Orography).

- Annual means of satellite-observed surface albedo (range 0.07 to 0.80 ) from data of Preuss and Geleyn (1980) and Geleyn and Preuss (1983) are interpolated to the model grid and smoothed by the same Gaussian filter as for orography (see Orography). Snow cover alters this background albedo: snow albedo (maximum 0.80) varies depending on depth, masking by vegetation, temperature, and the presence of ice dew (see Snow Cover). Sea ice albedo is prescribed as 0.55 , and ocean albedo as 0.07 . Albedos do not depend on solar zenith angle or spectral interval.

- Longwave emissivity is prescribed as 0.996 on all surfaces. Cf. ECMWF Research Department (1991) for further details.

\section{Surface Fluxes}

- Surface solar absorption is determined from surface albedo, and longwave emission from the Planck equation with prescribed constant surface emissivity (see Surface Characteristics).

- Surface eddy fluxes of momentum, heat, and moisture are expressed as bulk formulae, following Monin-Obukhov similarity theory. The near-surface wind, temperature, and moisture required for the bulk formulae are taken to be the values at the lowest atmospheric level (at about $996 \mathrm{hPa}$ for a surface pressure of $1000 \mathrm{hPa}$ ). The drag and transfer coefficients are functions of stability (bulk Richardson number) and roughness length (see Surface Characteristics), following the method of Louis (1979) and Louis et al. (1981), but with modifications by Miller et al. (1992) for calm conditions over the oceans. The transfer coefficient for moisture is the same as that for heat.

- The surface specific humidity over the ocean and snow-covered areas is the saturated value for the local surface temperature and pressure; over bare soil it is the product of the local saturated value and the surface relative humidity. The moisture flux over vegetation is given by the vertical difference of the specific humidity at the lowest atmospheric level and the saturated value at the surface temperature and pressure, all multiplied by an evapotranspiration efficiency factor $\beta$ (cf. Budyko 1974). 
This efficiency is the inverse sum of the aerodynamic resistance (surface drag) and the stomatal resistance, which depends on radiation stress, canopy moisture, and soil moisture stress in the vegetation root zone (cf. Sellers et al. 1986, Blondin 1989, and Blondin and Boettger 1987). See also Land Surface Processes.

\section{Land Surface Processes}

- Soil temperature and moisture are predicted in two layers of thicknesses $0.07 \mathrm{~m}$ and $0.42 \mathrm{~m}$ that overlie a deep layer (of thickness $0.42 \mathrm{~m}$ ) in which temperature and moisture are prescribed from monthly climatologies (cf. Blondin and Boettger 1987, Brankovic and Van Maanen 1985, Mintz and Serafini 1981). The upper boundary condition for the soil heat diffusion is the net surface energy balance (see Surface Fluxes). Soil heat capacity and diffusivity are functions of snow cover, and the diffusivity is also a function of vegetation canopy area.

- The vegetation canopy also intercepts a fraction of the total precipitation (which is subject to immediate evaporation) that would otherwise infiltrate the soil. The infiltrated soil moisture obeys a simple diffusion equation modified by gravitational effects (Darcy's Law), and is also affected by evaporation from the bare soil portion of each grid box as well as evapotranspiration by vegetation (see Surface Fluxes). Runoff occurs if the maximum soil moisture capacity of the surface layer $(0.02 \mathrm{~m})$ or middle layer $(0.12 \mathrm{~m})$ is exceeded; the fraction of infiltrated moisture associated with the surface runoff due to sloping terrain is also simulated using orographic variance data (see Orography). 


\section{GFDL: Geophysical Fluid Dynamics Laboratory}

\section{AMIP Representative(s)}

Dr. Richard Wetherald, Geophysical Fluid Dynamics Laboratory/NOAA, Princeton University, P.O. Box 308, Princeton, New Jersey 08540; Phone: +1-609-258-6515; Fax: +1-609-987-5063; e-mail: rw@GFDL.GOV

\section{Model Designation \\ GFDL CDG1 R30L14}

\section{Model Lineage}

One of several versions of global spectral models in use at the Geophysical Fluid Dynamics Laboratory, the GFDL model is applied primarily to climate studies. In its treatment of atmospheric dynamics, the model is similar to the GFDL Dynamical Extended-Range Forecasting (DERF) model (cf. Gordon and Stern 1982 and Miyakoda and Sirutis 1986), but it displays a number of differences in numerical features (e.g., use of rhomboidal rather than triangular spectral truncation and a somewhat coarser vertical resolution), as well as in some model parameterizations (e.g., radiation and vertical diffusion).

\section{Model Documentation}

Key documentation of dynamical features is given by Gordon and Stern (1982) and Manabe and Hahn (1981). Physical parameterizations are described by Manabe (1969), Holloway and Manabe (1971), Manabe and Holloway (1975), Manabe et al. (1965), Broccoli and Manabe (1992), Wetherald and Manabe (1988), and Wetherald et al. (1991).

\section{Numerical/Computational Properties}

\section{Horizontal Representation}

Spectral (spherical harmonic basis functions) with transformation to a Gaussian grid for calculation of nonlinear quantities and some physics. 


\section{Horizontal Resolution}

Spectral rhomboidal 30 (R30), roughly equivalent to $2.25 \times 3.75$ degrees latitudelongitude.

\section{Vertical Domain}

Surface to about $15 \mathrm{hPa}$. For a surface pressure of $1000 \mathrm{hPa}$, the lowest atmospheric level is at about $997 \mathrm{hPa}$.

\section{Vertical Representation}

Finite-difference sigma coordinates.

\section{Vertical Resolution}

There are 14 unevenly spaced sigma levels. For a surface pressure of $1000 \mathrm{hPa}$, 4 levels are below $800 \mathrm{hPa}$ and 4 levels are above $200 \mathrm{hPa}$.

\section{Computer/Operating System}

The AMIP simulation was run on a Cray Y/MP computer using a single processor in a UNICOS operating environment.

\section{Computational Performance}

For the AMIP experiment, about 1 minute of Cray Y/MP computation time per simulated day.

\section{Initialization}

For the AMIP simulation, the model atmosphere, soil moisture, and snow cover/ depth are initialized for 1 January 1979 from a previous 10-year integration of the model with climatological sea surface temperatures (cf. Alexander and Mobley 1976).

\section{Time Integration Scheme(s)}

A leapfrog semi-implicit scheme similar to that of Bourke (1974) with an Asselin (1972) frequency filter is used for time integration. The time step is 18 minutes for dynamics and physics, except for full calculations of all radiative fluxes, which are done once every 24 hours. 


\section{GFDL}

\section{Smoothing / Filling}

Orography is smoothed (see Orography). Negative moisture values (that arise because of spectral truncation) are filled by borrowing from nearest neighbors in the vertical, and horizontally in the east-west direction only.

\section{Sampling Frequency}

For the AMIP simulation, the model history is written once every 24 hours.

\section{Dynamical/Physical Properties}

\section{Atmospheric Dynamics}

Primitive-equation dynamics are expressed in terms of vorticity, divergence, surface pressure, specific humidity, and temperature (with a linearized correction for virtual temperature in diagnostic quantities, where applicable).

\section{Diffusion}

- Linear fourth-order $\left(\nabla^{4}\right)$ horizontal diffusion is applied on constant pressure surfaces to vorticity, divergence, temperature, and specific humidity.

- Second-order vertical diffusion with coefficients derived from mixing-length considerations is applied to momentum, heat, and moisture on the first 7 vertical levels above the surface (up to a height of about $5 \mathrm{~km}$ ). The vertical diffusion is not stability-dependent.

\section{Gravity-wave Drag}

The parameterization of orographic gravity-wave drag follows linear theory, as formulated by Y. Hayashi (cf. Broccoli and Manabe 1992). The drag is given by the vertical divergence of the wave stress, which near the surface is equal to the product of the subgridscale orographic variance and a representative mountain wavenumber with a massweighted average of the atmospheric density, the Brunt-Vaisalla frequency, and the wind in the first three layers above the surface (centered on sigma $=0.9353,0.97865$, and 0.99665 ). The wave stress is assumed to be zero above a critical sigma level where the projection of the wind on the surface wind vector vanishes. Below this, the stress increases linearly to its surface value as the sigma-distance from this critical level increases. 


\section{Solar Constant / Cycles}

The solar constant is the AMIP-prescribed value of $1365 \mathrm{~W} \mathrm{~m}^{-2}$. A seasonal, but not a diurnal cycle in solar forcing, is simulated.

\section{Chemistry}

The carbon dioxide concentration is the AMIP-prescribed value of $345 \mathrm{ppm}$. Zonally averaged seasonal mean ozone distributions are specified as a function of height after data of Hering and Borden (1965). These data are linearly interpolated for intermediate times. Radiative effects of water vapor, but not those of aerosols, also are included (see Radiation).

\section{Radiation}

- Shortwave Rayleigh scattering, and absorption in ultraviolet (wavelengths less than 0.35 micron) and visible (wavelengths between 0.5 to 0.7 micron) spectral bands by ozone, and in the near-infrared (wavelengths 0.7 to 4.0 microns) by water vapor are treated by a method similar to that of Lacis and Hansen (1974). Pressure corrections and multiple reflections between clouds and the surface are treated, but radiative effects of aerosols are not included.

- Longwave radiation follows the method of Rodgers and Walshaw (1966), as modified by Stone and Manabe (1968). Absorption by water vapor, carbon dioxide, and ozone is included. The 6.3-micron band, the rotation band, and the continuum of water vapor are subdivided into 19 subintervals, two of which contain the 15-micron carbon dioxide and 9.6-micron ozone absorption bands, with transmissions of the latter two gases multiplied together in overlapping bands. A random model is used to represent the absorptivity for each subinterval, and the Curtis-Godson approximation is used to estimate the effective pressure for absorption. The temperature dependence of line intensity is also incorporated. Carbon dioxide absorptivity is obtained from data of Burch et al. (1961), with its temperature dependence following the model of Sasamori (1959). The ozone absorptivity is obtained from data of Walshaw (1957).

- Cloud-radiative interactions are treated as described by Wetherald and Manabe (1988) and Wetherald et al. (1991). Cloud optical properties (absorptivity/ reflectivity in the ultraviolet-visible and near-infrared intervals, and emissivity in the longwave) depend on cloud height (high, middle, and low) and thickness. The values 


\section{GFDL}

of shortwave properties are assigned following Rodgers (1967a); in the longwave, the emissivity of thin high clouds (above $10.5 \mathrm{~km}$ ) is prescribed as 0.6 after Kondratiev (1972), while all other clouds are treated as blackbodies (emissivity $=1.0$ ). No partial cloudiness is accounted for in each grid box; clouds, therefore, are treated as fully overlapped in the vertical. See also Cloud Formation.

\section{Convection}

Penetrative convection is simulated via convective adjustment processes. If the lapse rate exceeds dry adiabatic, a convective adjustment restores the lapse rate to dry adiabatic, with conservation of dry static energy in the vertical. A moist convective adjustment scheme after Manabe et al. (1965) also operates when the lapse rate exceeds moist adiabatic and the air is supersaturated. (For supersaturated stable layers, nonconvective large-scale condensation takes place--see Precipitation). In moist convective layers it is assumed that the intensity of convection is strong enough to eliminate the vertical gradient of potential temperature instantaneously, while conserving total moist static energy. It is further assumed that the relative humidity in the layer is maintained at 100 percent, owing to the vertical mixing of moisture, condensation, and evaporation from water droplets. Shallow convection is not explicitly simulated.

\section{Cloud Formation}

Cloud forms when the relative humidity of a vertical layer exceeds a heightdependent threshold, whether this results from large-scale condensation or moist convective adjustment (see Convection). (The threshold relative humidity varies linearly between 100 percent at the bottom of the model atmosphere to 90 percent at its top.) It is assumed that no condensed water is retained as cloud liquid water, but immediately precipitates (see Precipitation). Cloud type is defined according to height: high cloud forms above $10.5 \mathrm{~km}$, middle cloud between 4.0 to $10.5 \mathrm{~km}$, and low cloud between 0.0 to $4.0 \mathrm{~km}$. Cloud may form in a single layer or in multiple contiguous layers; cloud occupying a single layer is treated as radiatively thin, and otherwise as radiatively thick. Clouds are assumed to fill the whole grid box (cloud fraction $=1$ ), and therefore to be fully overlapped in the vertical. Cf. Wetherald and Manabe (1988) and Wetherald et al. (1991) for further details. See also Radiation. 


\section{Precipitation}

Precipitation from large-scale condensation and from the moist convective adjustment process (see Convection) forms under supersaturated conditions. Subsequent evaporation of falling precipitation is not simulated.

\section{Planetary Boundary Layer}

The PBL is simulated only in a rudimentary way (e.g., above the surface layer vertical diffusion of momentum, heat, and moisture are not stability-dependent--see Diffusion). The PBL top is not computed, but its dynamics and physics are typically represented by the first 5 vertical levels above the surface (at about sigma $=0.997$, $0.979,0.935,0.866$, and 0.777). See also Surface Characteristics and Surface Fluxes.

\section{Orography}

Raw orography data obtained from the U.S. Navy dataset (cf. Joseph 1980) are smoothed by application of a Fourier filter in the east-west direction, and then are transformed to spectral space at the (R30) model resolution. Subgrid-scale orographic variances required for the gravity-wave drag parameterization are obtained from the same dataset (see Gravity-wave Drag).

\section{Ocean}

AMIP monthly sea surface temperature fields are prescribed, with daily values determined by linear interpolation.

\section{Sea Ice}

AMIP monthly sea ice extents are prescribed. Snow may accumulate on sea ice, but does not alter its thermodynamic properties. The surface temperature of sea ice is prognostically determined after Holloway and Manabe (1971) from a surface energy balance (see Surface Fluxes) that includes a conduction heat flux from the ocean below. The conduction flux is proportional to the difference between the surface temperature of the ice and the subsurface ocean temperature (assumed to be at the melting temperature of sea ice, or $271.2 \mathrm{~K}$ ), and the flux is inversely proportional to the constant ice thickness $(2 \mathrm{~m})$. The heat conductivity is assumed to be a constant equal to the value for pure ice, and there is no heat storage within the ice. 


\section{GFDL}

\section{Snow Cover}

Precipitation falls as snow if the air temperature at $350 \mathrm{~m}$ above the surface (sigma $=0.9575)$ is $<0^{\circ} \mathrm{C}$. Snow accumulates on both land and sea ice (see Sea Ice), and snow mass is determined prognostically from a budget equation that accounts for accumulation, melting, and sublimation. Sublimation is calculated as part of the surface evaporative flux. Snowmelt, determined from the excess surface energy available for a surface at temperature $273.2 \mathrm{~K}$, contributes to soil moisture (cf. Holloway and Manabe 1971). Snow cover affects the albedo of the surface, but not its thermodynamic properties (see Surface Characteristics, Surface Fluxes, and Land Surface Processes).

\section{Surface Characteristics}

- Roughness lengths over ocean are determined from the surface wind stress after the method of Charnock (1955). Roughness lengths are prescribed constants for sea ice $\left(1 \times 10^{-4} \mathrm{~m}\right)$ and land $(0.1682 \mathrm{~m})$.

- Over oceans, the surface albedo depends on solar zenith angle (cf. Payne 1972). The albedo of snow-free sea ice is a constant 0.50 . Albedos for snow-free land are obtained from the data of Posey and Clapp (1964), and do not depend on solar zenith angle or spectral interval.

- Snow cover modifies the local background albedo of the surface according to its depth, following Holloway and Manabe (1971). Poleward of 75 degrees latitude, permanent snow with albedo 0.80 is assumed. Equatorward of 75 degrees, snow albedo is set to 0.60 if the water-equivalent snow depth is at least a critical value of $0.01 \mathrm{~m}$; otherwise, the albedo is a combination of the background and snow albedos weighted by the square root of the water-equivalent snow depth.

- Longwave emissivity is prescribed as unity (blackbody emission) for all surfaces.

\section{Surface Fluxes}

- Surface solar absorption is determined from the surface albedos, and longwave emission from the Planck equation, assuming blackbody emissivity (see Surface Characteristics).

- Surface turbulent eddy fluxes follow Monin-Obukhov similarity theory, as formulated by Delsol et al. (1971). The momentum flux is proportional to the product 
of a drag coefficient, the wind speed, and the wind velocity vector at the lowest atmospheric level (at about sigma $=0.997$ ). Surface sensible heat flux is proportional to the product of a transfer coefficient, the wind speed at the lowest atmospheric level, and the vertical difference between the temperature at the surface and that of the lowest level. The drag and transfer coefficients are functions of stability (bulk Richardson number) and surface roughness length (see Surface Characteristics).

- The surface moisture flux is the product of potential evaporation and evapotranspiration efficiency $\beta$. Potential evaporation is proportional to the product of the same transfer coefficient as for the sensible heat flux, the wind speed at the lowest atmospheric level, and the difference between the specific humidity at the lowest level and the saturated specific humidity for the local surface temperature and pressure. The evapotranspiration efficiency $\beta$ is prescribed to be unity over oceans, snow, and ice surfaces; over land, $\beta$ is a function of the ratio of soil moisture to the constant field capacity (see Land Surface Processes).

-Above the constant-flux surface layer, the vertical diffusion of momentum, heat, and moisture is not stability-dependent (see Diffusion).

\section{Land Surface Processes}

- Ground temperature is determined from a surface energy balance (see Surface Fluxes) without provision for soil heat storage.

- Soil moisture is represented by the single-layer "bucket" model of Manabe (1969), with field capacity everywhere $0.15 \mathrm{~m}$. Soil moisture is increased by precipitation and snowmelt; it is depleted by surface evaporation, which is determined from a product of the evapotranspiration efficiency $\beta$ and the potential evaporation from a surface saturated at the local surface temperature and pressure (see Surface Fluxes). Over land, $\beta$ is given by the ratio of local soil moisture to a critical value that is 75 percent of field capacity, and is set to unity if soil moisture exceeds this value. Runoff occurs implicitly if soil moisture exceeds the field capacity. 


\title{
GISS: Goddard Institute for Space Studies
}

\author{
AMIP Representative(s) \\ Dr. K. Kenneth Lo, NASA Goddard Institute for Space Studies, 2880 Broadway, \\ New York, New York 10025; Phone: +1-212-678-5609; Fax: +1-212-678-5552; \\ e-mail: cdkk1@nasagiss.giss.nasa.gov
}

\section{Model Designation \\ GISS Model II Prime 4x 5L9}

\section{Model Lineage}

The GISS model used for the AMIP experiment is a modified version of Model II that is described by Hansen et al. (1983). The current model differs from this predecessor principally in numerics and in the treatment of convection, planetary boundary layer (PBL), large-scale clouds, and ground hydrology.

\section{Model Documentation}

Basic documentation of the model is provided by Hansen et al. (1983), with subsequent changes to the convective scheme described by Del Genio and Yao (1988), and Yao and Del Genio (1989). Early versions of the current large-scale cloud parameterization and ground hydrology scheme are described, respectively, by Del Genio et al. (1993) and by Abramopoulos et al. (1988).

\section{Numerical/Computational Properties}

\section{Horizontal Representation}

Atmospheric mass and zonal and meridional velocity components are represented on a B-grid (cf. Arakawa 1972), which conserves mass, kinetic energy (but not angular momentum) under advection, and enstrophy in the nondivergent limit. The prognostic variable for mass (see Dynamics) represents a mean value over a grid box. For heat and moisture prognostics, however, the linear gradients and second-order moments in three dimensions, and the three cross-term second-order moments are included in addition to the mean quantity for each grid box. (Potential enthalpy and water vapor 
are advected via a stable and accurate quadratic upstream scheme that utilizes these first- and second-order moments.)

\section{Horizontal Resolution}

$4 \times 5$-degree latitude-longitude grid.

\section{Vertical Domain}

Surface to $10 \mathrm{hPa}$. For a surface pressure of $1000 \mathrm{hPa}$, the first vertical level above the surface is at about $975 \mathrm{hPa}$.

\section{Vertical Representation}

Finite-difference sigma coordinates up to $10 \mathrm{hPa}$, the model top for dynamics. Above $10 \mathrm{hPa}$ the atmosphere interacts only radiatively with lower levels (i.e., its temperature profile here is determined solely by radiation).

\section{Vertical Resolution}

There are 9 unevenly spaced sigma levels (see Vertical Representation). For a surface pressure of $1000 \mathrm{hPa}, 2$ levels are below $800 \mathrm{hPa}$ and 2 levels are above $200 \mathrm{hPa}$.

Computer/Operating System

The AMIP simulation was run on an IBM PowerStation 580 computer, using RISC/6000 processors in a UNIX environment.

\section{Computational Performance}

For the AMIP experiment, about 10 minutes of IBM Powerstation 580 computer time per simulated day.

\section{Initialization}

For the AMIP experiment, the model atmospheric state, soil moisture, and snow cover/depth are initialized for 1 January 1979 from a previous model simulation of December. 


\section{GISS}

\section{Time Integration Scheme(s)}

Time integration is by a leapfrog scheme that is initiated each hour with an Euler-backward step. The dynamical time step is 7.5 minutes, while the physical source terms (except radiation) are updated hourly. (The hourly update of the PBL source term employs 4 successive 15 -minute time steps.) Full radiation calculations are performed once every 5 hours.

\section{Smoothing / Filling}

The raw orography data is area-averaged (see Orography). An eighth-order Shapiro (1970) filter is used to smooth the surface pressure. Because the quadratic upstream scheme (see Horizontal Representation) does not guarantee positivedefinite atmospheric moisture, the first- and second-order moments of water vapor mixing ratios are minimally reduced, if necessary, at each advective step to maintain non-negative values.

\section{Sampling Frequency}

For the AMIP simulation, daily averages of selected variables are saved as model history.

\section{Dynamical/Physical Properties}

\section{Atmospheric Dynamics}

Primitive-equation dynamics are expressed in terms of wind velocity, potential temperature, water-vapor mixing ratio, geopotential, pressure, and atmospheric mass (or PS-PT, where PS is the surface pressure and PT is a constant $10 \mathrm{hPa}$ at the dynamical top--see Vertical Representation).

\section{Diffusion}

Neither horizontal nor vertical diffusion is explicitly modeled. However, there is horizontal transport of momentum associated with the convective mass flux (see Convection), and the quadratic upstream advective scheme (see Horizontal Representation) is weakly diffusive as well. 
Gravity-wave Drag

A momentum drag proportional to air density and the square of the velocity is introduced in the top layer of the model (cf. Hansen et al. 1983).

\section{Solar Constant/Cycles}

The solar constant is the AMIP-prescribed value of $1365 \mathrm{~W} \mathrm{~m}^{-2}$. Both seasonal and diurnal cycles in solar forcing are simulated.

\section{Chemistry}

The concentration of carbon dioxide is the AMIP-prescribed value of $345 \mathrm{ppm}$. Ozone concentrations are prescribed as a function of season, height, and geographic location, with column abundances obtained from the climatology of London et al. (1976). Radiative effects of water vapor, and of methane, nitrous oxide, nitric oxide, oxygen, and aerosols also are included (see Radiation).

\section{Radiation}

- A correlated $\mathrm{k}$-distribution method that is a generalization of the approach of Lacis and Hansen (1974) is used for both shortwave and longwave radiative calculations. The k-distribution for a given gas and frequency interval is obtained by leastsquares fitting to calculations of line-by-line absorption for a range of temperatures and pressures by McClatchey et al. (1973) and Rothman (1981).

- The shortwave radiative fluxes are calculated after Lacis and Hansen (1974), but with modifications to obtain accurate results at all solar zenith angles and optical thicknesses. Gaseous absorbers include water vapor, carbon dioxide, ozone, oxygen, nitrous oxide, and nitric oxide. Multiple scattering computations are made of $12 \mathrm{k}$-profiles, with strong line (exponential) absorption of the direct solar beam computed separately for water vapor, carbon dioxide, and oxygen. Absorption and scattering by aerosols are also included using radiative properties obtained from Mie calculations for the global aerosol climatology of Toon and Pollack (1976). The spectral dependence of Mie parameters for clouds, aerosols, and Rayleigh scattering is specified in 6 intervals; these are superimposed on the $12 \mathrm{k}$-profiles to account for overlapping absorption.

-In the longwave, the k-distribution method is used to model absorption by 


\section{GISS}

water vapor, carbon dioxide, ozone, nitrous oxide, nitric oxide, and methane (but with scattering effects neglected). A single k-distribution is specified for each gas, with $11 \mathrm{k}$-intervals for water vapor, 10 for carbon dioxide, and 4 for ozone.

- Shortwave optical thickness of large-scale cloud is based on the prognostic cloud water path (see Cloud Formation). The required droplet effective radius is diagnosed from the cloud water content by assuming constant number concentration with different values for land/ocean cloud and liquid/ice cloud (cf. Del Genio et al. 1993). The shortwave optical thickness of a convective cloud is proportional to its pressure depth. Cloud particle phase function and single-scattering albedo are functions of spectral interval, based on Mie computations for cloud droplet data of Squires (1958) and Hansen and Pollack (1970). For purposes of the radiation calculations, partial cloud cover of a grid box is represented as full cloud cover that occurs for a percentage of the time (implemented via a random number generator--cf. Hansen et al. 1983). Longwave effects of clouds are treated by an emissivity formulation, where the longwave cloud properties are self-consistent with the shortwave properties as a result of the application of Mie theory.

\section{Convection}

- Convection is initiated only when the grid box is convectively unstable in the mean. Dry convection can occur below the condensation level if the moist static energy of a parcel exceeds that of the layer above. Moist convection is triggered if the moist static energy of a parcel exceeds the saturation energy value in the layer above and if, in addition, the implied lifting produces saturation; this level defines the cloud base. The convective cloud top is defined as the upper boundary of the highest vertical layer for which the cloud parcel is buoyant.

- The amount of convective mass flux is obtained from a closure assumption that the cloud base is restored to neutral buoyancy relative to the next higher layer. The mass of the rising convective plume is changed by the entrainment of drier environmental air, with associated decreases in buoyancy. The entrainment rate is prescribed for an ensemble of two convective cloud types (entraining and non-entraining). Heating/cooling of the environment occurs through compensating environmental subsidence, detrainment of cloud air at cloud top, a convective-scale downdraft whose mass flux detrains into the cloud base layer, and evaporation of falling condensate (see Precipitation). (Latent heat release serves only to maintain cloud buoyancy.) The convective plume and subsiding 
environmental air transport gridscale horizontal momentum, under the assumption that exchanged air parcels carry with them the momentum of the layer of origin. Cf. Del Genio and Yao (1988) and Yao and Del Genio (1989) for further details.

\section{Cloud Formation}

- Clouds result from either large-scale or convective condensation (see Convection). Condensation of cloud droplets is assumed to occur with respect to the saturated vapor pressure of water if the local temperature is $>-35{ }^{\circ} \mathrm{C}$, and with respect to the mixed-phase pseudo-adiabatic process of Sassen and Dodd (1989) at lower temperatures. Liquid droplets form if the cloud temperature is $>-4{ }^{\circ} \mathrm{C}\left(-10^{\circ} \mathrm{C}\right)$ over ocean (land), and ice forms at temperatures $<-40^{\circ} \mathrm{C}$. At intermediate temperatures either phase may exist, with increasing probability of ice as temperature decreases.

- The local convective cloud fraction is given by the ratio of convective mass flux to the total atmospheric mass of the grid box (see Convection and Atmospheric Dynamics). Large-scale clouds are predicted from a prognostic cloud water budget equation, where fractional cloudiness is an increasing function of relative humidity above a 60-percent threshold. (Upper-tropospheric convective condensate is also detrained into large-scale anvil cloud.) Cloud top entrainment instability is accounted for using a restrictive instability criterion. Cf. Del Genio et al. (1993) for further details. See also Radiation for treatment of cloud-radiative interactions.

\section{Precipitation}

- Precipitation can result either from large-scale or convective condensation (see Cloud Formation and Convection); the amount of condensate is computed from an iterative solution of the Clausius-Clapeyron equation. For large-scale condensation, the prognostic cloud water is converted to rainwater by autoconversion and accretion, and into snow by a seeder-feeder parameterization.

- Evaporation of both the large-scale and convective condensate is modeled, with the residual falling to the surface as rain or snow (see Snow Cover). Evaporation of large-scale precipitation occurs in all unsaturated layers below its origin. The fraction of convective condensate that evaporates below cloud base is equated to the ratio of the convective mass flux to the total air mass of the grid box, while the fraction that evaporates above the cloud base is taken as half this value. 


\section{GISS}

\section{Planetary Boundary Layer}

The PBL top is defined as the height of dry convection (see Convection). The surface wind velocity is determined using parameterizations of a drag law for the surface layer and a spiral layer above. Similarity theory is applied to calculate the turbulent transport coefficients for the spiral layer and to specify instability functions that determine the surface drag coefficient. The surface atmospheric temperature and water-vapor mixing ratio are obtained by equating surface fluxes computed by the bulk aerodynamic method to a diffusive flux from the surface layer into the layer above; the latter flux depends on a stability-dependent vertical diffusion coefficient and the depth of the PBL. See also Surface Fluxes.

\section{Orography}

Orography is specified by area-averaging the 5-minute resolution topographic height data of National Geophysical Data Center (1988) on the 4 x 5-degree model grid (see Horizontal Resolution).

\section{Ocean}

AMIP monthly sea surface temperatures are prescribed, with daily values determined by linear interpolation.

\section{Sea Ice}

AMIP monthly sea ice extents are prescribed. The ice consists of two layers: an upper layer of constant thickness $(0.1 \mathrm{~m})$ and a lower layer whose thickness depends on the fractional ice coverage for each grid box and the number of months with some ice present. The total ice thickness also may be augmented by snow accumulation (see Snow Cover). The temperature profile in each ice layer is assumed to be a quadratic function of depth, with coefficients that are solved subject to six constraints. (These include consistency conditions on the temperatures and heat fluxes at the interfaces between the atmosphere and the ice, between the ice layers, and between the ice and the ocean; in addition, the mean temperature of each ice layer is set equal to its value from the previous hour.) After the ice temperature profiles are determined, the heat fluxes at the atmosphere-ice, ice-ice, and ice-ocean interfaces are updated. See also Surface Fluxes. 


\section{Snow Cover}

Precipitation falls as snow if the temperature of the first vertical layer above the surface is $<0^{\circ} \mathrm{C}$. Snow may accumulate on land, on continental ice, and on sea ice up to $0.1 \mathrm{~m}$ equivalent water, after which it augments the ice thickness (see Sea Ice). Snow depth is computed prognostically as the balance of snowfall, snowmelt, and sublimation (which contributes to the surface evaporative flux--see Surface Fluxes). Net heating of the snow surface raises the ground temperature to $0^{\circ} \mathrm{C}$; additional heating produces snowmelt, which contributes to soil moisture and affects the thermal properties of the soil (see Land Surface Processes). Snow also modifies the surface albedo (see Surface Characteristics).

\section{Surface Characteristics}

- Each grid box is assigned appropriate fractions of land and ocean, and part of the ocean fraction may be covered by ice (see Sea Ice). In addition, permanent ice sheets are specified for Antarctica, Greenland, and some Arctic islands. Vegetation type is a composite over each grid-box from 32 classifications distinguished in the $1 \times 1$-degree data of Matthews (1983, 1984).

- Over land, surface roughness is a fit to the data of Fiedler and Panofsky (1972) as a function of the standard deviation of the orography (see Orography). The maximum of this roughness and that of the local vegetation (including a "zero plane displacement" value for tall vegetation types--cf. Monteith 1973) determines the roughness over land. Over sea ice, the roughness length is a uniform $4.3 \times 10^{-4} \mathrm{~m}$, after Doronin (1969). Over ocean, the surface roughness is a function of the momentum flux and is used to compute the neutral drag coefficient as well as the Stanton and Dalton numbers (see Surface Fluxes).

- The surface albedo is prescribed for ocean, land ice, sea ice, and for eight different land surface types after the data of Matthews $(1983,1984)$. The visible and near infrared albedos are distinguished, and seasonal variations in the albedo for vegetated surfaces are included. The albedo of snow-covered ground is the snow-free value modified by factors depending on snow depth, snow age, and vegetation masking depth, which varies with vegetation type. Ocean albedo is a function of surface wind speed and solar zenith angle after Cox and Munk (1956).

- The spectral dependence of longwave emissivity for deserts is included from 


\section{GISS}

data of Hovis and Callahan (1966), and for snow and ice from data of Wiscombe and Warren (1980). The emissivity of the ocean is a function of the surface wind speed and of the albedo.

\section{Surface Fluxes}

- The absorbed surface solar flux is determined from albedos, and the surface longwave emission from the Planck function with spatially variable emissivities (see Surface Characteristics).

- The surface wind stress is expressed as a product of air density, a drag coefficient, and the surface wind speed and velocity (see Planetary Boundary Layer). The surface drag coefficient is a function of both roughness length (see Surface Characteristics) and vertical stability.

- Over land, the latent heat flux is computed separately for bare and vegetated surfaces (see Land Surface Processes), following Penman (1948) and Monteith (1981). Over ocean and ice surfaces, the latent heat flux is expressed by a bulk formula that includes the product of the surface air density, a transfer coefficient, the surface wind speed, and the difference between the saturation value of mixing ratio at the ground temperature and the surface atmospheric value (see Planetary Boundary Layer).

- Over land, the sensible heat flux is determined as the residual of the total net heat flux computed by the surface model (see Land Surface Processes) minus the latent heat flux. (The partitioning of the latent and sensible heat fluxes, or Bowen ratio, implies the surface temperature--see Land Surface Processes.) Over ocean and ice surfaces, the sensible heat flux is calculated from a bulk aerodynamic formula as a product of the surface air density and heat capacity, a transfer coefficient, a surface wind speed, and the difference between the skin temperature and the surface air temperature (see Planetary Boundary Layer). (The transfer coefficient is a stabilitydependent function of the drag coefficient and is different from that used for the latent heat flux over ocean and ice.)

\section{Land Surface Processes}

- Soil temperature is computed by solving a heat diffusion equation in six layers. The thickness of the top layer varies, but is approximately $0.1 \mathrm{~m}$. The thicknesses of deeper layers increase geometrically, with the bottom boundary of the soil column at 
a nominal bedrock depth of $3.444 \mathrm{~m}$. The upper boundary condition is the balance of surface energy fluxes (see Surface Fluxes); at the bottom boundary, zero net heat flux is specified. The thermal conductivity and heat capacity of the ground vary with snow cover, as well as soil moisture amount and phase.

- Land-surface hydrology is treated after the physically based model of Abramopoulos et al. (1988). The scheme includes a vegetation canopy, a composite over each grid box from the vegetation types of Matthews $(1983,1984)$, that intercepts precipitation and dew. Evaporation from the wet canopy and from bare soil is treated, as well as soil-moisture loss from transpiration according to moisture availability and variable vegetation resistance and root density. Diffusion of moisture is predicted in the six soil layers, accounting for spatially variable composite conductivities and matric potentials that depend on soil type and moisture content. Infiltration of precipitation and snowmelt is explicitly calculated, with surface runoff occurring when the uppermost soil layer is saturated; underground runoff that depends on topographic slope is also included. 


\section{GLA: Goddard Laboratory for Atmospheres}

AMIP Representative(s)

Dr. William K.-M. Lau, Goddard Laboratory for Atmospheres, Mail Code 913, Goddard Space Flight Center, Greenbelt, Maryland, 20771; Phone: +1-301-286-7208; Fax: +1-301-286-1759; e-mail: lau@climate.gsfc.nasa.gov.

\section{Model Designation}

GLA GCM-01.0 AMIP-01 4x5L17

\section{Model Lineage}

The GLA model is derived from an earlier version described by Kalnay et al. (1983). Modifications include increased vertical resolution and several changes in the parameterizations of radiation, convection, cloud formation, precipitation, vertical diffusion, and surface processes (cf. Sud and Walker 1992). Although both the GLA model and the GSFC/GEOS-1 model (for example, cf. Schubert et al. 1993) are in use at the Goddard Laboratory for Atmospheres, they differ substantially in their dynamical formulations and numerics, as well as in their physical parameterizations, especially those pertaining to convection and land surface processes.

\section{Model Documentation}

Documentation of different aspects of the model is provided by Kalnay et al. (1983), Harshvardhan et al. (1987), Helfand and Labraga (1988), Helfand et al. (1991), Sellers et al. (1986), Sud and Molod (1986, 1988), Sud et al. (1991, 1992), Sud and Walker (1992, 1993), and Xue et al. (1991).

\section{Numerical/Computational Properties}

\section{Horizontal Representation}

Finite differences on an energy- and momentum-conserving A-grid (cf. Arakawa and Lamb 1977). The horizontal advection of atmospheric variables is accurate to fourth-order (cf. Kalnay et al. 1983). 


\section{Horizontal Resolution}

$4 \times 5$-degree latitude-longitude grid.

\section{Vertical Domain}

Surface to about $12 \mathrm{hPa}$. For a surface pressure of $1000 \mathrm{hPa}$, the lowest atmospheric level is at a pressure of about $994 \mathrm{hPa}$.

\section{Vertical Representation}

Finite-differences in sigma coordinates. The vertical differencing scheme conserves squared potential temperature (cf. Arakawa and Suarez 1983).

\section{Vertical Resolution}

There are 17 unequally spaced sigma levels. For a surface pressure of $1000 \mathrm{hPa}$, 5 levels are below $800 \mathrm{hPa}$ and 4 levels are above $200 \mathrm{hPa}$.

\section{Computer/Operating System}

The AMIP simulation was run on a Cray Y/MP computer using a single processor in the UNICOS environment.

\section{Computational Performance}

For the AMIP experiment, about 6 minutes of Cray Y/MP computer time per simulated day.

\section{Initialization}

For the AMIP simulation, the model atmospheric state was initialized from the ECMWF analysis for $00 \mathrm{Z}$ on 1 January 1979. Soil moisture and snow cover/depth were initialized from a previous model solution.

\section{Time Integration Scheme(s)}

Time integration is carried out with a Matsuno step at the start, and with leapfrog steps thereafter. The time step for dynamics is 3.75 minutes. Most model physics, including shortwave radiation, moist convection, large-scale condensation, evaporation of precipitation, cloud formation and properties, and surface processes are 
calculated every 30 minutes; longwave radiation is computed hourly. In addition, the level 2.5 turbulence closure scheme (see Diffusion) uses an implicit backward operator with 5-minute time step to determine the turbulence kinetic energy (TKE); however, the rate of production of TKE and the diffusion coefficients are calculated by a forward time step.

\section{Smoothing / Filling}

Orography is smoothed (see Orography). At every dynamical time step (see Time Integration Schemes), a sixteenth-order Shapiro (1970) filter (with time scale 90 minutes) is applied to the prognostic fields; a Fourier filter is also applied in polar latitudes. Negative moisture values are filled by "borrowing" moisture from the level below, and from neighboring horizontal grid boxes at the lowest vertical level.

\section{Sampling Frequency}

For the AMIP simulation, the model history is written every 6 hours.

\section{Dynamical/Physical Properties}

\section{Atmospheric Dynamics}

Primitive-equation dynamics in flux form are expressed in terms of $\mathrm{u}$ and $\mathrm{v}$ winds, temperature, specific humidity, and surface pressure.

\section{Diffusion}

- Horizontal diffusion is not included.

- The effects of vertical diffusion are treated by the level-2.5 second-order turbulence closure model of Helfand and Labraga (1988). TKE is a prognostic variable, and the remaining turbulent second moments (including vertical fluxes) are diagnostically determined. See also Planetary Boundary Layer, Surface Fluxes, and Time Integration Schemes.

\section{Gravity-wave Drag \\ Gravity-wave drag is not modeled.}




\section{Solar Constant/Cycles}

The solar constant is the AMIP-prescribed value of $1365 \mathrm{~W} \mathrm{~m}^{-2}$. Both seasonal and diurnal cycles in solar forcing are simulated.

\section{Chemistry}

The carbon dioxide concentration is the AMIP-prescribed value of $345 \mathrm{ppm}$. Monthly climatological zonal profiles of ozone concentrations are prescribed (cf. Rosenfield et al. 1987). Radiative effects of water vapor and of a single type of aerosol, which is present at constant global concentration in the model's planetary boundary layer (PBL), also are included. See also Planetary Boundary Layer and Radiation.

\section{Radiation}

- Atmospheric radiation is treated as in Harshvardhan et al. (1987). The shortwave parameterization after Davies (1982) follows the approach of Lacis and Hansen (1974) for clear-sky Rayleigh scattering, and for ozone absorption in the ultraviolet (wavelengths $<0.35$ micron) and visible (wavelengths 0.5 to 0.7 micron) spectral bands. Water vapor absorption in the near-infrared ( 0.7 to 4.0 microns) is treated as in Chou (1986).

- In the visible spectral band, scattering by clouds and scattering/absorption by aerosol (see Chemistry) is treated by a delta-Eddington approximation (cf. Joseph et al. 1976). Optical properties for a single aerosol type are specified from global climatological data (cf. Deepak and Gerber 1983 and Sud and Walker 1992). For clouds, multiple-scattering effects are computed following Lacis and Hansen (1974), but the cloud optical thickness (which also depends on cloud type, specific humidity, and solar zenith angle) is adjusted according to the local fraction of cloud cover (cf. Sud and Walker 1992). Cloud optical thicknesses are set about 2.5 times higher than the Lacis and Hansen values, in agreement with values derived by Peng et al. (1982) from Feigelson (1978) aircraft measurements. For cumulus clouds, an optical thickness of 20 is assumed for all sigma layers that have detraining anvil clouds (see Cloud Formation). Values of single-scattering albedo and of asymmetry factor which are also required for the delta-Eddington approximation, are prescribed.

- For longwave absorption, the broadband transmission approach of Chou (1984) is used for water vapor, that of Chou and Peng (1983) for carbon dioxide, and that of 
Rodgers (1968) and Rosenfield et al. (1987) for ozone. Longwave absorption is calculated in five spectral bands (for wavenumbers between 0 and $3 \times 10^{5} \mathrm{~m}^{-1}$ ), with continuum absorption by water vapor treated as in Roberts et al. (1976). The emissivities of clouds depend on their optical thicknesses, and fractional cloud cover effects are implemented by area-weighted, cloud-radiative interactions. For purposes of the radiation calculations, all clouds are assumed to be randomly overlapped in the vertical. See also Cloud Formation.

\section{Convection}

- The formulation of convection follows the scheme of Arakawa and Schubert (1974), as implemented in discrete form by Lord and Arakawa (1980). Changes made to the minimum entrainment rate and to the critical cloud work function are the only modifications of the original scheme (cf. Sud and Molod 1988, Tokioka et al. 1988, Sud et al. 1991, and Sud and Wallier 1992).

- A relative humidity of at least 90 percent is required at the cloud base for the onset of convection. The scheme predicts mass fluxes from mutually interacting cumulus subensembles which have different entrainment rates and levels of neutral buoyancy that define the tops of the clouds and their associated convective updrafts; a full spectrum of cumulus clouds of depths compatible with the vertical discretization (see Vertical Resolution) is included. The mass fluxes, which are assumed to originate in the PBL (see Planetary Boundary Layer), are optimal solutions of an integral equation, subject to a quasi-equilibrium constraint (cf. Lord et al. 1982); these solutions are obtained by a simplex numerical method.

- In turn, the predicted convective mass fluxes feed back on the large-scale fields of temperature (through latent heating and compensating subsidence), moisture (through precipitation and its evaporation, detrainment, and subsidence), and momentum (through cumulus friction). The effects on convective cloud buoyancy of phase changes from water to ice also are treated, but the drying and cooling effects of convective-scale downdrafts on the environment are not explicitly parameterized. (However, these can occur implicitly via turbulent fluxes.)

- Convective instability originating above the model's PBL is treated within the Helfand and Lebraga (1988) turbulence scheme (see Diffusion). For unstable conditions in which the virtual potential temperature exceeds that of the layer above, an 
eddy exchange coefficient of high magnitude (100 or more) is assigned to bring about a dry convective adjustment.

\section{Cloud Formation}

- Subgrid-scale cloud forms above the PBL as part of the cumulus convection scheme (see Convection). In the process of producing convective rain, the scheme also determines the mass of associated detraining anvil cloud. The anvils are assumed to have a conical shape and to be equal to the sigma-layer thickness at their center, while linearly tapering off to zero at the cone edge (facilitating determination of fractional cloudiness for calculation of cloud-radiative interactions--see Radiation).

- Large-scale precipitating cloud forms if there is local supersaturation after the model is adjusted by the convective clouds. In that case, 80 percent of a grid box is assumed to be filled by cloud (this also impacts the large-scale rainfall fraction and intensity--see Precipitation).

- Large-scale nonprecipitating cloud also may form; its fractional coverage is determined after Slingo and Ritter (1985) as a quadratic function of the difference between the local relative humidity of a layer and a threshold value that is specified as a nonlinear function of sigma level (cf. Sud and Walker 1992).

\section{Precipitation}

- Convective precipitation forms as part of the cumulus convection scheme and/ or related moist convective adjustments (see Convection). Large-scale precipitation forms under supersaturated conditions.

- Both large-scale and convective precipitation evaporate in falling to the surface; the former is taken to be spatially homogeneous (see Cloud Formation), while the latter is assumed to follow the satellite-derived intensity distribution function of Ruprecht and Gray (1976). The evaporation parameterization accounts for precipitation intensity, drop size distribution, sphericity influence of hydrometeors, and the temperature, pressure, and relative humidity of the ambient air (cf. Sud and Molod 1988 and Sud and Walker 1993). 


\section{Planetary Boundary Layer}

The PBL is defined by those layers with significant TKE; for practical purposes, the PBL is represented by the first 3 levels above the surface (at sigma $=0.994,0.971$, and 0.930). Near the surface, the PBL is treated as an extended surface layer and a viscous sublayer in the space between the surface and the tops of the surface roughness elements. Appropriate parameterizations are utilized to determine turbulent fluxes in the different PBL subregions. See also Diffusion, Surface Characteristics, and Surface Fluxes.

\section{Orography}

The $1 \times 1$-degree topographic height data of Gates and Nelson (1975) is areaaveraged over the $4 \times 5$-degree grid boxes. The resulting orography is smoothed using a sixteenth-order Shapiro (1970) filter, and a Fourier filter poleward of 60 degrees latitude. Negative terrain heights resulting from the smoothing process are set to zero.

\section{Ocean}

AMIP monthly sea surface temperature fields are prescribed, with daily values determined by linear interpolation.

Sea Ice

AMIP monthly sea ice extents are prescribed. Snow is allowed to accumulate, but without conversion to ice (the ice thickness is kept a uniform 3 meters). The ice surface temperature is predicted from the net flux of energy (see Surface Fluxes), including a conduction heat flux which is proportional to the difference between the ice surface temperature and that prescribed $\left(-2^{\circ} \mathrm{C}\right)$ for the ocean below.

\section{Snow Cover}

Precipitation falls as snow if the surface air temperature is $<0{ }^{\circ} \mathrm{C}$. Snow may accumulate only on land, and fractional snow coverage of a grid box is parameterized as in the Simple Biosphere ( $\mathrm{SiB}$ ) model of Sellers et al. (1986). Snow mass is a prognostic variable that is depleted by both sublimation (which contributes to surface evaporation) and snowmelt (which contributes to soil moisture). Snow cover alters both the albedo and the thermal properties of the surface. See also Surface Characteristics, Surface Fluxes, and Land Surface Processes. 


\section{Surface Characteristics}

- Over ice surfaces, the roughness length is prescribed as a uniform $1 \times 10^{-4} \mathrm{~m}$. The roughness of the ocean is computed iteratively as a function of the surface wind stress which is an interpolation between the relation for moderate to high winds given by Large and Pond (1981), and by Kondo (1975) for the range of weak winds. Over land, the 12 vegetation/surface types of the SiB model of Sellers et al. (1986) and associated roughness lengths are specified at monthly intervals (cf. Dorman and Sellers 1989).

- Surface albedos depend on solar zenith angle (cf. Pinker and Laszlo 1992) and include values for both visible (0.0-0.7 micron) and near-infrared (0.7-4.0 microns) spectral intervals. Monthly varying surface albedos for vegetated land are specified from the $\mathrm{SiB}$ model. Albedos of bare land, ocean, and ice are prescribed from Earth Radiation Budget Experiment (ERBE) satellite data (cf. Barkstrom et al. 1990). Snow cover alters the land albedos, as described by Xue et al. (1991).

- Over vegetated surfaces, longwave emissivity may be less than unity (graybody emission), but is 1.0 otherwise (blackbody emission).

\section{Surface Fluxes}

- Surface solar absorption is determined from albedos, and longwave emission from the Planck equation with prescribed emissivities (see Surface Characteristics).

- Turbulent eddy fluxes of momentum, heat, and moisture are parameterized according to surface type and vertical location within the PBL (see Planetary Boundary Layer). Over ocean and ice surfaces, Monin-Obukhov similarity functions expressed as stability-dependent bulk formulae represent the vertical structure of an extended surface layer (of depth up to $150 \mathrm{~m}$ ). Near-surface values of wind, temperature, and humidity required for the bulk formulae are taken to be those at the lowest atmospheric level (sigma $=0.994$ ). For an unstable surface layer, the stability function is the KEYPS function of Panofsky (1973) for momentum and its generalization for heat and moisture (which assures nonvanishing heat and moisture fluxes even for zero surface wind speed). For a stable surface layer, the stability functions are those of Clarke (1970) for heat and moisture fluxes, and a slightly modified form for the surface momentum flux. The gradients in temperature and moisture in the laminar sublayer (between the surface and the tops of the roughness 
elements) are based on the relation of Yaglom and Kader (1974). Cf. Helfand (1985) and Helfand et al. (1991) for further details.

- Over land, turbulent surface fluxes are represented by bulk formulae following the formulation of Deardorff (1972), but with stability-dependent drag and transfer coefficients (expressed as aerodynamic and surface resistances) that are determined as in Xue et al. (1991). The surface moisture flux includes direct evaporation from a vegetation canopy and from bare soil, as well as evapotranspiration via root uptake (see Land Surface Processes).

- Above the extended surface layer, turbulent fluxes of momentum, heat, and moisture are predicted by the Helfand and Labraga (1988) level-2.5 second-order turbulence closure scheme (see Diffusion).

\section{Land Surface Processes}

- Land-surface processes are simulated as in the Xue et al. (1991) modification of the $\mathrm{SiB}$ model of Sellers et al. (1986). Within the single-story vegetation canopy, evapotranspiration from dry leaves includes detailed modeling of stomatal and canopy resistances; direct evaporation from both bare soil and from the canopy interception of precipitation is also treated (see Surface Fluxes).

- Soil temperature is determined in 2 layers by the force-restore method of Deardorff (1978). Soil moisture, which is predicted from budget equations in 3 layers, is increased by infiltrated precipitation and snowmelt, and is depleted by evapotranspiration and direct evaporation. Infiltration of moisture is limited by the hydraulic conductivity of the soil, with the remainder contributing to surface runoff. Deep runoff from gravitational drainage is also simulated. See also Surface Characteristics and Surface Fluxes. 


\section{GSFC: Goddard Space Flight Center}

\section{AMIP Representative(s)}

Dr. Chung-Kyu Park and Dr. Richard Rood, Goddard Laboratory for Atmospheres, Mail Code 910.3, Goddard Space Flight Center, Greenbelt, Maryland 20771; Phone: +1-301-286-8695; Fax: +1-301-286-1754; e-mail: park@ckpark.gsfc.nasa.gov (Park) and rood@sgccp.gsfc.nasa.gov (Rood).

\section{Model Designation}

GSFC GEOS-1 4x5L20

\section{Model Lineage}

The GSFC model, equivalent to the Goddard Earth Observing System-1 (GEOS-1) model, was developed by the Data Assimilation Office of the Goddard Laboratory for Atmospheres (GLA). The GSFC/GEOS-1 model is designed for use with an optimal interpolation analysis scheme for production of multi-year global atmospheric datasets (cf. Schubert et al. 1993). The earliest model predecessor was based on the "plugcompatible" concepts outlined by Kalnay et al. (1989), and subsequent refinements are described by Fox-Rabinovitz et al. (1991), Helfand et al. (1991), and Suarez and Takacs (1993). The GSFC/GEOS-1 model represents a different historical line of development from that of the GLA model (cf., for example, Sud and Walker 1992), which is also in use at the Goddard Laboratory for Atmospheres. The GSFC/GEOS-1 and GLA models differ substantially, especially in their dynamical formulations and numerics, as well as in physical parameterizations pertaining to the treatment of convection and land surface processes.

\section{Model Documentation}

A summary of basic model features is provided by Schubert et al. (1993). Details of the numerics are given by Suarez and Takacs (1993). The radiation scheme is that of Harshvardhan et al. (1987). The parameterizations of convection and evaporation of rainfall follow Moorthi and Suarez (1992) and Sud and Molod (1988) respectively. Treatment of turbulent dissipation is based on formulations of Helfand and Labraga (1988) and Helfand et al. (1991). 


\section{GSFC}

\section{Numerical/Computational Properties}

Horizontal Representation

Finite differences on a staggered Arakawa C-grid that c:nserves potential enstrophy and energy (cf. Burridge and Haseler 1977).

Horizontal Resolution

$4 \times$ 5-degree latitude-longitude grid.

\section{Vertical Domain}

Surface to about $10 \mathrm{hPa}$. For a surface pressure of $1000 \mathrm{hPa}$, the lowest atmospheric level is at a pressure of about $994 \mathrm{hPa}$.

\section{Vertical Representation}

Unstaggered finite-differences in generalized sigma coordinates. Th vertical differencing scheme is that of Arakawa and Suarez (1983), which conserves t e global mass integral of potential temperature for adiabatic processes, and ensures $i$ accurate finite-difference analogue of the energy-conversion term and the pressure radient force.

\section{Vertical Resolution}

There are 20 unevenly spaced sigma levels. For a surface pressure of $1000 \mathrm{hPc}$ 5 levels are below $800 \mathrm{hPa}$ and 7 levels are above $200 \mathrm{hPa}$.

\section{Computer/Operating System}

The AMIP simulation was run on a Cray Y/MP computer using a single processor in the UNICOS environment.

\section{Computational Performance}

For the AMIP experiment, about 4 minutes of Cray Y/MP computer time per simulated day. 


\section{Initialization}

For the AMIP simulation, the model atmospheric state is initialized for 1 January 1979 from the ECMWF reanalysis of the FGGE period. The initial soil wetness fractions (see Land Surface Processes) are specified from the January 1979 estimates of Schemm et al. (1992), and snow cover from a January climatology (see Snow Cover).

\section{Time Integration Scheme(s)}

The main time integration is by a leapfrog scheme with an Asselin (1972) time filter. Turbulent surface fluxes and vertical diffusion (see Diffusion and Surface Fluxes) are computed by a backward-implicit iterative time scheme. The time step for dynamics is 5 minutes. To avoid introducing shocks and imbalances in the dynamics, diabatic increments are added at each dynamical time step. The tendencies of diabatic processes are updated at time steps of 10 minutes for moist convection, 30 minutes for turbulent dissipation, and 3 hours for radiative fluxes.

\section{Smoothing / Filling}

- Orography is smoothed (see Orography). A sixteenth-order Shapiro (1970) filter is applied to the winds, potential temperature, and specific humidity in order to damp small-scale dispersive waves. (The filter is applied fractionally at every 5-minute dynamical time step such that the amplitude of the two-grid interval wave is reduced by half in two hours.) A high-latitude Fourier filter also is used to avoid violation of the Courant-Friedrichs-Lewy (CFL) condition for the Lamb wave and internal gravity waves. This polar filter is applied only to the tendencies of the winds, potential temperature, specific humidity, and surface pressure.

- Negative values of specific humidity in a vertical column are filled by borrowing from below, with negative moisture points in the lowest layer set to zero.

\section{Sampling Frequency}

For the AMIP simulation, the history of prognostic atmospheric variables is produced every 6 hours at 18 pressure levels, while surface and vertically integrated diagnostics are generated every 3 hours. 


\section{GSFC}

\section{Dynamical/Physical Properties}

\section{Atmospheric Dynamics}

Primitive-equation dynamics are expressed in terms of $u-v$ winds, potential temperature, specific humidity, and surface pressure. The momentum equations are written in "vector-invariant" form (cf. Sadourny 1975b and Arakawa and Lamb 1981), while the thermodynamic and moisture equations are rendered in flux form to facilitate conservation of potential temperature and specific humidity.

\section{Diffusion}

- Horizontal diffusion is not modeled.

- Above the surface layer (see Surface Fluxes), turbulent fluxes of momentum, heat, and moisture are calculated by the level-2.5 closure scheme of Helfand and Labraga (1988), which predicts turbulence kinetic energy (TKE) and determines the eddy transfer coefficients used for a bulk formulation.

\section{Gravity-wave Drag}

Gravity-wave drag is not modeled.

\section{Solar Constant/Cycles}

The solar constant is the AMIP-prescribed value of $1365 \mathrm{~W} \mathrm{~m}^{-2}$. Both seasonal and diurnal cycles in solar forcing are simulated.

\section{Chemistry}

The carbon dioxide concentration is the AMIP-prescribed value of $345 \mathrm{ppm}$. Monthly zonal profiles of ozone concentrations are specified from data of Rosenfield et al. (1987), with linear interpolation for intermediate time steps. Radiative effects of water vapor, but not those of aerosols, are also included (see Radiation).

\section{Radiation}

- Atmospheric radiation is simulated by the scheme of Harshvardhan et al. (1987). The shortwave parameterization after Davies (1982) follows the approach of Lacis and Hansen (1974). Absorption by water vapor in near-infrared (0.70 to 4.0 microns) 
spectral ranges, and by ozone in the visible ( 0.45 to 0.75 micron) and ultraviolet ( 0.24 to 0.36 micron) is treated.

- The parameterization of longwave radiation employs a wide-band model, with four broad-band transmissions. Water vapor absorption in two bands centered at 9.6 and 15 microns is calculated after the method of Chou (1984), based on both line-type and e-type approximations. Absorption by carbon dioxide follows the scheme of Chou and Peng (1983), which separates the band-wing and band-center scaled paths. Absorption by ozone applies the Rosenfield et al. (1987) modifications of the Rodgers (1968) line width.

- Shortwave scattering by clouds (as a function of solar zenith angle) is treated by the Meador and Weaver (1980) modified delta-Eddington approximation; cloud albedo and transmissivity are obtained from specified single-scattering albedo and optical thickness. Cloud-water absorption is determined from a multiple-scattering computation with k-distribution functions. In the longwave, all clouds act as blackbodies (emissivity $=1.0$ ). The cloud fractions produced by moist convective processes (see Convection) are used to evaluate clear line-of-site probabilities and effective optical thicknesses. For purposes of the radiation calculations, deep convective cloud is fully overlapped in the vertical, while shallow convective and nonconvective clouds are randomly overlapped. See also Cloud Formation.

\section{Convection}

- Penetrative and shallow cumulus convection are simulated by the Relaxed Arakawa-Schubert (RAS) scheme of Moorthi and Suarez (1992), a modification of the Arakawa and Schubert (1974) parameterization. The RAS scheme predicts mass fluxes from convective cloud types that have different entrainment rates and levels of neutral buoyancy. The predicted convective mass fluxes are used to solve budget equations that determine the impact of convection on the grid-scale fields of temperature (through latent heating and compensating subsidence) and moisture (through precipitation and detrainment).

- The mass flux for each cloud type in RAS is predicted from an equation for the cloud work function, defined as the tendency of cumulus kinetic energy (CKE). The equation is solved by assuming that the rate of generation of CKE by the large-scale environment is balanced by dissipation at the scale of the cumulus subensemble (that is, a quasi-equilibrium condition). To approximate full interaction between the 
different cloud types, many clouds are simulated frequently; each modifies the environment by some fraction of the total adjustment, with a relaxation towards neutrality. See also Cloud Formation and Precipitation.

\section{Cloud Formation}

- Convective cloud is determined diagnostically as part of the RAS scheme (see Convection). The lowest two model layers are regarded as a single subcloud layer (nominally $50 \mathrm{hPa}$ thick); then, if detrainment occurs in the next two higher layers when the RAS scheme is invoked (every 10 minutes of the integration), the convection is defined as shallow with randomly overlapped cloud, and a fractional cloudiness of 0.5 is assigned at the detrainment level. In addition, 10 other cloud-top levels are randomly chosen between cloud base and the top layer; if deep convection with a cloud-top pressure $<400 \mathrm{hPa}$ occurs, the associated cloud is treated as fully overlapped with a fractional cloudiness of unity at the detrainment level.

- Large-scale randomly overlapped cloud is prescribed when grid-scale supersaturation occurs in the absence of deep convective cloud (to ensure that total cloud fraction does not exceed unity). Under such conditions, the grid box is assumed to be instantaneously covered with the large-scale cloud (cloudiness fraction of 1 ). See also Radiation for cloud-radiative interactions.

\section{Precipitation}

- Convective precipitation results from operation of the cumulus convection scheme (see Convection). Large-scale precipitation forms under supersaturated conditions (see Cloud Formation).

- Both large-scale and convective precipitation may evaporate in falling to the surface (cf. Sud and Molod 1988). The evaporation parameterization takes into account rainfall intensity, drop size distribution, and the temperature, pressure, and relative humidity of the aribient air; the moisture deficit of a layer is treated as a free parameter.

\section{Planetary Boundary Layer}

The PBL height is diagnosed as the level at which TKE is reduced to 10 percent of its surface value (see Diffusion), typically within the first 2 to 4 levels above the surface (sigma $=0.994$ to 0.875). See also Surface Characteristics and Surface Fluxes. 


\section{Orography}

Surface orography is determined from area-averaging the U.S. Navy topographic height data with 10-minute arc resolution (cf. Joseph 1980) over the model's 4 x 5-degree grid. The resulting heights are passed through a Lanczos (1966) filter to remove the smallest scales, and negative values are refilled.

\section{Ocean}

AMIP monthly sea surface temperature fields are prescribed, with linear interpolation to intermediate time steps (see Time Integration Schemes).

Sea Ice

AMIP monthly sea ice extents are prescribed and linearly interpolated to intermediate time steps. The ice is assumed to have a uniform thickness of $3 \mathrm{~m}$, and the heat conduction through it is accounted for as part of the surface energy budget (see Surface Fluxes), with the surface temperature over ice determined prognostically. Snow is not present on sea ice (see Snow Cover).

\section{Snow Cover}

Snow mass is not a prognostic variable. Monthly snow cover over land is prescribed from satellite-derived surface albedo estimates of Matson (1978): wherever the albedo of a grid box exceeds 0.40 , that area is defined as snowcovered. (In the Southern Hemisphere, snow cover is only specified for Antarctica). If precipitation falls when the ground temperature is $<0^{\circ} \mathrm{C}$, some thermodynamic effects of snow cover are also included. See also Surface Characteristics and Land Surface Processes.

\section{Surface Characteristics}

- Over land, monthly varying roughness lengths are specified from the data of Dorman and Sellers (1989). A uniform roughness of $1 \times 10^{-4} \mathrm{~m}$ is prescribed for ice surfaces. Over the oceans, the roughness is computed as an interpolation between the functions of Large and Pond (1981) for high surface winds and of Kondo (1975) for weak winds.

- The surface albedo is specified as a uniform 0.80 over ice surfaces, but over the 


\section{GSFC}

oceans it is a function of solar zenith angle. Monthly varying surface albedos of snowfree land are specified following modified Posey and Clapp (1964) data. The albedos of snow-covered land (see Snow Cover) are specified from monthly satellitederived estimates of Matson (1978), and depend on the surface type (with seven types distinguished), but not spectral interval. Monthly albedos are linearly interpolated to intermediate time steps.Cf. Kitzmiller (1979) for further details.

- Longwave emissivity is assumed to be unity (blackbody emission) for all surfaces.

\section{Surface Fluxes}

- Surface solar absorption is determined from albedos, and longwave emission from the Planck equation with prescribed emissivity of 1.0 (see Surface Characteristics).

- Turbulent eddy fluxes of momentum, heat, and moisture in the extended surface layer are calculated from stability-dependent bulk formulae based on Monin-Obukhov similarity functions. For an unstable surface layer, the chosen stability functions are the KEYPS function for the momentum flux (cf. Panofsky 1973) and its generalization for heat and moisture (which assures nonvanishing fluxes as the surface wind speed approaches zero). For a stable surface layer, the stability functions are those of Clarke (1970), but they are slightly modified for the momentum flux. The vertical gradients in temperature and moisture are based on the relation of Yaglom and Kader (1974). The surface moisture flux also depends on the evapotranspiration efficiency $\beta$, which is specified as unity over oceans, but which over land is given by the locally prescribed monthly soil wetness fraction (see Land Surface Processes). Cf. Helfand (1985) and Helfand et al. (1991) for further details. See also Diffusion.

\section{Land Surface Processes}

- Soil temperature is determined from a surface energy balance (see Surface Fluxes), excluding provision for subsurface heat storage. When precipitation falls on ground with temperature $<0{ }^{\circ} \mathrm{C}$, the conductance of the soil is modified to partially account for the thermodynamic effects of snow (see Snow Cover).

- The spatially variable soil wetness fraction (ratio of local soil moisture content 
to a uniform field capacity of $0.15 \mathrm{~m}$ of water) is prescribed from monthly estimates of Schemm et al. (1992). These are based on the procedure developed by Mintz and Serafini (1984) using a single-layer "bucket" model in conjunction with monthly observed surface air temperature and precipitation for the AMIP period 1979 to 1988. 


\section{IAP: Institute of Atmospheric Physics (of the Chinese Academy of Sciences)}

\section{AMIP Representative(s)}

Dr. Qing-cun Zeng, Laboratory of Numerical Modelling for Atmospheric Sciences and Geophysical Fluid Dynamics, Institute of Atmospheric Physics, Chinese Academy of Sciences, P.O. Box 2718, Beijing 100080, China; Phone: +86-1-2562347; Fax: +86-1-2562347

\section{Model Designation}

\section{IAP IAP-2L $4 \times 5 \mathrm{~L} 2$}

\section{Model Lineage}

The IAP model consists of a special dynamical framework developed by Zeng and Zhang (1987) and Zeng et al. (1987) combined with physics similar to that of the Oregon State University model described by Ghan et al. (1982).

\section{Model Documentation}

The principal documentation of the IAP model is provided by Zeng et al. (1989).

\section{Numerical/Computational Properties}

Horizontal Representation

The available-energy conserving, finite-difference scheme of Zeng and Zhang (1982) and Zeng et al. (1987) is applied on a staggered C-grid (cf. Arakawa and Lamb 1977).

\section{Horizontal Resolution}

$4 \times 5$-degree latitude-longitude grid.

\section{Vertical Domain}

Surface to $200 \mathrm{hPa}$ for dynamics (with the highest prognostic level at $400 \mathrm{hPa}$ ). For a surface pressure of $1000 \mathrm{hPa}$, the first atmospheric level is at $800 \mathrm{hPa}$. See also Vertical Representation and Vertical Resolution. 


\section{Vertical Representation}

Finite differences in modified sigma coordinates: sigma $=(\mathrm{P}-\mathrm{PT}) /(\mathrm{PS}-\mathrm{PT})$, where $\mathrm{P}$ is atmospheric pressure, $\mathrm{PT}$ is $200 \mathrm{hPa}$ (the dynamical top of the model), and PS is the surface pressure.

\section{Vertical Resolution}

There are 2 equally spaced modified sigma levels (see Vertical Representation). For a surface pressure of $1000 \mathrm{hPa}$, these are at $800 \mathrm{hPa}$ and $400 \mathrm{hPa}$ (with the dynamical top at $200 \mathrm{hPa}$ ).

\section{Computer/Operating System}

The AMIP simulation was run on a Convex-C120 computer using a single processor.

\section{Computational Performance}

For the AMIP experiment, about 5 minutes of Convex-C120 time per simulated day.

\section{Initialization}

For the AMIP experiment, the initial conditions for the atmosphere, soil moisture, and snow cover/depth are obtained from a model simulation of perpetual January using the AMIP-prescribed ocean temperatures and sea ice extents for 1 January 1979. See also Ocean and Sea Ice.

\section{Time Integration Scheme(s)}

The model uses a leapfrog scheme, followed by time filtering to damp the computational mode (cf. Robert 1966). The pressure gradient force terms are also smoothed (cf. Schuman 1971) to permit use of a longer time step, which is 6 minutes for dynamics, 30 minutes for diffusion, and 1 hour for physics (including radiation). The vertical flux of atmospheric moisture is also computed hourly, and it is recomputed if conditional instability of a computational kind occurs (cf. Arakawa 1972), as evidenced by relative humidities in excess of 100 percent. 


\section{Smoothing / Filling}

- Orography is smoothed (see Orography). Poleward of 70 degrees latitude, the wave-selected damping technique of Arakawa and Lamb (1977) and the Fast Fourier Transform algorithm of $\mathrm{Lu}$ (1986) are applied; from 38 to 70 degrees latitude, the recursive operator of Fjortoft (1953) is also used. In addition, a Shapiro (1970) smoothing operator is applied to perturbation values of surface pressure, temperature, and water vapor mixing ratio, and zonally on the wind field once per hour (cf. Liang 1986). A 9-point horizontal areal smoothing of the lapse rate and a three dimensional smoothing of the diabatic heating are also performed. See also Time Integration Scheme(s).

- Filling of spurious negative values of moisture is accomplished by application of the numerical scheme of Liang (1986) to the advection of atmospheric water vapor.

\section{Sampling Frequency}

For the AMIP simulation, the model history is written every 6 hours.

\section{Dynamical/Physical Properties}

\section{Atmospheric Dynamics}

Primitive-equations dynamics are expressed in terms of wind velocity, temperature, specific humidity, and a pressure parameter (PS-PT), where PS is the surface pressure and PT is $200 \mathrm{hPa}$, the pressure of the dynamical top of the model (see Vertical Representation). The dynamical framework utilizes perturbations from the temperature, geopotential, and surface pressure of the model's standard atmosphere (cf. Zeng 1979, Zeng et al. 1987, and Zeng et al. 1989).

\section{Diffusion}

- Nonlinear horizontal diffusion of heat, momentum, and moisture following Smagorinsky (1963) and Washington and Williamson (1977) is applied on the modified sigma surfaces (see Vertical Representation).

- There is no vertical diffusion as such, but momentum may be redistributed between the two atmospheric layers (see Vertical Resolution) by either eddy viscosity or convective friction. When the latter dominates, the friction coefficient depends 
on whether midlevel or penetrative convection occurs (see Convection). Cf. Zeng et al. (1989) for further details.

\section{Gravity-wave Drag}

Gravity-wave drag is not modeled.

\section{Solar Constant/Cycles}

The solar constant is the AMIP-prescribed value of $1365 \mathrm{~W} \mathrm{~m}^{-2}$. Both seasonal and diurnal cycles in solar forcing are simulated.

\section{Chemistry}

The carbon dioxide concentration is the AMIP-prescribed value of $345 \mathrm{ppm}$. Above $200 \mathrm{hPa}$ (the model dynamical top), a vertically integrated zonal ozone profile is specified from data of Duetsch (1971), and is updated daily by linear interpolation between the 15th day of consecutive months. The radiative effects of water vapor, but not of aerosols, are also included (see Radiation).

\section{Radiation}

- Shortwave radiation is calculated in ultraviolet (wavelengths $<0.5$ micron) and visible (wavelengths 0.5-0.9 micron) spectral intervals, employing a delta-Eddington approximation (cf. Cess 1985 and Cess et al. 1985). The shortwave calculations include treatment of Rayleigh scattering, absorption by water vapor using the exponential sum-fit method of Somerville et al. (1974), absorption by ozone following Cess and Potter (1987) and Lacis and Hansen (1974), and scattering/absorption by cloud droplets. The optical properties (single-scattering albedo, asymmetry factor, and optical depth) of these droplets depend on cloud type (see Cloud Formation).

- Longwave absorption by carbon dioxide and water vapor, with empirical transmission functions after Katayama (1972), is calculated for five spectral intervals with wavelengths $>0.9$ micron. Cirrus clouds or cirrus anvils on convective clouds (see Cloud Formation) are treated as graybodies (emissivity of 0.5 , expressed as a modified fractional cloudiness), and other clouds as blackbodies (emissivity of 1.0). For purposes of the radiation calculations, blackbody clouds overlap fully in the vertical, but graybody clouds only partially. Cf. Zeng et al. (1989) for further details. 


\section{Convection}

- The parameterization of convection after Arakawa et al. (1969) includes these elements: dry convective adjustment; shallow, midlevel, and penetrative convection; determination of the cumulus mass flux; and modification of the planetary boundary layer (PBL). If either atmospheric layer (see Vertical Resolution) is dry adiabatically unstable, the potential temperatures are adjusted to a common value, such that dry static energy is conserved. The layers that display evidence of moist convective instability determine whether (mutually exclusive) shallow, midlevel, or penetrative convection occur. Precipitation is associated with midlevel and penetrative convection (see Precipitation), and changes in PBL temperature and humidity are associated with shallow and penetrative convection (see Planetary Boundary Layer). Convective clouds are assumed to form a steady-state ensemble, with cloud air being saturated; the vertical profile of cloud temperature is computed assuming that moist static energy is conserved.

- Cumulus mass flux is computed (differently) for midlevel and penetrative convection, assuming that the convective instability is removed with an e-folding time of 1 hour. (Cumulus mass flux is not associated with shallow convection in the model.) The mass flux from the PBL and that entrained from the free atmosphere are mixed in the lower part of the convective cloud, with detrainment of this mixture at the level of nonbuoyancy. The magnitude of the mass flux determines the amount of cumulus friction (with different coefficients for midlevel and penetrative convection), which brings about momentum transfer between the two atmospheric layers (see Diffusion). Cf. Zeng et al. (1989) for further details.

\section{Cloud Formation}

- Clouds may result either from large-scale condensation or from convection (see Convection). Large-scale clouds form when the relative humidity exceeds 90 percent in the lower vertical layer or 100 percent in the upper layer (see Vertical Resolution). Clouds are assumed to fill a grid box (cloud fraction $=1$ ). (For purposes of longwave radiation calculations, however, graybody cirrus and cirrus anvil cloud fractions are 0.5.)

- Four basic cloud types are modeled. Type 1 is formed by either midlevel or penetrative convection, type 2 when the relative humidity of the lower vertical layer exceeds 90 percent, type 3 as a result of shallow convection, and type 4 when large-scale 
precipitation occurs in the upper vertical layer (see Precipitation). (Precipitation is limited to cloud types 1,2, and 4.) Types 2,3, or 4 are defined as graybody cirrus clouds if they are nonprecipitating or if the average of their base and top temperatures is $<-40^{\circ} \mathrm{C}$; convective cloud type 1 is also capped by a graybody cirrus anvil if it satisfies this temperature criterion.

- In addition, a cloud type 5 is formed by the coexistence of types 2 and 4 , and a cloud type 6 by the coexistence of types 3 and 4 . (Types 1 and 3 cannot coexist; type 1 also overrides the formation of types 2 and 4 , while type 2 overrides type 3.) Both types 5 and 6 are treated as low-level clouds for radiation purposes. Cf. Zeng et al. (1989) for further details. See also Radiation.

\section{Precipitation}

- Precipitation may result from midlevel or penetrative convection (see Convection). The amount of precipitation is equal to the net water vapor entrained from the environment, and falls at a rate that is a function of the cumulus mass flux. There is no subsequent evaporation of convective precipitation.

- Precipitation also results from large-scale condensation. When the upper atmospheric layer becomes supersaturated, the resulting precipitation is assumed to evaporate completely in passing through the lower layer, thereby cooling and moistening the environment in proportion to the amount of evaporation (cf. Lowe and Ficke 1974). If the lower layer then becomes supersaturated, the resulting precipitation falls to the surface.

\section{Planetary Boundary Layer}

The PBL is parameterized as a constant flux surface layer of indefinite thickness (see Surface Fluxes). Its temperature and humidity are modified by shallow and penetrative convection (see Convection), with new values computed assuming conservation of moist static energy in the vertical.

\section{Orography}

The model's orography is determined by area-averaging the $1 \times 1$-degree topographic data of Gates and Nelson (1975) within each $4 \times 5$-degree grid box. A 9-point smoothing of orography on neighboring model grid squares is also performed. 


\section{IAP}

\section{Ocean}

AMIP monthly sea surface temperatures are prescribed, with intermediate daily values determined by linear interpolation.

\section{Sea Ice}

AMIP monthly sea ice extents are prescribed. The surface temperature of the ice is determined from a budget equation that includes the surface heat fluxes (see Surface Fluxes) plus conduction heating from the ocean below the ice. This subsurface flux is a function of the heat conductivity and thickness (a constant $3 \mathrm{~m}$ ) of the ice, and of the difference between the predicted ice temperature and that prescribed $(271.5 \mathrm{~K})$ for the ocean below. Snow is allowed to accumulate on sea ice (see Snow Cover), and melts if the ice surface temperature is $>0{ }^{\circ} \mathrm{C}$.

\section{Snow Cover}

Precipitation falls as snow if the surface air temperature is $<0^{\circ} \mathrm{C}$. The snow mass is determined from a budget equation that includes the rates of snow accumulation, melting, and sublimation. Snowmelt (which contributes to soil moisture--see Land Surface Processes) is computed from the difference between the downward heat fluxes at the surface and the upward heat fluxes that would occur for a surface temperature equal to the melting temperature of ice $\left(0^{\circ} \mathrm{C}\right)$. (For snow on sea ice, the conduction heat flux from the ocean below also contributes to snowmelt--see Sea Ice.) The sublimation rate is set equal to the surface evaporative flux (see Surface Fluxes) unless all the snow mass is removed in less than one hour; in this case, sublimation is equated to the rate of snow mass removal. Cf. Zeng et al. (1989) for further details.

\section{Surface Characteristics}

- Roughness lengths are not specified, since these are not required for the calculation of turbulent fluxes (see Surface Fluxes).

- The surface albedo is prescribed for water, land ice, sea ice, and for six land surface types. Following Manabe and Holloway (1975), the albedo of snow-covered surfaces varies as the square root of snow mass up to a maximum value that is assigned for a critical snow mass of $10 \mathrm{~kg} \mathrm{~m}^{-2}$ (see Snow Cover). Albedos for the land surfaces (with and without snow cover) are assigned from data of Posey and Clapp (1964). Over 
water, the albedo for diffuse solar flux is taken as 0.07 , and that for the direct beam is a function of solar zenith angle (cf. Zeng et al. 1989).

- Longwave emissivity is prescribed as unity (blackbody emission) for all surface types.

\section{Surface Fluxes}

- The absorbed surface solar flux is determined from the surface albedo, and surface longwave emission from the Planck function with constant surface emissivity of 1.0 (see Surface Characteristics).

- Turbulent surface fluxes of momentum, heat, and moisture are calculated from bulk aerodynamic formulae. The momentum flux is proportional to the product of a drag coefficient and the effective surface wind, which is determined by extrapolating the wind at the two vertical levels and multiplying by a factor of 0.7 (but constrained to be at least $2 \mathrm{~m} \mathrm{~s}^{-1}$ in magnitude). The drag coefficient is not a function of vertical stability but it does depend on surface elevation. Over the oceans, the drag coefficient is a function of the surface wind speed, but it is constrained to be at most $2.5 \times 10^{-3}$.

- The surface heat and moisture fluxes also depend on a product of the same drag coefficient and effective surface wind speed, as well as on the difference between the ground and surface atmospheric temperatures (for heat fluxes) or specific humidities (for moisture fluxes). These surface values of atmospheric temperature and humidity are determined by equating, respectively, the surface sensible heat and evaporative flux to corresponding fluxes at the top of the constant-flux surface layer. The latter are parameterized following K-theory (cf. Arakawa 1972), where the eddy diffusivity depends on vertical stability but is constrained to be $<15 \mathrm{~m}^{2} \mathrm{~s}^{-1}$ over water surfaces, and to be $<100 \mathrm{~m}^{2} \mathrm{~s}^{-1}$ elsewhere. The surface moisture flux also depends on an evapotranspiration efficiency factor $\beta$, which is taken as unity over snow, ice, and water, and in areas of dew formation. Over land, $\beta$ is a function of soil moisture. Cf. Zeng et al. (1989) for further details.See also Diffusion, Planetary Boundary Layer, and Land Surface Processes.

\section{Land Surface Processes}

- Following Priestly (1959) and Bhumralkar (1975) the average ground temperature at the diurnal skin depth is computed from the net surface energy 


\section{IAP}

fluxes (see Surface Fluxes), taking account of the thermal conductivity, and the volumetric and bulk heat capacities of snow, ice, and soil.

- Soil moisture is predicted as a fraction of a uniform field capacity of $0.15 \mathrm{~m}$ in a single layer (i.e., a "bucket" model). Fractional soil moisture is determined from a budget that includes the rates of precipitation, snowmelt, surface evaporation, and runoff. The evapotranspiration efficiency $\beta$ over land (see Surface Fluxes) is specified as the lesser of twice the fractional soil moisture or unity. Runoff is given by the product of the fractional soil moisture and the sum of precipitation and snowmelt rates. If the predicted fractional soil moisture is in excess of unity, the excess is taken as additional runoff. Cf. Zeng et al. (1989) for further details. 


\section{JMA: Japan Meteorological Agency}

\section{AMIP Representative(s)}

Dr. Nobuo Sato, Dr. Toshiki Iwasaki, and Dr. Tadashi Tsuyuki, Numerical Prediction Division, Japan Meteorological Agency, 1-3-4 Ote-machi, Chiyoda-ku, Tokyo 100 Japan; Phone: + 81-03-3212-8341; Fax: +81-03-3211-8407;

e-mail: $/ \mathrm{PN}=\mathrm{N} . \mathrm{SATO} / \mathrm{O}=\mathrm{JMA} / \mathrm{ADMD}=\mathrm{ATI} / \mathrm{C}=\mathrm{JP} / @$ sprint.com (Sato)

$/ \mathrm{PN}=\mathrm{T} . \mathrm{IWASAKI} / \mathrm{O}=\mathrm{JMA} / \mathrm{ADMD}=\mathrm{ATI} / \mathrm{C}=\mathrm{JP} / \mathrm{Sprint}_{\mathrm{S}}$.com (Iwasaki)

$/ \mathrm{PN}=\mathrm{T} . \mathrm{TSUYUKL} / \mathrm{O}=\mathrm{JMA} / \mathrm{ADMD}=\mathrm{ATL} / \mathrm{C}=\mathrm{JP} / @$ sprint.com (Tsuyuki)

\section{Model Designation}

JMA GSM8911 T42L21

\section{Model Lineage}

The JMA GSM8911 model first became operational in November 1989. This version is derived from an earlier global spectral model that is described by Kanamitsu et al. (1983).

\section{Model Documentation}

Key documentation of the model is provided by the Numerical Prediction Division's 1993 Outline of Operational Numerical Weather Prediction at Japan Meteorological Agency (hereafter Numerical Prediction Division 1993) and by Sugi et al. (1989).

\section{Numerical/Computational Properties}

\section{Horizontal Representation}

Spectral (spherical harmonic basis function) with transformation to a Gaussian grid for calculation of nonlinear quantities and some physics.

Horizontal Resolution

Spectral triangular 42 (T42), roughly equivalent to a $2.8 \times 2.8$ degree latitudelongitude grid. 
JMA

\section{Vertical Domain}

Surface to $10 \mathrm{hPa}$. For a surface pressure of $1000 \mathrm{hPa}$, the lowest atmospheric level is at a pressure of $995 \mathrm{hPa}$.

\section{Vertical Representation}

Hybrid vertical coordinates which approximate conventional sigma coordinates at low levels and constant-pressure coordinates at upper levels (cf. Simmons and Burridge 1981).

\section{Vertical Resolution}

There are 21 unevenly spaced hybrid levels. For a surface pressure of $1000 \mathrm{hPa}$, 6 levels are below $800 \mathrm{hPa}$ and 7 levels are above $200 \mathrm{hPa}$.

\section{Computer/Operating System}

The AMIP simulation was run on a HITAC S-810 computer using a single processor in the HITAC VOS3/HAP/ES operational environment.

\section{Computational Performance}

For the AMIP experiment, about 2 minutes of HITAC S-810 computation time per simulated day.

\section{Initialization}

For the AMIP simulation, the initial model atmospheric state is specified from the ECMWF FGGE III-B analysis for 1 January 1993, with a nonlinear normalmode initialization also applied (cf. Kudoh 1984). Soil moisture is initialized according to estimates of Willmott et al. (1985), and snow cover/depth according to data of Dewey (1987).

\section{Time Integration Scheme(s)}

Semi-implicit leapfrog time integration with an Asselin (1972) time filter (cf. Jarraud et al. 1982). The length of the time step is not fixed, but is reset every 6 hours to satisfy the Courant-Friedrichs-Lewy (CFL) condition for the advection terms. Shortwave radiation is recalculated hourly, and longwave radiation every 3 hours. 


\section{Smoothing / Filling}

Orography is truncated at the T42 model resolution (see Orography). When the atmospheric moisture content of a grid box becomes negative due to spectral truncation, its value is reset to zero without any other modification of the local or global moisture budgets.

\section{Sampling Frequency}

For the AMIP simulation, the model history is written every 6 hours, but some diagnostic variables are stored only once per month because of limited storage resources.

\section{Dynamical/Physical Properties}

\section{Atmospheric Dynamics}

Primitive equation dynamics are expressed in terms of vorticity, divergence, temperature, specific humidity, and surface pressure, as formulated by Simmons and Burridge (1981) for hybrid vertical coordinates.

\section{Diffusion}

- Fourth order linear $\left(\nabla^{4}\right)$ horizontal diffusion is applied to vorticity, divergence, temperature, and specific humidity on the hybrid vertical surfaces, but with a firstorder correction of the temperature and moisture equations to approximate diffusion on constant-pressure surfaces (thereby reducing spurious mixing along steep mountain slopes). Diffusion coefficients are chosen so that the enstrophy power spectrum coincides with that expected from two-dimensional turbulence theory.

- Stability-dependent vertical diffusion of momentum, heat, and moisture in the planetary boundary layer (PBL) as well as in the free atmosphere follows the Mellor and Yamada (1974) level-2 turbulence closure scheme. The eddy diffusion coefficient is diagnostically determined from a mixing length formulated after the method of Blackadar (1962). See also Planetary Boundary Layer and Surface Fluxes. 


\section{Gravity-wave Drag}

Orographic gravity-wave drag is parameterized by two schemes that differ mainly in the vertical partitioning of the momentum deposit, depending on the wavelength of the gravity waves. Long waves (wavelengths $>100 \mathrm{~km}$ ) are assumed to exert drag mainly in the stratosphere (type A scheme), and short waves (wavelengths $\approx 10 \mathrm{~km}$ ) to deposit momentum only in the troposphere (type B scheme). In both schemes the gravity-wave drag stress is a function of atmospheric density, wind, the Brunt-Vaisalla frequency, and subgrid-scale orographic variance (see Orography). (For the type B scheme, orographic variance is computed as an average difference of maximum and minimum heights within each 10-minute mesh.) In the type A scheme, the deposition of vertical momentum is determined from a modified Palmer et al. (1986) amplitude saturation hypothesis. Because the momentum stress of short gravity waves decreases with altitude as a result of nonhydrostatic effects (cf. Wurtele et al. 1987), the type B scheme assumes the wave stress to be quadratic in pressure and to vanish around the tropopause. Cf. Iwasaki et al. (1989a, b) for further details.

\section{Solar Constant/Cycles}

The solar constant is the AMIP-prescribed value of $1365 \mathrm{~W} \mathrm{~m}^{-2}$. Both seasonal and diurnal cycles in solar forcing are simulated.

\section{Chemistry}

The carbon dioxide concentration is the AMIP-prescribed value of $345 \mathrm{ppm}$. Monthly averaged zonal ozone distributions are specified from data of McPeters et al. (1984). Radiative effects of water vapor, but not of aerosols, are also included (see Radiation).

\section{Radiation}

- Shortwave radiation is parameterized differently for wavelengths $<0.9$ micron (visible) and > 0.9 micron (near-infrared). In the visible, absorption by ozone, Rayleigh scattering by air molecules, and Mie scattering by cloud droplets are treated. In the nearinfrared, water vapor absorption is modeled after Lacis and Hansen (1974). Nearinfrared scattering and absorption by cloud droplets are calculated by the deltaEddington approximation with constant single-scattering albedo.

- Longwave absorption by water vapor, ozone, and carbon dioxide is determined from transmission functions of Rodgers and Walshaw (1966), Goldman and Kyle (1968), 
and Houghton (1977), respectively; pressure broadening effects are also included. Continuum absorption by water vapor is treated by the method of Roberts et al. (1976). Transmission in four spectral bands (with boundaries at $4.0 \times 10^{3}, 5.5 \times 10^{4}, 8.0 \times 10^{4}$, $1.2 \times 10^{5}$, and $2.2 \times 10^{5} \mathrm{~m}^{-1}$ ) includes overlapping effects of different absorbers. Longwave emissivity of cirrus cloud is set at 0.80 , and that of all other clouds at 1.0 (blackbody emission). For purposes of the radiation calculations, all clouds are assumed to be randomly overlapped in the vertical. Cf. Sugi et al. (1989) for further details. See also Cloud Formation.

\section{Convection}

- A modified Kuo (1974) parameterization is used to simulate deep convection. The criteria for the occurrence of convection include conditionally unstable stratification and positive moisture convergence between the cloud base and top. The cloud base is at the lifting condensation level for surface air, and the top is at a level where the cloud and environmental temperatures are identical. The cloud temperature is determined from the moist adiabatic lapse rate modified by height-dependent entrainment, as proposed by Simpson and Wiggard (1969). In a vertical column, the total moisture available from convergence is divided between a fraction $b$ that moistens the environment and the remainder $(1-b)$ that contributes to the latent heating (rainfall) rate. The moistening parameter $b$ is a cubic function of the ratio of the mean relative humidity of the cloud layer to a prescribed critical relative humidity threshold value (70 percent); if cloud relative humidity is less than this threshold, $b$ is set to unity (no heating of the environment).

- Shallow convection occurs where the vertical stratification is conditionally unstable but moisture convergence is negative. It is parameterized by enhancing the vertical diffusion coefficients after the method of Tiedtke (1983).

\section{Cloud Formation}

No explicit convective cloud fraction is determined (see Convection). The stratiform cloud fraction is a quadratic function of the difference between the local relative humidity and a critical value that is empirically obtained from satellite observations, and that varies for low, middle, and high clouds (cf. Saito and Baba 1988). See also Radiation for treatment of cloud-radiative interactions. 


\section{Precipitation}

The convective precipitation rate is determined from the variable moistening parameter b in the modified Kuo (1974) convection scheme (see Convection). Any remaining supersaturation is removed by large-scale condensation. No subsequent evaporation of precipitation is simulated. See also Snow Cover.

\section{Planetary Boundary Layer}

The Mellor and Yamada (1974) level-2 turbulence closure scheme (see Diffusion) represents the effects of the PBL through the determination of the Richardson number and the vertical wind shear. The PBL top is not explicitly computed. See also Surface Fluxes.

\section{Orography}

Orography is obtained from a U.S. Navy dataset (cf. Joseph 1980) with resolution of 10 minutes arc on a latitude-longitude grid. These data are expressed as a series of spherical harmonics that are truncated at the T42 model resolution. Orographic variances that are also obtained from this dataset are used in the parameterization of gravity-wave drag (see Gravity-wave Drag).

\section{Ocean}

AMIP monthly sea surface temperature fields are prescribed, with daily values determined by linear interpolation.

Sea Ice

Monthly AMIP sea ice extents are prescribed. The ice surface temperature is predicted by the force-restore method of Deardorff (1978). The forcing includes the net balance of surface energy fluxes (see Surface Fluxes) as well as conduction heating from the ocean below, which is computed assuming the ice to be a uniform $2 \mathrm{~m}$ thick and the ocean to be at the temperature for sea ice formation (about $-2^{\circ} \mathrm{C}$ ). Snow is not allowed to accumulate on sea ice (see Snow Cover).

\section{Snow Cover}

Precipitation falls as snow if the temperature at the lowest atmospheric level (see Vertical Domain) is $<0^{\circ} \mathrm{C}$. Snow may accumulate on land, but not on sea ice. The 
fractional coverage of a grid box is proportional to the water-equivalent snow depth up to $0.02 \mathrm{~m}$; at greater depths, the proportionality constant varies with vegetation type. Snow cover alters the roughness and the albedo of bare and vegetated ground as well as the heat capacity and conductivity of soil, but sublimation from snow is not included in the surface evaporative flux. Snow melts (and contributes to soil moisture) if the ground surface temperature is $>0{ }^{\circ} \mathrm{C}$. See also Surface Characteristics, Surface Fluxes, and Land Surface Processes.

\section{Surface Characteristics}

- Over land, the 12 vegetation/surface types of the Simple Biosphere ( $\mathrm{SiB}$ ) model of Sellers et al. (1986) are specified at monthly intervals.

- The local roughness length over land varies monthly according to vegetation type (cf. Dorman and Sellers 1989); it decreases with increasing snow depth, the minimum value being 5 percent of that without snow cover. The surface roughness of sea ice is a uniform $1 \times 10^{-3} \mathrm{~m}$. Over oceans, the roughness length for momentum is a function of the surface wind stress after Charnock (1955), while the roughness length for surface heat and moisture fluxes is specified as a constant $1.52 \times 10^{-4} \mathrm{~m}$ (cf. Kondo 1975).

- Over land, surface albedos vary monthly according to seasonal changes in vegetation (cf. Dorman and Sellers 1989). The albedo is specified separately for visible (0.0-0.7 micron) and near-infrared (0.7-4.0 microns) spectral intervals, and is also a function of solar zenith angle. Following Sellers et al. (1986), snow cover alters the surface albedo. Over oceans and sea ice, albedos are functions of solar zenith angle but are independent of spectral interval.

- Longwave emissivity is prescribed to be unity (blackbody emission) for all surfaces. See also Surface Fluxes and Land Surface Processes.

\section{Surface Fluxes}

- Solar absorption at the surface is determined from the albedo, and longwave emission from the Planck equation with prescribed emissivity of 1.0 (see Surface Characteristics).

- The representation of turbulent surface fluxes of momentum, heat, and moisture follows Monin-Obukhov similarity theory as expressed by bulk formulae. The wind, temperature, and humidity required for these formulae are taken to be the 
values at the lowest atmospheric level (at $995 \mathrm{hPa}$ for a surface pressure of $1000 \mathrm{hPa}$ ). The associated drag/transfer coefficients are functions of the surface roughness (see Surface Characteristics) and vertical stability, following Louis et al. (1981).

- Over vegetated surfaces, the temperature and specific humidity of the vegetation canopy space of the SiB model of Sellers et al. (1986) are used as surface atmospheric values. Over land, the surface moisture flux includes evapotranspiration from dry vegetation (reflecting the presence of stomatal and canopy resistances) as well as direct evaporation from the wet canopy and from bare soil (see Land Surface Processes).

\section{Land Surface Processes}

- Land surface processes are simulated by the SiB model of Sellers et al. (1986), as implemented by Sato et al. (1989a, b). Vegetation in each grid box may consist both of ground cover and an upper-story canopy, with the spatial pattern of the ground cover varying monthly. Within the canopy, evaporative fluxes are computed by the PenmanMonteith method (cf. Monteith 1973). Evapotranspiration from dry leaves includes the detailed modeling of stomatal and canopy resistances. Direct evaporation from the wet canopy and from bare soil is also treated (see Surface Fluxes). Precipitation interception by the canopy (with large-scale and convective precipitation distinguished) is simulated, and infiltration of moisture into the ground is limited to less than the local hydraulic conductivity of the soil.

- Soil temperature is predicted in four layers by the force-restore method of Deardorff (1978). Soil liquid moisture is predicted from budget equations in three layers, and snow and soil ice in four layers. This moisture is increased by infiltrated precipitation and snowmelt, and is depleted by evapotranspiration and direct evaporation. Both surface runoff and deep runoff from gravitational drainage are simulated. See also Surface Characteristics and Surface Fluxes. 


\section{LMD: Laboratoire de Météorologie Dynamique}

\section{AMIP Representative(s)}

Dr. Herve Le Treut, Laboratoire de Météorologie Dynamique du Centre National de la Recherche Scientifique, Ecole Normale Superieure, 24 Rue Lhomond, 75231 Paris Cedex 05, France; Phone: +33-1-43-29-12-25; Fax: +33-1-43-36-83-92; e-mail: letreut@lmd.ens.fr.

\section{Model Designation}

LMD LMD5 3.6x5.6L11

\section{Model Lineage}

The LMD model derives from an earlier version developed for climate studies (cf. Sadourny and Laval 1984). Subsequent modifications principally include changes in the representation of radiation and horizontal diffusion, and inclusion of parameterizations of gravity-wave drag and prognostic cloud formation.

\section{Model Documentation}

Key documentation of the LMD model is provided by Sadourny and Laval (1984), Laval et al. (1981), and Le Treut and Li (1991). Details of computational aspects are described by Butel (1991).

\section{Numerical/Computational Properties}

\section{Horizontal Representation}

Finite differences on a uniform-area, staggered C-grid (cf. Arakawa and Lamb 1977), with points equally spaced in sine of latitude and in longitude. See also Horizontal Resolution.

\section{Horizontal Resolution}

There are 50 grid points equally spaced in the sine of latitude and 64 points equally spaced in longitude. (The mesh size is $225 \mathrm{~km}$ north-south and $625 \mathrm{~km}$ eastwest at the equator, and is about $400 \times 400 \mathrm{~km}$ at 50 degrees latitude. ) 


\section{Vertical Domain}

Surface to about $4 \mathrm{hPa}$. For a surface pressure of $1000 \mathrm{hPa}$, the lowest atmospheric level is at $979 \mathrm{hPa}$.

\section{Vertical Representation}

Finite-difference sigma coordinates.

\section{Vertical Resolution}

There are 11 unevenly spaced sigma levels. For a surface pressure of $1000 \mathrm{hPa}$, 3 levels are below $800 \mathrm{hPa}$ and 2 levels are above $200 \mathrm{hPa}$.

\section{Computer/Operating System}

The AMIP simulation was run on a Cray 2 computer, using a single processor in the UNICOS operating environment.

\section{Computational Performance}

For the AMIP experiment, about 2 minutes Cray 2 computation time per simulated day.

\section{Initialization}

For the AMIP experiment, the model atmosphere, soil moisture, and snow cover/ depth are initialized for 1 January 1979 from a previous model simulation.

\section{Time Integration Scheme(s)}

The time integration scheme for dynamics combines 4 leapfrog steps with a Matsuno step, each of length 6 minutes. Model physics is updated every 30 minutes, except for shortwave/longwave radiative fluxes, which are calculated every 6 hours. For computation of vertical turbulent surface fluxes and diffusion, an implicit backward integration scheme with 30-minute time step is used, but with all coefficients calculated explicitly. See also Surface Fluxes and Diffusion.

\section{Smoothing / Filling}

Orography is area-averaged on the model grid (see Orography). At the four latitude points closest to the poles, a Fourier filtering operator after Arakawa and Mintz (1974) is 
applied to the momentum, thermodynamics, continuity, and water vapor tendency equations to slow the longitudinally propagating gravity waves for numerical stability. Negative moisture values (arising from vertical advection by the centered nondiffusive scheme) are filled by borrowing moisture from the level below.

\section{Sampling Frequency}

For the AMIP simulation, the model history is written once every 24 hours.

\section{Dynamical/Physical Properties}

\section{Atmospheric Dynamics}

Primitive-equation dynamics are expressed in terms of $\mathrm{u}$ and $\mathrm{v}$ winds, potential enthalpy, specific humidity, and surface pressure. The advection scheme is designed to conserve potential enstrophy for divergent barotropic flow (cf. Sadourny 1975a, b). Total energy is also conserved for irrotational flow (cf. Sadourny 1980). The continuity and thermodynamics equations are expressed in flux form, conserving mass and the space integrals of potential temperature and its square. The water vapor tendency is also expressed in flux form, thereby reducing the probability of spurious negative moisture values (see Smoothing / Filling).

\section{Diffusion}

- Linear horizontal diffusion is applied on constant-pressure surfaces to potential enthalpy, divergence, and rotational wind via a biharmonic operator $\nabla\left(\nabla^{*} \nabla^{*}\right) \nabla$, where $\nabla$ denotes a first-order difference on the model grid, while $\nabla^{*}$ is a formal differential operator on a regular grid without geometrical corrections. Because of the highly diffusive character of the flux-form water vapor tendency equation (see Atmospheric Dynamics), no further horizontal diffusion of specific humidity is included. Cf. Michaud (1987) for further details.

- Second-order vertical diffusion of momentum, heat, and moisture is applied only within the planetary boundary layer (PBL). The diffusion coefficient depends on a diagnostic estimate of the turbulence kinetic energy (TKE) and on the mixing length (which decreases up to the prescribed PBL top) that is estimated after Smagorinsky et al. (1965). Estimation of TKE involves calculation of a countergradient term after Deardorff (1966) and 
comparison of the bulk Richardson number with a critical value. Cf. Sadourny and Laval (1984) for further details. See also Planetary Boundary Layer and Surface Fluxes.

\section{Gravity-wave Drag}

The formulation of gravity-wave drag closely follows the linear model described by Boer et al. (1984a). The drag at any level is proportional to the vertical divergence of the wave momentum stress, which is formulated as the product of a constant aspect ratio, the local Brunt-Vaisalla frequency, a launching height determined from the orographic variance over the grid box (see Orography), the local wind velocity, and its projection on the wind vector at the lowest model level. The layer where gravity-wave breakdown occurs (due to convective instability) is determined from the local Froude number; in this critical layer the wave stress decreases quadratically to zero as a function of height.

\section{Solar Constant/Cycles}

The solar constant is the AMIP-prescribed value of $1365 \mathrm{~W} \mathrm{~m}^{-2}$. A seasonal, but not a diurnal cycle in solar forcing, is simulated.

\section{Chemistry}

The carbon dioxide concentration is the AMIP-prescribed value of $345 \mathrm{ppm}$. Three-dimensional ozone concentration is diagnosed as a function of the $500 \mathrm{hPa}$ geopotential heights following the method of Royer et al. (1988). Radiative effects of water vapor, but not those of aerosols, are also included (see Radiation).

\section{Radiation}

- Shortwave radiation is modeled after an updated scheme of Fouquart and Bonnel (1980). Upward/downward shortwave irradiance profiles are evaluated in two stages. First, a mean photon optical path is calculated for a scattering atmosphere including clouds and gases. The reflectance and transmittance of these elements are calculated by, respectively, the delta-Eddington method (cf. Joseph et al. 1976) and by a simplified two-stream approximation. The scheme evaluates upward/downward shortwave fluxes for two reference cases: a conservative atmosphere and a first-guess absorbing atmosphere; the mean optical path is then computed for each absorbing gas 
from the logarithm of the ratio of these reference fluxes. In the second stage, final upward/downward fluxes are computed for two spectral intervals (0.30-0.68 micron and 0.68-4.0 microns) using more exact gas transmittances (Rothman 1981) and with adjustments made for the presence of clouds (see Cloud Formation). For clouds, the asymmetry factor is prescribed, and the optical depth and single-scattering albedo are functions of cloud liquid water content after Stephens (1978b).

- Longwave radiation is modeled in six spectral intervals between wavenumbers 0 and $2.82 \times 10^{5} \mathrm{~m}^{-1}$ after the method of Morcrette $(1990,1991)$. Absorption by water vapor (in two intervals), by the water vapor continuum (in two intervals in the atmospheric window, following Clough et al. 1980), by the carbon dioxide and the rotational part of the water vapor spectrum (in one interval), and by ozone (in one interval) is treated. The temperature and pressure dependence of longwave absorption by gases is included. Clouds are treated as graybodies in the longwave, with emissivity depending on cloud liquid water path after Stephens (1978b). Longwave scattering by cloud droplets is neglected, and droplet absorption is modeled by an emissivity formulation from the cloud liquid water path. For purposes of the radiation calculations, all clouds are assumed to overlap randomly in the vertical. See also Cloud Formation.

\section{Convection}

- When the temperature lapse rate is conditionally unstable, subgrid-scale convective condensation takes place. If the air is supersaturated, a moist convective adjustment after Manabe and Strickler (1964) is carried out: the temperature profile is adjusted to the previous estimate of the moist adiabatic lapse rate, with total moist static energy in the column being held constant. The specific humidity is then set to a saturated profile for the adjusted temperature lapse, and the excess moisture is rained out (see Precipitation).

- If the temperature lapse rate is conditionally unstable but the air is unsaturated, condensatiori also occurs following the Kuo (1965) cumulus convection scheme, provided there is large-scale moisture convergence. In this case, the lifting condensation level is assumed to be at the top of the PBL, and the height of the cumulus cloud is given by the highest level for which the moist static energy is less than that at the PBL top (see Planetary Boundary Layer). It is assumed that all the humidity entering each cloudy layer since the last call of the convective scheme (30 minutes prior) is 


\section{LMD}

pumped into this cloud. The environmental humidity is reduced accordingly, while the environmental temperature is taken as the grid-scale value; the cloud temperature and humidity profiles are defined to be those of a moist adiabat.

- The fractional area of the convective cloud is obtained from a suitably normalized, mass-weighted vertical integral (from cloud bottom to top) of differences between the humidities and temperatures of the cloud vs those of the environment. As a result of mixing, the environmental (grid-scale) temperature and humidity profiles evolve to the moist adiabatic values in proportion to this fractional cloud area, while the excess of moisture precipitates (see Precipitation). Mixing of momentum also occurs.

- There is no explicit simulation of shallow convection, but the moist convective adjustment produces similar effects in the moisture field (cf. Le Treut and Li 1991). See also Cloud Formation.

\section{Cloud Formation}

- Cloud cover is prognostically determined, as described by Le Treut and Li (1991). Time-dependent cloud liquid water content (LWC) follows a conservation equation involving rates of water vapor condensation, evaporation of cloud droplets, and the transformation of small droplets to large precipitating drops (see Precipitation). The LWC also determines cloud cover (see below) and cloud optical properties (see Radiation).

- The fraction of convective cloud in a grid box is unity if moist convective adjustment is invoked; otherwise, it is given by the surface fraction of the active cumulus cloud obtained from the Kuo (1965) scheme (see Convection). Cloud forms in those layers where there is a decrease in water vapor from one call of the convective scheme to the next (every 30 minutes), and the cloud LWC is redistributed in these layers proportional to this decrease.

- The fraction of straiiform cloud in any layer is determined from the probability that the total cloud water (liquid plus vapor) is above the saturated value. (A uniform probability distribution is assumed with a prescribed standard deviation--cloud typically begins to form when the relative humidity exceeds 83 percent of saturation.) This stochastic approach also crudely simulates the effects of evaporation of cloud droplets. Cf. Le Treut and Li (1991) for further details. See also Precipitation. 


\section{Precipitation}

Both convective and large-scale precipitation are linked to cloud LWC (see Cloud Formation). If the LWC exceeds a threshold value, all liquid water is assumed to precipitate. (For water clouds, the LWC threshold is set to $1 \times 10^{-3} \mathrm{~kg}$ liquid per $\mathrm{kg}$ dry air; for ice clouds with tops at temperatures below $-10^{\circ} \mathrm{C}$, the threshold is set to the maximum of 5 percent of the water vapor mixing ratio or $1 \times 10^{-2} \mathrm{~kg}$ per kg.) Evaporation of falling convective and large-scale precipitation is not explicitly modeled, but evaporation of small stratiform cloud droplets making up the LWC is simulated stochastically.

\section{Planetary Boundary Layer}

The PBL is represented by the first 4 levels above the surface (at sigma $=0.979$, $0.941,0.873$, and 0.770 ). The PBL top is prescribed to be at the sigma $=0.770$ level; here vertical turbulent eddy fluxes of momentum, heat, and moisture are assumed to vanish. See also Diffusion, Surface Fluxes, and Surface Characteristics.

\section{Orography}

Raw orography obtained at $10 \times 10$-minute resolution from the U.S. Navy dataset (cf. Joseph 1980) is area-averaged over the model grid boxes. The orographic variance about the mean value for each grid box is also computed from the same dataset for use in the gravity-wave drag parameterization (see Gravity-wave Drag).

\section{Ocean}

AMIP monthly sea surface temperature fields are prescribed, with daily values determined by a cubic-spline interpolation which preserves the mean.

Sea Ice

AMIP monthly sea ice extents are prescribed. The surface temperature of the ice is predicted from the balance of energy fluxes (see Surface Fluxes) that includes conduction heating from the ocean below. This conduction flux is proportional to the difference between the surface temperature and that of melting ice $(271.2 \mathrm{~K})$, and is inversely proportional to the ice thickness (prescribed to be a uniform $3 \mathrm{~m}$ ). Snow that accumulates on sea ice modifies its albedo and thermal properties. See also Snow Cover and Surface Characteristics. 


\section{LMD}

\section{Snow Cover}

If the air temperature at the first level above the surface (at sigma $=0.979$ ) is $<0{ }^{\circ} \mathrm{C}$, precipitation falls as snow. Prognostic snow mass is determined from a budget equation, with accumulation and melting over both land and sea ice. Snow cover affects the surface albedo and the heat capacity of the surface. Sublimation of snow is calculated as part of the surface evaporative flux, and snowmelt contributes to soil moisture. See also Surface Characteristics, Surface Fluxes, and Land Surface Processes.

\section{Surface Characteristics}

- The surface roughness lengths over the continents are prescribed as a function of orography and vegetation from data of Baumgartner et al. (1977), and their seasonal modulation is inferred following Dorman and Sellers (1989). Roughness lengths over ice surfaces are a uniform $1 \times 10^{-2} \mathrm{~m}$. Over ocean, the surface drag/transfer coefficients (see Surface Fluxes) are determined without reference to a roughness length.

- Surface albedos for oceans and snow-free sea ice are prescribed from monthly data of Bartman (1980), and for snow-free continents from monthly data of Dorman and Sellers (1989). When there is snow cover, the surface albedo is modified according to the parameterization of Chalita and Le Treut (1994), which takes account of snow age, eight types of vegetation background, and spectral range (in visible and nearinfrared subintervals).

- The longwave emissivity is prescribed as unity (blackbody emission) for all surfaces.

\section{Surface Fluxes}

- The surface solar absorption is determined from surface albedos, and longwave emission from the Planck equation with prescribed emissivity of 1.0 (see Surface Characteristics).

- In the lowest atmospheric layer, surface turbulent eddy fluxes of momentum, heat, and moisture are expressed as bulk formulae multiplied by drag/transfer coefficients that are functions of wind speed, stability, and (except over ocean) roughness length (see Surface Characteristics). The transfer coefficient for the surface moisture flux also depends on the vertical humidity gradient. Over ocean, the drag/transfer coefficients are determined (without reference to a roughness length) as functions of 
surface wind speed and temperature difference between the ocean and the surface air, following Bunker (1976).

- In addition, the momentum flux is proportional to the wind vector extrapolated to the surface. The sensible heat flux is proportional to the difference between the potential temperature at the ground and that extrapolated from the atmosphere to the surface. The surface moisture flux is proportional to the potential evaporation (the difference between the saturated specific humidity at the surface and the extrapolated atmospheric humidity) multiplied by an evapotranspiration efficiency $\beta$. Over oceans, snow, and ice, $\beta$ is set to unity, while over land it is a function of soil moisture (see Land Surface Processes).

- Above the surface layer, but only within the PBL, turbulent eddy fluxes are represented as diffusive processes (see Diffusion and Planetary Boundary Layer).

\section{Land Surface Processes}

- Ground temperature and bulk heat capacity (with differentiation for bare soil, snow, and ice) are defined as mean quantities over a single layer of thickness about $0.15 \mathrm{~m}$ (over which there is significant diurnal variation of temperature). The temperature prediction equation, which follows Corby et al. (1976), includes as forcing the surface heat fluxes (see Surface Fluxes) and the heat of fusion of snow and ice.

- Prognostic soil moisture is represented by a single-layer "bucket" model after Budyko (1956), with uniform field capacity $0.15 \mathrm{~m}$. Soil moisture is increased by both precipitation and snowmelt, and is decreased by surface evaporation, which is determined from the product of the evapotranspiration efficiency $\beta$ and the potential evaporation from a surface saturated at the local surface temperature and pressure (see Surface Fluxes). Over land, $\beta$ is given by the maximum of unity or twice the ratio of local soil moisture to the constant field capacity. Runoff occurs implicitly if the soil moisture exceeds the field capacity. Cf. Laval et al. (1981) for further details. 


\section{MGO: Main Geophysical Observatory}

\section{AMIP Representative(s)}

Dr. Valentin Meleshko, Voeikov Main Geophysical Observatory, 7 Karbyshev Str., 194018 St. Petersburg, Russia; Phone: +7-812-247-01-03; Fax: +7-812-247-86-61; e-mail: vmeleshk@sovam.com

\section{Model Designation \\ MGO AMIP92 T30L14}

\section{Model Lineage}

The MGO model was first employed in research in 1983 (cf. Meleshko et al. 1980 and Sokolov 1986). The current third generation model includes enhancements in the simulation of radiative transfer; vertical turbulent exchange of heat, moisture, and momentum between the surface and the atmosphere; and heat and moisture exchange in the soil.

\section{Model Documentation}

Key documentation of the model is provided by Meleshko et al. (1991).

\section{Numerical/Computational Properties}

\section{Horizontal Representation}

Spectral (spherical harmonic basis functions) with transformation to a Gaussian grid for calculation of nonlinear quantities and some physics.

\section{Horizontal Resolution}

The spectral triangular truncation is at total wave number 30 (T30), roughly equivalent to a $3.75 \times 3.75$-degree 1atitude-longitude grid.

\section{Vertical Domain}

Surface to $12.5 \mathrm{hPa}$. For a surface pressure of $1000 \mathrm{hPa}$, the lowest atmospheric level is at $992 \mathrm{hPa}$. 


\section{Vertical Representation}

Thermodynamically consistent finite difference formulation in sigma coordinates with momentum conservation (cf. Sheinin 1987 and Magazenkov and Sheinin 1988).

\section{Vertical Resolution}

There are 14 irregularly spaced sigma levels. For a surface pressure of $1000 \mathrm{hPa}$, 5 levels are below $800 \mathrm{hPa}$ and 4 levels are above $200 \mathrm{hPa}$.

\section{Computer/Operating System}

The AMIP simulation was run on a Cray 2 computer using a single processor in a UNICOS environment.

\section{Computational Performance}

For the AMIP experiment, about 6 minutes of Cray 2 computation time per simulated day.

\section{Initialization}

For the AMIP simulation, the model atmosphere is initialized for 1 January 1979 from ECMWF analyses. Initial conditions for soil moisture and snow cover/mass are taken from mean-January ECMWF climatologies.

\section{Time Integration Scheme(s)}

The main time integration is done by a two-step semi-implicit method (cf. Sheinin 1983), but an Euler-backward scheme is used for the vertical transport of momentum, heat, and moisture, which are split from other physical processes (see Surface Fluxes). The time step length is 30 minutes for dynamics and physics, except for full radiation calculations which are done once every 12 hours.

\section{Smoothing/Filling}

High-resolution topography is adjusted to the model's resolution by means of a special filter (see Orography). Negative values of moisture are filled by a horizontal and vertical borrowing procedure. 
MGO

Sampling Frequency

For the AMIP simulation, the model history is written once every 24 hours.

\section{Dynamical/Physical Properties}

\section{Atmospheric Dynamics}

Primitive-equation dynamics are expressed in terms of vorticity, divergence, virtual temperature, specific humidity, and surface pressure.

\section{Diffusion}

- There is second-order horizontal diffusion of vorticity, divergence, temperature, and specific humidity on sigma surfaces for total spectral wave numbers $>23$ (cf. Laursen and Eliasen 1989).

- Stability-dependent vertical diffusion of atmospheric momentum, temperature, and specific humidity is modeled (cf. Louis 1979).

\section{Gravity-wave Drag}

The formulation of gravity-wave drag follows McFarlane (1987). Deceleration of the resolved flow by dissipation of orographically excited gravity waves is a function of the rate at which the parameterized vertical component of the gravity-wave momentum flux decreases in magnitude with height. This momentum-flux term is the product of local air density, the component of the local wind in the direction of that at the near-surface reference level, and a displacement amplitude. At the surface, this amplitude is specified in terms of the subgrid-scale orographic variance, and in the free atmosphere by linear theory, but it is bounded everywhere by wave saturation values. See also Orography.

\section{Solar Constant/Cycles}

The solar constant is the AMIP-prescribed value of $1365 \mathrm{~W} \mathrm{~m}^{-2}$. A seasonal, but not a diurnal cycle in solar forcing, is simulated.

\section{Chemistry}

The carbon dioxide concentration is the AMIP-prescribed value of $345 \mathrm{ppm}$. The ozone concentration is prescribed as a function of latitude and season from Nimbus 7 
data (cf. McPeters et al. 1984). Radiative effects of water vapor and trace gases (methane, nitrous oxide, and chlorofluorocarbon compounds CFC-11 and CFC-12) are also included, but not those of aerosols (see Radiation). Global vertical profiles for the trace gases are specified from the U.S. Standard Model Atmosphere Profiles.

\section{Radiation}

- The shortwave fluxes are computed in three subranges: ultraviolet (0.2 to 0.31 micron), visible ( 0.31 to 0.75 micron), and near-infrared ( 0.75 to 4.0 microns). Fluxes in the ultraviolet and visible subranges are computed following Karol (1986). In the ultraviolet subrange, only ozone absorption is considered, and the effects of scattering are neglected. Within the visible subrange, absorption by ozone and Rayleigh scattering are accounted for using the delta-Eddington approximation. In the near-infrared sub-range, absorption by water vapor in 12 spectral bands and by carbon dioxide in 6 bands is determined using a Goody statistical band model (cf. Rozanov and Frolkis 1988).

- The longwave fluxes are computed in 9 spectral intervals over the range 0 to

$2.2 \times 10^{5} \mathrm{~m}^{-1}$, accounting for absorption by water vapor, carbon dioxide, ozone, and trace gases (see Chemistry). The flux computation is based on the Goody statistical band model, and its parameters depend on temperature (cf. Karol 1986). Water vapor continuum absorption is by the method of Roberts et al. (1976).

- The optical properties of clouds are prescribed (cf. Gordon et al. 1984), with cloud albedo depending on solar zenith angle. Convective cloud is assigned the same optical characteristics as the lower stratiform cloud. The longwave emissivity of high stratiform cloud depends on cloud temperature; it ranges linearly between 0.5 and 1.0 for temperatures from $-30^{\circ} \mathrm{C}$ to $-20^{\circ} \mathrm{C}$, and is fixed for temperatures beyond this range. All other clouds emit as blackbodies (emissivity of 1.0). For purposes of the radiation calculations, stratiform clouds of different types are assumed to be randomly overlapped in the vertical, and convective clouds to be fully overlapped. See also Cloud Formation.

\section{Convection}

-A Kuo (1974) scheme is used for simulation of deep convection (cf. also Louis 1984). Convection is assumed to occur only in the presence of conditionally unstable layers in the vertical and large-scale net moisture convergence in the horizontal. The associated 
convective cloud formed at the lifting condensation level is assumed to dissolve instantaneously through lateral mixing, thereby imparting heat and moisture to the environment. In a vertical column, the total moisture available from convergence is divided between a fraction $b$ that moistens the environment and the remainder $(1-b)$ that contributes to the latent heating (rainfall) rate. The value of $(1-b)$ is taken to be the ratio of the integral moisture content in a convective layer to the integral saturation moisture content of this layer (cf. Meleshko et al. 1991).

- Shallow convection is formulated as a convective adjustment process for moisture only.

\section{Cloud Formation}

- Cloud formation follows the diagnostic technique of Slingo (1987). There are 3 types of layer clouds (low, middle, and high) that are associated with large-scale disturbances. These clouds are present only when the relative humidity exceeds threshold values of 90,93 , and 98 percent for high, middle, and low layers, respectively. The layer cloud amount is a quadratic function of this humidity excess.

- The vertical depth of convective cloud is determined from the thickness of the unstable layers, and the areal extent from the logarithm of the convective precipitation rate (cf. Slingo 1987). Of the total cloud amount, 25 percent is assigned to the convective column clouds in the unstable layers, while 75 percent is assigned to the lowest unstable layer. Convective anvil cloud forms when the instability extends above the $400 \mathrm{hPa}$ level and the convective cloud fraction exceeds 0.20 ; the anvil cloud fraction cannot exceed 0.80. See also Radiation for treatment of cloudradiative interactions.

\section{Precipitation}

Large-scale precipitation forms when the local relative humidity exceeds 100 percent. Convective precipitation is determined from the complement of the moistening parameter $\mathrm{b}$ in the Kuo scheme (see Convection). Subsequent evaporation of precipitation is not simulated. See also Snow Cover.

\section{Planetary Boundary Layer}

The top of the boundary layer is assumed to coincide with the middle of the lowest atmospheric layer (sigma $=0.992$ ). In computing surface fluxes of momentum, 
heat, and moisture from bulk formulae (see Surface Fluxes), the surface wind is assigned the same value as at this lowest model level. The surface air temperature and specific humidity are determined from a surface heat balance equation and from surface wetness and soil moisture. See also Diffusion, Surface Characteristics, and Land Surface Processes.

\section{Orography}

$1 \times 1$-degree topography of Gates and Nelson (1975) is smoothed with a special filter (cf. Hoskins 1980) and truncated at the model's spectral T30 resolution (see Horizontal Resolution). High-resolution ( $6 \times 6$ minutes arc) topographic data are used for computation of orographic variances that are required for the gravity-wave drag parameterization (see Gravity-wave Drag).

\section{Ocean}

AMIP monthly sea surface temperatures are prescribed, with daily values determined by linear interpolation.

\section{Sea Ice}

AMIP monthly sea ice extents are prescribed. Ice thickness is specified from the climatology of Bourke and Garrett (1987) and Jacka (1983), and snow is allowed to accumulate on sea ice (see Snow Cover). A two-layer scheme is used for predicting the temperatures of sea ice/snow layers from the surface energy balance (see Surface Fluxes) with the inclusion of a heat flux from the ocean below.

\section{Snow Cover}

Precipitation falls as snow if the surface air temperature is $<273 \mathrm{~K}$. Snow thickness is determined from the prognostic value of snow mass and density (assumed to be $200 \mathrm{~kg} \mathrm{~m}^{-3}$ ); fractional coverage of a grid box by snow is not allowed. Snow cover affects the surface albedo of land and of sea ice (see Surface Characteristics), as well as the soil heat conductivity (see Land Surface Processes). Snowmelt contributes to soil moisture (see Land Surface Processes), and sublimation of snow is included in the surface evaporative flux (see Surface Fluxes). 
Surface Characteristics

- Different surface types (land/ocean/ice) may coexist in a grid box, and composite averages of surface quantities are computed in these cases (see Surface Fluxes and Land Surface Processes).

- The roughness length over land is a prescribed function of orography and vegetation type (cf. Louis 1984). Over ice surfaces, the roughness length is uniformly specified as $0.01 \mathrm{~m}$. Over the oceans, the dependence of roughness length on surface wind stress follows Charnock (1955), with a dimensionless factor of 0.018 (cf. Ariel and Murashova 1981). For a surface wind speed $<1 \mathrm{~m} \mathrm{~s}^{-1}$, a minimum ocean roughness is determined from an asymptotic relationship (cf. Zilitinkevich 1970).

- Annual mean surface albedos are prescribed for bare soil and vegetation after data of Wilson and Henderson-Sellers (1985). The ocean surface albedo is 0.06 , independent of solar zenith angle. The albedo of glacial ice on Greenland and Antarctica is 0.80 , while the albedo of sea ice is a function of surface temperature (cf. Wilson and Mitchell 1987). The albedo of snow-covered land depends on the annual mean background albedo, the liquid snow equivalent, and the maximum snow albedo.

- Longwave emissivity is prescribed as unity (blackbody emission) for all surfaces.

\section{Surface Fluxes}

- Shortwave absorption is determined from surface albedos, and long wave emission from the Planck equation with emissivity of 1.0 (see Surface Characteristics).

- Simulation of the vertical turbulent exchange of heat, moisture, and momentum is based on Monin-Obukhov similarity theory. The values of the momentum, heat, and moisture fluxes over an inhomogenous surface are area-weighted averages of the flux from each surface type in the grid box (see Surface Characteristics), and the surface drag and transfer coefficients depend on roughness length and vertical stability (cf. Louis 1979). The surface moisture flux includes sublimation from snowcovered surfaces; it also depends on the evapotranspiration efficiency $\beta$, which is prescribed as unity over ocean, snow, and ice surfaces, but which over land is a function of soil moisture (see Land Surface Processes).

- Time integration of the vertical turbulence equations is performed using the Euler-backward scheme with splitting (see Time Integration Schemes). 


\section{Land Surface Processes}

- Soil temperature is computed by a three-layer model with thicknesses $0.1,0.9$, and $2.0 \mathrm{~m}$, from top to bottom. The thickness of the top soil layer $(0.1 \mathrm{~m})$ is increased when snow accumulates (see Snow Cover). The thermodynamic properties of this layer are calculated as thickness-weighted averages of those of the two media; otherwise, the properties of the soil are assumed to be spatially uniform. When snow melts, the surface temperature remains constant $(273 \mathrm{~K})$ until all the snow disappears. Heating of the soil is computed from an energy-balance equation with the net surface heat fluxes (see Surface Fluxes) as the upper boundary condition and with zero heat flux assumed at the lower boundary of the bottom soil layer (at $3 \mathrm{~m}$ depth).

- Soil moisture is prognostically determined in two layers, with thicknesses the same as those of the two upper layers of the soil heat model $(0.1$ and $0.9 \mathrm{~m})$. Moisture from precipitation and snowmelt can accumulate in either layer, and can diffuse upward from the bottom layer (with constant diffusion coefficient $2.5 \times 10^{-6} \mathrm{~m}^{2} \mathrm{~s}^{-1}$ ). The hydraulic conductivity is computed by the method of Milly and Eagleson (1982). The field capacity for moisture in the upper layer is a function of soil and vegetation types (cf. Wilson and Henderson-Sellers 1985), and soil moisture is depleted by evapotranspiration and runoff. The evapotranspiration efficiency $\beta$ (see Surface Fluxes) depends on the ratio of soil moisture to the spatialiy uniform field capacity. Following Warrilow et al. (1986), the surface runoff from the upper layer is a function of the precipitation type (large-scale or convective), while runoff from the lower layer is computed assuming that its moisture holding capacity cannot exceed the field capacity (cf. Meleshko et al. 1991). 


\title{
MPI: Max-Planck-Institut fuer Meteorologie
}

\author{
AMIP Representative(s) \\ Dr. Erich Roeckner and Mr. Ulrich Schlese (of Deutsches Klimarechenzentrum), \\ Max-Planck-Institut fuer Meteorologie, Bundesstrasse 55, D-20146 Hamburg, \\ Germany; Phone: +49-40-41173-368 (Roeckner) or +49-40-41173-364 (Schlese); \\ Fax: +49-40-41173-366; e-mail: roeckner@dkrz.d400.de and schlese@dkrz.d400.de
}

\section{Model Designation \\ MPI ECHAM3 T42L19}

\section{Model Lineage}

This is the third in a series of models developed at MPI that derive from an earlier version (cycle 17) of the operational forecast model of the European Centre for Medium-Range Weather Forecasts (ECMWF). The present MPI model retains some features (more in numerics and dynamics than in physics) of the ECMWF model.

\section{Model Documentation}

The main documentation of the MPI model is given by Deutsches Klimarechenzentrum (DKRZ) Modellbetreuungsgruppe (1992)--hereafter DKRZ (1992). The results of changes in model resolution and physics are described by Roeckner et al. (1992).

\section{Numerical/Computational Properties}

\section{Horizontal Representation}

Spectral (spherical harmonic basis functions) with transformation to a Gaussian grid for calculation of nonlinear quantities and some physics.

\section{Horizontal Resolution}

Spectral triangular 42 (T42), roughly equivalent to $2.8 \times 2.8$ degrees latitudelongitude. 


\section{Vertical Domain}

Surface to $10 \mathrm{hPa}$. For a surface pressure of $1000 \mathrm{hPa}$, the lowest atmospheric level is at a pressure of about $996 \mathrm{hPa}$.

\section{Vertical Representation}

Hybrid sigma-pressure coordinates after Simmons and Burridge (1981) and Simmons and Struefing (1981).

\section{Vertical Resolution}

There are 19 irregularly spaced hybrid levels. For a surface pressure of $1000 \mathrm{hPa}$, five levels are below $800 \mathrm{hPa}$ and seven levels are above $200 \mathrm{hPa}$.

\section{Computer/Operating System}

The AMIP simulation was run on a Cray 2 computer using a single processor in the UNICOS environment.

\section{Computational Performance}

For the AMIP experiment, about 8 minutes of Cray 2 computation time per simulated day.

\section{Initialization}

For the AMIP simulation, the model atmosphere is intialized from the ECMWF analysis for 1 January 1979, and the soil moisture and snow cover/depth from the ECMWF January climatology.

\section{Time Integration Scheme(s)}

The semi-implicit time itegration scheme of Robert et al. (1972) and Robert $(1981,1982)$ is applied with an Asselin (1972) frequency filter. The time step is 24 minutes for dynamics and physics, except for radiation which is calculated at 2-hour intervals.

\section{Smoothing / Filling}

Orography is smoothed (see Orography). Negative moisture values arising from truncation of the spherical harmonic basis functions are filled for purposes of the 
radiation calculations, but negative moisture values are tolerated in transport algorithms (advection, convection, and diffusion). Negative cloud water values are avoided by invoking a suitable condensation term (see Cloud Formation).

\section{Sampling Frequency}

For the AMIP simulation, the model history is written every 6 hours.

\section{Dynamical/Physical Properties}

\section{Atmospheric Dynamics}

Primitive-equation dynamics are expressed in terms of vorticity, divergence, temperature, and the logarithm of surface pressure, specific humidity, and cloud water (a model prognostic variable). Virtual temperature is also used, where applicable, for diagnostic variables.

\section{Diffusion}

- Scale-selective fourth-order $\left(\nabla^{4}\right)$ horizontal diffusion is applied to all atmospheric prognostic variables for total spectral wave numbers $>15$ after the method of Laursen and Eliasen (1989). To avoid unrealistic results near mountains, horizontal diffusion operates only on the deviation of the temperature field from that of a standard atmosphere. The horizontal diffusion coefficient varies for different variables and vertical levels, and is selected to make the slope of the atmospheric spectral kinetic energy approximate that observed.

- Vertical diffusion operates above the planetary boundary layer (PBL) only in conditions of static instability. In the PBL, vertical diffusion of momentum, heat, cloud water, and moisture is proportional to the vertical gradient of the appropriate field. The vertically variable diffusion coefficient depends on stability (expressed as a bulk Richardson number) and the vertical shear of the u-wind, following standard mixing-length theory. See also Planetary Boundary Layer and Surface Fluxes.

\section{Gravity-wave Drag}

Drag associated with orographic gravity waves is simulated after the method of Palmer et al. (1986), as modified by Miller et al. (1989), using directionally dependent 
subgrid-scale orographic variances obtained from the U.S. Navy dataset (cf. Joseph 1980). Surface stress due to gravity waves excited by stably stratified flow over irregular terrain is calculated from linear theory and dimensional considerations. Gravity-wave stress is a function of atmospheric density, low-level wind, and the Brunt-Vaisalla frequency. The vertical structure of the momentum flux induced by gravity waves is calculated from a local wave Richardson number, which describes the onset of turbulence due to convective instability and the turbulent breakdown approaching a critical level.

\section{Solar Constant/Cycles}

The solar constant is the AMIP-prescribed value of $1365 \mathrm{~W} \mathrm{~m}^{-2}$. Both seasonal and diurnal cycles in solar forcing are simulated.

\section{Chemistry}

The carbon dioxide concentration is the AMIP-prescribed value of $345 \mathrm{ppm}$. The ozone profile is determined from total ozone in a column (after data by London et al. 1976) and the height of maximum concentration (after data by Wilcox and Belmont 1977), and depends on pressure, latitude, longitude, and season. Radiative effects of water vapor and of three types of aerosol (oceanic, desert, urban) are also included (see Radiation).

\section{Radiation}

- The radiation schemes follow Hense et al. (1982), Eickerling (1989), and Rockel et al. (1991). Radiative transfer equations are based on the two-stream approximation of Kerschgens et al. (1978) and Zdunkowski et al. (1980), and are solved in 4 shortwave spectral intervals (with boundaries at $0.215,0.685,0.891,1.273$, and 3.580 microns) and in 6 longwave spectral intervals (with boundaries at 3.96, 7.98, 8.89, 10.15, 11.76, 20.10 , and 100 microns).

- Shortwave equations are expressed in terms of optical depth, single-scattering albedo, diffuse and direct backscattering parameters, and diffusivity factor; longwave equations (with scattering effects neglected) are expressed in terms of the Planck function, optical depth, and diffusivity factor. Absorbers determining shortwave and longwave optical depths include ozone, carbon dioxide, and water vapor (with continuum absorption included). Optical depths for diffuse shortwave and longwave 
absorption are calculated using coefficients derived from "exact" reference models. Effects of pressure broadening on longwave absorption are also treated.

- In the shortwave, optical depths for Rayleigh scattering are determined from the molecular cross section for each gas, and those for absorption and Mie scattering by ocean, desert, and urban aerosols are from data of Shettle and Fenn (1975). Single scattering albedo is derived from the optical depths for diffuse shortwave scattering/ absorption. At a given vertical level, the total optical depth for direct shortwave radiation (which is dependent on solar zenith angle) is obtained from linear superposition of optical depths for scattering/absorption by gases and aerosols summed over all the levels above.

- The shortwave optical depth for clouds is parameterized after the method of Stephens (1978b), and the single-scattering albedo and backscattering parameter follow Kerschgens et al. (1978b). Longwave emissivity is an exponential function of geometrical cloud thickness, prognostic cloud water content, and a mass absorption coefficient, following Stephens (1978b). For purposes of the radiation calculations, clouds in contiguous vertical layers are treated as fully overlapped, and as randomly overlapped otherwise. Cf. DKRZ (1992) for further details. See also Cloud Formation.

\section{Convection}

The mass-flux convective scheme of Tiedtke (1989) accounts for shallow, midlevel and penetrative convection, as well as the effects of cumulus-scale downdrafts. Stratocumulus convection is parameterized as an extension of the model's vertical diffusion scheme (cf. Tiedtke et al. 1988). The closure assumption for midlevel/penetrative convection is that large-scale moisture convergence determines the bulk cloud mass flux; for shallow convection, the mass flux is maintained instead by moisture from surface evaporation. Entrainment/detrainment of mass in convective plumes occurs both through turbulent exchange and organized inflow/ outflow. Momentum transport by convective circulations is also included, following Schneider and Lindzen (1976).

\section{Cloud Formation}

- The (stratiform) cloud-formation scheme is based on prognostic cloud water, following Sundqvist (1978), Roeckner and Schlese (1985), and Roeckner et al. (1991). Subgrid-scale condensation and cloud formation in a fraction $\mathrm{c}$ of each grid box are 
governed by transport equations for water vapor and cloud water. The threshold relative humidity of the cloud-free fraction $(1-c)$ is prescribed as a function of height and stability: it is a minimum of 50 percent at tropopause level when penetrative convection occurs (cf. Xu and Krueger 1991); otherwise the threshold humidity decreases linearly from 99 percent at the surface to 85 percent at the top of the PBL (see Planetary Boundary Layer), above which it remains constant.

- Cloud droplets grow by condensation if the grid-mean relative humidity exceeds this threshold relative humidity or if the relative humidity in the c fraction of the grid box exceeds 100 percent. The condensation rate depends on the moisture convergence into the grid box, with a fraction $\mathrm{c}$ of the convergence producing more cloud, and the fraction ( 1 - c) increasing the relative humidity of the cloud-free part of the grid box.

- The cloud water in the cloud-free fraction $(1-c)$ that increases (decreases) as a result of advective/diffusive transports into the grid cell or through numerical effects (e.g., spectral truncation) is assumed to evaporate (condense) instantaneously; explicit filling of negative cloud water values is therefore unnecessary (see Smoothing / Filling). Convective cloud water is not advected (rather, there is instantaneous precipitation and/or evaporation to the environment--see Precipitation). Cf. DKRZ (1992) for further details. See also Radiation for treatment of cloud-radiative interactions.

\section{Precipitation}

- For convective precipitation, freezing and melting processes are not considered. No cloud water is stored in convective cloud (see Cloud Formation); once detrained, it evaporates instantaneously, with any portion not moistening the environment falling out as precipitation. Conversion from convective cloud droplets to rain/snow is proportional to the product of cloud water content and upward convective mass flux at cloud base, weighted by an empirical function of height (cf. Yanai et al. 1973). Convective snow forms if the temperature of the cloud layer is $<0^{\circ} \mathrm{C}$. Following Kessler (1969), evaporation of convective precipitation is assumed to be proportional to the saturation deficit and the rainfall intensity.

- For stratiform mixed-phase precipitation formation (i.e., in a temperature range from 0 to $-40^{\circ} \mathrm{C}$ ), the ice and liquid phases are treated independently. Growth of cloud droplets (see Cloud Formation) to precipitating raindrops occurs by 


\section{MPI}

autoconversion, following the exponential relationship of Sundqvist (1978), and by collisions with larger drops, following the parameterization of Smith (1990a). Partitioning of cloud liquid vs ice is according to the temperature-dependent relation of Matveev (1984), and the loss of ice crystals by sedimentation follows Heymsfield (1977). Evaporation of stratiform precipitation in a layer below cloud is proportional to the saturation deficit, but cannot exceed the precipitation flux at the layer top. Stratiform snow forms if the cloud layer temperature is $<0^{\circ} \mathrm{C}$. Falling convective and stratiform snow melts if the temperature of a layer is $>2{ }^{\circ} \mathrm{C}$. Cf. DKRZ (1992) for further details. See also Snow Cover.

\section{Planetary Boundary Layer}

The PBL is typically represented by the first 5 vertical levels above the surface (see Vertical Resolution). The PBL top (usually at elevations $<2000 \mathrm{~m}$ ) is determined as the greater of the height predicted from Ekman theory vs a convective height that depends on the dry static energy in the vertical. Heat, moisture, cloud water, and momentum fluxes follow Monin-Obukhov similarity theory at the surface and standard mixing-length theory above the surface. See also Diffusion and Surface Fluxes.

\section{Orography}

Orography is obtained from the U.S. Navy dataset with resolution of 10 minutes arc on a latitude/longitude grid (cf. Joseph 1980). These data are smoothed using a Gaussian filter with radius of influence $50 \mathrm{~km}$. The resulting heights are transformed into spectral space and truncated at $\mathrm{T} 42$ resolution.

\section{Ocean}

AMIP monthly sea surface temperatures are prescribed, with daily values determined by linear interpolation.

\section{Sea Ice}

AMIP monthly sea ice extents are prescribed. The temperature of the upper 0.10 meter of sea ice is computed from energy fluxes at the surface (see Surface Fluxes) and from the ocean below. The ocean heat flux depends on the ice thickness and the difference between the temperature of the underlying ocean and that of the ice. Snow does not accumulate on sea ice. Cf. DKRZ (1992) for further details. See also Snow Cover. 


\section{Snow Cover}

Snow falls to the surface if air temperatures at levels below where it forms (see Precipitation) are $<2{ }^{\circ} \mathrm{C}$. Snow accumulates only on land to a depth that is determined prognostically from a budget equation and melts if the temperatures of the snow and of the uppermost soil layer exceed $0^{\circ} \mathrm{C}$ (see Land Surface Processes). Snow cover affects surface albedo (see Surface Characteristics) as well as heat transfer and (after melting) soil moisture. The fractional area of a grid box that is snow covered is given by the ratio of the water-equivalent snow depth to a critical value of $0.015 \mathrm{~m}$, with complete coverage if this depth is exceeded. Sublimation of snow is calculated as part of the surface evaporative flux (see Surface Fluxes).

\section{Surface Characteristics}

- The fraction of each grid box covered by vegetation is determined from Matthews (1983) $1 \times 1$-degree data, but is reduced when soil moisture available in the root zone becomes less than 40 percent of field capacity $(0.20 \mathrm{~m})$--see Land Surface Processes.

- The surface roughness length is prescribed as a uniform $1 \times 10^{-3}$ meter over sea ice; it is computed prognostically over open ocean from the surface wind stress by the method of Charnock (1955), but is constrained to be a minimum of $1.5 \times 10^{-5} \mathrm{~m}$. Over land, the roughness length is geographically prescribed as a blended function of the local orographic variance (cf. Tibaldi and Geleyn 1981) and of the vegetation (cf. Baumgartner et al. 1977) that is interpolated to the model grid; the logarithm of the local roughness length is then smoothed by the same Gaussian filter as is used for orography (see Orography)

- The annual-mean surface albedo is obtained from satellite data of Geleyn and Preuss (1983). Over land, this background albedo is altered by snow cover as a linear function of the ratio of the water-equivalent snow depth to a critical value $(0.01 \mathrm{~m})$. Albedos of snow (range 0.30 to 0.80 ), sea ice (range 0.50 to 0.75 ), and continental ice (range 0.6 to 0.8 ) vary as a function of surface temperature and forested area, as given by Robock (1980) and Kukla and Robinson (1980). The albedo of ocean is a constant 0.065 for diffuse radiation, while that for the direct beam depends on solar zenith angle, but never exceeds 0.15 .

- Longwave emissivity is prescribed as 0.996 for all surfaces. Cf. DKRZ 1992 for further details. 


\section{Surface Fluxes}

- Surface solar absorption is determined from the surface albedo, and longwave emission from the Planck equation with prescribed uniform surface emissivity (see Surface Characteristics).

- Surface turbulent eddy fluxes of momentum, dry static energy (sensible heat), cloud water, and moisture are simulated as stability-dependent bulk formulae, following Monin-Obukhov similarity theory. The required near-surface values of wind, temperature, cloud water, and humidity are taken to be those at the lowest atmospheric level (sigma $=0.996$ ). (At the surface, cloud water is assumed to be zero.) Surface drag and transfer coefficients in the bulk formulae are functions of stability and roughness length (see Surface Characteristics), following Louis (1979) and Louis et al. (1981), but with modifications by Miller et al. (1992) for calm conditions over the oceans. The stability criterion is the moist bulk Richardson number, which includes the impact of cloud processes on buoyancy (cf. Brinkop 1992).

- The surface moisture flux depends on the surface specific humidity; over ocean, snow, ice, and wet vegetation fractions of each grid box, this is taken as the saturated humidity at the surface temperature and pressure (i.e., potential evaporation is assumed). Over the bare soil fraction, the surface specific humidity is the product of relative humidity (that is a function of soil moisture--see Land Surface Processes) and the saturated specific humidity. For a dry vegetation canopy, the potential evaporation is reduced by an evapotranspiration efficiency factor $\beta$ that is the inverse sum of aerodynamic resistance and stomatal resistance; the latter depends on radiation stress, canopy moisture, and soil moisture stress in the vegetation root zone (cf. Sellers et al. 1986, Blondin 1989, Blondin and Boettger 1987).

\section{Land Surface Processes}

- Soil temperature is determined after Warrilow et al. (1986) from the heat conduction in 5 layers (proceeding downward, layer thicknesses are 0.065, 0.254, $0.913,2.902$, and $5.70 \mathrm{~m}$ ), with net surface heat fluxes (see Surface Fluxes) as the upper boundary condition and zero heat flux as the lower boundary condition at $10 \mathrm{~m}$ depth.

- Snow pack temperature is also computed from the soil heat equation using heat diffusivity/capacity for ice in regions of permanent continental ice, and for bare 
soil where water-equivalent snow depth is $<0.025 \mathrm{~m}$. For snow of greater depth, the temperature of the middle of the snow pack is solved from an auxiliary heat conduction equation (cf. Bauer et al. 1985). The temperature at the upper surface is determined by extrapolation, but it is constrained not to exceed the snowmelt temperature of $0^{\circ} \mathrm{C}$.

- There are separate prognostic moisture budgets for snow, vegetation canopy, and soil reservoirs. Snow cover is augmented by snowfall and is depleted by sublimation and melting (see Snow Cover). Snow melts (augmenting soil moisture) if the temperatures of the snow pack and of the uppermost soil layer exceed $0{ }^{\circ} \mathrm{C}$. The canopy intercepts precipitation and snow (proportional to the vegetated fraction of a grid box), which is then subject to immediate evaporation or melting.

- Soil moisture is represented as a single-layer "bucket" model (cf. Manabe 1969) with field capacity $0.20 \mathrm{~m}$ that is modified to account for vegetative and orographic effects. Direct evaporation of soil moisture from bare soil and from the wet vegetation canopy, as well as evapotranspiration via root uptake, are modeled (see Surface Fluxes). Surface runoff includes effects of subgrid-scale variations of field capacity related to the orographic variance (see Orography); in addition, wherever the soil is frozen, moisture contributes to surface runoff instead of soil moisture. Deep runoff due to drainage processes also occurs independently of infiltration if the soil moisture is between 5 and 9 percent of field capacity (slow drainage), or is larger than 90 percent of field capacity (fast drainage). Cf. Duemenil and Todini (1992) for further details. 


\section{MRI: Meteorological Research Institute}

\section{AMIP Representative(s)}

Dr. Akio Kitoh and Dr. Tatsushi Tokioka, Meteorological Research Institute, 1-1, Nagamine, Tsukuba-shi, Ibaraki-ken, 305 Japan; Phone: +81-298-53-8594; Fax: +81-298-55-2552; e-mail: kitoh@mri-jma.go.jp

\section{Model Designation}

MRI GCM-II 4x5L15

\section{Model Lineage}

The MRI model is derived from an earlier version of the University of California at Los Angeles (UCLA) model (cf. Arakawa and Mintz 1974 and Arakawa and Lamb 1977). Subsequent modifications include increases in vertical resolution, and changes in parameterizations of gravity-wave drag, atmospheric radiation, convection, surface characteristics, and land surface processes.

\section{Model Documentation}

Documentation for an earlier five-level version of the model is provided by Tokioka et al. (1984). Descriptions of subsequent modifications are given by Yagai and Tokioka (1987), Yagai and Yamazaki (1988), Kitoh et al. (1988), Tokioka et al. (1988), Noda and Tokioka (1989), and Shibata and Aoki (1989).

\section{Numerical/Computational Properties}

\section{Horizontal Representation}

Finite differences on a C-grid with conservation of mass, momentum, energy, and potential enstrophy (cf. Arakawa and Lamb 1977).

\section{Horizontal Resolution}

$4 \times 5$-degree latitude-longitude grid. 


\section{Vertical Domain}

Surface to $1 \mathrm{hPa}$ (with the highest prognostic level at $1.39 \mathrm{hPa}$ ). For a surface pressure of $1000 \mathrm{hPa}$, the lowest atmospheric level is at a pressure of about $912 \mathrm{hPa}$. In addition, the depth of the boundary layer is a prognostic variable--see Planetary Boundary Layer.

\section{Vertical Representation}

Finite differences in hybrid coordinates. Above $100 \mathrm{hPa}$ log-pressure coordinates are used, and below $100 \mathrm{hPa}$ modified sigma coordinates: sigma $=(\mathrm{P}-\mathrm{PI}) /(\mathrm{PS}-\mathrm{PI})$, where $\mathrm{P}$ and PS are atmospheric and surface pressure, respectively, and PI is a constant $100 \mathrm{hPa}$. The vertical differencing scheme is after Tokioka (1978).

\section{Vertical Resolution}

15 hybrid levels. For a surface pressure of $1000 \mathrm{hPa}, 1$ level is below $800 \mathrm{hPa}$ and 9 levels are above $200 \mathrm{hPa}$. See also Vertical Representation.

\section{Computer/Operating System}

The AMIP simulation was run on a HITAC S-810/10 using a single processor in the VOS3 environment.

\section{Computational Performance}

For the AMIP experiment, about 6 minutes of HITAC S810/10 computation time per simulated day.

\section{Initialization}

For the AMIP simulation, a one-month "warm-up" period precedes the formal start on 1 January 1979. The model atmosphere, soil moisture, and snow cover/depth are initialized from a previous model solution for 1 December; then the model is integrated to a simulated 1 January state.

\section{Time Integration Scheme(s)}

The model is integrated by the leapfrog scheme with a time step of 6 minutes, and with a Matsuno step (an Euler forward integration followed by a backward 


\section{MRI}

integration) inserted hourly. At the forward stage of the Matsuno step, diabatic and dissipative terms, sources and sinks in atmospheric water vapor, and the change in the depth of the prognostic planetary boundary layer (PBL) are calculated. The PBL entrainment rate and the turbulent fluxes at the PBL top and at the surface are solved iteratively by backward implicit differencing (see Planetary Boundary Layer and Surface Fluxes). Shortwave and longwave radiation are computed hourly, but with transmission functions for longwave fluxes calculated every 3 hours.

\section{Smoothing / Filling}

Orography is area-averaged (see Orography). A longitudinal smoothing of the zonal pressure gradient and of the zonal and meridional mass flux is also performed in high latitudes (cf. Tokioka et al. 1984). A precise determination of the horizontal flux of atmosphere moisture and use of vertical interpolation at half-levels prevents the occurrence of negative humidity values (cf. Tokioka et al. 1984), thereby avoiding the need for moisture filling.

\section{Sampling Frequency}

For the AMIP simulation, the model history is written every 6 hours.

\section{Dynamical/Physical Properties}

\section{Atmospheric Dynamics}

Primitive-equation dynamics are expressed in flux form in terms of $u$ and $v$ winds, temperature, specific humidity, and surface pressure.

\section{Diffusion}

- Nonlinear horizontal diffusion is applied only to momentum (cf. Holloway and Manabe 1971).

- Vertical diffusion is not applied above the well-mixed PBL (see Planetary Boundary Layer). However, momentum is redistributed vertically by cumulus convection (see Convection). 


\section{Gravity-wave Drag}

Orographic gravity-wave drag is simulated following Palmer et al. (1986) with quantitative adjustments described by Yagai and Yamazaki (1988). The dependence of the surface momentum flux on the surface wind direction is considered (see Orography on the computation of the required subgrid-scale orographic covariances). Surface stress due to gravity waves excited by stably stratified flow over irregular terrain is calculated from linear theory and dimensional considerations. The gravity-wave stress is a function of atmospheric density, low-level wind, and the Brunt-Vaisalla frequency. The vertical structure of the momentum flux induced by gravity waves is calculated from a local wave Richardson number, which describes the onset of turbulence due to convective instability and the turbulent breakdown approaching a critical level.

\section{Solar Constant/Cycles}

The solar constant is the AMIP-prescribed value of $1365 \mathrm{~W} \mathrm{~m}^{-2}$. Both seasonal and diurnal cycles in solar forcing are simulated.

\section{Chemistry}

The carbon dioxide concentration is the AMIP-prescribed value of $345 \mathrm{ppm}$. Zonal profiles of ozone concentration are prescribed monthly from data of McPeters et al. (1984). The radiative effects of water vapor are also treated, but not those of aerosols (see Radiation).

\section{Radiation}

- Shortwave Rayleigh scattering and absorption in ultraviolet (wavelengths less than 0.35 micron) and visible (wavelengths 0.5 to 0.7 micron) spectral bands by ozone, and in the near-infrared (wavelengths 0.7 to 4.0 microns) by water vapor and carbon dioxide, follows the method of Lacis and Hansen (1974). Pressure corrections and multiple reflections between randomly overlapped partial clouds and the surface are treated.

- Longwave calculations are based on the multiparameter random model of Shibata and Aoki (1989) applied in four spectral regions (with boundaries at $2.0 \times 10^{3}$, $5.5 \times 10^{4}, 8.0 \times 10^{4}, 1.2 \times 10^{5}$, and $2.2 \times 10^{5} \mathrm{~m}^{-1}$ ). Absorption bands of carbon dioxide, 
ozone, and water vapor are included. Continuum absorption of water vapor is treated after the method of Clough et al. (1980), but with the temperature dependency used by Roberts et al. (1976). Mean diffuse transmittances in each region are determined from line-by-line calculations approximated by an exponential function including 10 or 12 parameters. Transmittances through inhomogeneous atmospheres are computed by a modified Godson (1953) method.

- In the shortwave, clouds are treated by a delta Eddington approximation with prescribed single-scattering albedo and asymmetry factor, and with cloud optical depth a function of height. In the longwave, clouds behave as blackbodies, except for high (above $400 \mathrm{hPa}$ ) clouds, whose emissivity is related to shortwave optical depth. For purposes of the radiation calculations, all clouds are assumed to be randomly overlapped in the vertical. See also Cloud Formation.

\section{Convection}

- Simulation of cumulus convection is by a modified Arakawa and Schubert (1974) scheme, as implemented by Lord (1978), Lord and Arakawa (1982), and Lord et al. (1982). Convective mass fluxes are assumed to originate in the PBL (see Planetary Boundary Layer); these are predicted from mutually interacting cumulus subensembles, which have different entrainment rates and levels of neutral buoyancy that define the tops of the clouds and their associated convective updrafts. In turn, the convective mass fluxes feed back on the large-scale fields of temperature (through latent heating and compensating subsidence), moisture (through precipitation and detrainment), and momentum (through cumulus friction). The effects on cloud buoyancy of phase changes from water to ice and the drying and cooling effects of convective-scale downdrafts on the environment are not included explicitly. The Arakawa-Schubert scheme is modified to impose an additional constraint between the minimum entrainment rate and the prognostic depth of the PBL (cf. Tokioka et al. 1988).

- The mass flux for each cumulus subensemble is predicted from an integral equation that includes a positive-definite work function (defined by the tendency of cumulus kinetic energy for the subensemble) and a negative-definite kernel which expresses the effects of other subensembles on this work function. The predicted mass fluxes are optimal solutions of this integral equation under the constraint that the rate of generation of conditional convective instability by the large-scale environment is balanced by the rate at which the cumulus subensembles suppress this instability 
via large-scale feedbacks (cf. Lord et al. 1982). The mass fluxes are computed by the "exact direct method," which guarantees an exact solution within roundoff errors.

- If the lapse rate becomes dry convectively unstable at any level, moisture and enthalpy are redistributed vertically. In addition, a moist convective adjustment simulates midlevel convection originating above the PBL. When the lapse rate exceeds moist adiabatic under supersaturated conditions, mass is mixed such that either the lapse rate is restored to moist adiabatic, or the supersaturation is eliminated by formation of convective precipitation. Cf. Tokioka et al. $(1984,1988)$ for further details.

\section{Cloud Formation}

- Five types of cloud are simulated: penetrative and midlevel convective cloud, cirrus anvil cloud, large-scale condensation cloud, and PBL stratus cloud. The PBL stratus is the only cloud type that does not interact with radiation.

- The penetrative and midlevel convective cloud are associated, respectively, with the cumulus convection and moist convective adjustment schemes (see Convection). Convective clouds in a vertical column are assumed to overlap randomly, with a total cloud fraction of 0.3 . Cirrus anvil cloud forms if cumulus convection penetrates to levels above $400 \mathrm{hPa}$, and large-scale condensation cloud is present if the local relative humidity of a layer is at least 100 percent. The latter two cloud types cover the grid box (cloud fracton =1).

- Stratus cloud capping the PBL forms if the specific humidity at the PBL top is greater than saturation (see Planetary Boundary Layer). The base of the cloud is determined from the vertical distribution of temperature and specific humidity in the PBL. See also Radiation for treatment of cloud-radiative interactions.

\section{Precipitation}

Large-scale precipitation results from condensation under supersaturated conditions. Precipitation from cumulus convection originating in the PBL is simulated by the Arakawa-Schubert (1974) scheme, and precipitation from midlevel convection by a moist adjustment process (see Convection). Both large-scale and convective precipitation may evaporate in falling through lower layers; the amount of evaporation is equal to that required to saturate each of these layers in turn. 
Planetary Boundary Layer

- The PBL is parameterized as a well-mixed layer after Randall (1976). The depth and mean structure of the PBL are prognostic functions of the surface momentum, heat, and moisture fluxes (see Surface Fluxes), as well as horizontal mass convergence, entrainment, and cumulus mass fluxes determined by the cumulus convection scheme (see Convection). PBL u-v winds, temperature, and specific humidity are predicted. Discontinuities in atmospheric variables that may exist at the PBL top because of the absence of vertical diffusion in the free atmosphere (see Diffusion) are also predicted. In addition, PBL stratus cloud is produced under saturated conditions (see Cloud Formation), and its evaporation by drier entrained air is treated.

- The turbulence kinetic energy (TKE) also is determined. (TKE is generated by wind shear and convective buoyancy fluxes, and is depleted by surface dissipation, by work done against the free atmosphere, and by newly turbulent air that is entrained into the PBL.) Because of the mutual dependence of the entrainment rate and the turbulent fluxes at the PBL top and at the surface, these quantities are solved iteratively under the assumption that the generation of TKE balances its dissipation. See also Surface Characteristics and Time Integration Schemes.

\section{Orography}

Orography, obtained from UCLA, is area averaged over each $4 \times 5$-degree model grid box. Orographic covariances required for the parameterization of gravity-wave drag (see Gravity-wave Drag) are computed from the U.S. Navy 10-minute resolution topography dataset (cf. Joseph 1980).

\section{Ocean}

AMIP monthly sea surface temperature fields are prescribed, with daily values determined by linear interpolation.

Sea Ice

- AMIP monthly sea ice extents are prescribed. Sea ice concentration fraction (ranging between 0 and 1 ) for each grid box is prescribed monthly by averaging over corresponding weekly U.S. Navy and National Oceanic and Atmospheric Administration (NOAA) Joint Ice Center data for 1979 to 1988 . The spatially variable thickness of sea ice is given by the local concentration fraction multiplied by 3.0 meters in the 
Northern Hemisphere and by 0.5 meter in the Southern Hemisphere. Daily values of the above quantities are determined by linear interpolation.

- The ice surface temperature is predicted from the net flux of energy (see Surface Fluxes), including a subsurface conduction heat flux that is proportional to the difference between the ice surface temperature and that prescribed $(271.3 \mathrm{~K})$ for the ocean below. Snow may accumulate on sea ice or melt if the snow surface temperature exceeds $0{ }^{\circ} \mathrm{C}$ (see Snow Cover). Snow also alters the heat capacity/conductivity of the ice, but the heat capacity of snow is independent of depth.

\section{Snow Cover}

If the surface air temperature is $<0{ }^{\circ} \mathrm{C}$, precipitation falls as snow. Snow may accumulate on both land and sea ice, with complete coverage of a grid box assumed (i.e., there is no fractional snow coverage). Prognostic snow mass is depleted both by snowmelt (which contributes to soil moisture) and by sublimation (which contributes to surface evaporation). Snow melts if its surface temperature exceeds $0{ }^{\circ} \mathrm{C}$. Snow cover affects both surface albedo and the thermal properties of the surface. See also Sea Ice, Surface Characteristics, Surface Fluxes, and Land Surface Processes.

\section{Surface Characteristics}

- The surface type is defined as open ocean, sea ice, glacial ice, lake, or land. (Lakes are treated as mixed layers of depth $10 \mathrm{~m}$, with prognostic temperatures.)

- The surface roughness length is a fixed value over oceans $\left(2 \times 10^{-4} \mathrm{~m}\right)$, sea ice $\left(1 \times 10^{-4} \mathrm{~m}\right)$, glacial ice $\left(5 \times 10^{-3} \mathrm{~m}\right)$, and land $(0.45 \mathrm{~m})$; the surface drag coefficient over land is a function of orographic variances, however (see Orography and Surface Fluxes).

- Surface albedos depend on solar zenith angle (cf. Paltridge and Platt 1976), but not on spectral interval. The ocean albedo is a maximum of 0.07 . The albedo of bare sea ice ranges between a maximum of 0.50 to 0.64 as a function of surface temperature; the albedo of snow-covered sea ice is a maximum of 0.70 , depending on snow depth. Snow-covered glacial ice albedos range between 0.70 and 0.85 . Snow-free land albedos are obtained from the data of Matthews (1983); with snow cover, the land albedo ranges from its snow-free value to a maximum between 0.60 to 0.70 , depending 
on topographic height (see Orography). On all surfaces, the albedo of melting snow is 0.60 , and that of frost is 0.30 .

- Longwave emissivity is prescribed as unity (blackbody emission) for all surfaces.

\section{Surface Fluxes}

- Surface solar absorption is determined from albedos, and longwave emission from the Planck equation with emissivity of 1.0 (see Surface Characteristics).

- Turbulent eddy fluxes of momentum, heat, and moisture are parameterized as bulk formulae with drag and transfer coefficients that depend on vertical stability (bulk Richardson number) and the (locally variable) depth of the PBL normalized by the surface roughness length, following Deardorff (1972). The drag coefficient over land is also increased as a function of orographic variances (see Orography and cf. Yagai and Tokioka 1987). The surface atmospheric values of wind, dry static energy, and humidity required for the bulk formulae are taken to be those predicted in the PBL (see Planetary Boundary Layer). Because of the mutual dependence of the turbulent fluxes at the surface and at the PBL top, these (as well as the PBL entrainment rate) are solved by mutual iteration. See also Surface Characteristics and Time Integration Scheme(s).

- The surface moisture flux depends also on an evapotranspiration efficiency factor $\beta$, which is set to unity over ocean and ice surfaces and in areas of continental dew formation; otherwise, over land, $\beta$ is a function of soil moisture (see Land Surface Processes).

\section{Land Surface Processes}

- Land-surface parameterizations follow Katayama (1978), with subsequent modifications in the treatment of soil moisture described by Kitoh et al. (1988). Soil heat and moisture move along the gradients of temperature and wetness, respectively. The thermodynamic and hydrologic effects of a vegetation canopy are not explicitly modeled, however.

- The temperature of bare and snow-covered land is predicted in four layers, and that of glacial ice in a single-layer. The upper boundary condition is the net balance of surface energy fluxes (see Surface Fluxes), while there is zero heat transfer at the lower boundary. Heat exchange associated with the freezing or melting of soil 
moisture and interstitial ice is taken into account. Snow cover and soil moisture/ice also affect the heat capacity/conductivity of the surface, but the heat capacity of snow is assumed to be independent of its depth.

- Soil moisture (in both liquid and frozen form) is predicted in four layers (with bottom boundaries at $0.10,0.50,1.50$, and $10.0 \mathrm{~m}$ below the surface); it is augmented by precipitation and snow/ice melt, and is depleted by surface evaporation and runoff. The evapotranspiration efficiency $\beta$ (see Surface Fluxes) is set to unity if the fractional soil moisture (the ratio of soil moisture to the field capacity, assumed to be 20 percent of the volume of a soil column) is at least $0.5 ; \beta$ is set to twice the fractional soil moisture otherwise. When all of the moisture in a soil layer is completely frozen (freezing begins when the layer temperature falls below $0{ }^{\circ} \mathrm{C}$ ), no deeper penetration of moisture is allowed. Runoff occurs in any layer that is either saturated or completely frozen. 


\section{NCAR: National Center for Atmospheric Research}

\section{AMIP Representative(s)}

Dr. David Williamson, National Center for Atmospheric Research, P.O. Box 3000, Boulder, Colorado 80307; Phone: +1-303-497-1372; Fax: +1-303-497-1324; e-mail: wmson@ncar.ucar.EDU.

\section{Model Designation}

NCAR CCM2 T42L18

\section{Model Lineage}

The NCAR Community Climate Model 2 (CCM2) is the historical descendant of the CCM1 model (cf. Williamson et al. 1987 and Hack et al. 1989), but with most dynamical and physical parameterizations qualitatively changed.

\section{Model Documentation}

Key documents are the NCAR CCM2 model description by Hack et al. (1993a) and the user's guide by Bath et al. (1992). Other papers that provide details on particular model features include Briegleb (1992), Briegleb et al. (1986), and Kiehl and Briegleb (1991) on the radiation parameterizations, Hack (1993) on the convection scheme, Holtslag and Boville (1993) on the simulation of boundary-layer diffusion, and Williamson and Rasch (1993) on the semi-Lagrangian transport scheme. Various aspects of the simulated climate with prescribed climatological sea surface temperatures are described by Kiehl et al. (1993) and Hack et al. (1993b). Model datasets available for analysis at NCAR (including those from the AMIP simulation) are summarized by Williamson (1993).

\section{Numerical/Computational Properties}

\section{Horizontal Representation}

Spectral (spherical harmonic basis functions) with transformation to a Gaussian grid for calculation of nonlinear quantities and most of the physics. Advection of water vapor is via shape-preserving semi-Lagrangian transport (SLT) on the Gaussian grid (cf. Williamson and Rasch 1993). 


\section{Horizontal Resolution}

Spectral triangular 42 (T42), roughly equivalent to $2.8 \times 2.8$ degrees latitudelongitude.

\section{Vertical Domain}

Surface to $2.917 \mathrm{hPa}$. For a surface pressure of $1000 \mathrm{hPa}$, the lowest atmospheric level is at a pressure of about $992 \mathrm{hPa}$.

\section{Vertical Representation}

Finite differences in hybrid sigma-pressure coordinates after Simmons and Struefing (1981), but modified to allow an upper boundary at nonzero $(2.917 \mathrm{hPa})$ pressure. The vertical-differencing formulation conserves global total energy in the absence of sources and sinks. See also Vertical Domain and Vertical Resolution.

\section{Vertical Resolution}

There are 18 unevenly spaced hybrid sigma-pressure levels. For a surface pressure of $1000 \mathrm{hPa}, 4$ levels are below $800 \mathrm{hPa}$ and 7 levels are above $200 \mathrm{hPa}$.

\section{Computer/Operating System}

The AMIP simulation was run on Cray 2 computers using multiple processors in the UNICOS environment.

\section{Computational Performance}

For the AMIP experiment, about 7 minutes on a single processor of the Cray 2 computer per simulated day.

\section{Initialization}

For the AMIP simulation, the model atmosphere is initialized from a previous model solution for 10 December 1978, and is then "spun up" to a simulated 1 January 1979 state. Snow cover/depth are prescribed from a mean January climatology only as a function of latitude (see Snow Cover). Annual-average ground wetness is prescribed for 10 surface types in place of specifying soil moisture (see Surface Characteristics and Land Surface Processes). 
Time Integration Scheme(s)

A centered semi-implicit time integration scheme (cf. Simmons et al. 1978) with an Asselin (1972) frequency filter is used for many calculations, but horizontal and vertical diffusion (see Diffusion), the advection of water vapor by the SLT scheme (see Horizontal Representation), and adjustments associated with convection and large-scale condensation (see Convection and Cloud Formation) are computed implicitly by a time-splitting procedure. The overall time step is 20 minutes for dynamics and physics, except for shortwave and longwave radiative fluxes and heating rates, which are calculated hourly (with longwave absorptivities and emissivities updated every 12 hours--see Radiation). Cf. Hack et al. (1993a) for further details.

\section{Smoothing / Filling}

Orography is smoothed (see Orography). Because advection of moisture is treated by the SLT scheme (see Horizontal Representation) negative specific humidity values are avoided. In cases where negative mixing ratios would result from application of the countergradient term in the parameterization of nonlocal vertical diffusion of moisture in the planetary boundary layer (PBL) (see Diffusion, Planetary Boundary Layer, and Surface Fluxes), the countergradient term is not calculated. In addition, at each 20-minute time step a "fixer" is applied to the surface pressure and water vapor so that the global average mass and moisture are conserved (cf. Williamson and Rasch 1993).

\section{Sampling Frequency}

For the AMIP simulation, the model history is written every 6 hours.

\section{Dynamical/Physical Properties}

\section{Atmospheric Dynamics}

Primitive-equation dynamics are expressed in terms of vorticity, divergence, temperature, specific humidity, and the logarithm of surface pressure. Virtual temperature is used where applicable, and frictional/diffusive heating is included in the thermodynamic equation. 


\section{Diffusion}

- In the troposphere, linear biharmonic $\left(\nabla^{4}\right)$ horizontal diffusion (with coefficient $1 \times 10^{16} \mathrm{~m}^{4} \mathrm{~s}^{-1}$ ) is applied to divergence and vorticity on hybrid sigmapressure surfaces, and to temperature on first-order constant pressure surfaces (requiring that biharmonic diffusion of surface pressure also be calculated on the Gaussian grid). In the stratosphere linear second-order $\left(\nabla^{2}\right)$ diffusion is applied to the same variables at the top three levels (with diffusivities increasing with height from $2.5 \times 10^{5}$ to $7.5 \times 10^{5} \mathrm{~m}^{2} \mathrm{~s}^{-1}$ ). In the top model layer, diffusion is enhanced by a factor of $10^{3}$ on all spectral wave numbers that violate the Courant-FriedrichsLewy (CFL) numerical stability criterion, based on the maximum wind speed.

- Above the PBL (see Planetary Boundary Layer) a second-order, stabilitydependent local formulation of the vertical diffusion of momentum, heat, and moisture is adopted (cf. Smagorinsky et al. 1965). The mixing length is taken to be a constant $30 \mathrm{~m}$, and the diffusivity is as given by Williamson et al. (1987) for unstable and neutral conditions and by Holtslag and Beljaars (1989) for stable conditions. Above the surface layer, but within the PBL under unstable conditions, mixing of heat and moisture (but not of momentum) is formulated as nonlocal diffusion, following Holtslag and Boville (1993)--see Surface Fluxes.

- Horizontal and vertical diffusion are calculated implicitly via time splitting apart from the solution of the semi-implicit dynamical equations (see Time Integration Schemes).

\section{Gravity-wave Drag}

Orographic gravity-wave drag is parameterized after McFarlane (1987). The momentum drag is given by the vertical divergence of the wave stress, which is proportional to the product of the local squared amplitude of the gravity wave, the Brunt-Vaisalla frequency, and the component of the local wind that is parallel to the flow at a near-surface reference level. At this reference level, the wave amplitude is bound by the lesser of the subgrid-scale orographic variance (see Orography) or a wave-saturation value defined by the reference Froude number. Above this level, the gravity-wave stress is assumed to be constant with height (zero vertical divergence), except in regions of wave saturation, where the amplitude is obtained from the local Froude number. 


\section{Solar Constant/Cycles}

The solar constant is the AMIP-prescribed value of $1365 \mathrm{~W} \mathrm{~m}^{-2}$. Both seasonal and diurnal cycles in solar forcing are simulated over a repeatable solar year of exactly 365 days (i.e., leap years are not included).

\section{Chemistry}

The carbon dioxide concentration is the AMIP-prescribed value of $345 \mathrm{ppm}$. Monthly ozone volume mixing ratios derived by Chervin (1986) from analyses of Duetsch (1978) are linearly interpolated to obtain intermediate values every 12 hours. Radiative effects of oxygen and of water vapor, but not of aerosols, are also included (see Radiation).

\section{Radiation}

- Shortwave scattering/absorption is parameterized by the delta-Eddington approximation of Joseph et al. (1976) and Coakley et al. (1983) applied in 18 spectral intervals, as described by Briegleb (1992). (These include 7 intervals between 0.20 and 0.35 micron to capture ozone Hartley-Huggins band absorption and Rayleigh scattering; 1 interval between 0.35 to 0.70 micron to capture Rayleigh scattering and ozone Chappius-band and oxygen B-band absorption; 7 intervals between 0.70 and 5.0 microns to capture oxygen A-band and water vapor/liquid absorption; and 3 intervals between 2.7 and 4.3 microns to capture carbon dioxide absorption.) Following Slingo (1989), the shortwave optical properties of clouds for the delta-Eddington approximation (optical depth, single-scattering albedo, asymmetry factor) are specified for 4 spectral ranges (with boundaries at $0.25,0.69,1.19,2.38$, and 4.0 microns). These properties depend on the specified effective droplet radius (10 microns) and the liquid water path (LWP), which is a prescribed nonlinear function of latitude and height (cf. Kiehl et al. 1993).

- Longwave absorption by ozone and carbon dioxide is treated by a broad-band absorptance technique, following Ramanathan and Dickinson (1979) and Kiehl and Briegleb (1991). A Voigt line profile (temperature) dependence is added to the pressure broadening of the absorption lines. Absorption by water vapor (and its overlap with that of ozone and carbon dioxide) are modeled as in Ramanathan and Downey (1986). Longwave broad-band emissivity of clouds is a negative exponential function of LWP, with 
all clouds assumed to be randomly overlapped in the vertical. Cf. Hack et al. (1993a) for further details. See also Cloud Formation.

\section{Convection}

- If the atmosphere is moist adiabatically unstable, temperature/moisture column profiles are adjusted by a mass-flux convective parameterization (cf. Hack 1993). The scheme utilizes a three-layer model that provides for convergence and entrainment in the lowest subcloud layer, cloud condensation and rainout in the middle layer, and limited detrainment in the top layer. This scheme is applied by working upward from the surface on three contiguous layers, and shifting up successively one layer at a time until the whole column is stabilized.

- The parameterization is based on simplified equations for the three-layer moist static energy that include (among other terms) the convective mass flux, a "penetration parameter" $\beta$ (ranging between 0 and 1 ) that regulates the detrainment of liquid water, and temperature and moisture perturbations furnished by the PBL parameterization (see Planetary Boundary Layer, Diffusion, and Surface Fluxes). Other free parameters in the scheme include minimum values for $\beta$, for the vertical gradient of moist static energy, and for the depth of precipitating convection; a characteristic convective adjustment time scale; and a cloud-water to rain-water autoconversion coefficient. The parameter $\beta$ is determined by iteration, subject to constraints that it and the vertical gradient of moist static energy be at least their minimum values, that the convective mass flux be positive, and that the detrainment layer not be supersaturated. The profiles of convective mass flux, temperature, and moisture are then obtained, and the total convective precipitation rate is calculated by vertical integration of the convectivescale liquid water sink.

- If a layer in the stratosphere (i.e., at the top three vertical levels) is dry adiabatically unstable, the temperature is adjusted so that stability is restored under the constraint that sensible heat be conserved. Whenever two layers undergo this dry adjustment, the moisture is also mixed in a conserving manner. (In the model troposphere, vertical diffusion provides stabilizing mixing, and momentum is mixed as well--see Diffusion). If a layer is supersaturated but stable, nonconvective condensation and precipitation result (see Precipitation). 


\section{Cloud Formation}

- The cloud prediction formulation is as described by Kiehl et al. (1993). Cloud amount is diagnostically determined from relative humidity, vertical velocity, atmospheric stability, and the convective precipitation rate, following a modified Slingo (1987) approach. Convective cloud, layer cloud, and low-level marine stratus/stratocumulus cloud associated with temperature inversions are treated. Clouds may form everywhere except in the surface layer (centered at sigma $=0.992$ ). Nonconvective cloud below $700 \mathrm{hPa}$ is classified as low-level cloud, that between 400 and $700 \mathrm{hPa}$ as midlevel cloud, and that above $400 \mathrm{hPa}$ as high-level cloud.

- Convective cloud base and top are determined by the vertical extent of moist instability (see Convection). In each vertical column, the total fractional cloud amount is a logarithmic function of the convective precipitation rate, but is constrained to be between 0.2 and 0.8 . The convective cloud fraction in each layer is determined assuming the cloud is distributed randomly in the vertical. For subsequent diagnosis of the fractional amount of nonconvective cloud (see below), the layer relative humidity is reduced proportional to the fraction of convective cloud present.

- In regions of upward vertical motion, the fraction of low-level layer cloud is a quadratic function of the difference between the reduced relative humidity (see above) and a constant threshold value ( 90 percent). The fraction of midlevel and high-level layer cloud is a quadratic function of the difference between the reduced relative humidity and a threshold value that is a linear function of the squared Brunt-Vaisalla frequency (i.e., it is proportional to the vertical stability).

- The fraction of marine stratus/stratocumulus is a function of the strength of the associated low-level inversion and the reduced relative humidity. Cf. Hack et al. (1993a) for further details. See also Radiation.

\section{Precipitation}

Subgrid-scale precipitation is generated in unstable conditions by the moist convective scheme (see Convection). Grid-scale precipitation forms as a result of supersaturation under stable conditions. In this case, the moisture is adjusted so that the layer is just saturated, with the excess condensing as precipitation; the layer temperature is adjusted according to the associated latent heat release. (Moisture and temperature are mutually adjusted in two iterations.) Subsequent evaporation of falling precipitation is not simulated. Cf. Hack et al. (1993a) for details. 


\section{Planetary Boundary Layer}

The PBL height is determined by iteration at each 20-minute time step following the formulation of Troen and Mahrt (1986); the height is a function of the critical bulk Richardson number for the PBL, $\mathrm{u}-\mathrm{v}$ winds and virtual temperature at the PBL top, and the 10-meter virtual temperature, which is calculated from the temperature and moisture of the surface and of the lowest atmospheric level (at sigma $=0.992$ ) following Geleyn (1988). Within the PBL, there is nonlocal diffusion of heat and moisture after Holtslag and Boville (1993); otherwise (and under all conditions for momentum), properties are mixed by the stability-dependent local diffusion that applies in the model's free atmosphere. See also Diffusion and Surface Fluxes.

\section{Orography}

- Raw orography is obtained from the U.S. Navy dataset with resolution of 10 minutes arc on a latitude/longitude grid (cf. Joseph 1980). These data are areaaveraged to a $1 \times 1$-degree grid, interpolated to a T119 Gaussian grid, spectrally truncated to the model's T42 Gaussian grid, and then spectrally filtered to reduce the amplitude of the smallest scales.

- The subgrid-scale orographic variances required for the gravity-wave drag parameterization (see Gravity-wave Drag) are also obtained from the U.S. Navy dataset. For the spectral T42 model resolution, the variances are first evaluated on a $2 \times 2$-degree grid, assuming they are isotropic. Then the variances are binned to the T42 Gaussian grid (i.e., all values whose latitude and longitude centers fall within each Gaussian grid box are averaged together), and are smoothed twice with a 1-2-1 spatial filter. Values over ocean are set to zero.

\section{Ocean}

AMIP monthly sea surface temperature fields are prescribed, with intermediate values determined at every 20 -minute time step by linear interpolation.

\section{Sea Ice}

AMIP monthly sea ice extents are prescribed, with intermediate values determined at every 20 -minute time step by linear interpolation. The temperature of the ice is predicted by the same four-layer scheme as used for soil temperature (see Land Surface Processes), but with a fixed temperature $\left(-2^{\circ} \mathrm{C}\right)$ of the underlying ocean rather 
than a zero-flux condition, as the lower boundary condition. The four layer thicknesses are all $0.5 \mathrm{~m}$, and the ice density, heat capacity, and conductivity are specified uniform constants; however, daily snow cover that is prescribed from climatology (see Snow Cover) alters the thermodynamic properties and thickness of the top layer in proportion to the relative mass of snow and ice. Cf. Hack et al. (1993a) for further details.

\section{Snow Cover}

Snow cover (expressed as an equivalent depth of water) is prescribed as a function of latitude and longitude from the mean January and July data of Forderhase et al. (1980) that are bilinearly interpolated to the T42 Gaussian grid. Intermediate daily values are obtained by assuming a single-harmonic annual variation. Snow cover is prescribed on sea ice as well as land, and affects the albedo, the roughness and wetness, and the thermodynamics of the surface (see Surface Characteristics). In addition, sublimation of snow contributes to the surface evaporative flux (see Surface Fluxes); however, because ground wetness is prescribed, snowmelt does not affect soil hydrology. See also Sea Ice and Land Surface Processes.

\section{Surface Characteristics}

- Over land, surface wetness fractions, roughness lengths, and albedos are derived from the Matthews (1983) $1 \times 1$-degree, 32-type vegetation data set. These values are aggregated to the 10 surface types (including land ice) distinguished by the model and are averaged over the T42 Gaussian grid boxes.

- The prescribed annual-average wetness fractions for the 10 surface types range between 0.01 for deserts to 1.0 for open water and ice- and snow-covered surfaces. On land, the surface wetness is weighted by the local fractional area of snow, which depends both on snow depth and the surface roughness length (to account for uneven coverage of vegetation). The heat capacity and conductivity of six distinguished land-surface thermal types also depend on surface wetness. See also Sea Ice and Land Surface Processes.

- Over land, the surface roughness length ranges from $0.04 \mathrm{~m}$ for tundra to $1.0 \mathrm{~m}$ for evergreen forest. The roughness is a uniform $1 \times 10^{-4} \mathrm{~m}$ over ocean, and $0.04 \mathrm{~m}$ over ice surfaces (cf. Hack et al. 1993a for further details). 
- The snow-free land albedos are constants (independent of time or moisture conditions) for the 10 distinguished surfaces. These are composed of five quantities: the fraction of strong zenith-angle dependence and four surface albedos (for two zenith angles and spectral intervals 0.2 to 0.7 micron and 0.7 to 4.0 microns). The land albedo is altered by snow cover: it is an average of the background albedo and the snow albedo (which depends on surface temperature for the diffuse beam and on solar zenith angle for the direct beam) that is weighted by the fractional snow cover (see above). Over the oceans, surface albedos are prescribed to be 0.025 for the direct-beam (with sun overhead) and 0.06 for the diffuse-beam component of radiation; the directbeam albedo varies with solar zenith angle. The albedo of ice surfaces is a function of surface temperature. Cf. Briegleb et al. (1986), Briegleb (1992) and Dickinson et al. (1986) for further details.

- The longwave emissivity is set to unity (blackbody emission) for all surfaces.

\section{Surface Fluxes}

- Surface solar absorption is determined from surface albedo, and longwave emission from the Planck equation with prescribed surface emissivity of 1.0 (see Surface Characteristics).

- Turbulent vertical eddy fluxes of momentum, heat, and moisture are expressed as bulk formulae, following Monin-Obukhov similarity theory. The values of wind, temperature, and humidity required for the bulk formulae are taken to be those at the lowest atmospheric level (sigma $=0.992$ ), which is assumed to be within a constant-flux surface layer. The drag and transfer coefficients in the bulk formulae are functions of roughness length (see Surface Characteristics) and stability (bulk Richardson number), following the method of Louis et al. (1981) for neutral and unstable conditions, and Holtslag and Beljaars (1989) for stable conditions. The bulk formula for the surface moisture flux also includes a prescribed surface wetness fraction (see Surface Characteristics and Land Surface Processes) that determines the evaporation realized as a fraction of potential evaporation from a saturated surface.

- Above the surface layer, but within the PBL (see Planetary Boundary Layer) under unstable conditions, mixing of heat and moisture (but not of momentum) is formulated as nonlocal vertical diffusion by eddies with length scales of the order of the PBL depth (cf. Deardorff 1972). Under these conditions, a countergradient term that 
depends on the surface flux, a convective vertical velocity scale, and the PBL height is added to the eddy diffusivity coefficient of heat and moisture. Within the stable and neutral PBL (and under all conditions for momentum), the same stability-dependent local vertical diffusion as is utilized in the model's free atmosphere applies (see Diffusion). Cf. Holtslag and Boville (1993) for further details.

\section{Land Surface Processes}

- Soil temperature is determined from heat conduction in a four-layer model. Layer thicknesses vary spatially, depending on the penetration depth of solar forcing, which is a function of forcing period and of the heat capacity/conductivity specified for each of the 10 distinguished surface types (see Surface Characteristics). The thicknesses of the bottom three soil layers are specified according to the local penetration depth of solar forcing with periods of 1 day, 2 weeks, and 1 year respectively. The thickness of the top soil layer is specified so that the diurnal range and phase of the surface temperature compares well with observations cited by Bhumralkar (1975). The top layer's thickness and heat capacity/conductivity are altered by snow (see Snow Cover) in proportion to the relative masses of snow and soil; these thermodynamic properties are also a linear function of ground wetness fraction (see below). The heat conduction equation is solved by a backward implicit Crank-Nicholson numerical scheme (cf. Smith 1965 and Washington and Verplank 1986), with the net surface energy balance being the upper boundary condition (see Surface Fluxes), and with zero heat flux specified at the lower boundary.

- Soil moisture is not predicted, and precipitation and snowmelt therefore do not affect the land surface hydrology. Instead, constant wetness fractions are specified for the 10 distinguished land-surface types (see Surface Characteristics). These wetness fractions affect the heat capacity and conductivity of the soil (see above), and they constrain the magnitude of the surface evaporative flux (see Surface Fluxes). Cf. Hack et al. (1993a) for further details. 


\title{
NMC: National Meteorological Center
}

\begin{abstract}
AMIP Representative(s)
Dr. Huug van den Dool, Dr. Wesley Ebisuzaki, and Dr. Eugenia Kalnay, World Weather Building, 5200 Auth Road, Camp Springs, Maryland 20746; Phone: +1-301763-8155 (van den Dool), +1-301-763-8227 (Ebisuzaki), +1-301-763-8005 (Kalnay); Fax: +1-301-763-8395; e-mail: wd51hd@sgi15.wwb.noaa.gov (van den Dool) and wd51we@sun1.wwb.noaa.gov (Ebisuzaki).
\end{abstract}

\section{Model Designation}

NMC MRF T40L18

\section{Model Lineage}

The model used for the AMIP experiment is a research version of the 1992 operational NMC Medium-Range Forecast (MRF) model, which is a modified form of the model documented by the NMC Development Division (1988). The principal modifications since 1988 include changes in the treatment of cloud formation, horizontal diffusion, orography, and surface evaporation, as well as the introduction of an atmospheric mass-conservation constraint (cf. Pan 1990 and Kanamitsu et al. 1991).

\section{Model Documentation}

Comprehensive documentation of model features is provided by the NMC Development Division (1988). Subsequent model development is summarized by Kanamitsu (1989), Kanamitsu et al. (1991), and Kalnay et al. (1990). The model configuration for the AMIP experiment is described by Ebisuzaki and van den Dool (1993).

\section{Numerical/Computational Properties}

\section{Horizontal Representation}

Spectral (spherical harmonic basis functions) with transformation to a Gaussian grid for calculation of nonlinear quantities and physics. 


\section{Horizontal Resolution}

Spectral triangular 40 (T40), roughly equivalent to a $3 \times 3$ degrees latitudelongitude.

\section{Vertical Domain}

Surface to about $21 \mathrm{hPa}$. For a surface pressure of $1000 \mathrm{hPa}$, the lowest atmospheric level is at a pressure of about $995 \mathrm{hPa}$.

\section{Vertical Representation}

Finite-difference sigma coordinates.

\section{Vertical Resolution}

There are 18 unevenly spaced sigma levels. For a surface pressure of $1000 \mathrm{hPa}$, 5 levels are below $800 \mathrm{hPa}$ and 4 levels are above $200 \mathrm{hPa}$.

\section{Computer/Operating System}

The AMIP simulation was run on a Cray Y/MP computer using eight processors in a UNICOS environment.

\section{Computational Performance}

For the AMIP experiment, about 4 minutes Cray Y/MP computation time per simulated day.

\section{Initialization}

For the AMIP simulation, the model atmosphere is initialized from a 1 January 1979 NMC analysis. Nonlinear normal mode initialization (cf. Machenauer 1977) with inclusion of diabatic heating is also employed. Soil moisture and snow cover/ depth are initialized from NMC "Launcher" climatologies originally obtained from the Geophysical Fluid Dynamics Laboratory (GFDL).

\section{Time Integration Scheme(s)}

The main time integration is by a leapfrog semi-implicit (gravity and zonal advection of vorticity) scheme with an Asselin (1972) frequency filter. The time 
step is 30 minutes for computation of dynamics and physics, except for full calculation of atmospheric radiation once every 3 hours (but with corrections made at every time step for diurnal variations in the shortwave fluxes and in the surface upward longwave flux). A mass-correction time scheme (cf. Kanamitsu et al. 1991 and van den Dool and Saha 1993) insures approximate conservation of mass in long integrations. In the AMIP experiment, the global dry atmospheric mass is restored at the beginning of each simulated month, and water-mass forcing also is employed (cf. Ebisuzaki and van den Dool 1993 and van den Dool and Saha 1993).

\section{Smoothing / Filling}

Mean orographic heights on the Gaussian grid are specified (see Orography). Negative atmospheric moisture values are not filled.

\section{Sampling Frequency}

For the AMIP simulation, the model history is written every 6 hours.

\section{Dynamical/Physical Properties}

\section{Atmospheric Dynamics}

Primitive-equation dynamics are expressed in terms of vorticity, divergence, the logarithm of surface pressure, specific humidity, and virtual temperature.

\section{Diffusion}

- Scale-selective, second-order horizontal diffusion after Leith (1971) is applied to vorticity, divergence, virtual temperature, and specific humidity on quasi-constant pressure surfaces (cf. Kanamitsu et al. 1991).

- Stability-dependent vertical diffusion of momentum and moisture follows the approach of Miyakoda and Sirutis (1986).

\section{Gravity-wave Drag}

Gravity-wave drag is simulated as described by Alpert et al. (1988). The parameterization includes determination of the momentum flux due to gravity waves at the surface, as well as at higher levels. The gravity-wave drag (stress) is given by the 
convergence of the vertical momentum flux. The surface stress is a nonlinear function of the surface wind speed and the local Froude number, following Pierrehumbert (1987). Vertical variations in the momentum flux occur when the local Richardson number is less than 0.25 (the stress vanishes), or when wave breaking occurs (local Froude number becomes critical); in the latter case, the momentum flux is reduced according to the Lindzen (1981) wave saturation hypothesis. Modifications are made to avoid instability when the critical layer is near the surface, since the time scale for gravity-wave drag is shorter than the model time step. See also Time Integration Scheme(s) and Orography.

\section{Solar Constant/Cycles}

The solar constant is the AMIP-prescribed value of $1365 \mathrm{~W} \mathrm{~m}^{-2}$. Both seasonal and diurnal cycles in solar forcing are simulated.

\section{Chemistry}

The carbon dioxide concentration is the AMIP-prescribed value of $345 \mathrm{ppm}$. Seasonal climatological zonal profiles of ozone concentrations are specified from data of Hering and Borden (1965) and London (1962). (These Northern Hemisphere seasonal concentrations are also prescribed for the Southern Hemisphere in the corresponding season. The resulting global ozone profiles are linearly interpolated for intermediate time points.) Radiative effects of water vapor, but not those of aerosol, are also included (see Radiation).

\section{Radiation}

- Shortwave Rayleigh scattering and absorption in ultraviolet (wavelengths less than 0.35 micron) and visible (wavelengths 0.5 to 0.7 micron) spectral bands by ozone, and in the near-infrared (wavelengths 0.7 to 4.0 microns) by water vapor follows the method of Lacis and Hansen (1974). Absorption by carbon dioxide is after Sasamori et al. (1972). Pressure corrections and multiple reflections between clouds and the surface are treated.

- Longwave radiation follows the simplified exchange method of Fels and Schwarzkopf (1975) and Schwarzkopf and Fels (1991), with calculation over spectral bands associated with carbon dioxide, water vapor, and ozone. Schwarzkopf and Fels (1985) transmission coefficients for carbon dioxide, a Roberts et al. (1976) 
water vapor continuum, and the effects of water vapor-carbon dioxide overlap and of a Voigt line-shape correction are included. The Rodgers (1968) formulation is adopted for ozone absorption.

- In the shortwave, cloud reflectances/absorptances are prescribed according to cloud height and type. In the longwave, low and middle clouds emit as blackbodies, while high clouds are graybodies, with emissivities ranging from 0.60 equatorward of 30 degrees to 0.30 poleward of 60 degrees. For purposes of the radiation calculations, clouds are treated as randomly overlapped in the vertical. See also Cloud Formation.

\section{Convection}

- Penetrative convection is simulated following Kuo (1965) with modifications as described by Sela (1980). Convection occurs in the presence of large-scale moisture convergence accompanied by a moist unstable lapse rate under moderately high relative humidity conditions. The vertical integral of the moisture convergence determines the total moisture available for moistening vs heating (through precipitation formation) the environment. If the moisture convergence in the first several lowest layers of a vertical column exceeds a critical threshold (equivalent to $5.097 \times 10^{-8} \mathrm{~m}$ rainfall), convection is initiated. The convective base is taken to be either the second or third layer above the surface, depending upon where the equivalent potential temperature is larger. The base temperature and humidity are used to determine the level of free convection and the level at which parcels saturate.

- An unstable subcolumn is then defined which extends from the base layer to the first layer for which a moist adiabatically lifted air parcel is not warmer than the environment. Within this subcolumn, the departures of the temperature and specific humidity of a saturated parcel from the respective environmental profiles in each layer determine the fraction of the total available moisture contributed to latent heat release vs moistening of that layer; the temperature and humidity profiles are revised accordingly. Cf. Sela (1980) for further details.

- Following Tiedtke (1983), simulation of shallow (nonprecipitating) convection is parameterized as an extension of the vertical diffusion scheme (see Diffusion). 


\section{Cloud Formation}

- The formation of stratiform clouds that are associated with fronts and tropical disturbances follows Slingo (1987). These clouds are modeled in high, middle, and low domains, and their fractional coverage is computed as a quadratic function of layer relative humidity wherever this exceeds a threshold of 80 percent. Within each domain, the cloud top is chosen as the layer with the maximum relative humidity, and the cloud is only one layer thick. Low frontal cloud is absent in regions of grid-scale subsidence, but low stratocumulus cloud may form in a temperature inversion of at least $0.05 \mathrm{~K}$ per $\mathrm{hPa}$ that is capped by dry air. The stratocumulus fraction is determined by the same relative-humidity criterion as for low frontal cloud; these clouds may be more than one layer thick, but they are excluded from the surface layer (cf. Kanamitsu et al. 1991).

- The height of subgrid-scale convective cloud is determined by the level of nonbuoyancy for moist adiabatic ascent (see Convection). The convective cloud fraction is a function of the cloud top/bottom and of the precipitation rate. Anvil cirrus also forms if the convective cloud penetrates above $400 \mathrm{hPa}$ (cf. Slingo 1987). See also Radiation for treatment of cloud-radiative interactions.

\section{Precipitation}

- Precipitation is produced both from large-scale condensation and from the convective scheme (see Convection). The large-scale precipitation algorithm compares the predicted specific humidity with a modified saturation value that is a function of the temperature and pressure of a vertical layer (cf. Sela 1980). If the predicted humidity exceeds this threshold value, condensation occurs and the predicted temperature field is adjusted to account for the associated latent heat release.

- Precipitation from large-scale condensation (but not from convection) evaporates as it acts to progressively saturate lower layers. All precipitation penetrating the bottom atmospheric layer is allowed to fall to the surface without further evaporation. See also Snow Cover.

\section{Planetary Boundary Layer}

While in theory the model PBL can extend throughout the entire atmosphere, its main effects are typically felt at the first 5 levels above the surface (at sigma $=0.995,0.981$, $0.960,0.920$, and 0.856). See also Diffusion, Surface Characteristics, and Surface Fluxes. 


\section{Orography}

Raw orography obtained from the U.S. Navy dataset with resolution of 10 minutes arc (cf. Joseph 1980) is area-averaged on the T126 Gaussian grid of the NMC operational model, transformed to spectral space, and then truncated at the T40 AMIP model resolution. Orographic variances are also computed on the T40 Gaussian grid for use in the gravity-wave drag parameterization (see Gravity-wave Drag).

\section{Ocean}

AMIP monthly sea surface temperature fields are prescribed, with daily values determined by linear interpolation.

Sea Ice

AMIP monthly sea ice extents are prescribed. (Because the AMIP dataset does not specify sea ice interior to the continents, two points representing frozen lakes in the NMC operational model are specified as land points instead--cf. Ebisuzaki and van den Dool 1993.) The sea ice is assumed to have a constant thickness of $2 \mathrm{~m}$, and the ocean temperature below the ice is specified to be $271.2 \mathrm{~K}$. The surface temperature of sea ice is determined from an energy balance that includes the surface heat fluxes (see Surface Fluxes) and the heat capacity of the ice. Snow accumulation does not affect the albedo or the heat capacity of the ice.

\section{Snow Cover}

Precipitation falls as snow if a linear combination of ground temperature (weighted 0.35) and the temperature at the lowest atmospheric level (weighted 0.65) is $<0{ }^{\circ} \mathrm{C}$. Snow mass is determined prognostically from a budget equation that accounts for accumulation and melting. Snowmelt contributes to soil moisture and sublimation of snow is included in the surface latent heat flux. Snow cover affects the surface albedo of soil, but not that of sea ice. See also Sea Ice, Surface Characteristics, Surface Fluxes, and Land Surface Processes.

\section{Surface Characteristics}

- Roughness lengths over the ocean are determined from the surface wind stress after the method of Charnock (1955). Over sea ice, the roughness length is a 
uniform $1 \times 10^{-4} \mathrm{~m}$. Spatially varying roughness lengths over land are prescribed from data of Dorman and Sellers (1989) that includes 12 vegetation types.

- Over oceans, the surface albedo depends on zenith angle (cf. Payne 1972). The albedo of sea ice is a constant 0.50. Albedos for snow-free land are obtained from Dorman and Sellers (1989) data. Snow cover modifies the local background albedo of the land surface as follows. Poleward of 70 degrees latitude, permanent snow with albedo 0.75 is assumed. Equatorward of 70 degrees, the snow albedo is set to 0.60 if the water-equivalent snow depth is at least $0.01 \mathrm{~m}$; otherwise, the albedo is a linear combination of the background and snow albedos weighted by the fraction of snow cover in the grid box (see Snow Cover). Albedos do not depend on spectral interval.

- Longwave emissivity is prescribed to be unity (blackbody emission) for all surfaces.

\section{Surface Fluxes}

- Surface solar absorption is determined from the surface albedos, and longwave emission from the Planck equation with emissivity of 1.0 (see Surface Characteristics).

- In the lowest atmospheric layer surface turbulent eddy fluxes of momentum, heat, and moisture are expressed as bulk formulae, following Monin-Obukhov similarity theory as formulated by Miyakoda and Sirutis (1986). The momentum flux is proportional to the product of a drag coefficient, the wind speed, and the wind velocity vector at the lowest atmospheric level (sigma $=0.995$ ). Surface sensible heat flux is proportional to the product of an exchange coefficient, the wind speed at the lowest atmospheric level, and the vertical difference between the temperature at the surface and at the lowest level. The drag and transfer coefficients are functions of surface roughness length (see Surface Characteristics) and stability (bulk Richardson number).

- The surface moisture flux is given by the product of the potential evaporation and an evapotranspiration efficiency $\beta$. Potential evaporation is calculated by the Penman-Monteith method (cf. Monteith 1965) from the air temperature and humidity at the lowest level, and over land from a specified constant minimum stomatal resistance (cf. Pan 1990). Over oceans, snow, and ice surfaces, $\beta$ is prescribed to be unity, while over land it is a function of the ratio of soil moisture to the constant field capacity (see Land Surface Processes). 
Land Surface Processes

- Soil temperature is computed in three layers at depths of $0.1,0.5$, and $5.0 \mathrm{~m}$ by a fully implicit time integration scheme (cf. Miyakoda and Sirutis 1986). Soil heat capacity/conduction is modified by snow cover through its effect on soil moisture availability (i.e., evapotranspiration efficiency $\beta=1$ for snow-covered surfaces--see Surface Fluxes).

- Soil moisture is represented by the single-layer "bucket" model of Manabe (1969), with a uniform field capacity of $0.15 \mathrm{~m}$. Soil moisture is increased by snowmelt as well as precipitation; it is decreased by surface evaporation, which is determined from a product of the evapotranspiration efficiency $\beta$ and the potential evaporation (see Surface Fluxes). Over land, $\beta$ is determined from the ratio of local soil moisture to the field capacity, with runoff implicitly occurring if this ratio exceeds unity. 


\section{NRL: Naval Research Laboratory}

\section{AMIP Representative(s)}

Dr. Thomas Rosmond and Dr. Timothy Hogan, Prediction Systems, Naval Research Laboratory, Monterey, California, 93943-5006; Phone: +1-408-647-4736; Fax: +1-408-656-4769; e-mail: rosmond@helium.nrlmry.navy.mil

\section{Model Designation}

NRL NOGAPS3.2 T47L18

\section{Model Lineage}

The NRL model used for the AMIP experiment is version 3.2 of the Naval Operational Global Atmospheric Prediction System (NOGAPS) spectral model, which was first developed in 1988.

\section{Model Documentation}

Key documentation for the model is provided by Hogan and Rosmond (1991).

\section{Numerical/Computational Properties}

\section{Horizontal Representation}

Spectral (spherical harmonic basis functions) with transformation to a Guassian grid for calculation of nonlinear quantities and some physics.

\section{Horizontal Resolution}

Spectral triangular 47 (T47), roughly equivalent to $2.5 \times 2.5$-degrees latitudelongitude.

\section{Vertical Domain}

Surface to $1 \mathrm{hPa}$. For a surface pressure of $1000 \mathrm{hPa}$, the lowest atmospheric level is at a pressure of about $995 \mathrm{hPa}$. 


\section{Vertical Representation}

Modified hybrid sigma-pressure coordinates after Simmons and Struefing (1981), utilizing energy-conserving vertical differencing and averaging.

\section{Vertical Resolution}

There are 18 unevenly spaced hybrid levels. For a surface pressure of $1000 \mathrm{hPa}$, five levels are below $800 \mathrm{hPa}$ and five levels are above $200 \mathrm{hPa}$.

\section{Computer/Operating System}

The AMIP simulation was run on a Cray Y/MP computer using four processors in the UNICOS environment.

\section{Computational Performance}

For the AMIP experiment, about 10 minutes of Cray Y/MP computation time per simulated day.

\section{Initialization}

For the AMIP simulation, the model atmosphere is initialized from the ECMWF FGGE III-B analysis fields for 00Z on 1 January 1979, with nonlinear normal-mode initialization applied. Snow cover/depth is set initially to zero everywhere. Ground wetness values (see Land Surface Processes) are specified from the Fleet Naval Oceanographic Center (FNOC) climatological data for January (cf. FNOC 1986).

\section{Time Integration Scheme(s)}

A semi-implicit time integration scheme with a spectral filter (cf. Robert et al. 1972) is used for most quantities, but the zonal advection of the vorticity and the moisture function are calculated by a fully implicit method (cf. Simmons and Jarraud 1983). Turbulent surface fluxes and vertical diffusion (see Surface Fluxes and Vertical Diffusion) are also computed by implicit methods. The time step is 20 minutes for dynamics and physics, except for full calculation of radiative fluxes every 1.5 hours. 
NRL

Smoothing / Filling

Orography is smoothed (see Orography). Negative moisture values arising from the spectral truncation are filled by "borrowing" from positive-valued points at vertical levels below, with an artificial moisture flux provided from the ground if necessary.

\section{Sampling Frequency}

For the AMIP simulation, the model history is written every 6 hours.

\section{Dynamical/Physical Properties}

\section{Atmospheric Dynamics}

Primitive-equation dynamics are expressed in terms of vorticity, divergence, virtual potential temperature, surface pressure, and specific humidity.

\section{Diffusion}

- Fourth-order horizontal diffusion is spplied in spectral space on hybrid vertical levels to vorticity and divergence, and to departures of specific humidity and virtual

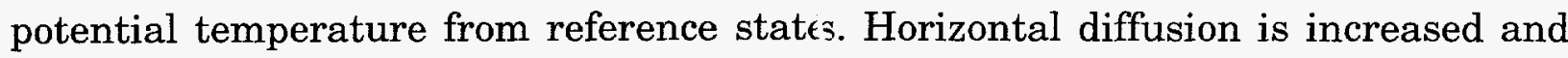
spectral tendencies are truncated at the urjer three vertical levels when the wind speed at the top level exceeds $120 \mathrm{~m} \mathrm{~s}^{-1}$.

- Vertical diffusion of momentum, heat, noisture, and buoyancy (virtual potential temperature) is parameterized by $\mathrm{K}$-theorv, with the mixing length a function of stability (bulk Richardson number) following te formulation of Louis et al. (1981).

\section{Gravity-wave Drag}

- Momentum transports associated with gr ity waves are simulated by a modified Palmer et al. (1986) method, using dirctionally dependent subgrid-scale orographic variances (see Orography). Surface str ss due to gravity waves excited by stably stratified flow over irregular terrain is clculated from linear theory and dimensional considerations. Gravity-wave stress is : function of atmospheric density, low-level wind, and the Brunt-Vaisalla frequenc. The vertical structure of the momentum flux induced by gravity waves is calculaid from a local wave Richardson 
number, which describes the onset of turbulence due to convective instability and the turbulent breakdown approaching a critical level.

- To allow more gravity-wave drag in the model's upper atmosphere, the Palmer et al. wave breakdown criteria are modified as follows. Below $450 \mathrm{hPa}$, the decrease in wave stress cannot exceed 50 percent of the stress in the layer below; above $450 \mathrm{hPa}$, the stress can be completely absorbed in any layer. However, if the projection of the orographically induced surface stress onto the wind velocity of a layer is zero, then the gravity-wave stress is set to zero in all layers above this critical layer.

\section{Solar Constant/Cycles}

The solar constant is the AMIP-prescribed value of $1365 \mathrm{~W} \mathrm{~m}^{-2}$. Both seasonal and diurnal cycles in solar forcing are simulated.

\section{Chemistry}

The carbon dioxide concentration is the AMIP-prescribed value of $345 \mathrm{ppm}$. Seasonal zonal profiles of ozone are prescribed from the data of Dopplick (1974), with daily values determined via a two-coefficient Fourier interpolation of the seasonal data. Radiative effects of water vapor are also included, but not those of aerosols (see Radiation).

\section{Radiation}

- Shortwave radiation calculations follow Davies (1982) and Lacis and Hansen (1974). Absorption by ozone and water vapor is calculated in two spectral bands: for wavelengths less than 0.9 micron (where ozone absorption is especially significant), and for wavelengths greater than 0.9 micron (only for water vapor). At wavelengths less than 0.9 micron, absorption calculations follow the Coakley and Chylek (1975) two-stream solution for diffuse radiation at several Rayleigh optical depths of both ozone and water vapor. For wavelengths greater than 0.9 micron, clear-sky absorption is treated by the approach of Lacis and Hansen (1974); the reflected and transmitted diffuse fluxes in cloudy skies are computed at each level for four different absorptions using both the Coakley and Chylek (1975) and the Sagan and Pollack (1967) two-stream solutions. Total fluxes and absorptions are obtained by combining diffuse fluxes at each level using the adding method of Liou (1980). Shortwave cloud parameters include an optical thickness that is a function of the temperature of the 
layer and the total cloud fraction (see Cloud Formation), and a single-scattering albedo that depends on the optical thickness as well as on the effective water-vapor content of the cloud.

- Longwave radiation is after Harshvardhan et al. (1987). Absorption calculations in 5 spectral intervals (wavenumbers between 0 and $3 \times 10^{6} \mathrm{~m}^{-1}$ ) follow the broadband transmission approach of Chou (1984) for water vapor, that of Chou and Peng (1983) for carbon dioxide, and that of Rodgers (1968) for ozone. Continuum absorption by water vapor is treated following Roberts et al. (1976). Cloud longwave emissivity varies linearly with temperature between a value of 0.50 for ice clouds (temperature $<233 \mathrm{~K}$ ) and a value of 1.0 for water clouds (temperature $>273 \mathrm{~K}$ ). For purposes of the radiation calculations, clouds are assumed to be a composite of both maximally overlapped and randomly overlapped elements. Cf. Hogan and Rosmond (1991) for further details.

\section{Convection}

- Penetrative convection is simulated by the method of Arakawa and Schubert (1974). The scheme predicts mass fluxes from mutually interacting cumulus subensembles having different entrainment rates and levels of neutral buoyancy that define the tops of the clouds and their associated convective updrafts. In turn, the predicted convective mass fluxes feed back on the large-scale fields of temperature (through latent heating and compensating subsidence), and moisture (through precipitation and detrainment). The implementation follows Lord et al. (1982), but with the following modifications: a simplified ice parameterization is used; 20 percent of the convective precipitation produced in a layer is allowed to evaporate as it falls; moist and evaporative downdraft terms are included in the cloud budget equations; and the latent heat of condensation is made a function of temperature.

- The cumulus mass flux for each subensemble is predicted from an integral equation involving a positive-definite work function and a negative-definite kernel which expresses the effects of other subensembles on this work function. The mass fluxes are positivedefinite optimal solutions of this integral equation under the constraint that the rate of generation of conditional convective instability by the large-scale environment is balanced by the rate at which the cumulus subensembles suppress this instability via large-scale feedbacks. A numerically efficient variational method (convergence usually within three iterations) is used to solve the integral equation.

- Shallow (stratocumulus) convection is parameterized as an extension of the model's vertical diffusion scheme (see Diffusion) after Tiedtke (1983). For shallow convection, the following conditions must be met: the relative humidity of the lowest layer is 
at least 70 percent; the ground temperature exceeds the surface air temperature; the lifted condensation level is within $175 \mathrm{hPa}$ of the surface, and there is moist adiabatic instability within this layer. The shallow convection layer extends from the surface to the top of the moist instability, with a maximum depth of $175 \mathrm{hPa}$. Cf. Hogan and Rosmond (1991) for further details. See also Cloud Formation and Precipitation.

\section{Cloud Formation}

- The amount of stratiform cloud is diagnosed following Slingo and Ritter (1985): at each vertical level, the cloud fraction is a quadratic function of the difference between the average relative humidity and a threshold value that depends on the sigma level.

- Cumulus (convective) cloud extends from the lifted condensation level to the highest cloud-top level predicted by the convective scheme (see Convection). The cloud fraction is a logarithmic function of the convective rainfall rate after Slingo (1987). It is taken to be a constant (maximum fraction 0.8) up to the level where anvil cloud is diagnosed (temperature $=233 \mathrm{~K}$ ). If the cloud-top temperature is $<233 \mathrm{~K}$, the cumulus cloud fraction is increased by 0.20 to account for the presence of ice anvils. See also Radiation for treatment of cloud-radiative interactions.

\section{Precipitation}

- The convective scheme produces precipitation, which may subsequently evaporate as it falls (see Convection).

- Any remaining supersaturation of a layer is removed in the formation of largescale precipitation by the saturation adjustment of Haltiner and Williams (1980). Working downward from the top layer, the adjustment is made with respect to water vapor for temperatures above $0^{\circ} \mathrm{C}$, and with respect to ice for temperatures below $-40^{\circ} \mathrm{C}$; for intermediate temperatures, a linear combination of these adjustments is applied. The associated latent heat release is also determined from a temperature-dependent linear combination of the heats of condensation and fusion. This large-scale precipitation may evaporate as it falls, producing supersaturation in lower layers. In that event, the adjustment is repeated downward to the bottom layer, where no evaporation of precipitation is allowed. The remaining precipitation falls to the surface as rain or snow. See also Cloud Formation and Snow Cover. 


\section{Planetary Boundary Layer}

The PBL is typically represented by the first five levels above the surface, but its depth is not explicitly determined. See also Diffusion, Surface Characteristics, and Surface Fluxes.

\section{Orography}

Model orography is derived from the U.S. Navy 10-minute resolution global terrain dataset (cf. Joseph 1980). The terrain heights are enhanced by the silhouette method, and then are transformed to the spectral representation and truncated at T47 resolution (see Horizontal Resolution). Spectral smoothing with a Lanczos (1956) filter is also applied to lessen the effects of negative terrain heights resulting from the spectral truncation. Orographic variances required by the gravity-wave drag parameterization (see Gravity-wave Drag) are obtained from the same dataset.

\section{Ocean}

AMIP monthly sea surface temperature fields are prescribed, with values determined at every time step by linear interpolation.

\section{Sea Ice}

AMIP monthly sea ice extents are prescribed. The temperature of the ice is predicted in a manner similar to that for soil (see Land Surface Processes) from a net energy balance, with relaxation to a climatological temperature of $272.2 \mathrm{~K}$ (the relaxation time constant is derived assuming a uniform ice thickness of $2 \mathrm{~m}$ ). Snow does not accumulate on sea ice.

\section{Snow Cover}

If the ground temperature is $<0{ }^{\circ} \mathrm{C}$, precipitation falls as snow (see Precipitation). Snow is allowed to accumulate on land only to a maximum water-equivalent depth of $0.1 \mathrm{~m}$. Snow cover alters the surface albedo (see Surface Characteristics) and thermodynamic properties of the surface (see Land Surface Processes), and sublimation of snow contributes to surface evaporation (see Surface Fluxes). If the ground temperature increases above freezing when snow is present, the amount of heat necessary to lower the ground temperature again to $0{ }^{\circ} \mathrm{C}$ is used to melt snow. This snowmelt does not contribute to soil moisture, however (see Land Surface Processes). 


\section{Surface Characteristics}

- The surface roughness length for ocean points is updated from the surface wind stress at each time step following the Charnock (1955) relation. For land and ice surfaces, the roughness lengths are specified from the FNOC (1986) monthly climatologies.

- Surface albedos, also specified from the FNOC (1986) climatologies, are a function of solar zenith angle, but not spectral interval. The albedo of snow-covered land varies linearly from its bare-ground value to a maximum of 0.84 when the snow depth within a grid box exceeds $0.01 \mathrm{~m}$ water equivalent.

- Longwave emissivity is prescribed as unity (blackbody emission) for all surfaces.

\section{Surface Fluxes}

- Surface solar absorption is determined from the surface albedos, and longwave emission from the Planck equation with emissivity of 1.0 (see Surface Characteristics).

- The surface fluxes of momentum, sensible heat, buoyancy (virtual potential temperature), and moisture are computed implicitly (see Time Integration Schemes) from bulk formulae with drag and transfer coefficients that are functions of static stability (bulk Richardson number) and roughness length, following Louis et al. (1981). The surface winds, temperatures, and humidities required for these bulk formulae are those at the lowest atmospheric level (at about $995 \mathrm{hPa}$ for a surface pressure of $1000 \mathrm{hPa}$ ). The surface fluxes also depend on the ground temperatures of ocean, sea ice, and land (see Ocean, Sea Ice, and Land Surface Processes).

- In addition, surface buoyancy and moisture fluxes depend on ground specific humidity, which is defined as a weighted linear combination of the humidity at the lowest atmospheric level and the saturated humidity at the ground temperature. The weights are determined from the ground wetness, which is set to unity over ocean, snow, and ice surfaces, but which over land is prescribed from monthly FNOC (1986) climatological estimates of soil moisture (see Land Surface Processes). Cf. Hogan and Rosmond (1991) for further details. 
NRL

\section{Land Surface Processes}

- Soil temperatures are computed from a surface energy balance that includes snowmelt (see Surface Fluxes and Snow Cover) as well as relaxation (with 100-hour time constant) to FNOC (1986) monthly climatological deep-ground temperatures. These deep temperatures are derived from observed surface atmospheric temperatures that are lagged by one month, with annual cycle reduced by 30 percent. The heat capacity specified for soil is also a nonlinear function of the ground wetness, and the thermal conductivity of snow-covered ground is set to about twice that of bare ground.

- Spatially varying ground wetness is prescribed from FNOC (1986) monthly climatological estimates of fractional soil moisture (ratio of soil moisture to a saturated value). Cf. Hogan and Rosmond (1991) for further details. 


\section{RPN: Recherche en Prévision Numérique}

\section{AMIP Representative(s)}

Dr. Harold Ritchie, Recherche en Prévision Numérique, 2121 Trans-Canada Highway, Room 500, Dorval, Quebec, Canada H9P 1J3: Phone: +1-514-421-4739; Fax: +1-514-421-2106; e-mail: hritchie@rpn.aes.doe.ca

\section{Model Designation \\ RPN NWP-D40P29 T63L23}

\section{Model Lineage}

The RPN model derives from research on application of the semi-Lagrangian method (cf. Ritchie 1985, 1986, 1987, 1988, 1991) and from physical parameterizations in use in other models at this institution.

\section{Model Documentation}

The semi-Lagrangian numerics are described by Ritchie (1991), and the finite element discretization by Beland and Beaudoin (1985). Descriptions of some physical parameterizations are provided by Benoit et al. (1989).

\section{Numerical/Computational Properties}

\section{Horizontal Representation}

Semi-Lagrangian spectral (spherical harmonic basis functions) with transformation to a Gaussian grid for calculation of nonlinear quantities and some physics.

\section{Horizontal Resolution}

Spectral triangular 63 (T63), roughly equivalent to $1.9 \times 1.9$ degrees latitudelongitude.

\section{Vertical Domain}

Surface to about $10 \mathrm{hPa}$. For a surface pressure of $1000 \mathrm{hPa}$, the lowest atmospheric level is at $1000 \mathrm{hPa}$ (using a nonstaggered vertical grid). 


\section{RPN}

\section{Vertical Representation}

Finite-element sigma coordinates. (Some changes in the form of the vertical discretization of the model equations are required to produce a formulation appropriate for use of the semi-Lagrangian method--cf. Ritchie 1991.)

\section{Vertical Resolution}

There are 23 unevenly spaced sigma levels. For a surface pressure of $1000 \mathrm{hPa}$, 7 levels are below $800 \mathrm{hPa}$ and 7 levels are above $200 \mathrm{hPa}$.

\section{Computer/Operating System}

The AMIP simulation was run on a NEC SX-3 computer using a single processor in a UNIX operating environment.

\section{Computational Performance}

For the AMIP experiment, about 4 minutes computation time per simulated day.

\section{Initialization}

For the AMIP simulation, the model atmosphere, soil moisture, and snow cover/ depth are initialized for 1 December 1978 from FGGE analyses and climatological datasets. An adiabatic nonlinear normal mode initialization after Machenauer (1977) is also applied. The model is then integrated forward to the nominal AMIP start date of 1 January 1979.

\section{Time Integration Scheme(s)}

A semi-implicit, semi-Lagrangian time integration scheme with an Asselin (1972) frequency filter is used (cf. Ritchie 1991). Vertical diffusion and surface temperatures and fluxes are computed implicitly (cf. Benoit et al. 1989). The time step is 30 minutes for dynamics and physics, except for full calculations of shortwave and longwave radiative fluxes which are done every 3 hours.

\section{Smoothing / Filling}

Orography is smoothed (see Orography). Negative values of atmospheric specific humidity are temporarily zeroed for use in physical parameterizations, but are not 
permanently filled. The solution in spectral space imposes an approximate conservation of total mass of the atmosphere.

\section{Sampling Frequency}

For the AMIP simulation, the model history is written every 12 hours.

\section{Dynamical/Physical Properties}

\section{Atmospheric Dynamics}

Primitive-equation dynamics expressed in terms of the horizontal vector wind, surface pressure, specific humidity, and temperature are formulated in a semiLagrangian framework (cf. Ritchie 1991).

\section{Diffusion}

- Second-order $\left(\nabla^{2}\right)$ horizontal diffusion is applied to spectral vorticity, divergence, temperature, and specific humidity on constant-sigma surfaces. All diffusivity coefficients are $10^{5} \mathrm{~m}^{2} \mathrm{~s}^{-1}$.

- Vertical diffusion is represented by the turbulence kinetic energy (TKE) closure scheme described by Benoit et al. (1989) and Mailhot and Benoit (1982). Prognostically determined TKE is produced by shear and buoyancy, and is depleted by viscous dissipation. Vertical (but not horizontal) transport of TKE is also modeled, and a minimum background TKE $\left(10^{-4} \mathrm{~m}^{2} \mathrm{~s}^{-2}\right)$ is always present. Diffusion coefficients for momentum and heat/moisture are determined from the current value of TKE and from a locally defined stability-dependent turbulence mixing length. See also Planetary Boundary Layer and Surface Fluxes.

\section{Gravity-wave Drag}

Subgrid-scale parameterization of gravity-wave drag follows the method of McFarlane (1987). Deceleration of resolved flow by breaking/dissipation of orographically excited gravity waves is a function of atmospheric density and the vertical shear of the product of three terms: the Brunt-Vaisalla frequency, the component of local wind in the direction of a near-surface reference level, and a 
displacement amplitude that is bound by the lesser of the subgrid-scale orographic variance (see Orography) or a wave-saturation value.

\section{Solar Constant/Cycles}

The solar constant is the AMIP-prescribed value of $1365 \mathrm{~W} \mathrm{~m}^{-2}$. Both seasonal and diurnal cycles in solar forcing are simulated.

\section{Chemistry}

The carbon dioxide concentration is the AMIP-prescribed value of $345 \mathrm{ppm}$. Monthly climatological zonal profiles of ozone are prescribed after data of Kita and Sumi (1986). Radiative effects of water vapor are also included, but not those of other greenhouse gases or of aerosols (see Radiation).

\section{Radiation}

- The outputs of the shortwave and longwave radiation schemes are the fluxes at each level and the heating rates in each layer. Fluxes also interact with the model at the surface, where the energy balance determines the surface temperature (see Surface Fluxes and Land Surface Processes).

- The shortwave parameterization after Fouquart and Bonnel (1980) considers the effects of carbon dioxide and ozone (see Chemistry), water vapor, clouds, and liquid water. When clouds are present, liquid water is diagnosed from atmospheric temperature: a fraction of the maximum theoretical liquid water concentration on a wet adiabat is assumed, following Betts and Harshvardhan (1987). The entire visible spectrum is treated as a single interval.

- The longwave parameterizations after Garand (1983) and Garand and Mailhot (1990) include the same constituents as in the shortwave scheme, except that liquid water is not interactive. The frequency integration is carried out over 4 spectral intervals: the carbon dioxide 15 micron band divided into center and wing components, the 9.3 micron ozone band, and the rest of the infrared spectrum, including ab-sorption bands for water vapor and continuum absorption. (The frequency integration is precomputed for different temperatures and absorber amounts, with the results stored in look-up tables.) All clouds are assumed to behave as blackbodies (emissivity of 1.0) and to be fully overlapped in the vertical. See also Cloud Formation. 


\section{Convection}

- A modified Kuo (1974) scheme is used to parameterize the effects of deep precipitation-forming convection. When the large-scale vertical motion at the top of the planetary boundary layer (PBL) is upward and the free atmosphere above the PBL top (at about $900 \mathrm{hPa}$ ) is conditionally unstable, the assumed convective activity depends on the net moisture accession in the atmospheric column that is provided by both surface evaporation and large-scale moisture convergence. This moisture is partitioned between a fraction $b$ which moistens the environment, and the remainder $(1-b)$ which contributes to the latent heating (precipitation) rate. Following Anthes (1977), the moistening parameter $b$ is determined as a cubic function of the ratio of the mean relative humidity of the cloud layer to a prescribed critical relative humidity threshold value; if the cloud relative humidity is less than the threshold, $b$ is set to unity (no heating of the environment). The vertical distribution of the heating or moistening is according to differences between mean-cloud and large-scale profiles of temperature and moisture. The mean-cloud profiles are computed from the parcel method slightly modified by an entrainment height of $20 \mathrm{~km}$.

- Shallow convection is parameterized by a generalization of the PBL turbulence formulation (see Diffusion) to include the case of partially saturated air in the conditionally unstable layer above an unstable boundary layer. First, a convective cloud fraction is diagnosed from a relation based on the Bjerknes slice method; then the buoyancy and all the turbulent fluxes are calculated, assuming condensation occurs in that layer fraction. The main effect of the parameterization is to enhance the vertical moisture transport in the absence of large-scale moisture convergence. See also Planetary Boundary Layer.

\section{Cloud Formation}

Convective and stable cloud fractions are diagnosed separately and then combined to interact with the radiative fluxes (see Radiation). In supersaturated absolutely stable layers, a stable cloud fraction of unity is assigned. In layers where shallow or deep convection is diagnosed, the cloud fraction is determined from the pertinent portion of the convective scheme (see Convection). 


\section{RPN}

\section{Precipitation}

Large-scale precipitation forms as a result of condensation in supersaturated layers that are absolutely stable, and shallow convective precipitation in conditionally unstable layers. Deep convective precipitation also forms in association with latent heating in the Kuo (1974) scheme (see Convection). There is subsequent evaporation of large-scale precipitation only.

\section{Planetary Boundary Layer}

The depth of the unstable PBL is determined from the profile of static stability. The depth of the stable PBL is diagnosed using the Monin-Obukhov length. See also Diffusion and Surface Fluxes.

\section{Orography}

The raw topography are from the U.S. Navy data with 10-minutes arc resolution (cf. Josseph 1980) obtained from the European Centre for Medium-Range Weather Forecasts (ECMWF). These heights are spectrally filtered and truncated at the T63 model resolution. The orographic variances required for the gravity-wave drag parameterization (see Gravity-wave Drag) are also determined from the same dataset. Cf. Pellerin and Benoit (1987) for further details.

\section{Ocean}

AMIP monthly sea surface temperature fields are prescribed, with daily values determined by linear interpolation.

\section{Sea Ice}

AMIP monthly sea ice extents are prescribed. The surface temperature of sea ice is predicted by the force-restore method of Deardorff (1978) in the same way as for land points (see Land Surface Processes), without consideration of subsurface heat conduction through the ice. Snow cover is not accounted for on sea ice (see Snow Cover). Cf. Benoit et al. (1989) for further details.

\section{Snow Cover}

Snow mass is not a prognostic variable, and a snow budget is therefore not included. Snow cover over land is prescribed from the monthly climatology of Louis (1984), but 
snow is not specified on sea ice (see Sea Ice). Snow cover alters the albedo (see Surface Characteristics), but not the heat capacity/conductivity of the surface. Sublimation of snow contributes to surface evaporation (see Surface Fluxes), but soil moisture is not affected by snowmelt (see Land Surface Processes). Cf. Benoit et al. (1989) for further details.

\section{Surface Characteristics}

- Over land, surface roughness lengths that are functions of orography and vegetation are specified after Louis (1984). Over sea ice, the prescribed roughness length ranges between $1.5 \times 10^{-5}$ and $5 \times 10^{-3} \mathrm{~m}$. Over ocean, the roughness length is treated as a function of the surface wind stress after the method of Charnock (1955).

- Surface albedos do not depend on solar zenith angle or spectral interval. On land, the surface albedo is specified from annual background values (provided by the Canadian Climate Centre) modulated with the monthly ice (albedo 0.70) and snow (albedo 0.80) climatology (see Snow Cover). The albedo of ocean points is specified to be a uniform 0.07.

- The surface longwave emissivity is prescribed as 0.95 over land and sea ice and as 1.0 (blackbody emission) over ocean.

\section{Surface Fluxes}

- The surface solar absorption is determined from surface albedos, and longwave emission from the Planck equation with prescribed surface emissivities (see Surface Characteristics).

- Following Monin-Obukhov similarity theory, the surface turbulent momentum, sensible heat, and moisture fluxes are expressed as bulk formulae, with drag and transfer coefficients that are functions of surface roughness length (see Surface Characteristics) and of stability (expressed as a bulk Richardson number computed between level sigma $=0.99$ and the surface). The same transfer coefficient is used for the heat and moisture fluxes.

- The flux of surface moisture also depends on an evapotranspiration efficiency factor $\beta$ that is unity over oceans, sea ice, and snow, but that is prescribed as a monthly wetness fraction over land (see Land Surface Processes).

- Above the surface layer, the turbulence closure scheme after Mailhot and Benoit (1982) and Benoit et al. (1989) is used to determine momentum, heat, and moisture fluxes. See also Diffusion and Planetary Boundary Layer. 


\section{RPN}

\section{Land Surface Processes}

- The surface temperature of soil (and of sea ice) is computed by the force-restore method of Deardorff (1978). The upper boundary condition is a net balance of surface energy fluxes (see Surface Fluxes), and monthly deep temperatures are prescribed as a lower boundary condition. The thermodynamic properties are those characteristic of clay soil, and the depth of the soil layer is taken to be that of the penetration of the diurnal heat wave. The same properties are also used for predicting the temperature of sea ice (see Sea Ice).

- Soil moisture (expressed as a wetness fraction) is prescribed from monthly climatologies of Louis (1981). Precipitation and snowmelt therefore do not influence soil moisture, and runoff is not accounted for; however, the prescribed wetness fraction does affect surface evaporation (see Surface Fluxes). Cf. Benoit et al. (1989) for further details. 


\section{SUNYA: State University of New York at Albany}

\section{AMIP Representative(s)}

Dr. Wei-Chyung Wang and Dr. Xin-Zhong Liang, Atmospheric Sciences Research Center, State University of New York at Albany, 100 Fuller Road, Albany, NewYork 12205; Phone: +1-518-442-3816; Fax: +1-518-442-3360; e-mail: wang@climate.asrc.albany.edu (Wang) and liang@climate.asrc.albany.edu (Liang)

\section{Model Designation}

SUNYA CCM1-TG R15L12

\section{Model Lineage}

The SUNYA model is identical to the standard version 1 of the NCAR Community Climate Model (CCM1), except for the addition of radiatively active trace gases other than carbon dioxide. The resulting modified CCM1 is designated as CCM1-TG (see Model Designation).

\section{Model Documentation}

Key documents for the standard CCM1 model are Williamson et al. (1987), Kiehl et al. (1987), and Bath et al. (1987a, b). Wang et al. (1991a, b) describe the treatment of radiative effects of trace gases that are added to CCM1.

\section{Numerical/Computational Properties}

\section{Horizontal Representation}

Spectral (spherical harmonic basis functions) with transformation to a Gaussian grid for calculation of nonlinear quantities and some physics.

\section{Horizontal Resolution}

Spectral rhomboidal 15 (R15), roughly equivalent to $4.5 \times 7.5$ degrees latitudelongitude. 


\section{SUNYA}

\section{Vertical Domain}

Surface to $9 \mathrm{hPa}$; for a surface pressure of $1000 \mathrm{hPa}$, the lowest atmospheric level is at $991 \mathrm{hPa}$.

\section{Vertical Representation \\ Finite-difference sigma coordinates.}

\section{Vertical Resolution}

There are 12 unevenly spaced sigma levels. For a surface pressure of $1000 \mathrm{hPa}$, 3 levels are below $800 \mathrm{hPa}$ and 5 levels are above $200 \mathrm{hPa}$.

\section{Computer/Operating System}

The AMIP simulation was run on a Cray 2 computer using a single processor in a UNICOS environment.

\section{Computational Performance}

For the AMIP experiment, about 1.2 minutes Cray 2 computer time per simulated day.

\section{Initialization}

For the AMIP experiment, initial conditions for the atmospheric state, soil moisture, and snow cover/depth were specified from the NCAR CCM1 model's standard January initial dataset (cf. Bath et al. 1987a). The model then was "spun up" for 210 days in a perpetual January mode. The resulting climate state was then taken as the 1 January 1979 starting point for the AMIP simulation.

\section{Time Integration Scheme(s)}

Time integration is by a semi-implicit Hoskins and Simmons (1975) scheme with an Asselin (1972) frequency filter. The time step is 30 minutes for dynamics and physics, except for full (at all Gaussian grid points and vertical levels) radiation calculations which are done once every 12 hours (see Solar Constant/Cycles). 


\section{Smoothing / Filling}

Orography is smoothed (see Orography). Negative values of atmospheric specific humidity (which arise because of numerical truncation errors in the discretized moisture equation) are filled by horizontal borrowing of moisture in a globally conserving manner. See also Convection.

\section{Sampling Frequency}

For the AMIP simulation, the model history is written once every 12 hours.

\section{Dynamical/Physical Properties}

\section{Atmospheric Dynamics}

Primitive-equation dynamics are expressed in terms of vorticity, divergence, potential temperature, specific humidity, and surface pressure. Energy-conserving vertical finite-difference approximations are utilized (cf. Williamson 1983, 1988).

\section{Diffusion}

- Fourth-order $\left(\nabla^{4}\right)$ horizontal diffusion of vorticity, divergence, temperature, and specific humidity is computed locally on (approximately) constant pressure surfaces in grid-point space, except at stratospheric levels, where second-order $\left(\nabla^{2}\right)$ horizontal diffusion is applied (cf. Boville 1984).

- Stability-dependent vertical diffusion is computed locally in grid-point space at all levels. Cf. Williamson et al. (1987) for further details.

\section{Gravity-wave Drag}

Gravity-wave drag is not modeled.

\section{Solar Constant/Cycles}

The solar constant is the AMIP-prescribed value of $1365 \mathrm{~W} \mathrm{~m}^{-2}$. A seasonal, but not a diurnal cycle, in solar forcing is simulated. 


\section{SUNYA}

\section{Chemistry}

The carbon dioxide concentration is the AMIP-prescribed value of $345 \mathrm{ppm}$. The vertical distribution of zonal-mean mixing ratios of ozone is specified from monthly data of Duetsch (1978), updated by linear interpolation every 12 hours. The radiative effects of water vapor and oxygen, as well as methane, nitrous oxide, and chlorofluorocarbon compounds CFC-11 and CFC-12 also are included, but not those associated with aerosols (see Radiation).

\section{Radiation}

- Shortwave radiation is treated in two spectral intervals--ultraviolet/visible $(0.0$ to 0.9 micron) and near-infrared ( 0.9 to 4.0 microns). Shortwave absorption by ozone, water vapor, carbon dioxide, and oxygen is modeled. Direct-beam absorption by water vapor is after the method of Kratz and Cess (1985); the reflected-beam absorption (as well as Rayleigh scattering by gases) follows Lacis and Hansen (1974). Oxygen absorption is treated as in Kiehl and Yamanouchi (1985), and near-infrared absorption by carbon dioxide is after Sasamori et al. (1972). Gaseous absorption within clouds is included. Cloud albedo depends on optical depth and solar zenith angle, with multiple scattering effects included.

- Longwave radiation is calculated in 5 spectral intervals (with wavenumber boundaries at $0.0,5.0 \times 10^{4}, 8.0 \times 10^{4}, 1.0 \times 10^{5}, 1.2 \times 10^{5}$, and $2.2 \times 10^{5} \mathrm{~m}^{-1}$ ). Absorption/emission by water vapor (cf. Ramanathan and Downey 1986), carbon dioxide (cf. Kiehl and Briegleb 1991), and ozone (cf. Ramanathan and Dickinson 1979) is treated; the standard CCM1 radiation code is modified to include absorption/emission by methane, nitrous oxide, and chlorofluorocarbon compounds CFC-11 and CFC-12 (cf. Wang et al. 1991a, b). The emissivity of nonconvective cloud is a function of diagnostic liquid water content. For purposes of the radiation calculations, cloud is treated as randomly overlapped in the vertical. Cf. Kiehl et al. (1987) and Wang et al. (1991a, b) for further details. See also Cloud Formation.

\section{Convection}

Moist convective adjustment after the method of Manabe et al. (1965) performs several functions: removal of negative atmospheric moisture values (operating with a scheme for horizontal borrowing of moisture--see Smoothing / Filling); dry convective 
adjustment of unsaturated, unstable layers in the model stratosphere, with vertical mixing of moisture; and moist static adjustment of saturated unstable layers and of supersaturated stable layers.

\section{Cloud Formation}

Cloud forms in layers where the relative humidity exceeds 100 percent. If the vertical lapse rate of the layer also exceeds the moist adiabatic value, convective cloud forms (see Convection); otherwise, the cloud is nonconvective, and the fractional cloud cover is set to 0.95 in the layer. Convective cloud cover depends on the depth of the vertical instability, with the cloud amount in each layer adjusted so that the total fractional area is at most 0.30 . If there is no associated precipitation (see Precipitation), a minimum convective cloud fraction of 0.01 is specified in each layer. Cloud is not allowed to form in the lowest model layer or in the top 3 layers, but clouds form together in the second and third layers above the surface if either of these layers is supersaturated. Cf. Kiehl et al. 1987 for further details. See also Radiation for treatment of cloud-radiative interactions.

\section{Precipitation}

Precipitation results from application of convective adjustment (see Convection), if the vertical column is supersaturated with a lapse rate exceeding moist adiabatic. Precipitation also results if the column is supersaturated but with a stable lapse rate. There is no subsequent evaporation of precipitation before it falls to the surface.

\section{Planetary Boundary Layer}

The height of the PBL top is assumed to be that of the first level above the surface (sigma $=0.991$ ), except for the calculation of a bulk Richardson number (see Surface Fluxes). In that case, the PBL top is computed from the temperature at the first sigma level but is constrained to be at least $500 \mathrm{~m}$.

\section{Orography}

After interpolation of $1 \times 1$-degree Scripps Institution surface height data (cf. Gates and Nelson 1975) to the model grid, the data are smoothed using a Gaussian filter with 1.5-degree radius. The resulting heights are transformed into spectral space and truncated at the R15 model resolution. Cf. Pitcher et al. (1983) for further details. 


\section{Ocean}

AMIP monthly sea surface temperature fields are prescribed, with daily values determined by linear interpolation.

\section{Sea Ice}

Monthly AMIP sea ice extents are prescribed. The ice thickness is assumed to be a uniform $2 \mathrm{~m}$, and the sub-ice ocean temperature is specified as a fixed $271.2 \mathrm{~K}$. The surface temperature of the sea ice is computed prognostically by determining heat conduction from the underlying ocean through the ice, following the method of Holloway and Manabe (1971). Sea-ice surface temperature is constrained to be always $<0{ }^{\circ} \mathrm{C}$ (ice melting is not treated), and snow is not allowed to accumulate on the ice (see Snow Cover).

\section{Snow Cover}

Precipitation falls as snow if the temperatures of the surface and the first two atmospheric levels above it are all $<0{ }^{\circ} \mathrm{C}$. Snow cover is determined from a combination of a monthly latitude-dependent climatology (cf. Bath et al. 1987a) and prognostic snow accumulation (on land only) that is determined from a budget equation. A surface temperature $>0{ }^{\circ} \mathrm{C}$ triggers snowmelt, which augments soil moisture (see Land Surface Processes). Snow cover is also depleted by sublimation, which is calculated as part of the surface evaporative flux (see Surface Fluxes).

\section{Surface Characteristics}

- The surface roughness length is specified as a uniform $0.25 \mathrm{~m}$ over land, sea ice, and snow cover, and as $1.0 \times 10^{-3} \mathrm{~m}$ over ocean.

- Surface albedos for land surfaces are derived from the Matthews (1983) $1 \times 1$-degree soil/vegetation dataset, but with distinguished vegetation types reduced to 10 and aggregated to the model resolution (see Horizontal Resolution). Land albedo also depends on solar zenith angle and spectral interval (ultraviolet/ visible vs near-infrared--see Radiation). Snow cover alters the land albedo; the composite value is determined from equally weighted combinations of the local background albedo and that of the snow (which depends on surface temperature for the diffuse beam and on solar zenith angle for the direct beam). Over the ocean, 
surface albedos are prescribed to be 0.0244 for the direct-beam (with sun overhead) and 0.06 for the diffuse-beam component of radiation; the direct-beam albedo varies with solar zenith angle. The albedo of ice is a function of surface temperature. Cf. Briegleb et al. (1986) for further details.

- Longwave emissivities are set to unity (blackbody emission) for all surface types.

Surface Fluxes

- Surface solar absorption is determined from the albedos, and longwave emission from the Planck equation with prescribed surface emissivity of 1.0 (see Surface Characteristics).

- Surface fluxes of momentum, sensible heat and moisture are determined from bulk aerodynamic formulae, following the formulation of Deardorff (1972). Surface drag/exchange coefficients are a function of roughness lengths (see Surface Characteristics) and bulk Richardson number (see Planetary Boundary Layer). For computing these fluxes, the surface wind speed is constrained to be at least $1 \mathrm{~m} \mathrm{~s}^{-1}$.

- The surface moisture flux also depends on the evapotranspiration efficiency $\beta$, which is unity over ocean, snow, and sea ice, but which over land is a function of soil moisture (see Land Surface Processes).

\section{Land Surface Processes}

- Land surface temperature is determined from the balance of surface energy fluxes (see Surface Fluxes) by the diagnostic method of Holloway and Manabe (1971). (That is, there is no heat diffusion/ storage within the soil.)

- Soil moisture is represented by the single-layer "bucket" model of Budyko (1956) and Manabe (1969), with field capacity a uniform $0.15 \mathrm{~m}$ of water. Soil moisture is increased by both precipitation and snowmelt. It is decreased by surface evaporation, which is determined from the product of the evapotranspiration efficiency $\beta$ and the potential evaporation from a surface saturated at the local surface temperature/pressure (see Surface Fluxes). Over land, $\beta$ is given by the ratio of local soil moisture to the field capacity, with runoff occurring implicitly if this ratio exceeds unity. 


\section{SUNYA/NCAR: State University of New York at Albany/ National Center for Atmospheric Research}

\section{AMIP Representative(s)}

Dr. Wei-Chyung Wang, Atmospheric Sciences Research Center, State University of New York at Albany, 100 Fuller Road, Albany, New York 12205; Phone: +1-518-4423357; Fax: +1-518-442-3360; email: wang@climate.asrc.albany.edu

\section{Model Designation}

SUNYA/NCAR GENESIS1.5 T31L18

\section{Model Lineage}

The SUNYA/NCAR model is equivalent to version 1.5 of the GENESIS (Global ENvironmental and Ecological Simulation of Interactive Systems) model, developed by the NCAR Interdisciplinary Climate Systems Section. Version 1.5 stands at an point in development intermediate between its documented predecessor (version 1.02) and a "next-generation" (version 2.0) GENESIS model. (The horizontal/vertical resolution of version 1.5 is enhanced over that of version 1.02 , and some physical parameterizations have also been modified.) The GENESIS atmospheric models are based on the spectral dynamics of the NCAR CCM1 model (cf. Williamson et al. 1987), but their physics schemes differ significantly from those of CCM1.

\section{Model Documentation}

Key documentation for version 1.02 of the GENESIS model (see Model Lineage) is provided by Pollard and Thompson (1992, 1994) and Thompson and Pollard (1994). Changes made in developing version 1.5 will be documented in future papers.

\section{Numerical/Computational Properties}

\section{Horizontal Representation}

Spectral (spherical harmonic basis functions) with transformation to an appropriate nonuniform Gaussian grid for calculation of nonlinear atmospheric quantities. The surface variables (see Ocean, Sea Ice, Snow Cover, Surface Characteristics, 
Surface Fluxes, and Land-Surface Processes) are computed on a uniform latitudelongitude grid of finer resolution (see Horizontal Resolution). Exchanges from the surface to the atmosphere are calculated by area-averaging within the coarser atmospheric Gaussian grid, while bilinear interpolation is used for atmosphere-tosurface exchanges. Atmospheric advection of water vapor (and, on option, other tracers) is via semi-Lagrangian transport (SLT) on the Gaussian grid using cubic interpolation in all directions with operator-splitting between horizontal and vertical advection (cf. Williamson and Rasch 1989 and Rasch and Williamson 1990).

\section{Horizontal Resolution}

The resolution of the model atmosphere is spectral triangular 31 (T31), roughly equivalent to $3.75 \times 3.75$ degrees latitude-longitude. The spectral orography (see Orography) is present at the same resolution, but other surface characteristics and variables are represented on a uniform $2 \times 2$-degree latitude-longitude grid. See also Horizontal Representation.

\section{Vertical Domain}

Surface to $5 \mathrm{hPa}$; for a surface pressure of $1000 \mathrm{hPa}$, the lowest atmospheric level is at $993 \mathrm{hPa}$.

\section{Vertical Representation}

Finite-difference sigma coordinates are used for all atmospheric variables except water vapor, for which hybrid sigma-pressure coordinates (cf. Simmons and Burridge 1981) are employed. Energy-conserving vertical finite-difference approximations are utilized, following Williamson $(1983,1988)$.See also Horizontal Representation and Diffusion.

\section{Vertical Resolution}

There are 18 unevenly spaced sigma (or, for water vapor, hybrid sigma-pressure levels--see Vertical Representation). For a surface pressure of $1000 \mathrm{hPa}, 4$ levels are below $800 \mathrm{hPa}$ and 7 levels are above $200 \mathrm{hPa}$. 


\section{SUNYA/NCAR}

Computer/Operating System

The AMIP simulation was run on a Cray Y/MP computer using a single processor in a UNICOS environment.

\section{Computational Performance}

For the AMIP experiment, about 4 minutes Cray Y/MP computer time per simulated day.

\section{Initialization}

For the AMIP experiment, the model atmosphere, soil moisture, and snow cover/ depth are initialized for 1 January 1979 from the final state of a 200-day reference simulation run in a perpetual January mode. (For the reference simulation, AMIP ocean temperatures/sea ice extents, sun angle, and other calendar-dependent parameters were fixed at 1 January 1979 values, and AMIP solar constant and carbon dioxide concentrations were also used--see Solar Constant/Cycles and Chemistry.)

\section{Time Integration Scheme(s)}

Time integration is by a semi-implicit Hoskins and Simmons (1975) scheme with an Asselin (1972) frequency filter. The time step is 30 minutes for dynamics and physics, except for full radiation calculations. The longwave fluxes are calculated every 30 minutes, but with absorptivities/emissivities updated only once every 24 hours. Shortwave fluxes are computed at 1.5-hour intervals. See also Radiation.

\section{Smoothing / Filling}

Orography is area-averaged (see Orography). Because of the use of the SLT scheme for transport of atmospheric moisture (see Horizontal Representation), spurious negative specific humidity values do not arise, and moisture filling procedures are therefore unnecessary.

\section{Sampling Frequency}

For the AMIP simulation, daily averages of model variables are written once every 24 hours. 


\section{Dynamical/Physical Properties}

\section{Atmospheric Dynamics}

Primitive-equation dynamics are expressed in terms of vorticity, divergence, potential temperature, specific humidity, and the logarithm of surface pressure.

\section{Diffusion}

- In the model troposphere, there is linear biharmonic $\left(\nabla^{4}\right)$ horizontal diffusion of vorticity, divergence, temperature, and specific humidity. In the model stratosphere (top three vertical levels), linear second-order $\left(\nabla^{2}\right)$ diffusion operates, and the diffusivities increase with height. In order to reduce spurious diffusion of moisture in the stratosphere over mountains, the specific humidity is advected on hybrid sigmapressure surfaces, while advection of other fields is on constant sigma surfaces (see Vertical Representation).

- The vertical diffusion of heat, momentum, and moisture is simulated by the explicit modeling of subgrid-scale vertical plumes (see Planetary Boundary Layer and Surface Fluxes).

\section{Gravity-wave Drag}

Orographic gravity-wave drag is parameterized after McFarlane (1987). Deceleration of the resolved flow by dissipation of orographically excited gravity waves is a function of the rate at which the parameterized vertical component of the gravity-wave momentum flux decreases in magnitude with height. This momentumflux term is the product of local air density, the component of the local wind in the direction of that at the near-surface reference level, and a displacement amplitude. At the surface, this amplitude is specified in terms of the subgrid-scale orographic variance, and in the free atmosphere by linear theory, but it is bounded everywhere by wave saturation values. See also Orography.

\section{Solar Constant/Cycles}

The solar constant is the AMIP-prescribed value of $1365 \mathrm{~W} \mathrm{~m}^{-2}$. Both seasonal and diurnal cycles in solar forcing are simulated. 


\section{Chemistry}

The carbon dioxide concentration is the AMIP-prescribed value of $345 \mathrm{ppm}$. Monthly global ozone concentrations are as described by Wang et al. (1994). (Total column ozone is taken from data of Bowman and Krueger 1985 and Stolarski et al. 1991. The stratospheric distribution up to $60 \mathrm{~km}$ is based on data of Cunnold et al. 1989 and McCormick et al. 1992; above $60 \mathrm{~km}$, a single mean value at $100 \mathrm{~km}$ taken from McClatchey et al. 1971 is used to calculate ozone mixing ratios. Tropospheric ozone is specified from data of Logan 1985 and Spivakovsky et al. 1990.) Radiative effects of oxygen, water vapor, methane, nitrous oxide, chlorofluorocarbon compounds CFC-11 and CFC-12, and of preindustrial tropospheric "background" aerosol are also included (see Radiation).

\section{Radiation}

- Shortwave radiation is treated by a modified Thompson et al. (1987) scheme in ultraviolet/visible (0.0 to $0.90 \mathrm{micron}$ ) and near-infrared (0.90 to $4.0 \mathrm{microns})$ spectral bands. Gaseous absorption is calculated from broadband formulas of Ramanathan et al. (1983), with ultraviolet/visible absorption by ozone and near-infrared absorption by water vapor, oxygen, and carbon dioxide treated. Reflectivities from multiple Rayleigh scattering are determined from a polynomial fit in terms of the gaseous optical depth and the solar zenith angle. A delta-Eddington approximation is used to calculate shortwave albedos and transmissivities of aerosol (see Chemistry) and of cloudy portions of each layer. Cloud optical properties depend on liquid water content (LWC), which is prescribed as a function of height (cf. Slingo and Slingo 1991). Clouds that form in individual layers (see Cloud Formation) are assumed to be randomly overlapped in the vertical. The effective cloud fraction depends on solar zenith angle (cf. Henderson-Sellers and McGuffie 1990) to allow for the three-dimensional blocking effect of clouds at low sun angles.

- Longwave radiation is calculated in 5 spectral intervals (with wavenumber boundaries at $0.0,5.0 \times 10^{4}, 8.0 \times 10^{4}, 1.0 \times 10^{5}, 1.2 \times 10^{5}$, and $2.2 \times 10^{5} \mathrm{~m}^{-1}$ ). Broadband absorption and emission by water vapor (cf. Ramanathan and Downey 1986), carbon dioxide (cf. Kiehl and Briegleb 1991), and ozone (cf. Ramanathan and Dickinson 1979) are included. In addition, there is explicit treatment of individual greenhouse trace gases (methane, nitrous oxide, and chlorofluorocarbon compounds 
CFC-11 and CFC-12: cf. Wang et al. 1991a,b). Cloud emissivity depends on prescribed LWC (see above). See also Cloud Formation.

\section{Convection}

Dry and moist convection as well as vertical mixing in the planetary boundary layer (PBL) are treated by an explicit model of subgrid-scale vertical plumes following the approach of Kreitzberg and Perkey (1976) and Anthes (1977), but with simplifications. A plume may originate from any layer, and accelerate upward if buoyantly unstable; the plume radius and fractional coverage of a grid box are prescribed as a function of height. Mixing with the large-scale environmental air (entrainment and detrainment) is proportional to the plume vertical velocity. From solution of the subgrid-scale plume model for each vertical column, the implied grid-scale vertical fluxes, latent heating, and precipitation are deduced. Convective precipitation forms if the plume air is supersaturated; its subsequent evaporation in falling toward the surface (see Precipitation) substitutes for explicit treatment of convective downdrafts and cloud/precipitation microphysics. See also Planetary Boundary Layer.

\section{Cloud Formation}

- Cloud formation follows a modified Slingo and Slingo (1991) scheme that accounts for convective, anvil cirrus, and stratiform cloud types. In a vertical column, the depth of convective cloud is determined by the vertical extent of buoyant plumes (see Convection), and the cloud fraction from a function of the instantaneous convective precipitation rate. (The convective cloud fraction is adjusted in accord with the assumption of random vertical overlap of cloud--see Radiation). If the convective cloud penetrates higher than a sigma level of about 0.6 , anvil cirrus also forms.

- The fraction of stratiform (layer) cloud is a function of the relative humidity excess above a threshold that depends on sigma level. In order to predict realistic amounts of stratus cloud in winter polar regions, a further constraint on cloud formation in conditions of low absolute humidity is added, following Curry and Herman (1985).

\section{Precipitation}

- Precipitation forms in association with subgrid-scale supersaturated convective plumes (see Convection). Under stable conditions, precipitation also forms to restore the large-scale supersaturated humidity to its saturated value. Both convective and 


\section{SUNYANCAR}

large-scale precipitation evaporate in falling toward the surface. The amount of evaporation is parameterized as a function of the large-scale humidity and the thickness of the intervening atmospheric layers.

- In addition, subgrid-scale spatial variability of convective precipitation falling in land grid boxes is simulated stochastically (cf. Thomas and Henderson-Sellers 1991). See also Snow Cover and Land Surface Processes.

\section{Planetary Boundary Layer}

Vertical mixing in the PBL (and above the PBL for an unstable vertical lapse rate) is simulated by an explicit model of subgrid-scale plumes (see Convection) that are initiated at the center of the lowest model layer using scaled perturbation quantities from the constant-flux region immediately below (see Surface Fluxes). The plume vertical motion and perturbation temperature, specific humidity, and horizontal velocity components are solved as a function of height. The implied grid-scale fluxes are then used to modify the corresponding mean quantities.

\section{Orography}

Raw orography obtained from the $1 \times 1$-degree topographic height data of Gates and Nelson (1975) is area-averaged over each atmospheric grid box (see Horizontal Representation). The subgrid-scale orographic variances required by the gravity-wave drag parameterization (see Gravity-wave Drag) are obtained from U.S. Navy data with resolution of 10 minutes arc (cf. Joseph 1980). The standard deviation (square root of the variance) of the fine-scale U.S. Navy orography in each model grid box is computed, and 75 percent of that value is added to the basic Gates-Nelson orographic height. The resulting "envelope orography" is transformed to spectral space and truncated at the T31 atmospheric model resolution (see Horizontal Resolution).

\section{Ocean}

AMIP monthly sea surface temperature fields are prescribed, with daily values determined by linear interpolation.

Sea Ice

Monthly AMIP sea ice extents are prescribed, with fractional coverage of a $2 \times 2$-degree grid box allowed. A six-layer model (top layer a constant $0.03 \mathrm{~m}$ thick, 
other layers of equal thickness at each time step) similar to that of Semtner (1976) is used to simulate linear heat diffusion through the ice. Prognostic variables include the layer temperatures, the total ice thickness, and brine-reservoir heating. The temperature of any layer exceeding the ice melting point $\left(0^{\circ} \mathrm{C}\right)$ is reset to melting, and the excess heat is given to a brine reservoir (with capacity 25 percent of the heat required to melt the entire ice column at the current time step), or is used to melt part of the ice column if the reservoir is full. The upper boundary condition is the net balance of surface energy fluxes (see Surface Fluxes); the bottom ice surface remains at the ocean freezing temperature $(271.2 \mathrm{~K})$. Snow accumulates on sea ice (see Snow Cover) and may augment the ice thickness: snow is converted instantaneously to ice if the snow depth is enough to hydrostatically depress the snow-ice interface below the ocean surface. See also Surface Characteristics.

\section{Snow Cover}

- Precipitation falls as snow if the surface air temperature is $<0^{\circ} \mathrm{C}$, with accumulation on land and continental/sea ice surfaces. Snow cover is simulated by a three-layer model (top layer a constant $0.03 \mathrm{~m}$ thick, other layers of equal thickness at each time step). Prognostic variables include the layer temperatures and the total snow mass per unit horizontal area (expressed as snow thickness and fractional coverage in a $2 \times 2$-degree grid box). When snow falls in a previously snow-free grid box, the fractional coverage increases from zero, with total snow thickness fixed at $0.15 \mathrm{~m}$. If $\mathrm{f}$ snowfall continues, the fractional coverage increases up to 100 percent, after which the snow thickness increases (the reverse sequence applies for melting of a thick snow cover).

- Heat diffuses linearly with temperature within and below the snow. The upper boundary condition is the net balance of surface energy fluxes, and the lower condition is the net heat flux at the snow-surface interface (see below). If the temperature of any snow layer becomes $>0{ }^{\circ} \mathrm{C}$, it is reset to $0{ }^{\circ} \mathrm{C}$, snow is melted to conserve heat, and the meltwater contributes to soil moisture. Snow cover is also depleted by sublimation (a part of surface evaporation--see Surface Fluxes), and snow modifies the roughness and the albedo of the surface (see Surface Characteristics).

- The fractional coverage of snow is the same for both bare ground and lowerlayer vegetation (see Land Surface Processes). In order to exactly conserve heat, temperatures are kept separately for buried and unburied lower-layer vegetation, and are adjusted calorimetrically as the snow cover grows/recedes. Any liquid water or snow 
already intercepted by the vegetation canopy that becomes buried is immediately incorporated into the lowest snow layer. The buried lower vegetation is included in the vertical heat diffusion equation as an additional layer between the soil and the snow, with thermal conduction depending on the local vegetation fractional coverage and leaf/stem area indices. See also Sea Ice.

\section{Surface Characteristics}

- The land surface is subdivided according to upper- and lower-story vegetation (trees and grass/shrubs) of 12 types. Vegetation attributes (e.g., fractional cover and heights, leaf and stem area indices, leaf orientation, root distribution, leaf/stem optical properties, and stomatal resistances) are specified from a detailed equilibrium vegetation model driven by present-day climate. Soil hydraulic properties are inferred from texture data of Webb et al. (1993) that consider 15 soil horizons, 107 soil types, and 10 continental subtypes. See also Land Surface Processes.

- The surface roughness length is a uniform $1.0 \times 10^{-4} \mathrm{~m}$ over the oceans and $5.0 \times 10^{-4} \mathrm{~m}$ over ice and snow surfaces. The spatially variable roughness over vegetated land is calculated as described by Pollard and Thompson (1994); the roughness of all bare-soil areas is $5 \times 10^{-3} \mathrm{~m}$.

- The ocean surface albedo is specified after Briegleb et al. (1986) to be 0.0244 for the direct-beam component of radiation (with sun overhead), and a constant 0.06 for the diffuse-beam component; the direct-beam albedo varies with solar zenith angle, but not spectral interval. The albedo of ice surfaces depends on the topmost layer temperature (to account for the lower albedo of melt ponds). For temperatures that are $<-5{ }^{\circ} \mathrm{C}$, the ice albedos for the ultraviolet/visible and near-infrared spectral bands (see Radiation) are 0.8 and 0.5 respectively; these decrease linearly to 0.7 and 0.4 as the temperature increases to $0^{\circ} \mathrm{C}$ (cf. Harvey 1988). There is no dependence on solar zenith angle or direct-beam vs diffuse-beam radiation. Following Maykut and Untersteiner (1971), a fraction 0.17 of the absorbed solar flux penetrates and warms the ice to an e-folding depth of $0.66 \mathrm{~m}$ (see Sea Ice). Over vegetated land, instantaneously changing (depending on solar zenith angle) spatially varying albedos are calculated as described by Pollard and Thompson (1994) for direct and diffuse radiation in visible (0.4-0.7 micron) and near-infrared (0.7-4.0 microns) spectral intervals. Albedos of bare dry soil are prescribed as a function of spectral interval and the texture of the topmost soil layer (cf. Webb et al. 1993); these values are modified 
by the moisture in the top soil layer (see Land Surface Processes), but they do not depend on solar zenith angle or direct-beam vs diffuse beam radiation. The background albedos of land and ice surfaces are also modified by snow (see Snow Cover). The snow albedo depends on the temperature (wetness) of the topmost snow layer: below $-15^{\circ} \mathrm{C}$, the visible and near-infrared albedos are 0.9 and 0.6 , respectively; these decrease linearly to 0.8 and 0.5 as the temperature increases to $0^{\circ} \mathrm{C}$ (cf. Harvey 1988). The direct-beam snow albedo also depends on solar zenith angle (cf. Briegleb and Ramanathan 1982).

- Longwave emissivities of ocean and ice surfaces are unity (blackbody emission), but over land they are a function of vegetation (the emissivity of each canopy layer depends on leaf/stem densities).

\section{Surface Fluxes}

- Surface solar absorption is determined from albedos, and longwave emission from the Planck equation with prescribed surface emissivities (see Surface Characteristics).

- Turbulent vertical eddy fluxes of momentum, heat, and moisture are expressed as bulk formulae, following Monin-Obukhov similarity theory. The values of wind, temperature, and humidity required for the bulk formulae are taken to be those at the lowest atmospheric level (sigma $=0.993$ ), which is assumed to be within a constant-flux surface layer. The bulk drag/transfer coefficients are functions of roughness length (see Surface Characteristics) and stability (bulk Richardson number), following the method of Louis et al. (1981). Over vegetation, the turbulent fluxes are mediated by a Land-Surface-Transfer (LSX) model (see Land Surface Processes). The bulk formula for the surface moisture flux also depends on the surface specific humidity, which is taken as the saturated value over ocean, snow, and ice surfaces, but which otherwise is a function of soil moisture.

- Above the surface layer, the turbulent diffusion of momentum, heat, and moisture is simulated by a subgrid-scale plume model (see Planetary Boundary Layer).

\section{Land Surface Processes}

- Effects of interactive vegetation are simulated by the LSX model (cf. Pollard and Thompson 1994, Thompson and Pollard 1994), which includes canopies in upper (trees) and lower (grasses/shrubs) layers. Prognostic variables are the temperatures 


\section{SUNYA/NCAR}

of upper-layer leaves and stems and of combined lower-layer leaves/stems, as well as the stochastically varying rain and snow intercepted by these three components (see Precipitation and Snow Cover). The LSX model also includes evaporation of canopyintercepted moisture and evapotranspiration via root uptake, as well as soil wilting points. Air temperatures/specific humidities within the canopies are determined from the atmospheric model and the surface conditions; canopy aerodynamics are modeled using logarithmic wind profiles above/between the vegetation layers, and a simple diffusive model of air motion within each layer. Effects of vegetation patchiness on radiation and precipitation interception are also included.

- Soil temperature and fractional liquid water content are predicted in 6 layers with thicknesses $0.05,0.10,0.20,0.40,1.0$, and 2.5 meters, proceeding downward. (The near-surface temperature profile of the continental ice sheets is predicted by the same model.) Heat diffuses linearly, but diffusion/drainage of liquid water is a nonlinear function of soil moisture (cf. Clapp and Hornberger 1978). Boundary conditions at the bottom soil level include zero diffusion of heat and liquid, but nonzero gravitational drainage (deep runoff). The upper boundary condition for heat is the net energy flux at the soil surface computed by the LSX model; infiltration of moisture is limited by the downward soil diffusion to the center of the upper layer, assuming a saturated surface (cf. Abramopoulos et al. 1988). A ponding reservoir (of few-centimeter capacity) simulates a "retention layer" that delays surface runoff, which occurs when the moisture capacity of the topmost soil layer is exceeded.

- Soil fractional ice content is also predicted. (Ice formation affects soil hydraulics by impeding water flow, and soil thermodynamics by changing the heat capacity/ conductivity and by releasing latent heat.) The specific humidity at the upper surface of the top soil layer (used to predict evaporation--see Surface Fluxes) varies as the square of the composite liquid/ice fractions. See also Snow Cover and Surface Characteristics. 


\section{UCLA: University of California at Los Angeles}

\section{AMIP Representative(s)}

Dr. Carlos R. Mechoso, Department of Atmospheric Sciences, University of California, 405 Hilgard Avenue, Los Angeles, California, 90024-1565; Phone: +1-310206-5253; Fax: +1-310-206-5219; e-mail: mechoso@atmos.ucla.edu

\section{Model Designation}

UCLA AGCM6.4 4x 5L15

\section{Model Lineage}

The UCLA model derives from an earlier version described by Arakawa and Lamb (1977). Subsequent modifications mainly include changes in finite-difference schemes and in the parameterizations of radiation, convection, and planetary boundary layer $(\mathrm{PBL})$.

\section{Model Documentation}

Key documentation of model features is provided by Arakawa (1972), Arakawa and Lamb (1977, 1981), Arakawa and Schubert (1974), Arakawa and Suarez (1983), Lord (1978), Lord and Arakawa (1980), Lord et al. (1982), Randall et al. (1985), Suarez et al. (1983), and Takano and Wurtele (1982).

\section{Numerical/Computational Properties}

\section{Horizontal Representation}

Finite differences on a staggered latitude-longitude C-grid (cf. Arakawa and Lamb 1977). The horizontal advection of momentum is treated by the potentialenstrophy conserving scheme of Arakawa and Lamb (1981), modified to give fourthorder accuracy for the advection of potential vorticity (cf. Takano and Wurtele 1981). The horizontal advection scheme is also fourth-order for potential temperature (conserving the global mass integral of its square), and for water vapor and prognostic ozone (see Chemistry). The differencing of the continuity equation and the pressure gradient force is of second-order accuracy. 


\section{UCLA}

\section{Horizontal Resolution}

$4 \times 5$-degree latitude-longitude grid.

\section{Vertical Domain}

Surface to $1 \mathrm{hPa}$. The lowest atmospheric layer is identically the planetary boundary layer (PBL), whose depth is a prognostic variable. See also Vertical Representation, Vertical Resolution, and Planetary Boundary Layer.

\section{Vertical Representation}

- Finite differences in modified sigma coordinates. For $\mathrm{P}$ the pressure at a given level, $\mathrm{P}_{\mathrm{T}}=1 \mathrm{hPa}$ the constant pressure at the model top, $\mathrm{P}_{\mathrm{I}}=100 \mathrm{hPa}$ the pressure at a level near the tropopause, $\mathrm{P}_{\mathrm{B}}$ the pressure at the top of the planetary boundary layer, and $P_{S}$ the pressure at the earth's surface, sigma $=\left(P-P_{I}\right) /\left(P_{I}-P_{T}\right)$ for $P_{I} \geq P \geq P_{T}$ (in the stratosphere); sigma $=\left(P-P_{I}\right) /\left(P_{B}-P_{I}\right)$ for $P_{B} \geq P \geq P_{I}$ (in the troposphere above the $\mathrm{PBL}$ ); and sigma $=1+\left(\mathrm{P}-\mathrm{P}_{\mathrm{B}}\right) /\left(\mathrm{P}_{\mathrm{S}}-\mathrm{P}_{\mathrm{B}}\right)$ for $\mathrm{P}_{\mathrm{S}} \geq \mathrm{P} \geq \mathrm{P}_{\mathrm{B}}$ (in the $\mathrm{PBL}$ ). The sigma levels above $100 \mathrm{hPa}$ are evenly spaced in the logarithm of pressure (cf. Suarez et al. 1983).

- The vertical differencing scheme after Arakawa and Suarez (1983) and Tokioka (1978) conserves global mass integrals of potential temperature and total potential plus kinetic energy for frictionless adiabatic flow. See also Vertical Resolution and Planetary Boundary Layer.

\section{Vertical Resolution}

There are 15 levels in modified sigma coordinates (see Vertical Representation). The first level above the surface is identically the prognostic PBL top (see Planetary Boundary Layer). For a surface pressure of $1000 \mathrm{hPa}, 2$ levels are typically below $800 \mathrm{hPa}$ (depending on PBL depth) and 9 levels are above $200 \mathrm{hPa}$.

\section{Computer/Operating System}

The AMIP simulation was run on a Cray C90 computer using a single processor in the UNICOS environment. 


\section{Computational Performance}

For the AMIP experiment, about 1.5 minutes of Cray C90 computer time per simulated day.

\section{Initialization}

For the AMIP simulation, the model atmosphere and snow cover/depth are initialized for 1 January 1979 from FGGE III-B data. Soil moisture is initialized from the January climatological estimates of Mintz and Serafini (1981).

\section{Time Integration Scheme(s)}

The model is integrated by the leapfrog scheme at time steps of 7.5 minutes, with a Matsuno step inserted hourly. At the forward stage of the Matsuno step, all diabatic and dissipative terms (including radiative fluxes), sources and sinks in atmospheric water vapor and prognostic ozone (see Chemistry), and the depth of the PBL (see Planetary Boundary Layer) are recalculated.

\section{Smoothing / Filling}

Orography is area-averaged (see Orography). A specially constructed Fourier filter damps out numerically unstable modes (cf. Arakawa and Lamb 1977). The prognostic PBL depth (see Planetary Boundary Layer) is also smoothed. Negative values of ozone and atmospheric moisture are avoided by suitable vertical interpolation at half-levels and by modification of the horizontal differencing scheme to prevent advection from grid boxes with zero or negative concentrations (cf. Arakawa and Lamb 1977).

\section{Sampling Frequency}

For the AMIP simulation, the model history is written every 6 hours.

\section{Dynamical/Physical Properties}

\section{Atmospheric Dynamics}

Primitive-equation dynamics are expressed in terms of $u$ and $v$ winds, potential temperature, specific humidity, and surface pressure. The concentration of ozone and 


\section{UCLA}

the depth of the PBL are also prognostic variables (see Chemistry and Planetary Boundary Layer ).

\section{Diffusion}

- Nonlinear second-order horizontal diffusion after Smagorinsky (1963) is applied (with a small coefficient) only to the momentum equation on the modified sigma levels (see Vertical Representation).

- Vertical diffusion is not explicitly included; however, momentum is redistributed by cumulus convection (see Convection).

\section{Gravity-wave Drag}

Drag associated with orographic gravity waves is simulated after the method of Palmer et al. (1985), using directionally dependent subgrid-scale orographic variances obtained from the U.S. Navy dataset (cf. Joseph 1980).

\section{Solar Constant/Cycles}

The solar constant is the AMIP-prescribed value of $1365 \mathrm{~W} \mathrm{~m}^{-2}$. Both seasonal and diurnal cycles in solar forcing are simulated.

\section{Chemistry}

The carbon dioxide concentration is the AMIP-prescribed value of $345 \mathrm{ppm}$. Ozone is a prognostic variable, with its photochemistry parameterized following Schlesinger (1976) and Schlesinger and Mintz (1979). The radiative effects of water vapor are also treated, but not those of aerosols (see Radiation).

\section{Radiation}

Shortwave radiation is parameterized after Katayama (1972) with modifications by Schlesinger (1976). Following Joseph (1970), the radiation is divided into absorbed and scattered components. Absorption by water vapor and ozone is modeled using wavelength-integrated transmission functions of Yamamoto (1962) and Elsasser (1960). Rayleigh scattering is interpolated from calculations of Coulson (1959). Absorption and scattering by aerosols are not included. The radiatively active low, middle, and high clouds (see Cloud Formation) are assigned different absorptivities and reflectivities per 
unit thickness. Cloud albedo and absorptivity are functions of cloud thickness, height, solid and liquid water content, water vapor content, and solar zenith angle, following Rodgers (1967a). Multiple scattering effects of clouds are treated as in Lacis and Hansen (1974).

- The parameterization of longwave radiation follows the approach of Harshvardhan et al. (1987). For absorption calculations, the broadband transmission method of Chou (1984) is used for water vapor, that of Chou and Peng (1983) for carbon dioxide, and that of Rodgers (1968) for ozone. Longwave absorption is calculated in five spectral bands (with wavenumbers between 0 to $3 \times 10^{5} \mathrm{~m}^{-1}$ ), with continuum absorption by water vapor following Roberts et al. (1976). Cloud longwave emissivities are treated as in Harshvardhan et al. (1987). Clouds are assumed to be fully overlapped in the vertical (radiatively active clouds fill the grid box). See also Cloud Formation.

\section{Convection}

- Simulation of cumulus convection (with momentum transport) is based on the scheme of Arakawa and Schubert (1974), as implemented by Lord (1978), Lord and Arakawa (1980), and Lord et al. (1982). Mass fluxes are predicted from mutually interacting cumulus subensembles which have different entrainment rates and levels of neutral buoyancy that define the tops of the clouds and their associated convective updrafts. In turn, these mass fluxes feed back on the large-scale fields of temperature, moisture, and momentum (through cumulus friction). The effects of phase changes from water to ice on convective cloud buoyancy are also accounted for, but those of convective-scale downdrafts are not explicitly simulated.

- The mass flux for each cumulus subensemble is predicted from an integral equation that includes a positive-definite work function (defined by the tendency of cumulus kinetic energy for the subensemble) and a negative-definite kernel which expresses the effects of other subensembles on this work function. The predicted mass fluxes are positive-definite optimal solutions of this integral equation under a quasiequilibrium constraint (cf. Lord et al. 1982).

- A moist convective adjustment process simulates midlevel convection that originates above the PBL. When the lapse rate exceeds moist adiabatic from one layer to the next and saturation occurs in both layers, mass is mixed such that either the lapse 


\section{UCLA}

rate is restored to moist adiabatic or saturation is eliminated. Any resulting supersaturation is removed by formation of large-scale precipitation (see Precipitation). In addition, if the lapse rate becomes dry convectively unstable anywhere within the model atmosphere, moisture and enthalpy are redistributed vertically, effectively deepening the PBL (see Planetary Boundary Layer). Cf. Suarez et al. (1983) for further details.

\section{Cloud Formation}

- Four types of cloud are simulated: penetrative cumulus, midlevel convective, PBL stratus, and large-scale condensation cloud. Of these, the PBL, large-scale, and cumulus clouds above $400 \mathrm{hPa}$ interact radiatively (see Radiation); these are assumed to fill the grid box completely (cloud fraction of 1).

- Large-scale condensation cloud forms in layers that are saturated. The penetrative cumulus cloud and midlevel convective cloud are associated, respectively, with the cumulus convection and moist adjustment schemes (see Convection). PBL stratus cloud forms if the specific humidity at the PBL top is greater than saturation, and the cloud-top stability criterion of Randall (1980) is met. The base of this cloud is determined as the level at which the specific humidity of the well-mixed PBL is equal to the saturation value (see Planetary Boundary Layer).

\section{Precipitation}

Precipitation may occur above the PBL from cumulus convection and from moist convective adjustment (see Convection). Precipitation also results from large-scale supersaturation of a vertical layer. Subsequent evaporation of falling precipitation is not treated.

\section{Planetary Boundary Layer}

- The PBL is parameterized as a well-mixed layer (turbulent fluxes linear in the vertical) whose depth varies as a function of horizontal mass convergence, entrainment, and cumulus mass flux determined from the convective parameterization (see Convection). The PBL is identical to the lowest model layer, and its potential temperature, $u-v$ winds, and specific humidity are prognostic variables. Turbulence kinetic energy (TKE) due to shear production is also determined by a closure condition involving dissipation, buoyant consumption, and the rate at which TKE is supplied to 
make newly entrained air turbulent. Because of the absence of vertical diffusion above the PBL (see Diffusion), discontinuities in atmospheric variables that may exist at the PBL top are determined from "jump" equations.

- The presence of PBL stratocumulus cloud affects the radiative parameterizations (see Cloud Formation and Radiation), the entrainment rate (through enhanced cloud-top radiative cooling and latent heating), and the exchange of mass with the layer above the PBL as a result of layer cloud instability. If the PBL lapse rate is dry convectively unstable, an adjustment process restores stability by redistributing moisture and enthalpy vertically (see Convection). Cf. Suarez et al. (1983) and Randall et al. (1985) for further details. See also Surface Characteristics and Surface Fluxes.

\section{Orography}

Raw orography is obtained from a U.S. Navy dataset (cf. Joseph 1980) with resolution of 10 minutes arc on a latitude-longitude grid. These terrain heights are areaaveraged on the $4 \times 5$-degree model grid, and the subgrid-scale orographic variances about these means are also computed (see Gravity-wave Drag).

\section{Ocean}

AMIP monthly sea surface temperature fields are prescribed, with daily values determined by linear interpolation.

\section{Sea Ice}

AMIP monthly sea ice extents are prescribed, with daily values determined from linear interpolation. The daily thickness of sea ice varies linearly between 0 and 3 meters in the first and last month in which it is present; otherwise, the thickness remains a constant 3 meters. The surface temperature of sea ice is determined from an energy balance that includes the surface heat fluxes (see Surface Fluxes) as well as the heat conducted through the ice from the ocean below (at a fixed temperature). Snow is not allowed to accumulate on sea ice, nor to modify its albedo or thermodynamic properties.

\section{Snow Cover}

Precipitation falls as snow if the surface air temperature is $<273.1 \mathrm{~K}$. Snow accumulates only on land, covering each grid box completely. Snow cover affects the 


\section{UCLA}

land surface albedo, but not its thermal properties. Snow mass is a prognostic variable, but sublimation is not included in the snow budget equation. Snowmelt affects the ground temperature, but not soil moisture. See also Surface Characteristics and Land Surface Processes.

\section{Surface Characteristics}

- The surface roughness lengths are specified as uniform values of $2.0 \times 10^{-4} \mathrm{~m}$ over ocean, $1 \times 10^{-4} \mathrm{~m}$ over sea ice, and $1 \times 10^{-2} \mathrm{~m}$ over continental ice. The roughness lengths over land vary monthly according to 12 vegetation types (cf. Dorman and Sellers 1989), with daily values determined by linear interpolation.

- The snow-free land albedo varies monthly according to vegetation type, with daily values determined by linear interpolation. Albedos of ocean, ice, and snowcovered surfaces are prescribed and do not depend on solar zenith angle or spectral interval.

- Longwave emissivity is prescribed as unity (blackbody emission) for all surfaces.

\section{Surface Fluxes}

- Surface solar absorption is determined from the albedos, and longwave emission from the Planck equation with prescribed emissivity of 1.0 (see Surface Characteristics).

- Turbulent eddy fluxes of momentum, heat, and moisture are parameterized as bulk formulae with drag/transfer coefficients that depend on vertical stability (bulk Richardson number) and the locally variable depth of the PBL normalized by the surface roughness length (see Surface Characteristics), following Deardorff (1972). The requisite surface atmospheric values of wind, dry static energy, and humidity are taken to be the bulk values of these variables predicted in the PBL (see Planetary Boundary Layer). The same exchange coefficient is used for the surface moisture flux as for the sensible heat flux.

- The surface moisture flux also depends on an evapotranspiration efficiency factor $\beta$, which is set to unity over ocean and ice surfaces, but which is equal to a prescribed soil wetness fraction (see Land Surface Processes) that depends on vegetation type (cf. Dorman and Sellers 1989). 


\section{Land Surface Processes}

- Ground temperature is determined from a surface energy balance (see Surface Fluxes) without inclusion of soil heat storage (cf. Arakawa 1972).

- Soil moisture (expressed as a wetness fraction) is prescribed monthly from climatological estimates (of Mintz and Serafini 1981). Precipitation and snowmelt therefore do not influence soil moisture, and runoff is not accounted for; however, the prescribed soil moisture does affect surface evaporation (see Surface Fluxes). 


\section{UGAMP: The UK Universities' Global Atmospheric Modelling Programme}

\section{AMIP Representative(s)}

Dr. Mike Blackburn and Dr. Julia Slingo, Department of Meteorology, University of Reading, 2 Earley Gate, Whiteknights, PO Box 239, Reading RG6 2AU, England; Phone: +44-734-318327 (Blackburn) and +44-734-318424 (Slingo); Fax: +44-734318316; e-mail: mb2@ib.rl.ac.uk (Blackburn) and swssling@swssner1.rdg.ac.uk (Slingo)

\section{Model Designation \\ UGAMP UGCM1.3 T42L19}

\section{Model Lineage}

The UGAMP model is based on the ECMWF(cycle 27) model (cf. Tiedtke et al. 1988 and Simmons et al. 1989), but with modifications principally in the treatment of radiation, convection, surface fluxes, vertical advection, and lateral and vertical dissipation.

\section{Model Documentation}

Documentation for the ECMWF(cycle 27) predecessor model is provided by Tiedtke et al. (1988). Subsequent modifications are described by Slingo et al. (1994) and references therein.

\section{Numerical/Computational Properties}

\section{Horizontal Representation}

Spectral (spherical harmonic basis functions) with transformation to a Gaussian grid for calculation of nonlinear quantities and some physics.

\section{Horizontal Resolution}

Spectral triangular 42 (T42), roughly equivalent to $2.8 \times 2.8$ degrees latitudelongitude. The transform grid is sufficient to prevent aliasing of quadratic quantities, with 128 equispaced longitudes and 64 Gaussian latitudes. The full radiative calculations are performed on a reduced longitudinal grid, retaining only the first 16 Fourier modes (see Radiation). 


\section{Vertical Domain}

Surface to $10 \mathrm{hPa}$; for a surface pressure of $1000 \mathrm{hPa}$, the lowest atmospheric level is at about $996 \mathrm{hPa}$.

\section{Vertical Representation}

Hybrid sigma-pressure coordinates after Simmons and Burridge (1981) and Simmons and Struefing (1981). To avoid oscillations in the profile of an advected quantity with rapidly changing gradient, vertical advection is treated by the Total Variation Diminishing (TVD) scheme of Thuburn (1993).

\section{Vertical Resolution}

There are 19 irregularly spaced hybrid levels. For a surface pressure of $1000 \mathrm{hPa}$, 5 levels are below $800 \mathrm{hPa}$ and 7 levels are above $200 \mathrm{hPa}$.

\section{Computer/Operating System}

The AMIP simulation was run on a Cray 2 computer using a single processor in a UNICOS environment.

\section{Computational Performance}

For the AMIP experiment, about 8 minutes Cray 2 computer time per simulated day (including data-archiving and storage time).

\section{Initialization}

For the AMIP experiment, the model atmosphere, soil moisture, and snow cover/ depth were initialized from the ECMWF operational analysis for $12 \mathrm{Z}$ on 15 January 1987. These initial conditions were then designated as for $12 Z$ on 15 December 1978 , and the model was (partially) "spun up" to the AMIP start time by integrating it to a simulated state for $00 Z$ on 1 January 1979 with prescribed (and fixed) AMIP sea surface temperatures for January 1979. See also Ocean.

\section{Time Integration Scheme(s)}

The time integration is by a semi-implicit Hoskins and Simmons (1975) scheme with an Asselin (1972) time filter. Advection of vorticity and moisture by a zonally 
symmetric flow is also treated implicitly. The time step is 30 minutes for dynamics and physics, except for full radiation/cloud calculations once every 3 hours (on a reduced grid, at every fourth point in longitude only--see Radiation). To ensure mass conservation, the global mean value of the logarithm of surface pressure is rescaled at each time step (but with mass sources/sinks associated with evaporation/precipitation neglected).

\section{Smoothing / Filling}

Orography is smoothed (see Orography). Negative values of atmospheric specific humidity (due to numerical truncation errors in the discretized moisture equation) are filled by borrowing moisture from successive vertical levels below until all specific humidity values in the column are nonnegative. Any moisture which must be borrowed from the surface does not affect the hydrological budget there.

\section{Sampling Frequency}

For the AMIP simulation, the model history is written every 6 hours.

\section{Dynamical/Physical Properties}

\section{Atmospheric Dynamics}

Primitive-equation dynamics are expressed in terms of vorticity, divergence, temperature, the logarithm of surface pressure, and specific humidity. Variations of the gas constant and specific heat capacity with water vapor content are also included.

\section{Diffusion}

- Sixth-order $\left(\nabla^{6}\right)$ hyperdiffusion is applied in spectral space to vorticity, divergence, temperature, and moisture on the hybrid coordinate surfaces (see Vertical Representation). A correction is also applied to the temperature term to approximate dissipation on constant pressure surfaces. The diffusion time scale is 4 hours at the horizontal truncation limit (see Horizontal Resolution), but this is successively halved on the top four model levels, beginning at approximately $73 \mathrm{hPa}$.

- Second-order vertical diffusion is applied below a hybrid model coordinate level of 0.650 to parameterize the PBL (see Planetary Boundary Layer). In addition, the 
TVD vertical advection scheme (see Vertical Representation) includes some dissipation of kinetic energy where sharp changes in gradient are encountered.

\section{Gravity-wave Drag}

Momentum transports associated with gravity waves are simulated by the method of Palmer et al. (1986), using directionally dependent subgrid-scale orographic variances obtained from the U.S. Navy dataset (cf. Joseph 1980 and see Orography). Surface stress due to gravity waves excited by stably stratified flow over irregular terrain is calculated from linear theory and dimensional considerations. Gravity-wave stress is a function of atmospheric density, low-level wind, and the Brunt-Vaisalla frequency. The vertical structure of the momentum flux induced by gravity waves is calculated from a local wave Richardson number, which describes the onset of turbulence due to convective instability and the turbulent breakdown approaching a critical level. See also Orography.

\section{Solar Constant/Cycles}

The solar constant is the AMIP-prescribed value of $1365 \mathrm{~W} \mathrm{~m}^{-2}$. Both seasonal and diurnal cycles in solar forcing are simulated. (The correct annual calendar is used, including Leap Years 1980, 1984, and 1988.)

\section{Chemistry}

Carbon dioxide concentration is the AMIP-prescribed value of $345 \mathrm{ppm}$. The specified ozone profile depends on pressure, total ozone in a column, the height of maximum concentration, latitude, longitude, and season. Total ozone is obtained from London et al. (1976) data, and the altitude of maximum concentration from Wilcox and Belmont (1977). Mie radiative parameters of five types of aerosol are provided for (concentration depending only on height) from WMO-ICSU (1984) data. Radiative effects of water vapor, carbon monoxide, methane, nitrous oxide, and oxygen are also included (see Radiation).

\section{Radiation}

- Atmospheric radiation is simulated after Morcrette (1989, 1990, 1991). Absorption by water vapor, ozone, carbon monoxide, carbon dioxide, methane, nitrous oxide, and 


\section{UGAMP}

oxygen is accounted for, with shortwave/longwave absorption coefficients calculated from line parameters of Rothman et al. (1983).

- For clear-sky conditions, shortwave radiation is modeled by a two-stream formulation in two spectral wavelength intervals ( 0.25 to 0.68 micron and 0.68 to 4.0 microns), using a photon path distribution method to separate the contributions of scattering and absorption processes to radiative transfer. Rayleigh scattering and Mie scattering/ absorption by five aerosol types (see Chemistry) are treated by a delta-Eddington approximation.

- The clear-sky longwave scheme employs a broad-band flux emissivity method in six spectral intervals between wavenumbers 0 and $2.6 \times 10^{5} \mathrm{~m}^{-1}$, with continuum absorption by water vapor included between wavenumbers $3.5 \times 10^{4}$ to $1.25 \times 10^{5} \mathrm{~m}^{-1}$. The temperature/pressure dependence of longwave gaseous absorption follows Morcrette et al. (1986). Aerosol absorption is also modeled by an emissivity formulation.

- Shortwave scattering and absorption by cloud droplets are treated by a deltaEddington approximation; radiative parameters include optical thickness, singlescattering albedo linked to cloud liquid water path, and prescribed asymmetry factor. Cloud types are distinguished by also defining shortwave optical thickness as a function of effective droplet radius. Clouds are treated as graybodies in the longwave, with emissivity depending on cloud liquid water path after Stephens (1978b). Longwave scattering by cloud droplets is neglected, and droplet absorption is modeled by an emissivity formulation in terms of the cloud liquid water path. For purposes of the radiation calculations, clouds of different types are treated as randomly overlapped in the vertical; convective cloud and the same type of nonconvective cloud in adjacent layers are treated as fully overlapped.

- The full radiation calculations are performed every 3 hours on a reduced horizontal grid (every fourth point in longitude only), but with effective transmissivities and emissivities returned on the T42 Gaussian grid (see Horizontal Resolution). For intermediate time steps, the effective transmissivities are scaled by the instantaneous incoming solar radiation to represent correctly the diurnal cycle; the effective emissivities are scaled by the instantaneous Planck function to treat temperature variations. However, the influence of clouds remains fixed between full-radiation steps. See also Cloud Formation. 


\section{Convection}

- Convection follows the scheme of Betts and Miller (1994), and consists of a relaxed convective adjustment towards calculated temperature and humidity reference profiles based on observations. The relaxation times are 2 hours for precipitating deep convection and 4 hours for nonprecipitating shallow convection, regarded as mutually exclusive processes. The convection is treated as shallow if the cloud top, defined by the level of nonbuoyancy, is below about $725 \mathrm{hPa}$ for land or $810 \mathrm{hPa}$ for ocean (for a surface pressure of $1000 \mathrm{hPa}$ ), or if there is insufficient moisture for precipitation to form with deep convection. Otherwise, the deep-convection scheme (including the possibility of midlevel convection with a cloud base above $725 \mathrm{hPa}$ ) is operative.

- The temperature and humidity reference profiles for deep convection are based on relevant observational data (cf. Betts 1986). The temperature reference profile is a lapse rate that is slightly unstable with respect to the wet virtual adiabat below the freezing level, and that returns at cloud top to the moist adiabat of the cloud base. For energy conservation, this reference profile is corrected (with a second iteration) in order to zero out the vertically integrated difference between the total moist enthalpy of the environment and that of the reference profile. The humidity reference profile is derived from the temperature reference by linearly interpolating between the humidities for specified values of subsaturation pressure deficit at cloud base, freezing level, and cloud top. Below the cloud base, cooling/drying by convective downdrafts is parameterized by specifying reference profiles for air parcels originating near $850 \mathrm{hPa}$ that descend at constant subsaturation and equivalent potential temperature.

- Nonprecipitating shallow convection is parameterized as a mixing of enthalpy and moisture of air below cloud base with air at and just above the capping inversion top. The reference profile is a mixing line structure joining the conserved saturation pressure and potential temperature points of all mixtures of the two sources of air (cf. Betts 1983, 1986). Reference temperature and humidity profiles are computed after specifying a partial degree of mixing within the cloud, and mixing that is a function of the inversion strength at cloud top. Cf. Betts and Miller (1994) and Slingo et al. (1994) for further details. See also Cloud Formation and Precipitation. 


\section{Cloud Formation}

- Cloud formation is simulated following the diagnostic method of Slingo (1987). Clouds are of three types: shallow and deep convective cloud (see Convection); cloud associated with fronts/tropical disturbances that forms in low, middle, or high vertical layers; and low cloud associated with temperature inversions.

- The fraction of shallow convective cloud (typically about 0.30 ) is related to the moisture tendencies within the cloud layer (cf. Betts and Miller 1994). The fraction of deep convective cloud (ranging between 0.20 to 0.80 ) is determined from the scaled convective precipitation rate (see Precipitation). If deep convective cloud forms above $400 \mathrm{hPa}$ and the fractional area is $>0.4$, anvil cirrus and shallow convective cloud also form.

- Frontal cloud is present only when the local relative humidity is $>80$ percent, the amount being a quadratic function of this humidity excess. Low frontal cloud is absent in regions of grid-scale subsidence, and the amount of low and middle frontal cloud is reduced in dry downdrafts around subgrid-scale convective clouds. Low cloud forms below a temperature inversion if the relative humidity is $>60$ percent, the cloud amount depending on this humidity excess and the inversion strength. See also Radiation for treatment of cloud-radiative interactions.

\section{Precipitation}

- Precipitation is obtained from deep convection as part of the relaxed adjustment to the reference temperature and humidity profiles (see Convection). Subsequent evaporation of this precipitation is implicitly treated through inclusion of effects of convective downdrafts in the lowest three atmospheric layers. There is additional evaporation below elevated convective cloud bases that are situated above these downdraft layers.

- In the absence of convective adjustment, precipitation also results from gridscale condensation when the local specific humidity exceeds the saturated value at the ambient temperature and pressure; the amount of precipitate depends on the new equilibrium specific humidity resulting from the accompanying latent heat release. Before falling to the surface, grid-scale precipitation must saturate all layers below the condensation level by evaporation. Melting of falling snow (see Snow Cover) occurs for air temperatures $>+2^{\circ} \mathrm{C}$. See also Snow Cover. 


\section{Planetary Boundary Layer}

Vertical diffusion of momentum, heat, and moisture (proportional, respectively, to the vertical gradients of the wind, the dry static energy, and the specific humidity) is operative only below a hybrid-coordinate vertical level of 0.650 (about $650 \mathrm{hPa}$ for a surface pressure of $1000 \mathrm{hPa}$ ). The vertically varying diffusion coefficient depends on stability (bulk Richardson number) and the vertical shear of the wind, following standard mixing-length theory (cf. Louis 1979 and Louis et al. 1981). See also Diffusion, Surface Characteristics, and Surface Fluxes.

\section{Orography}

Orography is obtained from a U.S. Navy dataset (cf. Joseph 1980) with resolution of 10 minutes arc on a latitude/longitude grid. The mean terrain heights are then calculated for a T106 Gaussian grid, and the square root of the corresponding subgridscale orographic variance is added. The resulting "envelope orography" (cf. Wallace et al. 1983) is smoothed by applying a Gaussian filter with a $50 \mathrm{~km}$ radius of influence (cf. Brankovic and Van Maanen 1985). This filtered orography is then spectrally fitted and truncated at the T42 resolution of the model. See also Gravity-wave Drag.

\section{Ocean}

AMIP monthly sea surface temperature fields are prescribed and interpolated linearly in time at each time step. (These temperatures are uncorrected for nonzero surface heights associated with the spectral fitting of the topography--see Orography).

\section{Sea Ice}

AMIP monthly sea ice extents are prescribed, but ice surface temperatures are specified from the Alexander and Mobley (1976) dataset. (Points with surface temperatures $<-2{ }^{\circ} \mathrm{C}$ that are not on land are identified as sea ice; the masking procedure is described by Brugge 1993.) Snow does not accumulate on sea ice.

\section{Snow Cover}

Grid-scale precipitation falls as snow if the temperature of the cloud layer is below $0{ }^{\circ} \mathrm{C}$ and that of intervening layers is below $+2{ }^{\circ} \mathrm{C}$ (thereby inhibiting the melting of falling snow--see Precipitation). Snow depth (in meters of equivalent liquid water) is determined prognostically from a budget equation, but with accumulation 
only on land. The fractional area of a grid box covered by snow is given by the ratio of the snow depth to a critical depth $(0.015 \mathrm{~m})$, or is set to unity if the depth exceeds the critical value. Sublimation of snow is calculated as part of the surface evaporative flux (see Surface Fluxes), and snowmelt (occurring for ground temperatures $>0{ }^{\circ} \mathrm{C}$ ) contributes to soil moisture (see Land Surface Processes). Snow cover also alters the surface albedo (see Surface Characteristics).

\section{Surface Characteristics}

- The land surface is modeled as bare or with snow cover. Vegetation is not explicitly specified, but is accounted for in the prescribed surface properties described below.

- Roughness length is prescribed as $1.0 \times 10^{-3} \mathrm{~m}$ over sea ice. Over open ocean the roughness is computed from the surface wind stress following Charnock (1955), but it is constrained to be at least $1.5 \times 10^{-5} \mathrm{~m}$. The roughness length over land is prescribed as a blended function of local orographic variance (Tibaldi and Geleyn 1981), vegetation (Baumgartner et al. 1977), and urbanization (from the U.S. Navy data set described by Joseph 1980) that is interpolated to the model grid; the logarithm of local roughness length is also smoothed by the same Gaussian filter used for orography (see Orography).

- Annual means of satellite-observed surface albedo (range 0.07 to 0.80 ) from data of Preuss and Geleyn (1980) and Geleyn and Preuss (1983) are interpolated to the model grid and smoothed by the same Gaussian filter as used for orography (see Orography). Snow cover alters this background albedo, with a limiting value of 0.80 for snow depths $>>0.01 \mathrm{~m}$ equivalent water. Sea ice albedo is prescribed as 0.55 , and ocean albedo as 0.07 . All albedos are also functions of solar zenith angle.

- Longwave emissivity is prescribed as 0.996 for all surfaces. See also Sea Ice, Snow Cover, Surface Fluxes, and Land Surface Processes.

\section{Surface Fluxes}

- Surface solar absorption is determined from surface albedos, and longwave emission from the Planck equation with prescribed constant surface emissivity (see Surface Characteristics).

- Surface turbulent eddy fluxes are simulated as stability-dependent diffusive 
processes, following Monin-Obukhov similarity theory. Fluxes of momentum/heat/ moisture are calculated from bulk formulae that include the product of a drag/transfer coefficient, the low-level wind speed, and the vertical difference between winds/dry static energy/specific humidity at the surface and their values at the lowest atmospheric level (996 hPa for a surface pressure of $1000 \mathrm{hPa}$ ). The low-level wind speed includes an imposed minimum of $3 \mathrm{~m} \mathrm{~s}^{-1}$ and an additional $3 \mathrm{~m} \mathrm{~s}^{-1}$ (added quadratically) in the presence of convection. (The former quantity increases surface fluxes in the limit of low wind speed, while the latter accounts for subgrid-scale convective circulations--cf. Slingo et al. 1994.) The surface drag/exchange coefficients are functions of stability (bulk Richardson number) and roughness length (see Surface Characteristics) following the formulation of Louis (1979) and Louis et al. (1981). The same transfer coefficient is used for the surface heat and moisture fluxes.

- The surface moisture flux is also equivalent to the potential evaporation from a saturated surface multiplied by an evapotranspiration efficiency factor $\beta$ (cf. Budyko 1974). The factor $\beta$ is specified as unity over oceans and regions of dew formation (where the lowest atmospheric level is supersaturated); otherwise, $\beta$ varies with the snow cover and soil moisture content (see Snow Cover and Land Surface Processes).

\section{Land Surface Processes}

- Soil temperature and moisture are determined by a model consisting of a surface layer $0.07 \mathrm{~m}$ thick, and middle and deep layers each of thickness $0.42 \mathrm{~m}$. Temperature and moisture are prescribed from monthly climatologies in the deep layer (cf. Brankovic and Van Maanen 1985 and Mintz and Serafini 1981), but vary prognostically in the surface/middle layers in response to diurnal and longer-period forcings.

- Soil temperature is determined by simulating heat diffusion with an upper boundary condition specified by the net balance of surface energy fluxes (see Surface Fluxes). Soil heat capacity and diffusivity are prescribed constants (density weighted heat capacity is $2.4 \times 10^{6} \mathrm{~J} \mathrm{~m}^{-3} \mathrm{~K}^{-1}$ and heat diffusivity is $7.5 \times 10^{-7} \mathrm{~m}^{2} \mathrm{~s}^{-1}$ ).

- Soil moisture also obeys a diffusion equation (with diffusivity one-seventh that of the heat diffusivity). The upper boundary condition is specified from the combined rainfall and snowmelt, and from surface evaporation that is reduced by the presence of (fractional) snow cover. Runoff occurs if the soil moisture exceeds the 


\section{UGAMP}

layer capacity (scaled according to thickness: $0.02 \mathrm{~m}$ for the surface layer and $0.12 \mathrm{~m}$ for each of the other layers). The evapotranspiration efficiency factor $\beta$ (see Surface Fluxes) is a composite of values determined for the snow-covered and bare-land fractions of a grid box. For snow-covered surfaces (see Snow Cover), $\beta$ is unity. Over bare land, $\beta$ is the ratio of the surface layer moisture to a prescribed fraction (0.75) of field capacity, but is constrained to be at most unity. There is also a temperaturedependent correction to account for limitation of evaporation due to lack of shortwave radiation. 


\section{UIUC: University of Illinois at Urbana-Champaign}

\section{AMIP Representative(s)}

Dr. Michael Schlesinger, Department of Atmospheric Sciences, University of Illinois at Urbana-Champaign, 105 South Gregory Avenue, Urbana, Illinois 61801; Phone: +1-217-333-2192; Fax: +1-217-244-4393; e-mail: schlesin@uiatma.atmos.uiuc.edu

\section{Model Designation}

\section{UIUC MLAM-AMIP 4x5L7}

\section{Model Lineage}

The UIUC multilevel atmospheric model (MLAM) traces its origins to the twolayer Oregon State University model described by Ghan et al. (1982). Subsequent modifications principally include an increase in vertical resolution from 2 to 7 layers, as well as substantial changes in the treatment of atmospheric radiation, convection, cloud/precipitation formation, and land surface processes.

\section{Model Documentation}

The dynamical structure and numerics of the UIUC model, as well as some of its surface schemes are as described by Ghan et al. (1982). The parameterizations of radiation, cloud formation, and related physics are discussed by $\mathrm{Oh}$ (1989) and by $\mathrm{Oh}$ and Schlesinger (1991a, b, c).

\section{Numerical/Computational Properties}

\section{Horizontal Representation}

Finite differences on a B-grid (cf. Arakawa and Lamb 1977), conserving total atmospheric mass, energy, and potential enstrophy.

\section{Horizontal Resolution}

$4 \times$ 5-degree latitude-longitude grid. 


\section{UIUC}

\section{Vertical Domain}

Surface to $200 \mathrm{hPa}$ (model top). For a surface pressure of $1000 \mathrm{hPa}$, the lowest prognostic level is at $990 \mathrm{hPa}$ and the highest is at $280 \mathrm{hPa}$.

\section{Vertical Representation \\ Finite-difference sigma coordinates.}

\section{Vertical Resolution}

There are 7 unevenly spaced sigma layers between the surface and the model top at $200 \mathrm{hPa}$. (Proceeding from the surface, the thicknesses of the bottom three layers are about $20 \mathrm{hPa}, 40 \mathrm{hPa}$, and $100 \mathrm{hPa}$, while the upper four layers are each $160 \mathrm{hPa}$ thick).

\section{Computer/Operating System}

For the AMIP simulation, the model was run on a Cray C90 computer using one processor in a UNICOS environment.

\section{Computational Performance}

For the AMIP experiment, about 1.25 minutes of Cray C90 computer time per simulated day.

\section{Initialization}

For the AMIP simulation, initial conditions for the atmosphere, soil moisture, and snow cover/depth for 1 January 1979 are specified from a previous model simulation of January.

\section{Time Integration Scheme(s)}

For integration of dynamics each hour, the first step by the Matsuno scheme is followed by a sequence of leapfrog steps, each of length 6 minutes. The diabatic terms (including full radiation calculations), dissipative terms, and the vertical flux convergence of the specific humidity are recalculated hourly. 


\section{Smoothing / Filling}

Orography is area-averaged on the model grid (see Orography). A longitudinal smoothing of the zonal pressure gradient and the zonal and meridional mass flux is performed at latitudes polewards of 38 degrees (cf. Ghan et al. 1982). It is unnecessary to fill spurious negative values of atmospheric moisture, since these are not generated by the numerical schemes.

\section{Sampling Frequency}

For the AMIP simulation, the model history is written every six hours.

\section{Dynamical/Physical Properties}

\section{Atmospheric Dynamics}

Primitive-equations dynamics are expressed in terms of $u$ and $v$ winds, temperature, surface pressure, and specific humidity. Cloud water is also a prognostic variable (see Cloud Formation).

\section{Diffusion}

- Horizontal diffusion is not modeled.

- Vertical diffusion of momentum, sensible heat, and moisture operates at all vertical levels. The diffusion depends on the vertical wind shear, but not on stability (cf. Oh 1989 and Oh and Schlesinger 1991a).

\section{Gravity-wave Drag}

Gravity-wave drag is not modeled.

\section{Solar Constant/Cycles}

The solar constant is the AMIP-prescribed value of $1365 \mathrm{~W} \mathrm{~m}^{-2}$. Both seasonal and diurnal cycles in solar forcing are simulated.

\section{Chemistry}

The carbon dioxide concentration is the AMIP-prescribed value of $345 \mathrm{ppm}$. The daily horizontal distribution of column-integrated ozone is interpolated from 


\section{UIUC}

prescribed monthly mean Total Ozone Mapping Spectrometer (TOMS) data (for example, cf. Stolarski et al. 1991). The radiative effects of water vapor, methane, nitrous oxide, and chlorofluorocarbon compounds CFC-11 and CFC-12 are also included, but not those of aerosols (see Radiation).

\section{Radiation}

- The spectral range of shortwave radiation is divided into three intervals: 0 to 0.44 micron, 0.44 to 0.69 micron, and 0.69 to 3.85 microns. The first two intervals are for the treatment of Rayleigh scattering (after Coakley et al. 1983) and ozone and carbon dioxide absorption (after Lacis and Hansen 1974 and Fouquart 1988, respectively); the last interval (further subdivided into six subintervals) is for water vapor absorption. Scattering and absorption by both gases and cloud droplets are modeled following a two-stream approach with delta-Eddington approximation. The optical depth and single-scattering albedo for cloud droplets are determined following Stephens (1978b) for non-ice clouds and Starr and Cox (1985) for cirrus clouds.

- The longwave flux calculations are based on a two-stream formulation with parameterized optical depths, but with scattering neglected. Longwave absorption is treated in four spectral bands, one each for carbon dioxide $\left(5.4 \times 10^{4}\right.$ to $\left.8.0 \times 10^{4} \mathrm{~m}^{-1}\right)$ and ozone $\left(9.8 \times 10^{4}\right.$ to $\left.1.1 \times 10^{5} \mathrm{~m}^{-1}\right)$, and the other two bands (with multiple subintervals between 0 and $3.0 \times 10^{5} \mathrm{~m}^{-1}$ ) for the line centers, wings, and continuum of the water vapor absorption spectra. Pressure-broadening effects are included in all the absorption calculations, which follow Chou (1984) for water vapor, Kneizys et al. (1983) for ozone, Chou and Peng (1983) for carbon dioxide, Donner and Ramanathan (1980) for methane and nitrous oxide, and Ramanathan et al. (1985) for chlorofluorocarbon compounds CFC-11 and CFC-12. The absorption by trace gases (methane, nitrous oxide, CFC-11, and CFC-12) is normalized in each subinterval. Absorption by cloud droplets is treated by an emissivity formulation based on data by Stephens (1978b) for non-ice clouds, and by Starr and Cox (1985) and Griffith et al. (1980) for extratropical and tropical cirrus clouds, respectively.

- The radiation parameterization includes cloud-cover feedback by calculating separately the radiative fluxes for the cloudy and clear portions of each grid box; it includes cloud optical-depth feedback by linking the radiative properties to the prognostic cloud water content (see Cloud Formation). Clouds are vertically distributed 
by groups that make up an ensemble of contiguous cloud layers, and which are separated from each other by at least one layer of clear air. Following Geleyn (1977), the contiguous cloud layers within each group overlap one another fully in the vertical, while the noncontiguous cloud groups overlap randomly. Cf. Oh (1989) and Oh and Schlesinger (1991c) for further details.

\section{Convection}

- Penetrative convection is simulated by the scheme of Arakawa and Schubert (1974), as implemented by Lord (1978) and Lord et al. (1982). The scheme predicts mass fluxes from mutually interacting cumulus subensembles which have different entrainment rates and levels of neutral buoyancy that define the tops of the clouds and their associated convective updrafts. In turn, the predicted convective mass fluxes feed back on the large-scale fields of temperature (through latent heating and compensating subsidence), moisture (through precipitation and detrainment), and momentum (through cumulus friction). The effects of phase changes from water to ice on convective cloud buoyancy, and the drying/ cooling of the environment by convective-scale downdrafts are also treated.

- The mass flux for each cumulus subensemble, which is assumed to originate in the planetary boundary layer ( $\mathrm{PBL}$ ) is predicted from an integral equation that includes a positive-definite work function and a negative-definite kernel which expresses the effects of other subensembles on this work function. The predicted cumulus mass fluxes are positive-definite optimal solutions of this integral equation under a quasi-equilibrium constraint (cf. Lord et al. 1982). The cumulus mass fluxes are computed by the "exact direct method" (cf. Tokioka et al. 1984).

- A moist convective adjustment process simulates midlevel convection that originates above the planetary boundary layer. When the lapse rate exceeds moist adiabatic and supersaturation occurs, mass is mixed such that either the lapse rate is restored to moist adiabatic or the supersaturation is eliminated through precipitation formation (see Precipitation). In addition, if the lapse rate becomes dry convectively unstable anywhere within the model atmosphere, moisture and enthalpy are redistributed vertically.

\section{Cloud Formation}

- The cloud parameterization is formulated separately for stratiform and cumuloform clouds, as described by Oh (1989) and Oh and Schlesinger (1991b). For both 


\section{UIUC}

cloud types, the liquid/ice water is computed prognostically, and the fractional cloud coverage of each grid box semiprognostically. The stratiform cloud fraction varies as the square root of the relative humidity. The cumuloform cloud fraction is determined as a function of the relative humidity and the convective mass flux (see Convection).

- Cloud in the PBL (see Planetary Boundary Layer) is diagnostically computed on the basis of a cloud-topped mixed layer model (cf. Lilly 1968 and Guinn and Schubert 1989).

\section{Precipitation}

- Precipitation forms via the simulated microphysical processes (autoconversion from cloud liquid/ice water) in the prognostic cloud scheme (cf. Oh 1989 and Oh and Schlesinger 1991b). 'The large-scale precipitation rate is an exponential function of the liquid water mixing ratio and the cloud water content. The difference of these quantities multiplied by the cumulus mass flux yields the convective precipitation rate (see Convection).

- The rate of evaporation of falling large-scale precipitation is proportional to the product of the rainfall rate, the relative humidity deficit from saturation, and the cloud-free fraction of the grid box. Evaporation of convective precipitation is proportional to the product of the relative humidity deficit and the cloud water content.

\section{Planetary Boundary Layer}

The top of the PBL is taken to be the height of the lowest three atmospheric layers (total thickness about $160 \mathrm{hPa}$ for a surface pressure of $1000 \mathrm{hPa}$ ). PBL cloud is diagnostically computed on the basis of a cloud-topped mixed layer model. See also Cloud Formation, Diffusion, Surface Characteristics, and Surface Fluxes.

\section{Orography}

Orography, obtained from the $1 \times 1$-degree data of Gates and Nelson (1975), is area-averaged over each $4 \times 5$-degree model grid square.

\section{Ocean}

AMIP monthly sea surface temperature fields are prescribed, with daily intermediate values determined by linear interpolation. 
Sea Ice

AMIP monthly sea ice extents are prescribed. The surface temperature of the ice is determined prognostically from the surface energy balance (see Surface Fluxes) including heat conduction from the ocean below. The conduction flux is a function of the prescribed heat conductivity and ice thickness (a constant $3 \mathrm{~m}$ ), and of the difference between the surface temperature and that of the ocean (a fixed $271.5 \mathrm{~K}$ ). When snow accumulates on sea ice, this conduction flux can contribute to snowmelt. Cf. Ghan et al. (1982) for further details.

\section{Snow Cover}

Precipitation falls as snow if the surface air temperature is $<0{ }^{\circ} \mathrm{C}$. Snow mass is determined from a prognostic budget equation that includes the rates of accumulation, melting, and sublimation. Over land, the rate of snowmelt is computed from the difference between the downward heat fluxes at the surface and the upward heat fluxes that would occur for a ground temperature equal to the melting temperature of snow $\left(0^{\circ} \mathrm{C}\right)$; snowmelt contributes to soil moisture (see Land Surface Processes). Accumulation and melting of snow may also occur on sea ice (see Sea Ice). The surface sublimation rate is equated to the evaporative flux from snow (see Surface Fluxes) unless sublimation removes all the local snow mass in less than 1 hour; in that case the sublimation rate is set equal to the snow-mass removal rate. Snow cover also alters the surface albedo (see Surface Characteristics). Cf. Ghan et al. (1982) for further details.

\section{Surface Characteristics}

- Surface roughness is specified as in Hansen et al. (1983). Over land, the roughness length is a fit to the data of Fiedler and Panofsky (1972) as a function of the standard deviation of the orography. The maximum of this value and that of the roughness of the local vegetation (including a "zero plane displacement" value for tall vegetation types--cf. Monteith 1973) determines the roughness length over land. Over sea ice, the roughness is a constant $4.3 \times 10^{-4} \mathrm{~m}$ after Doronin (1969). Over ocean, the roughness length is a function of the surface wind speed, following Garratt (1977).

- Snow-free surface albedo is updated monthly by interpolation using values for January, April, July, and October specified from data of Matthews (1983). The albedo of 


\section{UIUC}

snow-covered surfaces is determined as a linear weighted (by snow depth) interpolation of snow-free and snow-covered values. The albedo of snow is a function of its temperature (cf. Manabe et al. 1991); it also depends on solar zenith angle (cf. Briegleb and Ramanathan 1982), but not on spectral interval.

- Longwave emissivity is specified to be unity (blackbody emission) for all surfaces.

\section{Surface Fluxes}

- The absorbed surface solar flux is determined from the surface albedo, and surface longwave emission from the Planck function with constant surface emissivity of 1.0 (see Surface Characteristics).

- The turbulent surface fluxes of momentum, sensible heat, and moisture are parameterized as bulk formulae that include surface atmospheric values of winds, as well as differences between skin values of temperatures and specific humidities and their surface atmospheric values. Following Oh and Schlesinger (1990), the surface wind is taken as a fraction ( 0.7 over water and 0.8 over land and ice) of the winds extrapolated from the lowest two model layers. The surface atmospheric values of temperatures and humidities are taken to be the same as those at the lowest atmospheric level (sigma $=0.990$ ). The aerodynamic drag and transfer coefficients depend on vertical stability (bulk Richardson number) and surface roughness length (see Surface Characteristics), with the same transfer coefficient used for the fluxes of sensible heat and moisture. In addition, the surface moisture flux depends on an evapotranspiration efficiency $\beta$ that is taken as unity over snow, ice and water; over land, $\beta$ is a function of the fractional soil moisture (see Land Surface Processes).

\section{Land Surface Processes}

- Following Priestly (1959) and Bhumralkar (1975), the average ground temperature over the diurnal skin depth is computed from a prognostic budget equation whose source/sink terms include the net surface radiative flux and the sensible and latent heat fluxes (see Surface Fluxes); the thermal conductivity, volumetric heat capacity, and bulk heat capacity of snow, ice, and land are also taken into account. If the predicted ground temperature for land ice is $>0{ }^{\circ} \mathrm{C}$, the ice is implicitly assumed to melt, since the model does not include a budget equation for land ice. See also Snow Cover. 
- Soil wetness is expressed as the ratio of soil moisture content to a field capacity that is specified as a function of soil texture and surface cover after data of Vinnikov and Yeserkepova (1991). Soil wetness is determined from a prognostic budget equation that includes the rates of precipitation, snowmelt, surface evaporation, and runoff. The evapotranspiration efficiency $\beta$ over land (see Surface Fluxes) is assigned a value that is the lesser of 1.33 times the soil wetness fraction or unity. The runoff rate is a nonlinear function of the soil wetness and the combined rates of precipitation and snowmelt. If the predicted soil wetness fraction exceeds unity, the excess moisture is taken as additional runoff. 


\title{
UKMO: United Kingdom Meteorological Office
}

\author{
AMIP Representative(s) \\ Mr. Christopher Hall, Hadley Centre for Climate Prediction and Research, United \\ Kingdom Meteorological Office, London Road, Bracknell, Berkshire RG12 2SY, United \\ Kingdom; Phone: +44-344-854490; Fax: +44-344-854898; \\ e-mail: cdhall@ email.meto.govt.uk.
}

\section{Model Designation}

UKMO UM-Climate1 2.5x3.75L19

\section{Model Lineage}

The UKMO Unified Model (UM) provides a common framework for forecasting and climate applications (cf. Cullen 1993). The dynamical formulations are those described by Bell and Dickinson (1987); the physical parameterizations are substantially modified from those of an earlier UKMO model documented by Slingo (1985).

\section{Model Documentation}

Cullen (1993) gives an overview of the UKMO Unified Model. Key documentation of different model features is provided by Cullen (1991), Cullen et al. (1991), Ingram (1993), Gregory (1990), Gregory and Smith (1990), Smith (1990a, b), Smith(1993), Smith and Gregory (1990), Wilson (1989), and Wilson and Swinbank (1989).

\section{Numerical/Computational Properties}

\section{Horizontal Representation}

Fourth-order finite differences on a B-grid (cf. Arakawa and Lamb 1977, Bell and Dickinson 1987) in spherical polar coordinates. Mass-weighted linear quantities are conserved, and second moments of advected quantities are conserved under nondivergent flow.

\section{Horizontal Resolution}

$2.5 \times 3.75$-degree latitude-longitude grid. 


\section{Vertical Domain}

Surface to about $5 \mathrm{hPa}$; for a surface pressure of $1000 \mathrm{hPa}$, the lowest atmospheric level is at about $997 \mathrm{hPa}$.

\section{Vertical Representation}

Finite differences in hybrid sigma-pressure coordinates after Simmons and Struefing (1981). Mass and mass-weighted potential temperature and moisture are conserved. See also Horizontal Representation.

\section{Vertical Resolution}

There are 19 unevenly spaced sigma levels. For a surface pressure of $1000 \mathrm{hPa}$, 4 levels are below $800 \mathrm{hPa}$ and 7 levels are above $200 \mathrm{hPa}$.

\section{Computer/Operating System}

The AMIP simulation was run on a Cray Y/MP computer using two processors in a UNICOS environment.

\section{Computational Performance}

For the AMIP experiment, about 4.7 minutes Cray Y/MP computation time per simulated day (about half this time being associated with output postprocessing).

\section{Initialization}

For the AMIP experiment, the model atmosphere, soil moisture, and snow cover/ depth are initialized for 1 December 1978 from a previous model simulation. Snow mass for areas of permanent land ice is initially set to $5 \times 10^{4} \mathrm{~kg} \mathrm{~m}^{-2}$. The model is then integrated forward to the nominal AMIP start date of 1 January 1979.

\section{Time Integration Scheme(s)}

Time integration proceeds mainly by a split-explicit scheme, where the solution procedure is split into "adjustment" and "advection" phases. In the adjustment phase, a forward-backward scheme that is second-order accurate in space and time is applied. The pressure, temperature, and wind fields are updated using the pressure gradient, the main part of the Coriolis terms, and the vertical advection of potential 


\section{UKMO}

temperature. In the advective phase, a two-step Heun scheme is applied. A time step of 30 minutes (including a 10-minute adjustment step) is used for integration of dynamics and physics, except for full calculation of shortwave/longwave radiation once every 3 hours. In addition, an implicit scheme is used to compute turbulent vertical fluxes of momentum, heat, and moisture in the planetary boundary layer (PBL). Cf. Cullen et al. (1991) for further details. See also Diffusion, Planetary Boundary Layer, and Surface Fluxes.

\section{Smoothing / Filling}

To prevent numerical instability, the orography is smoothed in high latitudes (see Orography), and Fourier filtering is applied to mass-weighted velocity and to increments of potential temperature and total moisture. Negative values of atmospheric moisture are removed by summing the mass-weighted positive values in each horizontal layer, and rescaling them to ensure global moisture conservation after the negative values are reset to zero.

\section{Sampling Frequency}

For the AMIP simulation, the model history is written once every 6 hours. (All average quantities in the AMIP monthly-mean standard output data are computed from samples taken at every 30 -minute time step.)

\section{Dynamical/Physical Properties}

\section{Atmospheric Dynamics}

Primitive-equation dynamics, formulated to ensure approximate energy conservation, are expressed in terms of $u$ and $v$ winds, liquid/ice water potential temperature, total water, and surface pressure (cf. White and Bromley 1988).

\section{Diffusion}

- Linear conservative horizontal diffusion is applied at fourth-order $\left(\nabla^{4}\right)$ to moisture, and at sixth-order $\left(\nabla^{6}\right)$ to winds and to liquid water potential temperature (cf. Cullen et al. 1991).

- Stability-dependent, second-order vertical diffusion of momentum and of 
conserved cloud thermodynamic and water content variables (to include the effects of cloud-water phase changes on turbulent mixing), operates only in the PBL. The diffusion coefficients are functions of the vertical wind shear (following mixing-length theory), as well as surface roughness length and a bulk Richardson number that includes buoyancy parameters for the cloud-conserved quantities (cf. Smith 1990b). See also Cloud Formation, Planetary Boundary Layer, and Surface Fluxes.

\section{Gravity-wave Drag}

The parameterization of orographic gravity-wave drag follows a modified Palmer et al. (1986) scheme, as described by Wilson and Swinbank (1989). The drag is given by the vertical divergence of the wave stress. Near the surface, the stress is equal to the product of a representative mountain wave number, the square of the wave amplitude (taken to be the subgrid-scale orographic variance--see Orography), and the density, wind, and Brunt-Vaisalla frequency evaluated in near-surface layers. At higher levels, the stress is given by this surface value weighted by the projection of the local wind on the surface wind. If this projection goes to zero, the stress is also zero; otherwise, if the minimum Richardson number falls below 0.25 , the gravity wave is assumed to break. Above this critical level, the wave is maintained at marginal stability, and a corresponding saturation amplitude is used to compute the stress.

\section{Solar Constant/Cycles}

The solar constant is the AMIP-prescribed value of $1365 \mathrm{~W} \mathrm{~m}^{-2}$. Both seasonal and diurnal cycles in solar forcing are simulated. The seasonal cycle of solar insolation is based on a 360-day year (each month 30 days in length), with the date of perihelion adjusted to minimize discrepancies (cf. Ingram 1993).

\section{Chemistry}

The carbon dioxide concentration is the AMIP-prescribed value of $345 \mathrm{ppm}$. Zonally averaged monthly ozone profiles are specified from the climatology of Keating et al. (1987) above hybrid level 0.0225 (see Vertical Representation), and from satellite data of McPeters et al. (1984) below this level. Radiative effects of water vapor and clouds, but not those of aerosol, are also included (see Radiation). 


\section{Radiation}

- The radiation schemes are as described by Ingram (1993). Incoming insolation is based on a 360-day year (see Solar Constant/Cycles). Shortwave computations follow Slingo (1985), extended to include 4 spectral intervals (with boundaries at $0.25,0.69$, $1.19,2.38$, and 4.0 microns) and the interactive cloud optical properties of Slingo (1989). Rayleigh scattering is represented by reflection of 3 percent of the incoming insolation before any interaction with the atmosphere. Shortwave absorption (by ozone, carbon dioxide, and water vapor) is treated by use of look-up tables. The effects of pressure broadening on carbon dioxide and water vapor absorption bands are included; the overlap of these bands is assumed to be random within each spectral interval.

- Calculation of longwave fluxes follows the method of Slingo and Wilderspin (1986). Absorption by water vapor, carbon dioxide, and ozone is treated in 6 spectral bands (with boundaries at $0.0,4.0 \times 10^{4}, 5.6 \times 10^{4}, 8.0 \times 10^{4}, 9.0 \times 10^{4}, 1.1 \times 10^{5}$, and $1.2 \times 10^{5} \mathrm{~m}^{-1}$ ), using exponentials for the water vapor continuum and look-up tables otherwise. The temperature dependence of e-type absorption in the water vapor continuum is after Roberts et al. (1976). Pathlengths of gaseous absorbers are scaled to account for pressure-broadening (and for carbon dioxide, temperature-broadening) effects.

- The convective cloud in each grid box is confined to a single tower (with full vertical overlap). All absorption of shortwave radiation by the convective cloud occurs in its top layer, with the remainder of the beam passing unimpeded through lower layers. In each vertical column, the shortwave radiation interacts only with 3 stratiform clouds defined by vertical domain: low (levels 1 to 5 ), middle (levels 6 to 9 ), and high (levels 10 to 18), all randomly overlapped. The cloud with the greatest area in each domain interacts with the shortwave radiation, and it is assigned the cloud water content of all the layer clouds in the domain. (Neither the shortwave nor longwave scheme allows cloud in the top layer, however.) In the longwave, similar to the formulation of Geleyn and Hollingsworth (1979), clouds in different layers are treated as fully overlapped if there is cloud in all intervening layers, while clouds separated by cloud-free layers are treated as randomly overlapped.

- Sunlight reflected from a cloud is assumed to pass directly to space without further cloud interactions, but with full gaseous absorption; the surface albedo (see Surface Characteristics) is adjusted to account for the increased absorption due to multiple reflections between clouds and the surface. Cloud shortwave optical 
properties (optical depth, single-scattering albedo, and asymmetry factor) are calculated from the cloud water path (CWP), the effective radius of the drop-size distribution ( 7 microns for water and 30 microns for ice), and the sun angle, following the Practical Improved Flux Method of Zdunkowski et al. (1980). Cloud longwave emissivity is a negative exponential function of CWP, with absorption coefficient $65 \mathrm{~m}^{2} \mathrm{~kg}^{-1}$ for ice clouds and $130 \mathrm{~m}^{2} \mathrm{~kg}^{-1}$ for water clouds. See also Cloud Formation and Precipitation.

\section{Convection}

- Moist and dry convection are both simulated by a mass-flux scheme that is based on the bulk cloud model of Yanai et al. (1973). Convection is initiated if a parcel in vertical layer $k$ has a minimum excess buoyancy $b$ that is retained in the next higher level $\mathrm{k}+1$ when entrainment effects and latent heating are included. The convective mass flux at cloud base is taken as proportional to the excess buoyancy; the mass flux increases in the vertical for a buoyant parcel, which entrains environmental air and detrains cloud air as it rises. Both updrafts and downdrafts are represented, the latter by an inverted entraining plume with initial mass flux related to that of the updraft, and with detrainment occurring over the lowest $100 \mathrm{hPa}$ of the model atmosphere.

- When the parcel is no longer buoyant after being lifted from layer $\mathrm{m}$ to layer $m+1$, it is assumed that a portion of the convective plumes has detrained in layer $m$ so that the parcel in layer $m+1$ has minimum buoyancy b. Ascent continues until a layer $\mathrm{n}$ is reached at which an undiluted (without entrainment) parcel originating from the lowest convectively active layer $k$ would have zero buoyancy, or until the convective mass flux falls below a minimum value. Cf. Gregory (1990) and Gregory and Rowntree (1990) for further details.

\section{Cloud Formation}

- The convection scheme (see Convection) determines the vertical extent of subgrid-scale convective cloud, which is treated as a single tower in each grid box. The convective cloud base is taken as the lower boundary of the first model layer at which saturation occurs, and the cloud top as the upper boundary of the last buoyant layer. The fractional coverage of each vertical column by convective cloud is a logarithmic 


\section{UKMO}

function of the mass of liquid water condensed per unit area between cloud bottom and top (cf. Gregory 1990).

- Large-scale (stratiform) cloud is prognostically determined in a similar fashion to that of Smith (1990a). Cloud amount and water content are calculated from the total moisture (vapor plus cloud water/ice) and the liquid/frozen water temperature, which are conserved during changes of state of cloud water (i.e., cloud condensation is reversible). In each grid box, these cloud-conserved quantities are assumed to vary (because of unresolved atmospheric fluctuations) according to a top-hat statistical distribution, with specified standard deviation. The mean local cloud fraction is given by the part of the grid box where the total moisture exceeds the saturation specific humidity (defined over ice if the local temperature is $<273.15 \mathrm{~K}$, and over liquid water otherwise). See also Radiation for treatment of cloud-radiative interactions.

\section{Precipitation}

- Large-scale precipitation forms in association with stratiform cloud (see Cloud Formation). For purposes of precipitation formation and the radiation calculations (see Radiation), the condensate is assumed to be liquid above $0{ }^{\circ} \mathrm{C}$, and to be ice below $-15^{\circ} \mathrm{C}$, with a liquid/ice fraction obtained by quadratic-spline interpolation for intermediate temperatures. The rate of conversion of cloud water into liquid precipitation is a nonlinear function of the large-scale cloud fraction and the cloud-mean liquid water content, following Sundqvist $(1978,1981)$ and Golding (1986). The precipitation of ice is a nonlinear function of the cloud-mean ice content, as deduced by Heymsfield (1977). Liquid and frozen precipitation also form in subgrid-scale convection (see Convection).

- Evaporation/sublimation of falling liquid/frozen precipitation are modeled after Kessler (1969) and Lin et al. (1983). Frozen precipitation that falls to the surface defines the snowfall rate (see Snow Cover). For purposes of land hydrology, surface precipitation is assumed to be exponentially distributed over each land grid box, with fractional coverage of 0.5 for large-scale precipitation and 0.1 for convective precipitation. Cf. Smith and Gregory (1990) and Dolman and Gregory (1992) for further details.

\section{Planetary Boundary Layer}

Conditions within the PBL are typically represented by the first 5 levels above the surface (centered at about $997,975,930,869$, and $787 \mathrm{hPa}$ for a surface pressure 
of $1000 \mathrm{hPa}$ ), where turbulent diffusion of momentum and cloud-conserved thermodynamic and moisture variables may occur (see Cloud Formation and Diffusion). The PBL top is defined either by the highest of these layers, or by the layer in which a modified bulk Richardson number (that incorporates buoyancy parameters for the cloud-conserved variables) exceeds a critical value of unity. Nonlocal mixing terms are included for heat and moisture. See also Surface Characteristics, Surface Fluxes, and Land Surface Processes.

\section{Orography}

Orography obtained from the U.S. Navy 10-minute resolution dataset (cf. Joseph 1980) is grid-box averaged, and is further smoothed with a 1-2-1 filter at latitudes poleward of 60 degrees. The orographic variances required by the gravity-wave drag parameterization are obtained from the same dataset (see Gravity-wave Drag).

\section{Ocean}

AMIP monthly sea surface temperature fields are prescribed, with daily values determined by linear interpolation.

\section{Sea Ice}

AMIP monthly sea ice extents are prescribed. The ice may occupy only a fraction of a grid box, and the effects of the remaining ice leads are accounted for in the surface roughness length, shortwave albedo and longwave emission, and turbulent eddy fluxes (see Surface Characteristics and Surface Fluxes). The spatially variable sea ice thickness is prescribed from climatological data. Snow falling on sea ice affects the surface albedo (see Surface Characteristics), but not the ice thickness or thermodynamic properties. Ice temperature is prognostically determined from a surface energy balance (see Surface Fluxes) that includes a conduction heat flux from the ocean below. Following Semtner (1976), the conduction flux is proportional to the difference between the surface temperature of the ice and the subsurface ocean temperature (assumed to be fixed at the melting temperature of sea ice, or $-1.8^{\circ} \mathrm{C}$ ), and the conduction flux is inversely proportional to the prescribed ice thickness. 


\section{UKMO}

\section{Snow Cover}

Surface snowfall is determined from the rate of frozen large-scale and convective precipitation in the lowest vertical layer. (Snowfall, like surface rainfall, is assumed to be distributed exponentially over each land grid box--see Precipitation.) On land only, prognostic snow mass is determined from a budget equation that accounts for accumulation, melting, and sublimation. Snow cover affects the roughness and heat conduction of the land surface, and it also alters the albedo of both land and sea ice (see Surface Characteristics). Snow melts when the temperature of the top soil/snow layer is $>0{ }^{\circ} \mathrm{C}$, the snowmelt being limited by the total heat content of this layer. Snowmelt augments soil moisture, and sublimation of snow contributes to the surface evaporative flux over land. See also Surface Fluxes and Land Surface Processes.

\section{Surface Characteristics}

- Surface types include land, ocean, sea ice, and permanent land ice. Sea ice may occupy a fraction of a grid box (see Sea Ice). On land, 15 different soil/vegetation types are specified from the $1 \times 1$-degree data of Wilson and Henderson-Sellers (1985). The effects of these surface types on surface albedo (see below) and on surface thermodynamics and moisture (see Land Surface Processes) are treated via parameters derived by Buckley and Warrilow (1988).

- On each surface, roughness lengths are specified for momentum, for heat and moisture, and for free convective turbulence (which applies in cases of very light surface winds under unstable conditions). Over oceans, the roughness length for momentum is a function of surface wind stress (cf. Charnock 1955), but is constrained to be at least $10^{-4} \mathrm{~m}$; the roughness length for heat and moisture is a constant $10^{-4} \mathrm{~m}$, and it is $1.3 \times 10^{-3} \mathrm{~m}$ for free convective turbulence. Over sea ice, the roughness length is a constant $3 \times 10^{-3} \mathrm{~m}$, but it is $0.10 \mathrm{~m}$ for that fraction of the grid box with ice leads (see Sea Ice). Over land, the roughness length is a function of vegetation and small surface irregularities; it is decreased as a linear function of snow cover, but is at least $5 \times 10^{-4} \mathrm{~m}$. Cf. Smith (1990b) for further details.

- The surface albedo of open ocean is a function of solar zenith angle. The albedo of sea ice varies between 0.60 and 0.85 as a linear function of the ice temperature above $-5^{\circ} \mathrm{C}$, and it is also modified by snow cover. Where there is partial coverage of a grid box by sea ice, the surface albedo is given by the fractionally weighted albedos 
of sea ice and open ocean. Surface albedos of snow-free land are specified according to climatological vegetation and land use. Snow cover modifies the albedo of the land surface depending (exponentially) on the depth (following Hansen et al. 1983) and (linearly) on the snow temperature above $-2{ }^{\circ} \mathrm{C}$ (following Warrilow et al. 1990), as well as on the background vegetation (following Buckley and Warrilow 1988). Cf. Ingram (1993) for further details.

- Longwave emissivity is unity (blackbody emission) for all surfaces. Thermal emission from grid boxes with partial coverage by sea ice (see Sea Ice) is calculated from the different surface temperatures of ice and the open-ocean leads, weighted by the fractional coverage of each.

\section{Surface Fluxes}

- Surface solar absorption is determined from the surface albedos, and longwave emission from the Planck equation with constant emissivity of 1.0 (see Surface Characteristics).

- Turbulent eddy fluxes are formulated as bulk formulae in a constant-flux surface layer, following Monin-Obukhov similarity theory. The momentum flux is expressed in terms of a surface stress, and the heat and moisture fluxes in terms of cloud-conserved quantities (liquid/frozen water temperature and total water content) to account for effects of phase changes on turbulent exchanges (see Cloud Formation). These surface fluxes are solved by an implicit numerical method.

- The surface atmospheric variables required for the bulk formulae are taken to be at the first level above the surface (at $997 \mathrm{hPa}$ for a $1000 \mathrm{hPa}$ surface pressure). (For diagnostic purposes, temperature and humidity at $1.5 \mathrm{~m}$ and the wind at $10 \mathrm{~m}$ are also estimated from the constant-flux assumption.) Following Louis (1979), the $\mathrm{drag} /$ transfer coefficients in the bulk formulae are functions of stability (expressed as a bulk Richardson number) and roughness length, and the same transfer coefficient is used for heat and moisture. In grid boxes with fractional sea ice, surface fluxes are computed separately for the ice and lead fractions, but using mean drag and transfer coefficients obtained from linearly weighting the coefficients for ice and lead fractions (see Sea Ice).

- The surface moisture flux also is a fraction $\beta$ of the local potential evaporation for a saturated surface. Over oceans, snow and ice, and where there is dew formation 


\section{UKMO}

over land, $\beta$ is set to unity; otherwise, $\beta$ is a function of soil moisture and vegetation (see Land Surface Processes).

- Above the surface layer, momentum and cloud-conserved variables are mixed vertically within the PBL by stability-dependent diffusion. For unstable conditions, cloud-conserved temperature and water variables are transported by a combination of nonlocal and local mixing. Cf. Smith $(1990 b, 1993)$ for further details. See also Diffusion and Planetary Boundary Layer.

\section{Land Surface Processes}

- A vegetation canopy model (cf. Warrilow et al. 1986 and Shuttleworth 1988) includes effects of moisture condensation, precipitation interception, direct evaporation from wet leaves and from surface ponding, and evapotranspiration via root uptake of soil moisture. The fractional coverage and water-storage capacity of the canopy vary spatially by vegetation type (see Surface Characteristics), with a small storage added to represent surface ponding. The canopy intercepts a portion of the precipitation, which is exponentially distributed over each grid box (see Precipitation). Throughfall of canopy condensate and intercepted precipitation occurs in proportion to the degree of fullness of the canopy; evaporation from the wet canopy occurs in the same proportion, as a fraction of the local potential evaporation. Cf. Gregory and Smith (1990) for further details.

- Soil moisture is predicted from a single-layer model with spatially nonuniform water-holding capacity; it is augmented by snowmelt, precipitation, and the throughfall of canopy condensate. This moisture infiltrates the soil at a rate depending on saturated soil hydraulic conductivity enhanced by effects of root systems that vary spatially by vegetation type (see Surface Characteristics). The noninfiltrated moisture is treated as surface runoff. Subsurface runoff from gravitational drainage is also parameterized as a function of spatially varying saturated soil hydraulic conductivity and of the ratio of soil moisture to its saturated value (cf. Eagleson 1978). Soil moisture is depleted by evaporation at a fraction $\beta$ of the local potential rate (see Surface Fluxes). The value of $\beta$ is obtained by combining the canopy evaporation efficiency (see above) with a moisture availability parameter that depends on the ratio of soil moisture to a spatially varying critical value, and of the ratio of the stomatal resistance to aerodynamic resistance (cf. Monteith 1965). The critical soil moisture and stomatal resistance vary spatially 
by soil/vegetation type (see Surface Characteristics). Cf. Gregory and Smith (1990) and Smith (1990b) for further details.

- Soil temperature is predicted after Warrilow et al. (1986) from heat conduction in four layers. The depth of the topmost soil layer is given by the penetration of the diurnal wave, which depends on the spatially varying soil heat conductivity/capacity (see Surface Characteristics). The lower soil layers are, respectively, about 3.9, 14.1, and 44.7 times the depth of this top layer. The top boundary condition for heat conduction is the net downward surface energy balance (see Surface Fluxes), including the latent heat of fusion for snowmelt (see Snow Cover); the bottom boundary condition is zero heat flux. Heat insulation by snow (see Snow Cover) is modeled by reducing the thermal conductivity between the top two soil layers; however, subsurface moisture does not affect the thermodynamic properties of the soil. Cf. Smith (1990b) for further details. 


\title{
YONU: Yonsei University
}

\author{
AMIP Representative(s) \\ Dr. Jai-Ho Oh and Dr. Jeong-Woo Kim, Department of Astronomy and Atmospheric \\ Sciences, Yonsei University, Sinchon-Dong 134, Seodaemun-Ku, Seoul, Korea 120-749; \\ Phone: +82-2-361-2688; Fax: +82-2-365-5163; e-mail: oh@crg50.atmos.uiuc.edu
}

\section{Model Designation \\ YONU Tr5.1 4x5L5}

\section{Model Lineage}

The dynamical structure and numerics of the YONU model are essentially those of the Meteorological Research Institute (MRI) model (cf. Tokioka et al. 1984); however, the YONU and MRI model differ substantially in their treatment of radiation, cloud formation, and surface processes. Some of the YONU model surface schemes also are derived from those of the two-level Oregon State University model (cf. Ghan et al. 1982).

\section{Model Documentation}

The basic model dynamical structure and numerics are as described by Tokioka et al. (1984). The radiation, cloud formation, and related physical parameterizations are documented by Oh (1989) and by Oh and Jung (1993). Descriptions of some of the surface schemes are provided by Ghan et al. (1982).

\section{Numerical/Computational Properties}

\section{Horizontal Representation}

Finite differences on a C-grid (cf. Arakawa and Lamb 1977), conserving total atmospheric mass, energy, and potential enstrophy.

\section{Horizontal Resolution}

$4 \times 5$ degree latitude-longitude grid. 


\section{Vertical Domain}

Surface to $100 \mathrm{hPa}$ (model top). For a surface pressure of $1000 \mathrm{hPa}$, the lowest prognostic vertical level is at $900 \mathrm{hPa}$ and the highest is at $150 \mathrm{hPa}$. See also Vertical Representation and Vertical Resolution.

\section{Vertical Representation}

Finite-difference modified sigma coordinates $($ sigma $=[\mathrm{P}-\mathrm{PT}] /[\mathrm{PS}-\mathrm{PT}]$, where $P$ and PS are atmospheric and surface pressure, respectively, and PT is a constant $100 \mathrm{hPa}$ ). The vertical differencing scheme is after Tokioka (1978).

\section{Vertical Resolution}

There are 5 modified sigma layers (see Vertical Representation) centered on sigma $=0.0555,0.222,0.444,0.666$, and 0.888 .

\section{Computer/Operating System}

For the AMIP simulation, the model was run on a Cray C90 computer using a single processor in a UNICOS environment.

\section{Computational Performance}

For the AMIP experiment, about 3 minutes of Cray C90 computer time per simulated day.

\section{Initialization}

For the AMIP experiment, the atmosphere, soil moisture, and snow cover/depth are initialized for 1 January 1979 from a previous model simulation.

\section{Time Integration Scheme(s)}

For integration of the dynamics each hour, the first step is by the Matsuno scheme, and then the leapfrog scheme is applied in a sequence of eight 7.5 minute steps (cf. Tokioka et al. 1984). The diabatic terms (including full radiation calculations), dissipative terms, and vertical flux convergence of the water vapor mixing ratio are calculated hourly. 


\section{Smoothing / Filling}

Orography is area-averaged (see Orography). A longitudinal smoothing of the zonal pressure gradient and the zonal and meridional mass flux also is performed (cf. Tokioka et al. 1984). The positive-definite advection scheme of Bott $(1989 \mathrm{a}, \mathrm{b})$ is adopted to prevent generation of negative moisture values.

\section{Sampling Frequency}

For the AMIP simulation, the model history is written every 6 hours.

\section{Dynamical/Physical Properties}

\section{Atmospheric Dynamics}

Primitive-equations dynamics are expressed in terms of $u$ and $v$ winds, temperature, surface pressure, and specific humidity. Cloud water is also a prognostic variable (see Cloud Formation).

\section{Diffusion}

- Horizontal diffusion of momentum (but not of other quantities) on constant sigma surfaces is treated by the method of Holloway and Manabe (1971).

- Stability-dependent vertical diffusion of momentum, sensible heat, and moisture operates at all vertical levels (cf. Oh 1989).

\section{Gravity-wave Drag}

Gravity-wave drag is not modeled.

\section{Solar Constant/Cycles}

The solar constant is the AMIP-prescribed value of $1365 \mathrm{~W} \mathrm{~m}^{-2}$. Both seasonal and diurnal cycles in solar forcing are simulated.

\section{Chemistry}

The carbon dioxide concentration is the AMIP-prescribed value of $345 \mathrm{ppm}$. The daily horizontal distribution of ozone is interpolated from prescribed monthly ozone data of Bowman (1988). The radiative effects of water vapor, methane, nitrous oxide, 
and chlorofluorocarbon compounds CFC-11 and CFC-12 are also included, but not those of aerosols (see Radiation).

\section{Radiation}

- Shortwave radiation is calculated in three intervals: 0 to 0.44 micron, 0.44 to 0.69 micron, and 0.69-3.85 microns. The first two intervals are for the treatment of Rayleigh scattering (after Coakley et al. 1983) and ozone and carbon dioxide absorption (after Lacis and Hansen 1974 and Fouquart 1988); the last interval (further subdivided into six subintervals) is for water vapor absorption. Scattering and absorption by gases and cloud droplets are modeled by a two-stream method with use of a delta-Eddington approximation.

- The longwave calculations are based on a two-stream formulation with parameterized optical depths, but with scattering neglected. Longwave absorption is calculated in 4 intervals between 0 and $3 \times 10^{5} \mathrm{~m}^{-1}$ (one each for the carbon dioxide and ozone bands, and the other two intervals for the line centers and wings within the water vapor bands). Absorption calculations follow Chou (1984) and Kneizys et al. (1983) for water vapor, Chou and Peng (1983) for carbon dioxide, Donner and Ramanathan (1980) for methane and nitrous oxide, and Ramanathan et al. (1985) for chlorofluorocarbon compounds CFC-11 and CFC-12. The absorption by trace gases (methane, nitrous oxide, CFC-11 and CFC-12) is normalized in each subinterval. Pressure-broadening effects are included in all cases.

- The cloud radiative properties are tied to the prognostic cloud water content (see Cloud Formation). In the shortwave, the optical depth and single-scattering albedo of cloud droplets follow parameterizations of Stephens (1978b) for liquid water and Starr and $\operatorname{Cox}$ (1985) for ice. Longwave absorption by cloud droplets follows emissivity formulations of Stephens (1978b) for liquid-water clouds, and of Starr and Cox (1985) and Griffith et al. (1980) for extratropical and tropical cirrus clouds, respectively. Clouds are vertically distributed by cloud groups that make up an ensemble of contiguous cloud layers, and that are separated from each other by at least one layer of clear air. Following Geleyn (1977), the contiguous cloud layers within each group overlap fully in the vertical, while the noncontiguous cloud groups overlap randomly. Cf. $\mathrm{Oh}(1989)$ and $\mathrm{Oh}$ and Jung (1993) for further details. 


\section{YONU}

\section{Convection}

- Penetrative convection is simulated by the scheme of Arakawa and Schubert (1974), as implemented by Lord (1978) and Lord et al. (1982). The convective mass fluxes are predicted from mutually interacting cumulus subensembles which have different entrainment rates and levels of neutral buoyancy (depending on the properties of the large-scale environment) that define the tops of the clouds and their associated convective updrafts. In turn, the predicted convective mass fluxes feed back on the large-scale fields of temperature (through latent heating and compensating subsidence), moisture (through precipitation and detrainment), and momentum (through cumulus friction). The effects on convective cloud buoyancy of phase changes from water to ice are accounted for, but the drying and cooling effects of convective-scale downdrafts on the environment are not.

- The mass flux for each cumulus subensemble, assumed to originate in the planetary boundary layer (PBL), is predicted from an integral equation that includes a positive-definite work function (defined by the tendency of cumulus kinetic energy for the subensemble) and a negative-definite kernel which expresses the effects of other subensembles on this work function. The predicted cumulus mass fluxes are positivedefinite optimal solutions of this integral equation under the constraint that the rate of generation of conditional convective instability by the large-scale environment is balanced by the rate at which the cumulus subensembles suppress this instability via large-scale feedbacks (cf. Lord et al. 1982). The cumulus mass fluxes are computed by the "exact direct method," which guarantees an exact solution within roundoff errors (cf. Tokioka et al. 1984).

- A moist convective adjustment process simulates midlevel convection that originates above the planetary boundary layer. When the lapse rate exceeds moist adiabatic and supersaturation occurs, mass is mixed such that either the lapse rate is restored to moist adiabatic or the supersaturation is eliminated by formation of largescale precipitation (see Precipitation). In addition, if the lapse rate becomes dry convectively unstable anywhere within the model atmosphere, moisture and enthalpy are redistributed vertically.

\section{Cloud Formation}

- For both stratiform and cumuloform cloud types, the liquid/ice water is computed prognostically, and the fractional cloud coverage of each grid box semiprognostically. 
The vertical transport of cloud water is neglected. Following Sundqvist (1988), the fraction of stratiform cloud is determined from the relative humidity, which represents prior fractional cloud cover and liquid water content, as well as large-scale moisture convergence. The cumuloform cloud fraction is a function of convective mass flux.

- Cloud in the PBL (see Planetary Boundary Layer) is semiprognostically computed on the basis of a cloud-topped mixed layer model (cf. Lilly 1968 and Guinn and Schubert 1989). This cloud is assumed to fill the grid box (cloud fraction =1), and the computed cloud liquid water content is added to the prognostic value of cloud water if there is previous cloud formation. Cf. Oh (1989) for further details. See also Radiation for cloud-radiative interactions.

\section{Precipitation}

Precipitation is by simulation of microphysical processes (autoconversion from cloud liquid/ice water) in the prognostic stratiform and cumuloform cloud scheme (see Cloud Formation). Precipitation from cumuloform cloud is calculated in terms of convective mass flux, layer thickness, and cloud water content. Both types of precipitation may evaporate on falling through an unsaturated environment. Cf. Schlesinger et al. (1988) and Oh (1989) for further details. See also Snow Cover.

\section{Planetary Boundary Layer}

The top of the PBL is taken to be the height of the lowest atmospheric level (at sigma $=0.777$ ). The PBL is assumed to be well-mixed by convection (see Convection), and PBL cloud is simulated by a semiprognostic scheme based on a cloud-topped mixed layer model. See also Cloud Formation, Diffusion, and Surface Fluxes.

\section{Orography}

Raw orography, obtained from the $1 \times 1$-degree data of Gates and Nelson (1975), is area-averaged over each $4 \times 5$-degree model grid box. For specification of surface roughness lengths (see Surface Characteristics), the standard deviation of the $1 \times 1$-degree orography over each grid box is also determined. Cf. Ghan et al. (1982) for further details.

\section{Ocean}

AMIP monthly sea surface temperature fields are prescribed with daily intermediate values determined by linear interpolation. 


\section{Sea Ice}

The AMIP monthly sea ice extents are prescribed. The surface temperature of sea ice is predicted from the surface energy balance (see Surface Fluxes) plus heat conduction from the underlying ocean that is a function of the ice thickness (a uniform $3 \mathrm{~m}$ ) and of the difference between the ice surface temperature and that of the ocean below (fixed at $271.5 \mathrm{~K}$ ). Snow is allowed to accumulate on sea ice. When this occurs, the conduction heat flux as well as the surface energy balance can contribute to the melting of snow (see Snow Cover). Cf. Ghan et al. (1982) for further details.

\section{Snow Cover}

Precipitation falls as snow if the surface air temperature is $<0^{\circ} \mathrm{C}$. Snow mass is predicted from a budget that includes the rates of snowfall, snowmelt, and sublimation. Over land, the snowmelt (which contributes to soil moisture) is computed from the difference between the downward surface heat fluxes and the upward heat fluxes that would occur for a ground temperature of $0{ }^{\circ} \mathrm{C}$. Melting of snow on sea ice is also affected by the conduction heat flux from the ocean (see Sea Ice). (If the predicted ground temperature is $>0{ }^{\circ} \mathrm{C}$, melting of land ice is assumed implicitly, since the model does not include a land ice budget.) The surface sublimation rate is equated to the evaporative flux from snow (see Surface Fluxes) unless all the local snow is removed in less than 1 hour; in that case, the sublimation rate is equated to the snowmass removal rate. Snow cover also alters the surface albedo (see Surface Characteristics). Cf. Ghan et al. (1982) for further details. See also Land Surface Processes.

\section{Surface Characteristics}

- Surface roughness is specified as in Hansen et al. (1983). Over land, the local roughness length is the maximum of the value fitted as in Fiedler and Panofsky (1972) from the standard deviation of the orography in each grid box (see Orography) and the roughness of the local vegetation (including a zero-plane displacement for tall vegetation types--cf. Monteith 1973). The roughness length over sea ice is a constant $4.3 \times 10^{-4} \mathrm{~m}$ after estimates of Doronin (1969). Over ocean, the roughness is a function of the surface wind speed, following Garratt (1977).

- Surface albedos are specified as in Oh and Jung (1993) for nine different surface types under both snow-free and snow-covered conditions. Following Ghan et al. (1982), 
the albedo range is from 0.10 to 0.58 over land, and from 0.45 to 0.80 over ice. The albedo for the diffuse flux over oceans is 0.07 , and the direct-beam albedo depends on solar zenith angle (cf. Briegleb et al. 1986 and Payne 1972). Following Manabe and Holloway (1975), the snow-covered albedo is used if snow mass exceeds a critical value $\left(10 \mathrm{~kg} \mathrm{~m}^{-2}\right)$; otherwise, the surface albedo varies as the square-root of snow mass between snow-free and snow-covered values.

- Longwave emissivity is specified to be unity (blackbody emission) for all surfaces.

\section{Surface Fluxes}

- The absorbed surface solar flux is determined from the surface albedo, and surface longwave emission from the Planck function with constant surface emissivity of 1.0 (see Surface Characteristics).

- The turbulent surface fluxes of momentum, sensible heat, and moisture are parameterized as bulk aerodynamic formulae that include surface atmospheric values of winds, temperatures, and specific humidities in addition to ground values of the latter two variables. Following $\mathrm{Oh}$ and Schlesinger (1990), the surface wind is taken as a fraction ( 0.7 over water and 0.8 over land and ice) of the winds extrapolated from the lowest two atmospheric levels. Following Ghan et al. (1982), the surface temperature and specific humidity are obtained from a weighted mean (with respect to relative humidity) of the dry and moist adiabatic lapse rates.

- The drag and transfer coefficients in the bulk formulae depend on vertical stability (bulk Richardson number) and surface roughness length (cf. Louis 1979), with the same transfer coefficient used for the sensible heat and moisture fluxes (see Surface Characteristics). The surface moisture flux also depends on an evapotranspiration efficiency $\beta$ that is a function of the fractional soil moisture (see Land Surface Processes), but is taken as unity over ocean, ice, and snow. Cf. Oh (1989) for further details.

\section{Land Surface Processes}

- Following Priestly (1959) and Bhumralkar (1975), the average ground temperature over the diurnal penetration depth is predicted from the net balance of surface energy 
fluxes (see Surface Fluxes); the thermal conductivity, volumetric heat capacity, and bulk heat capacity of snow, ice, and land are also taken into account.

- Soil moisture is expressed as a fraction of a field capacity that is everywhere prescribed as $0.15 \mathrm{~m}$ of water in a single layer (i.e., a "bucket" model). Fractional soil moisture is predicted from a budget that includes the rates of precipitation and snowmelt, the surface evaporation, and the runoff. The evapotranspiration efficiency $\beta$ over land (see Surface Fluxes) is specified as the lesser of twice the fractional soil moisture or unity. Runoff is given by the product of the fractional soil moisture and the sum of precipitation and snowmelt rates. If the predicted fractional soil moisture exceeds unity, the excess is taken as additional runoff. Cf. Ghan et al. (1982) for further details. 


\section{References}

Abramopoulos, F., C. Rosenzweig, and B. Choudhury, 1988: Improved ground hydrology calculations for global climate models (GCMs): Soil water movement and evapotranspiration. J. Climate, 1, 921-941.

Alexander, R.C., and R.L. Mobley, 1976: Monthly average sea-surface temperatures and ice-pack limits on a $1^{\circ}$ global grid. Mon. Wea. Rev., 104, 143-148.

Alpert, J.C., M. Kanamitsu, P.M. Caplan, J.G. Sela, G.H. White, and E. Kalnay, 1988: Mountain induced gravity wave drag parameterization in the NMC mediumrange model. Preprints of the Eighth Conference on Numerical Weather Prediction, American Meteorological Society, Baltimore, MD, 726-733.

Anthes, R.A., 1977: A cumulus parameterization scheme utilizing a one-dimensional model. Mon. Wea. Rev., 105, 270-286.

Arakawa, A., 1972: Design of the UCLA general circulation model. Tech. Report No. 7, Department of Meteorology, University of California, Los Angeles, $116 \mathrm{pp}$.

Arakawa, A., and J.-M. Chen, 1987: Closure assumptions in the cumulus parameterization problem. Short-and Medium-Range Numerical Weather Prediction (Special Volume of J. Meteor. Soc. Japan), T. Matsuno (ed.), 107-131.

Arakawa, A., A. Katayama, and Y. Mintz, 1969: Numerical simulation of the general circulation of the atmosphere. Proceedings of the WMO/IUGG Symposium on Numerical Weather Prediction (Tokyo, 1968), Japan Meteorological Agency, Tokyo, pp. IV-7 to IV-12.

Arakawa, A., and V.R. Lamb, 1977: Computational design of the basic dynamical processes of the UCLA general circulation model. In Methods in Computational Physics, 17, J. Chang (ed.), Academic Press, New York, 173-265.

Arakawa, A., and V.R. Lamb, 1981: A potential enstrophy and energy conserving scheme for the shallow water equations. Mon. Wea. Rev., 109, 18-36.

Arakawa, A., and Y. Mintz, 1974: The UCLA general circulation model. Notes from a Workshop on Atmospheric Modeling, 25 March-4 April 1974, Dept. of Meteorology, University of California at Los Angeles, $404 \mathrm{pp}$.

Arakawa, A., and W.H. Schubert, 1974: Interaction of a cumulus cloud ensemble with the large scale environment, Part I. J. Atmos. Sci., 31, 674-701. 
Arakawa, A., and M.J. Suarez, 1983: Vertical differencing of the primitive equations in sigma coordinates. Mon. Wea. Rev., 111, 34-45.

Ariel, N.Z., and A.V. Murashova, 1981: Computing the adjusted nomograms for resistance coefficients of heat and moisture exchange above the sea. Trudy GGO (MGO Proc.) No 454, 9-23 (in Russian).

Asselin, R., 1972: Frequency filter for time integrations. Mon. Wea. Rev., 100, 487490.

Bader, D.C., M.C. MacCracken, and R.C. Malone, 1992: The Computer Hardware, Advanced Mathematics and Model Physics (CHAMPP) Program. Proceedings of the Third Symposium on Global Change Studies, American Meteorological Society, Atlanta, GA, 140-142.

Barkstrom, B.R., E.F. Harrison, and R.B. Lee, III, 1990: Earth Radiation Budget Experiment: Preliminary seasonal results. EOS Transactions, American Geophysical Union, 71(9), $297 \mathrm{ff}$.

Bartman, F.L., 1980: A time variable model of Earth's albedo. NASA Contract Report 159259, NASA Grant MSG 1482, University of Michican, Ann Arbor, MI.

Bath, L.M., M.A. Dias, D.L. Williamson, G.S. Williamson, and R.J. Wolski, 1987a: Users' guide to NCAR CCM1. NCAR Tech. Note NCAR/TN-286+IA, National Center for Atmospheric Research, Boulder, CO, $173 \mathrm{pp}$.

Bath, L.M., M.A. Dias, D.L. Williamson, G.S. Williamson, and R.J. Wolski, 1987b: Documentation of NCAR CCM1 program modules. NCAR Tech. Note NCAR/TN287+IA, National Center for Atmospheric Research, Boulder, CO, 307 pp.

Bath, L.M., J. Rosinski, and Jerry Olson, 1992: User's guide to NCAR CCM2. NCAR Tech. Note NCAR/TN-379+IA, National Center for Atmospheric Research, Boulder, CO, $156 \mathrm{pp}$.

Bauer, H., E. Heise, J. Pfaendtner, and V. Renner, 1985: Development of an economical soil model for climate simulation. In Current Issues in Climate Research (Proceedings of the EC Climatology Programme Symposium, held 2-5 Oct. 1984, in Sophia Antipolis, France), A. Ghazi and R. Fantechi (eds.), D. Reidel, Dordrecht, 219-226.

Baumgartner, A., H. Mayer and W. Metz, 1977: Weltweite Verteilung des Rauhigkeitsparameters $\mathrm{z}_{0}$ mit Anwendung auf die Energiedissipation an der Erdoberflaesche. Meteorolog. Rdsch., 30, 43-48. 
Beland, M., and C. Beaudoin, 1985: A global spectral model with a finite element formulation for the vertical discretization: adiabatic formulation. Mon. Wea. Rev., 113, 1910-1919.

Bell, R.S., and A. Dickinson, 1987: The Meteorological Office operational numerical weather prediction system. Met. Office Sci. Paper No. 41, United Kingdom Meteorological Office, Bracknell, Berkshire RG12 2SZ, UK.

Benoit, R., J. Cote, and J. Mailhot, 1989: Inclusion of a TKE boundary layer parameterization in the Canadian regional finite-element model. Mon. Wea. Rev., 117, 1726-1750.

Betts, A.K., 1983: Thermodynamics of mixed stratocumulus layers: Saturation point budgets. J. Atmos. Sci., 2655-2670.

Betts, A.K., 1986: A new convective adjustment scheme. Part I: Observational and theoretical basis. Quart. J. Roy. Meteor. Soc., 112, 677-691.

Betts, A.K., and Harshvardhan, 1987: Thermodynamic constraint on the cloud liquid water feedback in climate models. J. Geophys. Res., 92, 8483-8485.

Betts, A.K., and M.J. Miller, 1994: The Betts-Miller Scheme. In The Representation of Cumulus Convection in Numerical Models of the Atmosphere, K.A. Emanuel and D.J. Raymond (eds.), American Meteorological Society, Boston, MA.

Bhumralkar, C.M., 1975: Numerical experiments on the computation of ground surface temperature in an atmospheric general circulation model. J. Appl. Meteor., 14, 1246-1258.

Bignell, K.J., 1970: The water-vapor infra-red continuum. Quart. J. Roy. Meteor. Soc., 96, 390-403.

Blackadar, A.K., 1962: The vertical distribution of wind and turbulent exchange in a neutral atmosphere. J. Geophys. Res., 67, 3095-3102.

Blondin, C., 1989: Research on land surface parameterisation schemes at ECMWF. In Proceedings of the Workshop on Parameterisation of Fluxes over Land Surface, European Centre for Medium-Range Weather Forecasts, Reading, England. 
Blondin, C., and H. Boettger, 1987: The surface and subsurface parameterization scheme in the ECMWF forecasting system: Revision and operational assessment of weather elements. ECMWF Tech. Memo. No. 135, European Centre for Medium-Range Weather Forecasts, Reading, England.

Boer, G.J., N.A. McFarlane, R. Laprise, J.D. Henderson, and J.-P Blanchet, 1984a: The Canadian Climate Centre spectral atmospheric general circulation model. Atmos.-Ocean, 22, 397-429.

Boer, G.J., N.A. McFarlane, and R. Laprise, 1984b: The climatology of the Canadian Climate Centre general circulation model as obtained from a five-year simulation. Atmos.-Ocean, 22, 430-473.

Boer, G.J., N.A. McFarlane, and M. Lazare, 1992: Greenhouse gas-induced climate change simulated with the CCC second-generation general circulation model. $J$. Climate, 5, 1045-1077.

Borisenkov, Ye.P., and M.A. Kuznetsov, 1978: Parameterization of the interaction between the atmosphere and the ocean under stormy weather conditions as applied to models of general atmospheric circulation. Izvestiya Atm. Ocean. Phys., 14, 362-368.

Bott, A., 1989a: A positive definite advection scheme obtained by nonlinear renormalization of the advective fluxes. Mon. Wea. Rev., 117, 1006-1015.

Bott, A., 1989b: Reply in "Notes and Correspondence," Mon. Wea. Rev., 117, 26332636.

Bougeault, P., 1985: A simple parameterization of the large-scale effects of cumulus convection. Mon. Wea. Rev., 113, 2108-2121.

Bourke, R.H., and R.P. Garrett, 1987: Sea ice thickness distribution in the Arctic ocean. Cold Regions Sci. and Technol., 13, 259-280.

Bourke, W.P., 1974: A multi-level spectral model, 1: Formulation and hemispheric integrations. Mon. Wea. Rev., 102, 687-701.

Bourke, W.P., 1988: Spectral methods in climate models. In Physically-Based Modelling and Simulation of Climate and Climatic Change, Part 1. M.E. Schlesinger (ed.), Kluwer Academic Publishers, Dordrecht, 375-431. 
Bourke, W.P., B. McAvaney, K. Puri, and R. Thurling, 1977: Global modelling of atmospheric flow by spectral methods. In Methods in Computational Physics, 17, J. Chang (ed.), Academic Press, New York, 267-324.

Boville, B.A., 1984: The influence of the polar night jet on the tropospheric circulation in a GCM. J. Atmos. Sci., 41, 1132-1142.

Bowman, K.P., 1988: Global trends in total ozone. Science, 239, 48-50.

Bowman, K.P., and A.J. Krueger, 1985: A global climatology of total ozone from the Nimbus 7 Total Ozone Mapping Spectrometer. J. Geophys. Res., 90, 7967-7976.

Brankovic, C., and J. Van Maanen, 1985: The ECMWF climate system. ECMWF Tech. Memo. No. 109, European Centre for Medium-Range Weather Forecasts, Reading, England, 51 pp.

Briegleb, B.P., 1992: Delta-Eddington approximation for solar radiation in the NCAR community climate model. J. Geophys. Res., 97, 7603-7612.

Briegleb, B.P., P. Minnis, V. Ramanathan, and E. Harrison, 1986: Comparison of regional clear-sky albedos inferred from satellite observations and model computations. J. Clim. Appl. Meteor., 25, 214-226.

Briegleb, B.P., and V. Ramanathan, 1982: Spectral and diurnal variation in clear-sky planetary albedo. J. Appl. Meteor., 21, 1160-1171.

Brinkop, S., 1992: Parameterisierung von Grenzschichtwolken fuer Zirkulationsmodelle. Berichte aus dem Zentrum fuer Meeres- und Klimaforschung, Reihe A: Meteorologie, No. 2, Meteorologisches Institut der Universitaet Hamburg, 77 pp.

Broccoli, A.J., and S. Manabe, 1992: The effects of orography on midlatitude northern hemisphere dry climates. J. Climate, 5, 1181-1201.

Brugge, R., 1993: The UGAMP AMIP SST datasets. UGAMP Tech. Report No. 30, UK Universities' Global Atmospheric Modelling Programme, University of Reading, Reading RG6 2AU, England, 26 pp.

Buckley, E., and D.A. Warrilow, 1988: Derivation of land surface parameter datasets for use in the Met O 20 GCM. Met. O 20 Internal Note No. 81, United Kingdom Meteorological Office, Bracknell, Berkshire RG12 2SZ, UK.

Budyko, M.I., 1956: Heat Balance of the Earth's Surface. Gidrometeoizdat, Leningrad, $255 \mathrm{pp}$. 
Budyko, M.I., 1974: Climate and Life. Academic Press, New York, 525 pp.

Bunker, A.F., 1976: Computation of surface energy flux and annual air-sea interaction cycles of the North Atlantic ocean. Mon. Wea. Rev., 104, 1122-1140.

Burch, D.E., D. Gryvnak, and D. Williams, 1961: Infrared absorption by carbon dioxide. Contract AF19(604)-2633, AFCRL-255, Ohio State University, Columbus, $\mathrm{OH}$.

Burridge, D.M., and J. Haseler, 1977: A model for medium-range weather forecasting: adiabatic formulation. ECMWF Tech. Report No. 4, European Centre for Medium-Range Weather Forecasts, Bracknell, Berkshire, UK.

Businger, J.A., J.C. Wyngaard, I. Izumi, and E.F. Bradley, 1971: Flux profile relationships in the atmospheric surface layer. J. Atmos. Sci., 28, 181-189.

Butel, 1991: Manuel de référence du M.C.G.A., Versions M205/NMS206, LMD Note 155 version 1.0, Laboratoire de Météorologie Dynamique, Paris, France, 32 pp.

Cariolle, D., and M. Déqué, 1986: Southern hemisphere medium-scale waves and total ozone disturbances in a spectral general circulation model. J. Geophys. Res., 91, 10825-10846.

Cariolle, D., A. Lasserre-Bigorry, J.-F. Royer, and J.-F. Geleyn, 1990: A general circulation model simulation of the springtime Antarctic ozone decrease and its impact on mid-latitudes. J. Geophys. Res., 95, 1883-1898.

Cess, R.D., 1985: Nuclear war: Illustrative effects of atmospheric smoke and dust upon solar radiation. Clim. Change, 7, 237-251.

Cess, R.D., and G.L. Potter, 1987: Exploratory studies of cloud radiation forcing with a general circulation model. Tellus, 39A, 460-473.

Cess, R.D., G.L. Potter, S.J. Ghan, and W.L. Gates, 1985: The climatic effects of large injections of atmospheric smoke and dust: A study of climate feedback mechanisms with one-and three-dimensional climate models. J. Geophys. Res., 90, 12937-12950.

Chalita, S., and H. Le Treut, 1994: The albedo of temperate and boreal forest and the Northern Hemisphere climate: A sensitivity experiment using the LMD GCM. Clim. Dynam. (in press). 
Charnock, H., 1955: Wind stress on a water surface. Quart. J. Roy. Meteor. Soc., 81, 639-640.

Cheng, M.-D., and A. Arakawa, 1990: Inclusion of convective downdrafts in the Arakawa-Schubert cumulus parameterization. In Physical Processes in Atmospheric Models, D.R. Sikka and S.S. Singh (eds.), Wiley Eastern Ltd., New Delhi, India, 120-136.

Chervin, R.M., 1986: Interannual variability and seasonal climate predictability. $J$. Atmos. Sci., 43, 233-251.

Chou, M.-D., 1984: Broadband water vapor transmission functions for atmospheric IR flux computation. J. Atmos. Sci., 41, 1775-1778.

Chou, M.-D., 1986: Atmospheric solar heating rate in the water vapor bands. J. Atmos. Sci., 25, 1532-1542.

Chou, M.-D., and A. Arking, 1980: Computation of infrared cooling rates in the water vapor bands. J. Atmos. Sci., 37, 855-867.

Chou, M.-D., and L. Peng, 1983: A parameterization of the absorption in 15-micron $\mathrm{CO}_{2}$ spectral region with application to climate sensitivity studies. J. Atmos. Sci., 40, 2183-2192.

Chouinard, C., M. Beland, and N. McFarlane, 1986: A simple gravity wave drag parameterization for use in medium-range forecast models. Atmos.-Ocean, 24, 91-110.

Clapp, R.B., and G.M. Hornberger, 1978: Empirical equations for some soil hydraulic properties. Water Resourc. Res., 14, 601-604.

Clarke, R.H., 1970: Observational studies in the atmospheric boundary layer. Quart. J. Roy. Meteor. Soc., 96, 91-114.

Clarke, R.H., and G. Hess, 1975: On the relation between surface wind and pressure gradient, especially in lower latitudes. Bound. Layer Meteor., 9, 325-339.

Clary, O., 1987: A parameterization of gravity wave drag from linear theory, ENM Internal Report, 77 pp. [Available in French from the Centre National de Recherches Météorologiques, Toulouse, France.] 
Clough, S.A., F.X. Kneizys, R. Davies, R. Gemache, and R. Tipping, 1980: Theoretical line shape for $\mathrm{H}_{2} \mathrm{O}$ vapor: Application to continuum. In Atmospheric Water Vapor, T.D. Wilkerson and L.H. Ruhnke (eds.), Academic Press, New York, 695 pp.

Coakley, J.A., R.D. Cess, and F.B. Yurevich, 1983: The effect of tropospheric aerosols on the Earth's radiation budget: A parameterization for climate models. $J$. Atmos. Sci., 40, 116-138.

Coakley, J.A. and P. Chylek, 1975: The two-stream approximation in radiative transfer: Including the angle of the incident radiation. J. Atmos. Sci., 32, 409-418.

Coiffier, J., Y. Ernie, J.-F. Geleyn, J. Clochard, and F. Dupont, 1987: The operational hemispheric model at the French Meteorological Service. Short-and MediumRange Numerical Weather Prediction (Special Volume of J. Meteor. Soc. Japan), T. Matsuno (ed.), 337-345.

Colman, R.A., and B.J. McAvaney, 1991: Experiments using the BMRC general circulation model with a heat balance ocean. BMRC Research Report No. 24, Bureau of Meteorology Research Centre, Melbourne, Australia, $31 \mathrm{pp}$.

Colman, R.A., and B.J. McAvaney, 1992: Modelling of polar regions for climate change experiments. In Modelling Weather and Climate: The Third BMRC Modelling Workshop, November 1991. BMRC Research Report No. 33, Bureau of Meteorology Research Centre, Melbourne, Australia, 390-406.

Corby, C.A., A. Gilchrist, and P.R. Rowntree, 1976: The U.K. Meteorological Office 5-level general circulation model. In Methods in Computational Physics, 17, J. Chang (ed.), Academic Press, New York.

Coulson, K.L., 1959: Radiative flux from the top of a Rayleigh atmosphere. Ph.D. Dissertation, Dept. of Meteorology, University of California, Los Angeles, $176 \mathrm{pp}$.

Cox, C., and W. Munk, 1956: Slopes of the sea surface deduced from photographs of the sun glitter. Bull. Scripps Inst. Oceanog., 6, 401-488.

Cressman, G.P., 1959: An operative objective analysis scheme. Mon. Wea. Rev., 86, 293-297.

Cressman, G.P., 1960: Improved terrain effects in barotropic forecasts. Mon. Wea. Rev., 88, 327-342. 
Cullen, M.J.P., 1991: Positive definite advection scheme. Unified Model Documentation Paper No. 11, United Kingdom Meteorological Office, Bracknell, Berkshire RG12 2SZ, UK.

Cullen, M.J.P., 1993: The Unified Forecast/Climate Model. Meteor. Mag., 122, 81-94.

Cullen, M.J.P., T. Davies, and M.H. Mawson, 1991: Conservative finite difference schemes for a Unified Forecast/Climate Model. Unified Model Documentation Paper No. 10, United Kingdom Meteorological Office, Bracknell, Berkshire RG12 2SZ, UK.

Cunnold, D., F. Alyea, N. Phillips, and R. Prinn, 1975: A three dimensional dynamical chemical model of atmospheric ozone. J. Atmos. Sci., 32, 170-195.

Cunnold, D., W.P. Chu, R.A. Barnes, M.P. McCormick, and R.E. Veiga, 1989: Validation of SAGE II ozone measurements. J. Geophys. Res., 94, 8447-8460.

Curry, J.A., and G.F. Herman, 1985: Relationships between large-scale heat and moisture budgets and the occurrence of Arctic stratus clouds. Mon. Wea. Rev., 113, 1441-1457.

Davies, R., 1982: Documentation of the solar radiation parameterization in the GLAS climate model. NASA Tech. Memo. 83961, 57 pp. [Available from U.S. Department of Commerce, National Technical Information Service, 5285 Port Royal Road, Springfield, VA 22161.]

Deardorff, J.W., 1966: The counter-gradient heat flux in the lower atmosphere and in the laboratory. J. Atmos. Sci., 23, 503-506.

Deardorff, J.W., 1967: Empirical dependence of the eddy coefficient for heat upon stability above the lowest $50 \mathrm{~m}$. J. Appl. Meteor., 6, 631-643.

Deardorff, J.W., 1968: Dependence of air-sea transfer coefficients on bulk stability. $J$. Geophys. Res., 73, 2549-2557.

Deardorff, J.W., 1972: Parameterization of the planetary boundary layer for use in general circulation models. Mon. Wea. Rev., 100, 93-106.

Deardorff, J.W., 1977: A parameterization of ground-surface moisture content for use in atmospheric prediction models. J. Appl. Meteor., 16, 1182-1185.

Deardorff, J.W., 1978: Efficient prediction of ground surface temperature and moisture, with inclusion of a layer of vegetation. J. Geophys. Res., 83, 1889-1903. 
Deepak, A., and H.E. Gerber (eds.), 1983: World Climate Research Report of the Experts Meeting on Aerosols and their Climatic Effects, World Climate Research Reports of the World Meteorological Organization, Geneva, $107 \mathrm{pp}$.

Del Genio, A.D., and M.S. Yao, 1988: Sensitivity of a global climate model to the specification of convective updraft and downdraft mass fluxes. J. Atmos. Sci., 45, 2641-2668.

Del Genio, A.D., M.-S. Yao, and C.E. Wendell, 1993: GCM feedback sensitivity to interactive cloud water budget parameterizations. Preprints of the Fourth Symposium on Global Change Studies, American Meteorological Society, Anaheim, CA, 176-181.

Delsol, F., K. Miyakoda, and R.H. Clarke, 1971: Parameterized processes in the surface boundary layer of an atmospheric circulation model. Quart. J. Roy. Meteor. Soc., 97, 181-208.

Deutsches Klimarechenzentrum (DKRZ) Modellbetreuungsgruppe, 1992: The ECHAM3 atmospheric general circulation model. DKRZ Tech. Report No. 6, ISSN 0940-9237, Deutsches Klimarechenzentrum, Hamburg, Germany, 184 pp.

Dewey, K.F., 1987: Satellite-derived maps of snow cover frequency for the Northern Hemisphere. J. Clim. Appl. Meteor., 26, 1210-1229.

Dickinson, R.E., A. Henderson-Sellers, P.J. Kennedy, and M.F. Wilson, 1986: Biosphere-Atmosphere Transfer Scheme (BATS) for the NCAR Community Climate Model. NCAR Tech. Note NCAR/TN-275+STR, National Center for Atmospheric Research, Boulder, CO, 69 pp.

Dolman, A.J., and D. Gregory, 1992: The parameterization of rainfall interception in GCMs. Quart. J. Roy. Meteor. Soc., 118, 455-467.

Donner, L., and V. Ramanathan, 1980: Methane and nitrous oxide: Their effects on the terrestrial climate. J. Atmos. Sci., 37, 119-124.

Dopplick, T.G., 1974: Radiative heating in the atmosphere. In The General Circulation of the Tropical Atmosphere and Interactions with Extratropical Latitudes, Vol. 2. R.E. Newell, J.W. Kidson, D.G. Vincent, and G.J. Boer (eds.), M.I.T. Press, Cambridge, MA, 1-25.

Dorman, J.L., and P.J. Sellers, 1989: A global climatology of albedo, roughness length and stomatal resistance for atmospheric general circulation models as represented by the Simple Biosphere model (SiB). J. Appl. Meteor., 28, 833-855. 
Doronin, Yu. P., 1969: Thermal interaction of the atmosphere and the hydrosphere in the Arctic. Israel Program for Scientific Translations, Jerusalem, 244 pp. [NTIS N71-15668].

Duemenil, L., and E. Todini, 1992: A rainfall-runoff scheme for use in the Hamburg climate model. In Advances in Theoretical Hydrology: A Tribute to James Dooge, J.P. O'Kane (ed.), European Geophysical Society Series on Hydrological Sciences, Vol. 1, Elsevier Press, Amsterdam, 129-157.

Duetsch, H.U., 1971: Photochemistry of atmospheric ozone. Adv. Geophys., 15, 219322.

Duetsch, H.U., 1974: The ozone distribution in the atmosphere. Canadian J. Chem., 52, 1491-1504.

Duetsch, H.U., 1978: Vertical ozone distribution on a global scale. Pure Appl. Geophys., 116, 511-529.

Eagleson, P.S., 1978: Climate, soil and vegetation. Water Resourc. Res., 14, 705-776.

Ebisuzaki, W., and H.M. van den Dool, 1993: The Atmospheric Model Intercomparison Project at the National Meteorological Center. NMC Office Note 402, 19 pp. [Available from The National Meteorological Center, Camp Springs, MD.]

ECMWF Research Department, 1988: ECMWF forecast model, adiabatic part (2nd edition). European Centre for Medium-Range Weather Forecasts, Reading, England.

ECMWF Research Department, 1991: ECMWF forecast model, physical parameterisation (3rd edition). European Centre for Medium-Range Weather Forecasts, Reading, England.

ECMWF Technical Attachment, 1993: The description of the ECMWF/WCRP Level III-A global atmospheric data archive. European Centre for Medium-Range Weather Forecasts, Reading, England.

Eickerling, H., 1989: Parameterisierung des infraroten Strahlungstransports fuer Kohlendioxid, Wasserdampf und Ozon in einem breitbandigen Strahlungstransportmodell, Diplomarbeit, Institut fuer Meteorologie und Geophysik, Univ. Koeln, Germany.

Eliassen, A., and E. Palm, 1961: On the transfer of energy in stationary mountain waves. Geofys. Publ., 22, 1-23. 
Elsasser, W.M., 1960: Atmospheric radiation tables. In Meteorological Monographs, 4, American Meteorological Society, Boston, MA, 43 pp.

Feigelson, E.M., 1978: Preliminary radiation model of a cloudy atmosphere. Part I-Structure of clouds and solar radiation. Beitr. Phys. Atmos., 51, 203-229.

Feigelson, E.M., 1984: Radiation in a Cloudy Atmosphere. Atmospheric Sciences Library, D. Reidel, Dordrecht, 293 pp.

Fels, S.B., 1985: Radiative-dynamical interactions in the middle atmosphere. In Issues in Atmospheric and Oceanic Modeling, S. Manabe (ed.), Adv. Geophys., 28A, Academic Press, New York, 277-300.

Fels, S.B., J.T. Kiehl, A.A. Lacis, and M.D. Schwarzkopf, 1991: Infrared cooling rate calculations in operational general circulation models: Comparison with benchmark computations. J. Geophys. Res., 96, 9105-9120.

Fels, S.B., and M.D. Schwarzkopf, 1975: The simplified exchange approximation: A new method for radiative transfer calculations. J. Atmos. Sci., 37, 1475-1488.

Fels, S.B., and M.D. Schwarzkopf, 1981: An efficient, accurate algorithm for calculating $\mathrm{CO}_{2}$ 15-micron band cooling rates. J. Geophys. Res., 86(C2), 1205-1232.

Fiedler, F., and M.A. Panofsky, 1972: The geostrophic drag coefficient and the "effective" roughness length. Quart. J. Roy. Meteor. Soc., 98, 212-220.

Fjortoft, R., 1953: On the changes in the spectral distribution of kinetic energy for two-dimensional non-divergent flow. Tellus, 5, 225-230.

FNOC, 1986: Fleet Numerical Oceanographic Center's Numerical Environmental Products Manual, Vols. I and II. Fleet Numerical Oceanographic Center, Monterey, CA, 214 pp.

Forderhase, K., W.M. Washington, R:M. Chervin, V. Ramanathan, D.L. Williamson, and D.J. Knight, 1980: Lower boundary conditions for the NCAR global circulation model: Ocean surface temperatures, sea ice, snow cover, continental surface albedos and surface emissivity, subsurface continental temperatures and mountain heights. NCAR Tech. Note, NCAR/TN-157+STR, National Center for Atmospheric Research, Boulder, CO, 58 pp.

Fouquart, Y., 1988: Radiative transfer in climate modeling. In Physically-Based Modelling and Simulation of Climate and Climate Change, Part 1. M.E. Schlesinger (ed.), Kluwer Academic Publishers, Dordrecht, 223-283. 
Fouquart, Y., and B. Bonnel, 1980: Computation of solar heating of the Earth's atmosphere: A new parameterization. Beitr. Phys. Atmos., 53, 35-62.

Fox-Rabinovitz, M., H.M. Helfand, A. Hou, L.L. Takacs, and A. Molod, 1991: Numerical experiments on forecasting climate simulation and data assimilation with the new 17 layer GLA GCM. Proceedings of the Ninth Numerical Weather Prediction Conference, American Meteorological Society, Denver, CO, 506-509.

Galin, V. Ya., 1984: A treatment of radiative heating in a GCM. Preprint N75 of the DNM AN USSR, 21 pp. (in Russian).

Garand, L., 1983: Some improvements and complements to the infrared emissivity algorithm including a parameterization of the absorption in the continuum region. J. Atmos. Sci., 40, 230-244.

Garand, L., and J. Mailhot, 1990: The influence of infrared radiation in numerical weather forecasts. Preprints of the Seventh Conference on Atmospheric Radiation, American Meteorological Society, Atlanta, GA, 146-151.

Garratt, J.R., 1977: Review of drag coefficients over oceans and continents. Mon. Wea. Rev., 105, 915-929.

Gates, W.L., 1992: AMIP: The Atmospheric Model Intercomparison Project. Bull. Amer. Meteor. Soc., 73, 1962-1970.

Gates, W.L., and A.B. Nelson, 1975: A new (revised) tabulation of the Scripps topography on a one-degree global grid. Part 1: Terrain heights. Tech. Report R-12761-ARPA, The Rand Corporation, Santa Monica, CA, 132 pp.

Geleyn, J.-F., 1977: Parameterization of radiative transfer at ECMWF. Proceedings of the ECMWF 1977 Seminar on the Parameterisation of the Physical Processes in the Free Atmosphere, European Centre for Medium-Range Weather Forecasts, Reading, England, 273-345.

Geleyn, J.-F., 1981: Some diagnostics of the cloud/radiation interaction in ECMWF forecasting model. Proceedings of the ECMWF Workshop on Radiation and Cloud-radiation Interaction in Numerical Modelling, 15-17 October 1980, European Centre for Medium-Range Weather Forecasts, Reading, England, 135-162.

Geleyn, J.-F., 1987: Use of a modified Richardson number for parameterizing the effect of shallow convection: Short-and Medium-Range Numerical Weather Prediction (Special Volume of J. Meteor. Soc. Japan), T. Matsuno (ed.), 141-149. 
Geleyn, J.-F., 1988: Interpolation of wind, temperature, and humidity values from model levels to the height of measurement. Tellus, 40A, 347-351.

Geleyn, J.-F., P. Bougeault, M. Rochas, D. Cariolle, J.-P. Lafore, J-F. Royer, and J.-C. Andre, 1988: The evolution of numerical weather prediction and atmospheric modelling at the French weather service. J. Theor. Appl. Mech., 7, 87-110.

Geleyn, J.-F., A. Hense, and H.J. Preuss, 1982: A comparison of model generated radiation fields with satellite measurements. Beitr. Phys. Atmos., 55, 253-286.

Geleyn, J.-F., and A. Hollingsworth, 1979: An economical analytical method for the computation of the interaction between scattering and line absorption of radiation. Beitr. Phys. Atmos., 52, 1-16.

Geleyn, J.-F., and H.J. Preuss, 1983: A new data set of satellite-derived surface albedo values for operational use at ECMWF. Arch. Meteor. Geophys. Bioclim., Series A, 32, 353-359.

Ghan, S.J., J.W. Lingaas, M.E. Schlesinger, R.L. Mobley, and W.L. Gates, 1982: A documentation of the OSU two-level atmospheric general circulation model. Climatic Research Institute, Report No. 35, Oregon State University, Corvallis, OR, $395 \mathrm{pp}$.

Godson, W.L., 1953: The evaluation of infra-red radiative fluxes due to atmospheric water vapour. Quart. J. Roy. Meteor. Soc., 79, 367-379.

Golding, B.W., 1986: Short range forecasting over the United Kingdom using a mesoscale forecasting system. Short-and Medium-Range Numerical Weather Prediction (Special Volume of J. Meteor. Soc. Japan), T. Matsuno (ed.), 563-572.

Goldman, A., and T.G. Kyle, 1968: A comparison between statistical model and line calculation with application to the 9.6 micron ozone and the 2.7 micron water vapor. Appl. Optics, 7, 1167-1177.

Goody, R.M., 1952: A statistical model for water vapor absorption. Quart. J. Roy. Meteor. Soc., 78, 165-169.

Gordon, C.T., 1986: Boundary layer parameterizations and land surface processes in GFDL GCMs. Proceedings of the ISLSCP Conference, Rome, ESA SP-248, 23-36.

Gordon, C.T., 1992: Comparison of 30 day integrations with and without cloud-radiation interaction. Mon. Wea. Rev., 120, 1244-1277. 
Gordon, C.T., and R.D. Hovanec, 1985: A simple scheme for generating two layers of radiatively constrained effective clouds in GCM's. J. Geophys. Res., 90 (D6), 10563-10585.

Gordon, C.T., R.D. Hovanec, and W.F. Stern, 1984: Analyses of monthly mean cloudiness and their influence upon model-diagnosed radiative fluxes. J. Geophys. Res., 89, 4713-4738.

Gordon, C.T., and W.F. Stern, 1982: A description of the GFDL global spectral model. Mon. Wea. Rev., 110, 625-644.

Gordon, H.B., 1981: A flux formulation of the spectral atmospheric equations suitable for use in long term climate modelling. Mon. Wea. Rev., 109, 56-64.

Gordon, H.B., 1993: The CSIRO 4-level atmospheric general circulation model. CSIRO Division of Atmospheric Research, Tech. Paper No. 28, Commonwealth Scientific and Industrial Research Organization, PMB1, Mordialloc, Australia.

Gregory, D., 1990: Convection scheme. Unified Model Documentation Paper No. 27, United Kingdom Meteorological Office, Bracknell, Berkshire RG12 2SZ, UK.

Gregory, D., and P.R.R. Rowntree, 1990: A mass flux convection scheme with representation of cloud ensemble characteristics and stability dependent closure. Mon. Wea. Rev., 118, 1483-1506.

Gregory, D., and R.N.B. Smith, 1990: Canopy, surface, and soil hydrology. Unified Model Documentation Paper No. 25, United Kingdom Meteorological Office, Bracknell, Berkshire RG12 2SZ, UK.

Griffith, K.T., S.K. Cox, and R.C. Knollenberg, 1980: Infrared radiative properties of tropical cirrus clouds inferred from aircraft measurements. J. Atmos. Sci., 37, 1073-1083.

Guinn, T.A., and W.H. Schubert, 1989: Stratus: An interactive mixed layer model for personal computers (version 1.0). Department of Atmospheric Sciences, Colorado State University, Fort Collins, CO.

Hack, J.J., 1993: Parameterization of moist convection in the NCAR Community Climate Model (CCM2). J. Geophys. Res. (in press). 
Hack, J.J., L.M. Bath, G.W. Williamson, and B.A. Boville, 1989: Modifications and enhancements to the NCAR Community Climate Model (CCM1). NCAR Tech. Note, NCAR/TN-336+STR, NTIS PB89-215594/AS, National Center for Atmospheric Research, Boulder, CO, $97 \mathrm{pp}$.

Hack, J.J., B.A. Boville, B.P. Briegleb, J.T. Kiehl, P.J. Rasch, and D.L. Williamson, 1993a: Description of the NCAR Community Climate Model (CCM2). NCAR Tech. Note, NCAR/TN-382+STR, National Center for Atmospheric Research, Boulder, CO, $108 \mathrm{pp}$.

Hack, J.J., B.A. Boville, J.T. Kiehl, P.J. Rasch, and D.L. Williamson, 1993b: Climate statistics from the NCAR Community Climate Model (CCM2). J. Geophys. Res. (in press).

Haltiner, G.J., and R.T. Williams, 1980: Numerical Prediction and Dynamic Meteorology (second edition), John Wiley and Sons, New York, 477 pp.

Hansen, J., and J.B. Pollack, 1970: Near-infrared light scattering by terrestrial clouds. J. Atmos. Sci., 27, 265-281.

Hansen, J., G. Russell, D. Rind, P. Stone, A. Lacis, S. Lebedeff, R. Reudy, and L. Travis, 1983: Efficient three-dimensional global models for climate studies: Models I and II. Mon. Wea. Rev., 111, 609-662.

Harshvardhan, R. Davies, D.A. Randall, and T.G. Corsetti, 1987: A fast radiation parameterization for general circulation models. J. Geophys. Res., 92, 10091016.

Harshvardhan, D.A. Randall, T.G. Corsetti, and D.A. Dazlich, 1989: Earth radiation budget and cloudiness simulations with a general circulation model. J. Atmos. Sci., 40, 1922-1942.

Hart, T.L., W. Bourke, B.J. McAvaney, and B.W. Forgan, 1990: Atmospheric general circulation simulations with the BMRC global spectral model: The impact of revised physical parameterizations. J. Climate, 3, 436-459.

Hart, T.L., M.J. Gay, and W. Bourke, 1988: Sensitivity studies with the physical parameterizations in the BMRC global atmospheric spectral model. Austral. Meteor. Mag., 36, 47-60.

Harvey, L.D.D., 1988: Development of a sea-ice model for use in zonally averaged energy balance climate models. J. Climate, 1, 1221-1238. 
Helfand, H.M., 1985: A new scheme for the parameterization of the turbulent planetary boundary layer in the GLAS fourth order GCM. In Preprints of the Seventh Conference on Numerical Weather Prediction, American Meteorological Society, Montreal.

Helfand, H.M., M. Fox-Rabinovitz, L. Takacs, and A. Molod, 1991: Simulation of the planetary boundary layer and turbulence in the GLA GCM. Proceedings of the Ninth Conference on Numerical Weather Prediction, American Meteorological Society, Denver, CO, 514-517.

Helfand, H.M., and J.C. Labraga, 1988: Design of a non-singular level 2.5 second order closure model for prediction of atmospheric turbulence. J. Atmos. Sci., 45, 113-132.

Henderson-Sellers, A., and K. McGuffie, 1990: Are cloud amounts estimated from satellite sensor and conventional surface-based observations related? Int. J. Remote Sens., 11, 543-550.

Hense, A., M. Kerschgens, and E. Raschke, 1982: An economical method for computing radiative transfer in circulation models. Quart. J. Roy. Meteor. Soc., 108, 231-252

Hering, W.S., and T.R. Borden, Jr., 1965: Mean distributions of ozone density over North America, 1963-1964. Environ. Res. Paper 162, U.S. Air Force Cambridge Research Laboratory, Hanscom Field, Bedford, MA, 19 pp.

Heymsfield, A.J., 1977: Precipitation development in stratiform ice clouds: A microphysical and dynamical study. J. Atmos. Sci., 34, 367-381.

Hogan, T. F., and T. E. Rosmond, 1991: The description of the Navy operational global atmospheric prediction system's spectral forecast model. Mon. Wea. Rev., 119, 1786-1815.

Holloway, J.L., Jr., and S. Manabe, 1971: Simulation of climate by a general circulation model. I. Hydrological cycle and heat balance. Mon. Wea. Rev., 99, 335-370.

Holtslag, A.A.M., and A.C.M. Beljaars, 1989: Surface flux parameterization schemes: Developments and experiences at KNMI. Proceedings of the 1988 ECMWF Workshop on Parameterisation of Fluxes over Land Surface, European Centre for Medium-Range Weather Forecasts, Reading, England, 121-147 [also available as KNMI Sci. Rep. 88-06, De Bilt, Netherlands, 27 pp.]. 
Holtslag, A.A.M., and B.A. Boville, 1993: Local versus nonlocal boundary-layer diffusion in a global climate model. J. Climate, 6, 1825-1842.

Hoskins, B.J., 1980: Representation of the earth's topography using spherical harmonics. Mon. Wea. Rev., 108, 111-115.

Hoskins, B.J., and A.J. Simmons, 1975: A multi-layer spectral model and the semiimplicit method. Quart. J. Roy. Meteor. Soc., 101, 637-655.

Hou, Y.-T., 1991: Cloud-radiation-dynamics interaction. Ph.D. Thesis, University of Maryland at College Park, $209 \mathrm{pp}$.

Houghton, J.T., 1977: The Physics of the Atmosphere. Cambridge Univ. Press, Cambridge, England, $203 \mathrm{pp}$.

Hovis, W.A., and W.R. Callahan, 1966: Infrared reflectance spectra of igneous rocks, tuffs, and red sandstone from 0.5 to 22 microns. J. Optic. Soc. Amer., 56, 639-643.

Hummel, J.R., and R.A. Reck, 1979: A global surface albedo model. J. Appl. Meteor., 18, 239-253.

Ingram, W.J., 1993: Radiation, version 1. Unified Model Documentation Paper No. 23, United Kingdom Meteorological Office, Bracknell, Berkshire RG12 2SZ, UK.

Iwasaki, T., S. Yamada, and K. Tada, 1989a: A parameterization scheme of orographic gravity wave drag with the different vertical partitionings, Part I: Impact on medium range forecasts. J. Meteor. Soc. Japan, 67, 11-27.

Iwasaki, T., S. Yamada, and K. Tada, 1989b: A parameterization scheme of orographic gravity wave drag with the different vertical partitionings, Part II: Zonally averaged budget analyses based on transformed Eulerian-mean method. $J$. Meteor. Soc. Japan, 67, 29-41.

Jacka, T.H., 1983: A computer data base for Antarctic sea ice extent. ANARE Research Notes, 13, 54 pp.

Jarraud, M., C. Girrard, and J.-F. Geleyn, 1982: Note on a possible linearization of the vorticity equation in a primitive spectral model. Research Activities in Atmospheric and Ocean Modelling, Report No. 3, Working Group on Numerical Experimentation, Geneva. 
Joseph, D., 1980: Navy 10' global elevation values. National Center for Atmospheric Research notes on the FNWC terrain data set, National Center for Atmospheric Research, Boulder, CO, $3 \mathrm{pp}$.

Joseph, J.H., 1970: On the solar radiation fluxes in the troposphere. Solar Energy, 13, 251-261.

Joseph, J.H., W.J. Wiscombe, and J.A. Weinman, 1976: The delta-Eddington approximation for radiative flux transfer. J. Atmos. Sci., 33, 2452-2459.

Kalnay, E., R. Balgovind, W. Chao, D. Edelmann, J. Pfaendtner, L. Takacs, and K. Takano, 1983: Documentation of the GLAS fourth order general circulation model, Vol. I. NASA Tech. Memo. No. 86064, NASA Goddard Space Flight Center, Greenbelt, MD.

Kalnay, E., and M. Kanamitsu, 1988: Time schemes for strongly nonlinear damping equations. Mon. Wea. Rev., 116, 1945-1958.

Kalnay, M. Kanamitsu, and W.E. Baker, 1990: Global numerical weather prediction at the National Meteorological Center. Bull. Amer. Meteor. Soc., 71, 1410-1428.

Kalnay, E., M. Kanamitsu, J. Pfaendtner, J. Sela, M. Suarez, J. Stackpole, J. Tuccillo, L. Umscheid, and D. Williamson, 1989: Rules for the interchange of physical parameterizations. Bull. Amer. Meteor. Soc., 70, 620-622.

Kanamitsu, M., 1989: Description of the NMC global data assimilation and forecast system. Wea. and Forecast., 4, 335-342.

Kanamitsu, M., J.C. Alpert, K.A. Campana, P.M. Caplan, D.G. Deaven, M. Iredell, B. Katz, H.-L. Pan, J. Sela, and G.H. White, 1991: Recent changes implemented into the global forecast system at NMC. Wea. and Forecast., 6, 425-435.

Kanamitsu, M., K. Tada, T. Kudo, N. Sata, and S. Isa, 1983: Description of the JMA operational spectral model. J. Meteor. Soc. Japan., 61, 812-827.

Karol, I.L. (ed)., 1986: Radiative-photochemical atmospheric models. Gidrometeoizdat, Leningrad, $192 \mathrm{pp}$ (in Russian).

Katayama, A., 1972: A simplified scheme for computing radiative transfer in the troposphere. Tech. Report No. 6, Department of Meteorology, University of California, Los Angeles, CA, $77 \mathrm{pp}$. 
Katayama, A., 1978: Parameterization of the planetary boundary layer in atmospheric general circulation models. Kisyo Kenkyu Note No. 134, Meteorological Society of Japan, 153-200 (in Japanese).

Kazakov, A.L., and V.N. Lykossov, 1980: Parameterization of heat and moisture exchange during storms with application to problems of atmosphere-ocean interaction. Soviet Meteor. Hydrol., 8, 45-50.

Kazakov, A.L., and V.N. Lykossov, 1982: On parameterization of the interaction between the atmosphere and the underlying surface for numerical modelling of the atmospheric processes. Trudy Zapsinnii, 55, Gidrometeoizdat, Moscow, 3-20.

Keating, G.M., D.F. Young, and M.C. Pitts, 1987: Ozone reference models for CIRA. Adv. Space Res., 7, 105-115.

Kerschgens, M., U. Pilz, and E. Raschke, 1978: A modified two-stream approximation for computations of the solar radiation budget in a cloudy atmosphere. Tellus, 30, 429-435.

Kessler, E., 1969: On the distribution and continuity of water substance in atmospheric circulation. In Meteorological Monographs, 10, American Meteorological Society, Boston, MA.

Kiehl, J.T., and B.P. Briegleb, 1991: A new parameterization of the absorptance due to the 15 micron band system of carbon dioxide. J. Geophys. Res., 96, 9013-9019.

Kiehl, J.T., J.J. Hack, and B.P. Briegleb, 1993: The simulated earth radiation budget of the NCAR CCM2 and comparison with the earth radiation budget experiment. J. Geophys. Res. (in press).

Kiehl, J.T., R.J. Wolski, B.P. Briegleb, and V. Ramanathan, 1987: Documentation of radiation and cloud routines in the NCAR Community Climate Model (CCM1). NCAR Tech. Note NCAR/TN-288+1A, National Center for Atmospheric Research, Boulder, CO.

Kiehl, J.T., and T. Yamanouchi, 1985: A parameterization for absorption due to the A, B, and gamma oxygen bands. Tellus, 37B, 1-6.

Kinter III, J.L., J. Shukla, L. Marx, and E.K. Schneider, 1988: A simulation of the winter and summer circulations with the NMC global spectral model. J. Atmos. Sci., 45, 2486-2522. 
Kirtman, B., A. Vernekar, D. DeWitt, and J. Zhou, 1993: Impact of orographic gravity wave drag on extended-range forecasts with the COLA GCM. Atmosfera, 6, 3-23.

Kita, K., and A. Sumi, 1986: Reference ozone models for middle atmosphere. Meteorological Research Report 86-2, Division of Meteorology, Geophysical Institute, University of Tokyo, $26 \mathrm{pp}$.

Kitade, T., Y. Yanagino, N. Sato, and M. Oozeki, 1987: On an influence of forecastanalysis cycle on the forecast performance. Short-and Medium-Range Numerical Weather Prediction (Special Volume of J. Meteor. Soc. Japan), T. Matsuno (ed.), 347-357.

Kitoh, A., K. Yamazaki, and T. Tokioka, 1988: Influence of soil moisture and surface albedo changes over the African tropical rain forest on summer climate investigated with the MRI GCM-I. J. Meteor. Soc. Japan, 66, 65-86.

Kitzmiller, D.H., 1979: GMSF general ciruclation model's 12-month surface albedo dataset. Internal Report, Goddard Modeling and Simulation Facility, NASA Goddard Space Flight Center, Greenbelt, MD.

Kneizys, F.X., E.P. Shettle, W.O. Gallery, J.H. Chetwynd, Jr., L.W. Abreu, J.E.A. Selby, S.A. Clough, and R.W. Fenn, 1983: Atmospheric transmittance/radiance: Computer code LOWTRAN 6. Optical Physics Div., 7670, Hanscom AFB, Bedford, MA, 200 pp.

Kondo, J., 1975: Air-sea bulk transfer coefficients in diabatic conditions. Bound. Layer Meteor., 9, 91-112.

Kondratiev, K. Ya., 1972: Radiation processes in the atmosphere. WMO-No. 309, World Meteorological Organization, Geneva, $214 \mathrm{pp}$.

Koprova, L., and L. Uranova, 1978: Monthly averaged values of ozone content in 1964-1965. Soviet Meteor. Hydrol., 5, 49-55.

Kratz, D.P., and R.D. Cess, 1985: Solar absorption by atmospheric water vapor: A comparison of radiation models. Tellus, 37B, 53-63.

Kreitzberg, C.W., and D.J. Perkey, 1976: Release of potential instability: Part I. A sequential plume model within a hydrostatic primitive equation model. $J$. Atmos. Sci., 33, 456-475.

Kudoh, T., 1984: Normal mode initialization for the spectral model. Denshi Keisanshitu Houkoku Bessatsu, 37, 20-22 (in Japanese). 
Kukla, G., and D. Robinson, 1980: Annual cycle of surface albedo. Mon. Wea. Rev., 108, 56-58.

Kuo, H.L., 1965: On formation and intensification of tropical cyclones through latent heat release by cumulus convection. J. Atmos. Sci., 22, 40-63.

Kuo, H.L., 1974: Further studies of the parameterization of the influence of cumulus convection on large-scale flow. J. Atmos. Sci., 31; 1232-1240.

Lacis, A.A., and J. E. Hansen, 1974: A parameterization for the absorption of solar radiation in the Earth's atmosphere. J. Atmos. Sci., 31, 118-133.

Lanczos, C., 1956: Applied Analysis. Prentice Hall, London, 539 pp.

Lanczos, C., 1966: Discourse on Fourier Series. Hafner Publishing, 255 pp.

Laprise, R., and C. Girard, 1990: A spectral general circulation model using a piecewise-constant finite-element representation on a hybrid vertical coordinate system. J. Climate, 3, 32-52.

Large, W.G., and S. Pond, 1981: Open ocean momentum flux measurements in moderate to strong winds. J. Phys. Oceanog., 11, 324-336.

Laursen, L., and E. Eliasen, 1989: On the effects of the damping mechanisms in an atmospheric general circulation model. Tellus, 41A, 385-400.

Laval, K., R. Sadourny, and Y. Serafini, 1981: Land surface processes in a simplified general circulation model. Geophys. Astrophys. Fluid Dyn., 17, 129-150.

Legates, B.R., 1987: A climatology of global precipitation. Publ. Climatol., 40, 85 pp.

Leith, C.E., 1971: Atmospheric predictability and two-dimensional turbulence. J. Atmos. Sci., 28, 145-161.

Le Treut, H., and Z.-X. Li, 1991: Sensitivity of an atmospheric general circulation model to prescribed SST changes: feedback effects associated with the simulation of cloud optical properties. Clim. Dynam., 5, 175-187.

Liang, X.-Z., 1986: The design of the IAP GCM and the simulation of climate and its interseasonal variability. Ph.D. Thesis, Institute of Atmospheric Physics, Chinese Academy of Sciences, Beijing, China, 250 pp. 
Lilly, D.K., 1968: Models of cloud-topped mixed layers under a strong inversion. Quart. J. Roy. Meteor. Soc., 94, 292-309.

Lin, Y.-L., R.D. Farley, and H.D. Orville, 1983: Bulk parameterization of the snowfield in a cloud model. J. Clim. Appl. Meteor., 22, 1065-1092.

Lindzen, R.S., 1981: Turbulence and stress due to gravity wave and tidal breakdown. J. Geophys. Res., 86, 9707-9714.

Lindzen, R.S., 1984: Gravity waves in the middle atmosphere. In Dynamics of the Middle Atmosphere, J.R. Holton and T. Matsuno (eds.), D. Reidel, Dordrecht, 3-18.

Liou, K.-N., 1980: An Introduction to Atmospheric Radiation. International Geophysics Series, 25, Academic Press, New York, 392 pp.

Logan, J.A., 1985: Tropospheric ozone: Seasonal behavior, trends, and anthropogenic influences. J. Geophys. Res., 90, 10463-10482.

London, J., 1962: Mesosphere Dynamics, 3: The distribution of total ozone in the Northern Hemisphere. Final Report, Department of Meteorology/Oceanography, New York University, New York, NY.

London, J., R.D. Bojkov, S. Oltmans and J.I. Kelley, 1976: Atlas of the global distribution of total ozone, July 1957-June 1967. NCAR Tech. Note 113+STR, National Center for Atmospheric Research, Boulder, CO.

Lord, S.J., 1978: Development and observational verification of a cumulus cloud parameterization. Ph.D. Dissertation, University of California, Los Angeles, 339 pp.

Lord, S.J., and A. Arakawa, 1980: Interaction of a cumulus cloud ensemble with the large-scale environment. Part II. J. Atmos. Sci., 37, 2677-2692.

Lord, S.J., W.C. Chao, and A. Arakawa, 1982: Interaction of a cumulus cloud ensemble with the large-scale environment. Part IV: The discrete model. J. Atmos. Sci., 39, 104-113.

Louis, J.-F., 1979: A parametric model of vertical eddy fluxes in the atmosphere. Bound. Layer Meteor., 17, 187-202.

Louis, J.F., 1981: ECMWF forecast model documentation manual, Vol. 1. European Centre for Medium-Range Weather Forecasts, Reading, England, A1.21-A1.33. 
Louis, J.F. (ed.), 1984: ECMWF forecast model physical parameterisation. Research Manual No. 3, European Centre for Medium-Range Weather Forecasts, Reading, England.

Louis, J.-F., M. Tiedtke, J.-F. Geleyn, 1981: A short history of the PBL parameterisation at ECMWF. Proceedings of the ECMWF Workshop on Planetary Boundary Layer Parameterisation, November 1981, European Centre for Medium-Range Weather Forecasts, Reading, England, pp. 59-80.

Lowe, P.R. and J.M. Ficke, 1974: The computation of saturation vapor pressure. Tech. Paper No. 4-74, Environmental Prediction Research Facility, Naval Postgraduate School, Monterey, CA, $27 \mathrm{pp}$.

Lu, X.C., 1986: An efficient highly composite FFT algorithm, Chinese J. Comput. Phys., 3, 99-112.

Luther, F.M., R.G. Ellingson, Y. Fouquart, S. Fells, N. Scott and W.J. Wiscombe, 1988: Intercomparison of radiation codes in climate models (ICRCM): Longwave clear-sky results. Bull. Amer. Meteor. Soc., 69, 40-48.

Lykossov, V.N., 1990: K-theory of atmospheric turbulent planetary boundary layer and the Boussinesq generalized hypothesis. Soviet J. Num. Analy. Math. Modelling, 5, 221-240.

Machenauer, B., 1977: On the dynamics of gravity oscillations in a shallow water model with applications to normal mode initialization. Beitr. Phys. Atmos., 50, 253-271.

Magazenkov, L.N., D.A. Sheinin, 1988: Vertical approximation schemes and the wellposedness of the weather prediction problem for a baroclinic atmosphere. Meteorologia i Hydrologia, 6, 43-50 (in Russian).

Mailhot, J., and R. Benoit, 1982: A finite-element model of the atmospheric boundary layer suitable for use with numerical weather prediction models. J. Atmos. Sci., 39, 2249-2266.

Malkmus, W., 1967: Random Lorentz band models with exponential tailed $\mathrm{S}^{-1}$ line intensity distribution function. J. Optic. Soc. Amer., 57, 323-329.

Malone, R.C., L.H. Auer, G.A. Glatzmaier, and M.C. Wood, 1986: Nuclear winter: Three-dimensional simulations including interactive transport, scavenging, and solar heating of smoke. J. Geophys. Res., 91, 1039-1053. 
Manabe, S., 1969: Climate and ocean circulation. 1. The atmospheric circulation and the hydrology of the Earth's surface. Mon. Wea. Rev., 97, 739-774.

Manabe, S., and A.J. Broccoli, 1990: Mountains and arid climates of middle latitudes. Science, 247, 192-195.

Manabe, S., and D.G. Hahn, 1981: Simulation of atmospheric variability. Mon. Wea. Rev., 109, 2260-2286.

Manabe, S., and J.L. Holloway, 1975: The seasonal variation of the hydrologic cycle as simulated by a global model of the atmosphere. J. Geophys. Res., 80, 16171649.

Manabe, S., J. Smagorinsky, and R.F. Strickler, 1965: Simulated climatology of a general circulation model with a hydrologic cycle. Mon. Wea. Rev., 93, 769-798.

Manabe, S., R.J. Stouffer, M.J. Spelman, and K. Bryan, 1991: Transient response of a coupled ocean-atmosphere model to gradual changes of atmospheric $\mathrm{CO}_{2}$. Part I: Annual mean response. J. Climate, 4, 785-818.

Manabe, S., and R.F. Strickler, 1964: Thermal equilibrium of the atmosphere with a convective adjustment. J. Atmos. Sci., 21, 361-385.

Marchuk, G.I., V. Dymnikov, V. Zalesny, V. Lykossov, and V. Ya. Galin, 1984: Mathematical modeling of the general circulation of the atmosphere and ocean. Gidrometeoizdat, Moscow, 320 pp. (in Russian).

Matson, M., 1978: Winter snow-cover maps of North America and Eurasia, 19661976. National Environmental Satellite Service, Washington, D.C.

Matthews, E., 1983: Global vegetation and land use: New high-resolution data bases for climate studies. J. Clim. Appl. Meteor., 22, 474-487.

Matthews, E., 1984: Vegetation, land-use, and seasonal albedo data sets: Documentation of archived data tape. NASA Tech. Memo. 86107, National Aeronautics and Space Administration, Washington, D.C., 20 pp.

Matveev, L.T., 1984: Cloud Dynamics. Atmospheric Sciences Library, D. Reidel, Dordrecht, $340 \mathrm{pp}$.

Maykut, G.A., and N. Untersteiner, 1971: Some results from a time-dependent thermodynamic model of sea ice. J. Geophys. Res., 76, 1550-1575. 
McAvaney, B.J., W. Bourke, and K. Puri, 1978: A global spectral model for simulation of the general circulation. J. Atmos. Sci., 35, 1557-1583.

McAvaney, B.J., and R.A. Colman, 1993: The AMIP experiment: The BMRC AGCM configuration. BMRC Research Report No. 38, Bureau of Meteorology Research Centre, Melbourne, Australia, $43 \mathrm{pp}$.

McAvaney, B.J., J.R. Fraser, T.L. Hart, L.J. Rikus, W.P. Bourke, M.J. Naughton, and P. Mullenmeister, 1991: Circulation statistics from a non-diurnal seasonal simulation with the BMRC atmospheric GCM: R21L9. BMRC Research Report No. 29, Bureau of Meteorology Research Centre, Melbourne , Australia, 231 pp.

McClatchey, R.A., W. Fenn, J.E.A. Selby, F.E. Volz, and J.S. Garing, 1971: Optical properties of the atmosphere. AFGL-71-0279, Air Force Cambridge Research Laboratories, Hanscom Air Force Base, Bedford, MA, 85 pp.

McClatchey, R.A., W.S. Benedict, S.A. Clough, D.E. Burch, R.F. Calfee, K. Fox, L.S. Rothman, and J.S. Garing, 1973: AFCRL atmospheric absorption line parameters compilation. Env. Res. Paper No. 434, Air Force Cambridge Research Laboratories, Hanscom Air Force Base, Bedford, MA, 78 pp.

McCormick, M.P., R.E. Veiga, and W.P. Chu, 1992: Stratospheric ozone profile and total ozone trends derived from the SAGE and SAGE II data. Geophys. Res. Letters, 19, 269-272.

McFarlane, N.A., 1987: The effect of orographically excited gravity-wave drag on the circulation of the lower stratosphere and troposphere. J. Atmos. Sci., 44, 17751800.

McFarlane, N.A., G.J. Boer, J.-P. Blanchet, and M. Lazare, 1992: The Canadian Climate Centre second-generation general circulation model and its equilibrium climate. J. Climate, 5, 1013-1044.

McGregor, J.L., H.B. Gordon, I.G. Watterson, M.R. Dix, and L.D. Rotstayn, 1993: The CSIRO 9-level Atmospheric General Circulation Model. CSIRO Division of Atmospheric Research Tech. Paper No. 26, Commonwealth Scientific and Industrial Research Organization, PMB1, Mordialloc, Victoria 3195, Australia, 89 pp.

McPeters, R.D., D.F. Heath, and P.K. Bhartia, 1984: Averaged ozone profiles for 1979 from the NIMBUS 7 SBUV instrument. J. Geophys. Res., 89, 5199-5214. 
Meador, W., and W.R. Weaver, 1980: Two-stream approximations to radiative transfer in planetary atmospheres: A unified description of existing methods and a new improvement. J. Atmos. Sci., 37, 630-643.

Meleshko, V.P., B. Ye. Shneerov, M.E. Shvets, L.R. Dmitrieva-Arrago, G.V. Parshina, E.P. Yushina, L.N. Magazenkov, S.V. Bogachenko, and D.A. Sheinin, 1980: A hydrodynamical model of the general atmosphere circulation. Trudy GGO (MGO Proc.), No. 420, 3-33 (in Russian).

Meleshko, V.P., A.P. Sokolov, D.A. Sheinin, V.A. Lyubanskaya, P.V. Sporyshev, V.A. Matyugin, B.E. Shneerov, V.A. Govorkova, and V.M. Kattsov, 1991: An atmospheric general circulation/mixed layer ocean model for climate studies and long range weather forecasts. Meteorologia i Hydrologia, 5. (In Russian, with English translation also available.)

Mellor, G.L., and T. Yamada, 1974: A hierarchy of turbulence closure models for planetary boundary layers. J. Atmos. Sci., 31, 1791-1806.

Mellor, G.L., and T. Yamada, 1982: Development of a turbulence closure model for geophysical fluid problems. Rev. Geophys. Space Phys., 20, 851-875.

Michaud, R., 1987: Sensibilite de previsions meteorologiques a longue echeance aux anomalies de temperature superficielle des oceans. These d'Etat, Universite Paris.

Miller, M.J., A.C.M. Beljaars and T.N. Palmer, 1992: The sensitivity of the ECMWF model to the parameterization of evaporation from the tropical oceans. $J$. Climate, 5, 418-434.

Miller, M.J., T.N. Palmer, and R. Swinbank, 1989: Parameterization and influence of subgridscale orography in general circulation and numerical weather prediction models. Meteor. Atmos. Phys., 40, 84-109.

Milly, P.C.D., and P.S. Eagleson, 1982: Parameterization of moisture and heat fluxes across the land surface for use in atmospheric general circulation models. MIT Report No. 279, Massachusetts Institute of Technology, Cambridge, MA.

Mintz, Y., and Y. Serafini, 1981: Global fields of soil moisture and land-surface evapotranspiration. NASA Tech. Memo. 83907, Research Review--1980/81, NASA Goddard Space Flight Center, Greenbelt, MD, 178-180. 
Mintz, Y., and Y. Serafini, 1984: Global fields of monthly normal soil moisture as derived from observed precipitation and an estimated potential evapotranspiration. Final scientific report under NASA grant NAS 5-26, Part V, Dept. of Meteorology, University of Maryland at College Park.

Mintz, Y., and Y.V. Serafini, 1989: Global monthly climatology of soil moisture and water balance. Note Interne LMD No. 148, Laboratoire de Meteorologie Dynamique, Centre National de la Recherche Scientifique, Ecole Normale Superieure, Paris.

Miyakoda, K., D. Hembree, and R. Strickler, 1979: Cumulative results of extended forecast experiments. II. Model performance for summer cases. Mon. Wea. Rev., 107, 395-420.

Miyakoda, K., and J. Sirutis, 1977: Comparative integrations of global models with various parameterized processes of subgrid-scale vertical transports: Description of the parameterization. Beitr. Phys. Atmos. , 50, 445-488.

Miyakoda, K., and J. Sirutis, 1986: Manual of the E-physics. [Available from Geophysical Fluid Dynamics Laboratory, Princeton University, P.O. Box 308, Princeton, NJ 08542.]

Miyakoda, K., and J. Sirutis, 1990: Subgrid scale physics in 1-month forecasts. Part II: Systematic error and blocking forecasts. Mon. Wea. Rev., 118, 1065-1081.

Miyakoda, K., J. Sirutis, and J. Ploshay, 1986: One-month forecast experiments without anomaly boundary forcings. Mon. Wea. Rev., 114, 2363-2401.

Monahan, E.C., 1968: Sea spray as a function of low elevation wind speed. J. Geophys. Res., 73, 1127-1137.

Monteith, J.L., 1965: Evaporation and environment. Symp. Soc. Exptl. Biol., 19, 205234.

Monteith, J.L., 1973: Principles of Environmental Physics. Edward Arnold Publishers, $236 \mathrm{pp}$.

Monteith, J.L., 1981: Evaporation and surface temperature, Quart. J. Roy. Meteor. Soc., 107, 1-27.

Moorthi, S., and M.J. Suarez, 1992: Relaxed Arakawa-Schubert: A parameterization of moist convection for general circulation models. Mon. Wea. Rev., 120, 978. 1002. 
Morcrette, J.-J., 1984: Sur la parameterisation du rayonnement dans les modeles de la circulation generale atmospherique. Ph.D. Thesis, l'Universite des Sciences et Techniques de Lille, $373 \mathrm{pp}$.

Morcrette, J.-J., 1989: Description of the radiation scheme in the ECMWF model. ECMWF Tech. Memo. No. 165, European Centre for Medium-Range Weather Forecasts, Reading, England, 26 pp.

Morcrette, J.-J., 1990: Impact of changes to the radiation transfer parameterizations plus cloud optical properties in the ECMWF model. Mon. Wea. Rev., 118, 847873.

Morcrette, J.-J., 1991: Radiation and cloud radiative properties in the ECMWF operational weather forecast model. J. Geophys. Res., 96, 9121-9132.

Morcrette, J.-J., and Y. Fouquart, 1985: On systematic errors in parameterized calculation of longwave radiative transfer. Quart. J. Roy. Meteor. Soc., 111, 691-708.

Morcrette, J.-J., L. Smith, and Y. Fouquart, 1986: Pressure and temperature dependence of the absorption in longwave radiation parameterizations. Beitr. Phys. Atmos., 59, 455-469.

National Geophysical Data Center, 1988: Digital relief of the surface of the Earth (ETOP05). Data Announcement 88-MGG-02, U.S. Dept. of Commerce, Washington, D.C.

NMC Development Division, 1988: Documentation of the research version of the NMC Medium-Range Forecasting model. NMC Development Division, National Meteorological Center, Camp Springs, MD, 504 pp.

Noda, A. and T. Tokioka, 1989: The effect of doubling the CO2 concentration on convective and non-convective precipitation in a general circulation model coupled with a simple mixed layer ocean model. J. Meteor. Soc. Japan, 67, 1057-1069.

Numerical Prediction Division, 1993: Outline of Operational Numerical Weather Prediction at Japan Meteorological Agency. Japan Meteorological Agency, Tokyo, $128 \mathrm{pp}$.

Oh, J.-H., 1989: Physically-based general circulation model parameterization of clouds and their radiative interaction. Ph. D. Dissertation, Department of Atmospheric Sciences, Oregon State University, Corvallis, OR, 315 pp. 
Oh, J.-H., and J.-H. Jung, 1993: Radiative transfer model for climate studies: Solar radiation parameterizations and validation. J. Meteor. Soc. Japan (in press).

Oh, J.-H., and M.E. Schlesinger, 1990: Improvement of the sea-level pressure (SLP) simulation by the University of Illinois two- and multilayer atmospheric general circulation model with various surface wind parameterizations. Internal Report, Department of Atmospheric Sciences, University of Illinois at Urbana.

Oh, J.-H., and M.E. Schlesinger, 1991a: The University of Illinois multilayer atmospheric general circulation model. I. Basic model formulation and performance. Internal Report, Department of Atmospheric Sciences, University of Illinois at Urbana.

Oh, J.-H., and M.E. Schlesinger, 1991b: The University of Illinois multilayer atmospheric general circulation model. II. Cloud parameterization and performance. Internal Report, Department of Atmospheric Sciences, University of Illinois at Urbana.

Oh, J.-H., and M.E. Schlesinger, 1991c: The University of Illinois multilayer atmospheric general circulation model. III. Radiation parameterization and performance. Internal Report, Department of Atmospheric Sciences, University of Illinois at Urbana.

Palmer, T.N., G.J. Shutts, R. Swinbank, 1986: Alleviation of a systematic westerly bias in general circulation and numerical weather prediction models through an orographic gravity wave drag parameterization. Quart. J. Roy. Meteor. Soc., 112, 1001-1039.

Paltridge, G.W., and C.M.R. Platt, 1976: Radiative Processes in Meteorology and Climatology. Elsevier Press, Amsterdam, $318 \mathrm{pp}$.

Pan, H.-L., 1990: A simple parameterization scheme of evapotranspiration over land for the NMC Medium-Range Forecast model. Mon. Wea. Rev., 118, 2500-2512.

Panofsky, H.A., 1973: Tower micrometeorology. In Workshop on Micrometeorology, D.A. Haugen (ed.), American Meteorology Society, Boston, MA, 392 pp.

Parkinson, C.L., and W.M. Washington, 1979: A large scale numerical model of sea ice. J. Geophys. Res., 84, 311-337.

Payne, R.E., 1972: Albedo of the sea surface. J. Atmos. Sci., 29, 959-970. 
Pellerin, G., and R. Benoit, 1987: Champs geophysiques de surface, CMC, NWPM 14 [available from the Canadian Meteorological Centre, Dorval, Quebec].

Peng, L., M.-D. Chou, and A. Arking, 1982: Climate studies with a multi-layer energy balance model, Part I: Model description and sensitivity to solar constant. J. Atmos. Sci., 2639-2656.

Penman, H.L., 1948: Natural evaporation from open water, bare soil, and grass. Proc. Roy. Soc. London, A193, 120-145.

Pepper, D.W., and J. Baker, 1979: A simple one-dimensional finite-element algorithm with multidimensional capability. Num. Heat. Trnsfr., 2, 81-95.

Petzold, D.E., 1977: An estimation technique for snow surface albedo. Climatolog. Bull., 26, 1-11.

Phillips, N.A., 1974: National Meteorology Center Official Note 104. National Weather Service, Washington, D.C., $40 \mathrm{pp}$.

Pierrehumbert, R.T., 1987: An essay on the parameterization of orographic wave drag. In Observation, Theory, and Modelling of Orographic Effects, Vol. 1. European Centre for Medium-Range Weather Forecasts, Reading, England, 251-282.

Pinker, R. T., and I. Laszlo, 1992: Modeling surface solar irradiance for application on a global scale. J. Appl. Meteor., 31, 194-211.

Pitcher, E.J., R.C. Malone, V. Ramanathan, M.L. Blackmon, K. Puri, and W. Bourke, 1983: January and July simulations with a spectral general circulation model. $J$. Atmos. Sci., 40, 580-604.

Platt, C.M.R., and Harshvardhan, 1988: Temperature dependence of cirrus extinction: Implications for climate feedback. J. Geophys. Res., 93, 11051-11058.

Podolskaya, E.L., and I.G. Rivin, 1988: The correction of the integral transmitting function in the longwave part of the spectrum. Izvestia AN SSSR, FAO, 24, 883886 (in Russian).

Pollard, D., M.L. Batteen, and Y.-J. Han, 1983: Development of a simple upper-ocean and sea-ice model. J. Phys. Oceanog., 13, 754-768.

Pollard, D., and S.L. Thompson, 1992: Users' guide to the GENESIS Global Climate Model Version 1.02. Interdisciplinary Climate Systems Section, National Center for Atmospheric Research, Boulder, Colorado, $58 \mathrm{pp}$. 
Pollard, D., and S.L. Thompson, 1994: Use of a land-surface-transfer scheme (LSX) in a global climate model: The response to doubling stomatal resistance. Glob. Plan. Change (submitted).

Posey, T.W., and P.F. Clapp, 1964: Global distribution of normal surface albedo. Geofis. Int., 4, 33-48.

Preuss, J.H., and J.-F. Geleyn, 1980: Surface albedos derived from satellite data and their impact on forecast models. Arch. Met. Geoph. Bioclim., Ser. A, 29, 345-356.

Priestly, C.H.B., 1959: Turbulent Transfer in the Lower Atmosphere. University of Chicago Press, Chicago, IL, 130 pp.

Ramanathan, V., B.R. Barkstrom, and E.F. Harrison, 1989: Climate and the Earth's radiation budget. Physics Today, 42, 22-32.

Ramanathan, V., and R.E. Dickinson, 1979: The role of stratospheric ozone in the zonal and seasonal radiative energy balance of the Earth-troposphere system. $J$. Atmos. Sci., 36, 1084-1104.

Ramanathan, V., and P. Downey, 1986: A nonisothermal emissivity and absorptivity formulation for water vapor. J. Geophys. Res., 91, 8649-8666.

Ramanathan, V., E.J. Pitcher, R.C. Malone, and M. Blackmon, 1983: The response of a general circulation model to refinements in radiative processes. J. Atmos. Sci., 40, 605-630.

Ramanathan, V., H.B. Singh, R.J. Cicerone, and J.T. Kiehl, 1985: Trace gas trends and their potential role in climate change. J. Geophys. Res., 90, 5547-5566.

Ramaswamy, V., and V. Ramanathan, 1989: Solar absorption by cirrus clouds and the maintenance of the tropical upper troposphere thermal structure. J. Atmos. Sci., 46, 2293-2310.

Randall, D., 1976: The interaction of the planetary boundary layer with large-scale circulations. Ph.D. Dissertation, University of California, Los Angeles, $247 \mathrm{pp}$.

Randall, D.A., 1980: Conditional instability of the first kind upside-down. J. Atmos. Sci., 37, 125-130.

Randall, D.A., 1987: Turbulent fluxes of liquid water and buoyancy in partly cloudy layers. J. Atmos. Sci., 44, 850-858. 
Randall, D.A., 1989: A description of the CSU atmospheric general circulation model. Internal Report, Atmospheric Sciences Department, Colorado State University, Fort Collins, CO, 55 pp.

Randall, D.A., J.A. Abeles, and T.G. Corsetti, 1985: Seasonal simulations of the planetary boundary layer and boundary-layer stratocumulus clouds with a general circulation model. J. Atmos. Sci., 42, 641-676.

Randall, D.A., Harshvardhan, T.G. Corsetti, and D.A. Dazlich, 1989: Interactions among clouds, radiation, and convection in a general circulation model. J. Atmos. Sci., 46, 1943-1970.

Randall, D.A., Harshvardhan, and D.A. Dazlich, 1990: Diurnal variability of the hydrological cycle in a general circulation model. J. Atmos. Sci., 48, 40-62.

Rasch, P.J., and D.L. Williamson, 1990: Computational aspects of moisture transport in global models of the atmosphere. Quart.J. Roy. Meteor. Soc., 116, 1071-1090.

Raschke, E., 1973: Approximation of a band of transmission functions by finite sums of exponentials. Contrib. Atmos. Physics, 46, 203-212.

Rikus, L., 1991: The role of clouds in global climate modelling. BMRC Report No. 25, Bureau of Meteorology Research Centre, Melbourne, Australia, 37 pp.

Ritchie, H., 1985: Application of a semi-Lagrangian integration scheme to the moisture equation in a regional forecast model. Mon. Wea. Rev., 113, 424-435.

Ritchie, H., 1986: Eliminating the interpolation associated with the semi-Lagrangian scheme. Mon. Wea. Rev., 114, 135-146.

Ritchie, H., 1987: Semi-Lagrangian advection on a Gaussian grid. Mon. Wea. Rev., 15, 608-619.

Ritchie, H., 1988: Application of the semi-Lagrangian method to a spectral model of the shallow water equations. Mon. Wea. Rev., 116, 1587-1598.

Ritchie, H., 1991: Application of the semi-Lagrangian method to a multilevel spectral primitive-equations model. Quart.J. Roy. Meteor. Soc., 117, 91-106.

Ritter, B., and J.-F. Geleyn, 1992: A comprehensive radiation scheme of numerical weather prediction with potential application to climate simulations. Mon. Wea. Rev., 120, 303-325. 
Robert, A.J., 1966: The integration of a low order spectral form of the primitive meteorological equations. J. Meteor. Soc. Japan, 44, 237-245.

Robert, A.J., 1981: A stable numerical integration scheme for the primitive meteorological equations. Atmos.-Ocean, 19, 35-46.

Robert, A.J., 1982: A semi-Lagrangian and semi-implicit numerical integration scheme for the primitive meteorological equations. J. Meteor. Soc. Japan, 60, 319-325.

Robert, A.J., J. Henderson, and C. Turnbull, 1972: An implicit time integration scheme for baroclinic models in the atmosphere. Mon. Wea. Rev., 100, 329-335.

Roberts, R.E., J.A. Selby, and L.M. Biberman, 1976: Infrared continuum absorption by atmospheric water vapor in the 8-12 micron window. Appl. Optics, 15, 20852090.

Robock, A., 1980: The seasonal cycle of snow cover, sea-ice and surface albedo. Mon. Wea. Rev., 108, 267-285.

Rockel, B., E. Raschke, and B. Weyres, 1991: A parameterization of broad band radiative transfer properties of water, ice and mixed clouds. Beitr. Phys. Atmos., 64, $1-12$.

Rodgers, C.D., 1967a: The radiative heat budget of the troposphere and lower stratosphere. Report No. 2, Department of Meteorology, Massachusetts Institute of Technology, Cambridge, MA, 99 pp.

Rodgers, C.D., 1967b: The use of emissivity in atmospheric radiation calculations. Quart. J. Roy. Meteor. Soc., 93,43-54.

Rodgers, C.D., 1968: Some extensions and applications of the new random model for molecular band transmission. Quart. J. Roy. Meteor. Soc., 94, 99-102.

Rodgers, C.D., and C.D. Walshaw, 1966: The computation of infra-red cooling rate in planetary atmospheres. Quart. J. Roy. Meteor. Soc., 92, 67-92.

Roeckner, E., K. Arpe, L. Bengtsson, S. Brinkop, L. Duemenil, M. Esch, E. Kirk, F. Lunkeit, M. Ponater, B. Rockel, R. Sausen, U. Schlese, S. Schubert, and M. Windelband, 1992: Simulation of the present-day climate with the ECHAM model: Impact of model physics and resolution. MPI Report No. 93, ISSN 0937-1060, Max-Planck-Institut fuer Meteorologie, Hamburg, Germany, $171 \mathrm{pp}$. 
Roeckner, E., M. Rieland, and E. Keup, 1991: Modelling of cloud and radiation in the ECHAM model. Proceedings of the ECMWF/WCRP Workshop on Clouds, Radiative Transfer and the Hydrological Cycle, 12-15 Nov. 1990, European Centre for Medium-Range Weather Forecasts, Reading, England, 199-222.

Roeckner, E., and U. Schlese, 1985: January simulation of clouds with a prognostic cloud cover scheme. Proceedings of the ECMWF Workshop on Cloud Cover in Numerical Models, 26-28 Nov. 1984, European Centre for Medium-Range Weather Forecasts, Reading, England, 87-108.

Rood, R.B., 1987: Numerical advection algorithms and their role in atmospheric transport and chemistry models. Rev. Geophys., 25, 71-100.

Rosenfield, J.E., M.R. Schoeberl, and M.A. Geller, 1987: A computation of the stratospheric diabatic circulation using an accurate radiative transfer model. $J$. Atmos. Sci., 44, 859-876.

Rothman, L.S., 1981: AFGL atmospheric absorption line parameters compilation: 1980 version. Appl. Optics, 20, 791-795.

Rothman, L.S., R.R. Gamache, A. Barbe, A. Goldman, J.R. Gillis, L.R. Brown, R.A. Toth, J.-M. Flaud, and C. Camy-Peyret, 1983: AFGL atmospheric absorption line parameters compilation: 1982 edition. Appl. Optics, 22, 2247-2256.

Royer, J.-F., 1986: Correction of negative mixing ratios in spectral models by global horizontal borrowing. Mon. Wea. Rev., 114, 1406-1410.

Royer, J.-F., D. Cariolle, and J.P. Rocafort, 1988: Influence of vertical resolution and ozone distribution on the systematic errors of the French spectral GCM. In Proceedings of the WMO Workshop on Systematic Errors in Models of the Atmosphere, Toronto. [Available from the World Meteorological Organization, Geneva.]

Royer, J.-F., S. Planton, and M. Deque, 1990: A sensitivity experiment for the removal of Arctic sea ice with the French spectral general circulation model. Clim. Dynam.., 5, 1-17.

Rozanov, E.V., and V.A. Frolkis, 1988: Method of radiation fluxes calculation in the near infrared range. Trudy GGO (MGO Proc.), No. 516, $61-71$ (in Russian).

Ruprecht, E., and W.M. Gray, 1976: Analysis of satellite-observed tropical cloud clusters, II: Thermal, moisture, and precipitation fields. Tellus, 28, 414-426. 
Russell, G., and J. Lerner, 1981: A new finite-differencing scheme for the tracer transport equation. J. Appl. Meteor., 20, 1483-1498.

Sadourny, R., 1975a: The dynamics of finite difference models of the shallow water equations. J. Atmos. Sci., 32, 680-689.

Sadourny, R., 1975b: Compressible model flows on the sphere. J. Atmos. Sci., 32, 2103-2110.

Sadourny, R., 1980: Conservation laws, turbulence, and numerical modelling of largescale flow. Proceedings of the 1979 ECMWF Seminar, European Centre for Medium-Range Weather Forecasts, Reading, England, 167-195.

Sadourny, R., and K. Laval, 1984: January and July performance of the LMD general circulation model. In New Perspectives in Climate Modeling, A.L. Berger and C. Nicolis (eds.), Elsevier Press, Amsterdam, 173-197.

Sagan, C., and J.B. Pollack, 1967: Anisotropic nonconservative scattering and the clouds of Venus. J. Geophys. Res., 72, 466-477.

Saito, K., and A. Baba, 1988: A statistical relation between relative humidity and the GMS observed cloud amount. J. Meteor. Soc. Japan, 66, 187-192.

Sasamori, T., 1959: The temperature effect of the absorption of the 15-micron carbondioxide band. Science Reports of the Tohoku University, Geophysics Ser. 5, Vol. $11,149-161$.

Sasamori, T., 1972: A linear harmonic analysis of atmospheric motion with radiative dissipation. J. Meteor. Soc. Japan, 50, 505-518.

Sasamori, T., J. London, and D.V. Hoyt, 1972: Radiation budget of the Southern Hemisphere. Meteorological Monographs, 35, American Meteorological Society, Boston, MA, 9-22.

Sassen, K., and G.C. Dodd, 1989: Haze particle nucleation simulations in cirrus cloud and applications for numerical and lidar studies. J. Atmos. Sci., 46, 3005-3014.

Sato, N., P.J. Sellers, D.A. Randall, E.K. Schneider, J. Shukla, J.L. Kinter III, Y-T. Hou, and E. Albertazzi, 1989a: Implementing the simple biosphere model in a general circulation model: Methodologies and results. NASA Contractor Report 185509, Center for Land-Ocean-Atmosphere Interactions, University of Maryland at College Park, 76 pp. 
Sato, N., P.J. Sellers, D.A. Randall, E.K. Schneider, J. Shukla, J.L. Kinter III, Y.-T. Hou, and E. Albertazzi, 1989b: Implementing the simple biosphere model in a general circulation model. J. Atmos. Sci., 46, 2757-2782.

Schemm, J., S. Schubert, J. Terry, and S. Bloom, 1992: Estimates of monthly mean soil moisture for 1979-1989. NASA Tech. Memo. No. 104571, Goddard Space Flight Center, Greenbelt, MD, 252 pp.

Schlesinger, M.E., 1976: A numerical simulation of the general circulation of atmospheric ozone. Ph.D. Dissertation, Dept. of Atmospheric Sciences, University of California, Los Angeles, $376 \mathrm{pp}$.

Schlesinger, M.E., and Y. Mintz, 1979: Numerical simulation of ozone production, transport and distribution with a global atmospheric general circulation model. J. Atmos. Sci., 36, 1325-1361.

Schlesinger, M.E., J.-H. Oh, and D. Rosenfeld, 1988: A parameterization of the evaporation of rainfall. Mon. Wea. Rev., 116, 1887-1895.

Schneider, E.K., and R.S. Lindzen, 1976: A discussion of the parameterization of momentum exchange of cumulus convection. J. Geophys. Res., 81, 3158-3160.

Schubert, S.D., R.B. Rood, and J. Pfaendtner, 1993: An assimilated dataset for Earth science applications. Bull. Amer. Meteor. Soc., 74, 2331-2342.

Schuman, F.G., 1971: Resuscitation of an integration procedure. NMC Office Note, National Meteorological Center, Washington, D.C.

Schwarzkopf, M.D., and S.B. Fels, 1985: Improvements to the algorithm for computing $\mathrm{CO}_{2}$ transmissivities and cooling rates. J. Geophys. Res., 90, 10541-10550.

Schwarzkopf, M.D., and S.B. Fels, 1991: The simplified exchange method revisited: An accurate, rapid method for computation of infrared cooling rates and fluxes. J. Geophys. Res., 96, 9075-9096.

Sela, J., 1980: Spectral modeling at the National Meteorological Center. Mon. Wea. Rev., 108, 1279-1292.

Sela, J., 1982: The NMC spectral model. NOAA Tech. Report NWS 30, National Weather Service, National Oceanic and Atmospheric Administration, U.S. Dept. of Commerce, Washington, D.C., $36 \mathrm{pp}$. 
Sellers, P.J., Y. Mintz, Y.C. Sud, and A. Dalcher, 1986: A simple biosphere model (SiB) for use within general circulation models. J. Atmos. Sci., 43, 505-531.

Semtner, A.J., 1976: A model for the thermodynamic growth of sea ice in numerical investigations of climate. J. Phys. Oceanog., 6, 379-389.

Shapiro, R., 1970: Smoothing, filtering and boundary effects. Rev. Geophys. Space Phys., 8, 359-387.

Sheinin, D.A., 1983: Construction of a semi-implicit dissipative time integration scheme with a negligible computational mode. Trudy GGO (MGO Proc.), No. 481, 108-128 (in Russian).

Sheinin, D.A., 1987: Thermodynamically consistent schemes for vertical approximation of the atmospheric dynamics equations. Meteorologia i Hydrologia, 6, 34-44 (in Russian).

Shettle, E.P., and R. Fenn, 1975: Models of the atmospheric aerosols and their optical properties. Proceedings of the AGARD Conference, No. 183, AGARD-CP-183.

Shibata, K., and T. Aoki, 1989: An infrared radiative scheme for the numerical models of weather and climate. J. Geophys. Res., 94, 14923-14943.

Shuttleworth, W.J., 1988: Macrohydrology: The new challenge for process hydrology. J. Hydrol., 100, 31-56.

Simmons, A.J., and D.M. Burridge, 1981: An energy and angular-momentum conserving vertical finite difference scheme and hybrid vertical coordinates. Mon. Wea. Rev., 109, 758-766.

Simmons, A.J., D.M. Burridge, M. Jarraud, C. Girard, and W. Wergen, 1989: The ECMWF medium-range prediction models: Development of the numerical formulations and the impact of increased resolution. Meteor. Atmos. Phys., 40, 28-60.

Simmons, A.J., B.J. Hoskins, and D.M. Burridge, 1978: Stability of the semi-implicit method of time integration. Mon. Wea. Rev., 106, 405-412.

Simmons, A.J., and M. Jarraud, 1983: The design and performance of the new ECMWF operational model. Proceedings of the ECMWF Workshop on Numerical Methods for Weather Prediction, European Centre for MediumRange Weather Forecasts, Reading, England, 113-164. 
Simmons, A.J., and R. Struefing, 1981: An energy and angular-momentum conserving finite difference scheme, hybrid coordinates and medium-range weather prediction. ECMWF Tech. Report No. 28, European Centre for Medium-Range Weather Forecasts, Reading, England, 68 pp.

Simpson, J., and V. Wiggard, 1969: Models of precipitating cumulus towers, Mon. Wea. Rev., 97, 471-489.

Slingo, A. (ed.), 1985: Handbook of the Meteorological Office 11-layer atmospheric general circulation model. Vol. 1: Model description. DCTN 29, United Kingdom Meteorological Office, Bracknell, Berkshire RG12 2SZ, UK.

Slingo, A., 1989: A GCM parameterization for the shortwave radiative properties of water clouds. J. Atmos. Sci., 46, 1419-1427.

Slingo, A., and H.M. Schrecker, 1982: On the shortwave radiative properties of stratiform water clouds. Quart. J. Roy. Meteor. Soc., 108, 407-426.

Slingo, A., and J.M. Slingo, 1991: Response of the National Center for Atmospheric Research Community Climate Model to improvements in the representation of clouds. J. Geophys. Res., 96, 15341-15357.

Slingo, A., and R.C. Wilderspin, 1986: Development of a revised longwave radiation scheme for an atmospheric general circulation model. Quart. J. Roy. Meteor. Soc., 112, 371-386.

Slingo, J.M., 1987: The development and verification of a cloud prediction scheme for the ECMWF model. Quart. J. Roy. Meteor. Soc., 113, 899-927.

Slingo, J.M., M. Blackburn, A.K. Betts, R. Brugge, B.J. Hoskins, M.J. Miller, L. Steenman-Clark, and J. Thuburn, 1994: Mean climate and transience in the tropics of the UGAMP GCM. Part I: Sensitivity to convective parameterization. Quart. J. Roy. Meteor. Soc. (in press).

Slingo, J.M., and B. Ritter, 1985: Cloud prediction in the ECMWF model. ECMWF Tech. Report No. 46, European Centre for Medium-Range Weather Forecasts, Reading, England, 48 pp.

Smagorinsky, J., 1960: On the dynamical prediction of large-scale condensation by numerical methods. Geophysical Monographs, 5, American Geophysical Union, Washington, D.C., 71-78. 
Smagorinsky, J., 1963: General circulation experiments with the primitive equations. I. The basic experiment. Mon. Wea. Rev., 91, 99-164.

Smagorinsky, J., S. Manabe, and J.L. Holloway, Jr., 1965: Results from a nine-level general circulation model of the atmosphere. Mon. Wea. Rev., 93, 727-768.

Smith, G.D., 1965: Numerical Solution of Partial Differential Equations. Oxford University Press, London, $179 \mathrm{pp}$.

Smith, R.N.B., 1990a: A scheme for predicting layer clouds and their water content in a general circulation model. Quart. J. Roy. Meteor. Soc., 116, 435-460.

Smith, R.N.B., 1990b: Subsurface, surface, and boundary layer processes. Unified Model Documentation Paper No. 24, United Kingdom Meteorological Office, Bracknell, Berkshire RG12 2SZ, UK.

Smith, R.N.B., 1993: Experience and developments with the layer cloud and boundary layer mixing schemes in the UK Meteorological Office Unified Model. In Proceedings of the ECMWF/GCSS Workshop on Parameterisation of the CloudTopped Boundary Layer, 8-11 June 1993, European Centre for Medium-Range Weather Forecasts, Reading, England.

Smith, R.N.B., and D. Gregory, 1990: Large-scale precipitation. Unified Model Documentation Paper No. 26, United Kingdom Meteorological Office, Bracknell, Berkshire RG12 2SZ, UK.

Smith, S.D., 1980: Wind stress and heat flux over the ocean in gale force winds. $J$. Phys. Oceanog., 10, 709-726.

Sokolov, A.P., 1986: Simulation of the January atmospheric circulation using a global spectral model. Meteorologia i Hydrologia, 6, 12-21 (in Russian).

Sokolov, A.P., and D.A. Sheinin, 1983: Non-diabatic version of the baroclinic spectral atmospheric model. Trudy GGO (MGO Proc.), No. 481, 93-102 (in Russian).

Somerville, R.C.J., P.H. Stone, M. Halem, J.E. Hansen, J.S. Hogan, L.M. Druyan, G. Russell, A.A. Lacis, W.J. Quirk, and J. Tennenbaum, 1974: The GISS model of the global atmosphere. J. Atmos. Sci., 31, 84-117.

Spivakovsky, C.M., R. Yevich, J.A. Logan, S.C. Wofsy, and M.B. McElroy, 1990: Tropospheric $\mathrm{OH}$ in a three-dimensional chemical tracer model: An assessment based on observations of $\mathrm{CH}_{3} \mathrm{CCl}_{3}$. J. Geophys. Res., 95, 18441-18471. 
Squires, P., 1958: The microstructure and colloidal stability of warm clouds, Part I: The relation between structure and stability. Tellus, 10, 256-262.

Staley, D.O., and G.M. Jurica, 1972: Effective atmospheric emissivity under clear skies. J. Appl. Meteor., 11, 349-356.

Starr, D. O'C., and S.K. Cox, 1985: Cirrus clouds, Part II: Numerical experiments on the formation and maintenance of cirrus. J. Atmos. Sci., 42, 2682-2694.

Stephens, G.L., 1978a: Radiation profiles in extended water clouds. I: Theory. J. Atmos. Sci., 35, 2111-2122.

Stephens, G.L., 1978b: Radiation profiles in extended water clouds. II: Parameterization schemes. J. Atmos. Sci., 35, 2123-2132.

Stephens, G.L., 1979: Optical properties of eight water cloud types. CSIRO, Division of Atmospheric Physics, Tech. Paper No. 36, 35 pp. [Available from Commonwealth Scientific and Industrial Research Organization, Mordialloc, Victoria 3195, Australia.]

Stephens, G.L., D.A. Randall, I.L. Wittmeyer, and D.A. Dazlich, 1993: The earth's radiation budget and its relation to atmospheric hydrology 3. Comparison of observations over the oceans with a GCM. J. Geophys. Res., 98, 4931-4950.

Stern, W.F., and R.T. Pierrehumbert, 1988: The impact of an orographic gravity wave drag parameterization on extended range predictions with a GCM. Preprints of the Eighth Conference on Numerical Weather Prediction, American Meteorological Society, Baltimore, 745-750.

Stolarski, R.S., P. Bloomfield, R.D. McPeters, and J.R. Herman, 1991: Total ozone trends deduced from Nimbus-7 TOMS data. Geophys. Res. Letters, 18, 10151018.

Stone, H.M., and S. Manabe, 1968: Comparison among various numerical models designed for computing infrared cooling. Mon. Wea. Rev., 96, 735-741.

Suarez, M. J., A. Arakawa, and D.A. Randall, 1983: Parameterization of the planetary boundary layer in the UCLA general circulation model: Formulation and results. Mon. Wea. Rev., 111, 2224-2243.

Suarez, M.J., and L.L. Takacs, 1993: Documentation of the ARIES/GEOS dynamical core Version 2. NASA Tech. Memo., Goddard Space Flight Center, Greenbelt, MD. [Available from M.J. Suarez, GSFC, Code 913, Greenbelt, MD 20771.] 
Sud, Y.C., W.C. Chao, and G.K. Walker, 1991: Contributions to the implementation of Arakawa-Schubert cumulus parameterizations in the GLA GCM. J. Atmos. Sci., 48, 1573-1586.

Sud, Y.C., W.C. Chao, and G.K. Walker, 1992: Role of a cumulus parameterization scheme in maintaining atmospheric circulation and rainfall in the nine-layer Goddard Laboratory for Atmospheres general circulation model. Mon. Wea. Rev., 120, 594-611.

Sud, Y.C., and A. Molod, 1986: Rain-evaporation parameterization for the GLA GCM. GMSB/NASA Research Review--1985, NASA Goddard Space Flight Center, Greenbelt, MD.

Sud, Y.C., and A. Molod, 1988: The roles of dry convection, cloud-radiation feedback processes and the influence of recent improvements in the parameterization of convection in the GLA GCM. Mon. Wea. Rev., 116, 2366-2387.

Sud, Y.C., and G.K. Walker, 1992: A review of recent research on improvement of physical parameterizations in the GLA GCM. In Physical Processes in Atmospheric Models, D.R. Sikka and S.S. Singh (eds.), Wiley Eastern Ltd., New Delhi, $422-479$.

Sud, Y.C., and G.K. Walker, 1993: A rain-evaporation and downdraft parameterization to complement a cumulus updraft scheme and its evaluation using GATE data. Mon. Wea. Rev., 11, 3019-3039.

Sugi, M., K. Kuma, K. Tada, K. Tamiya, N. Hasegawa, T. Iwasaki, S. Yamada, and T. Kitade, 1989: Description and performance of the JMA operational global spectral model (JMA-GSM88). JMA/NPD Tech. Report No. 27, Japan Meteorological Agency, Tokyo, 48 pp.

Sundqvist, H., 1978: A parameterization scheme for non-convective condensation including prediction of cloud water content. Quart. J. Roy. Meteor. Soc., 104,677690 .

Sundqvist, H., 1981: Prediction of stratiform clouds: Results from a 5-day forecast with a global model. Tellus, 33, 242-253.

Sundqvist, H., 1988: Parameterization of condensation and associated clouds in models for weather prediction and general circulation simulation. In PhysicallyBased Modelling and Simulation of Climate and Climatic Change, Part 1. M.E. Schlesinger (ed.), Kluwer Academic Publishers, Dordrecht, 433-462. 
Takano, K., and M.G. Wurtele, 1982: A fourth-order energy and potential enstrophy conserving difference scheme. Air Force Geophysics Laboratory Report, AFGLTR-82-0205, Hanscom Air Force Base, Bedford, MA, 85 pp.

Tanré, D., J.F. Geleyn, and J. Slingo, 1983: First results of the introduction of an advanced aerosol-radiation interaction in the ECMWF low resolution model. Proceedings of the WMO/IAMAP Meeting of Experts on Aerosols and Their Climate Effects, A. Deepak and H.E. Gerber (eds.), Hampton, VA, 133-177.

Thomas, G., and A. Henderson-Sellers, 1991: An evaluation of proposed representations of subgrid hydrologic processes in climate models. J. Climate, 4, 898-910.

Thompson, S.L., and D. Pollard, 1994: A global climate model (GENESIS) with a landsurface-transfer scheme (LSX). Part 1: Present climate simulation. J. Climate (submitted).

Thompson, S.L., V. Ramaswamy, and C. Covey, 1987: Atmospheric effects of nuclear war aerosols in general circulation model simulations: Influence of smoke optical properties. J. Geophys. Res., 92, 10942-10960.

Thuburn, J., 1993: Use of a flux-limited scheme for vertical advection in a GCM. Quart. J. Roy. Meteor. Soc., 119, 469-487.

Tibaldi, S., and J.-F. Geleyn, 1981: The production of a new orography, land-sea mask and associated climatological surface fields for operational purposes. ECMWF Tech. Memo. No. 40, European Centre for Medium-Range Weather Forecasts, Reading, England, 93 pp.

Tiedtke, M., 1983: The sensitivity of the time-mean large-scale flow to cumulus convection in the ECMWF model. Proceedings of the ECMWF Workshop on Convection in Large-Scale Models, 28 November-1 December 1983, European Centre for Medium-Range Weather Forecasts, Reading, England, 297-316.

Tiedtke, M., 1984: The effect of penetrative cumulus convection on the large-scale flow in a general circulation model. Beitr. Phys. Atmos., 57, 216-239.

Tiedtke, M., 1988: Parameterization of cumulus convection in large-scale models. In Physically-Based Modelling and Simulation of Climate and Climatic Change, Part 1. M.E. Schlesinger (ed.), Kluwer Academic Publishers, Dordrecht, 375-431.

Tiedtke, M., 1989: A comprehensive mass flux scheme for cumulus parameterization in large-scale models. Mon. Wea. Rev., 117, 1779-1800. 
Tiedtke, M., W.A. Heckley, and J. Slingo, 1988: Tropical forecasting at ECMWF: The influence of physical parameterization on the mean structure of forecasts and analyses. Quart. J. Roy. Meteor. Soc., 114, 639-664.

Tokioka, T., 1978: Some considerations on vertical differencing. J. Meteor. Soc. Japan, 56, 98-111.

Tokioka, T., K. Yamazaki, A. Kitoh, and T. Ose, 1988: The equatorial 30-60 day oscillation and the Arakawa-Schubert penetrative cumulus parameterization. $J$. Meteor. Soc. Japan, 66, 883-901.

Tokioka, T., K. Yamazaki, I. Yagai, and A. Kitoh, 1984: A description of the Meteorological Research Institute atmospheric general circulation model (MRI GCM-I). MRI Tech. Report No. 13, Meteorological Research Institute, Ibaraki-ken, Japan, 249 pp.

Toon, O.B., and J.B. Pollack, 1976: A global average model of atmospheric aerosols for radiative transfer and calculations. J. Appl. Meteor., 15, 225-246.

Troen, I., and L. Mahrt, 1986: A simple model of the atmospheric boundary layer: Sensitivity to surface evaporation. Bound. Layer Meteor., 37, 129-148.

U.S. Army Topographic Command and the U.S. Naval Oceanographic Office, 1971: The World. Series 1142 (1st edition), Washington, D.C.

van den Dool, H. M., and S. Saha, 1993: Seasonal redistribution and conservation of atmospheric mass in a general circulation model. J. Climate, 6, 22-30.

Vinnikov, K.Ya, and I.B. Yeserkepova, 1991: Soil moisture: Empirical data and model results. J. Climate, 4, 66-79.

Wallace, J., S. Tibaldi, and A.J. Simmons, 1983: Reduction of systematic forecast errors in the ECMWF model through the introduction of an envelope orography. Quart. J. Roy. Meteor. Soc., 109, 683-717.

Walshaw, C.D., 1957: Integrated absorption by the 9.6 micron band of ozone. Quart. J. Roy. Meteor. Soc., 83, 315-321.

Wang, W.-C., M.P. Dudek, X.-Z. Liang, and J.T. Kiehl, 1991a: Inadequacy of effective $\mathrm{CO}_{2}$ as a proxy in simulating the greenhouse effect of other radiatively active gases. Nature, 350, 573-577. 
Wang, W.-C., G-Y. Shi, and J.T. Kiehl, 1991b: Incorporation of the thermal radiative effect of $\mathrm{CH}_{4}, \mathrm{~N}_{2} \mathrm{O}, \mathrm{CF}_{2} \mathrm{Cl}_{2}$, and $\mathrm{CFCl}_{3}$ into the NCAR Community Climate Model. J. Geophys. Res., 96, 9097-9103.

Wang, W.-C., X.-Z. Liang, M.P. Dudek, D. Pollard, and S.L. Thompson, 1994: Atmospheric ozone as a climate gas. Atmos. Res. (submitted).

Warrilow, D.A., A.B. Sangster, and A. Slingo, 1986: Modelling of land surface processes and their influence on European climate. DCTN 38, Dynamical Climatology Branch, United Kingdom Meteorological Office, Bracknell, Berkshire RG12 2SZ, UK.

Warrilow, D.A., R.N.B. Smith, and F.E. Hewer, 1990: Snow processes in the 5th annual cycle integration. Met. Office Internal Note No. 93. United Kingdom Meteorological Office, Bracknell, Berkshire RG12 2SZ, UK.

Washington, W.M. (ed.), 1982: Documentation for the Community Climate Model (CCM) Version 0. NCAR Climate Section Publication, National Center for Atmospheric Research, Boulder, CO.

Washington, W.M., and G.A. Meehl, 1984: A seasonal cycle experiment on the climate sensitivity due to a doubling of $\mathrm{CO}_{2}$ with an atmospheric general circulation model coupled to a simple mixed layer ocean model. J. Geophys. Res., 89, 94759503.

Washington, W.M., and G.A. Meehl, 1986: $\mathrm{GCM} \mathrm{CO}_{2}$ sensitivity experiments: Snow sea ice albedo parameterizations and globally averaged surface air temperature. Clim. Change, 8, 231-241.

Washington, W.M., and L. Verplank, 1986: A description of coupled general circulation models of the atmosphere and oceans used for carbon dioxide studies. NCAR Tech. Note, NCAR/TN-271+EDD, National Center for Atmospheric Research, Boulder, CO, 29 pp.

Washington, W.M., and D.L. Williamson, 1977: A description of the NCAR GCM. In Methods in Computational Physics, 17, J. Chang (ed.), Academic Press, New York, 111-172.

Webb, R.S., C.E. Rosenzweig, and E.R. Levine, 1993: Specifying land surface characteristics in general circulation models: Soil profile data set and derived waterholding capacities. Glob. Biogeochem. Cycles, 7, 97-108. 
Wetherald, R.T., and S. Manabe, 1988: Cloud feedback processes in a general circulation model. J. Atmos. Sci., 45, 1397-1415.

Wetherald, R.T., V. Ramaswamy, and S. Manabe, 1991: A comparative study of the observations of high clouds and simulations by an atmospheric general circulation model. Clim. Dynam., 5, 135-143.

White, A.A., and R.A. Bromley, 1988: A new set of dynamical equations for use in numerical weather prediction and global climate models. Met. Off. $13 \mathrm{Branch}$ Memo., United Kingdom Meteorological Office, Bracknell, Berkshire RG12 2SZ, UK.

Wilcox, R.W., and A.D. Belmont, 1977: Ozone concentration by latitude, altitude, and month near 80 degrees West. Report No. FAA-AEQ-77-13, FAA Office of Environmental Quality, High Altitude Pollution Program, U.S. Department of Transportation,Washington, D.C., $41 \mathrm{pp}$.

Williamson, D.L., 1983: Description of the NCAR Community Climate Model (CCM0B). NCAR Tech. Note NCAR/TN-210+STR, National Center for Atmospheric Research, Boulder, CO, 88 pp.

Williamson, D.L., 1988: The effect of vertical finite difference approximations on simulations with the NCAR Community Climate Model. J. Climate, 1, 40-58.

Williamson, D.L., L.M. Bath, R.K. Sato, T.A. Mayer, and M.L. Kuhn, 1983: Documentation of NCAR CCM0B program modules. NCAR Tech. Note NCAR/TN-212+IA, National Center for Atmospheric Research, Boulder, CO, 198 pp.

Williamson, D.L., J.T. Kiehl, V. Ramanathan, R.E. Dickinson, and J.J. Hack, 1987: Description of NCAR Community Climate Model (CCM1). NCAR Tech. Note NCAR/TN-285+STR, National Center for Atmospheric Research, Boulder, CO, $112 \mathrm{pp}$.

Williamson, D.L., and P.J. Rasch, 1989: Two-dimensional semi-Lagrangian transport with shape-preserving interpolation. Mon. Wea. Rev., 117, 102-129.

Williamson, D.L., and P.J. Rasch, 1993: Water vapor transport in the NCAR CCM2. Tellus (in press).

Williamson, G.S., 1993: CCM2 datasets and circulation statistics. NCAR Tech. Note NCAR/N-391+STR, National Center for Atmospheric Research, Boulder, CO, 85 pp. 
Willmott, C.J., C. M. Rowe, and Y. Mintz, 1985: Climatology of the terrestrial seasonal water cycle. J. Climatology, 5, 589-606.

Wilson, C.A., 1989: Vertical diffusion. Unified Model Documentation Paper No. 21, United Kingdom Meteorological Office, Bracknell, Berkshire RG12 2SZ, UK.

Wilson, C.A., and J.F.B. Mitchell, 1987: A doubled $\mathrm{CO}_{2}$ climate sensitivity experiment with a global climate model including a simple ocean. J. Geophys. Res., 92, 13315-13343.

Wilson, C.A., and R. Swinbank, 1989: Gravity wave drag. Unified Model Documentation Paper No. 22, United Kingdom Meteorological Office, Bracknell, Berkshire RG12 2SZ, UK.

Wilson, M.F., and A. Henderson-Sellers, 1985: A global archive of land cover and soils data sets for use in general circulation models. J. Climatology, 5, 119-143.

Wiscombe, W.J., and J.W. Evans, 1977: Exponential sum fitting of radiative transmission functions. J. Comput. Phys., 24, 416-444.

Wiscombe, W.J., and S.G. Warren, 1980: A model for the spectral albedo of snow, I: Pure snow. J. Atmos. Sci., 37, 2712-2733.

WMO-ICSU, 1984: Optical properties for the standard aerosols of the Radiation Commission. Report WCP-55, World Climate Program, World Meteorological Organization and International Council of Scientific Unions, Geneva.

Wu, J., 1982: Wind-stress coefficients over sea surface from breeze to hurricane. $J$. Geophys. Res., 87, 9704-9706.

Wurtele, M.G., R.D. Sharman, and T.L. Keller, 1987: Analysis and simulation of a troposphere-stratosphere gravity wave model. Part I. J. Atmos. Sci., 44, 3269-3281.

Xu, K.M., and S.K. Krueger 1991: Evaluation of cloudiness parameterizations using a cumulus ensemble model. Mon. Wea. Rev., 119, 342-367.

Xue, Y.-K., P.J. Sellers, J.L. Kinter II, and J. Shukla, 1991: A simplified biosphere model for global climate studies. J. Climate, 4, 345-364.

Yagai, I., 1986: The effect of clouds on the medium range weather forecasting. Extended abstracts of the WMO/IUGG International Symposium on Short- and MediumRange Numerical Weather Prediction, 4-8 August 1986, Tokyo, Japan, 159-162. 
Yagai, I., and T. Tokioka, 1987: The effect of increased surface drag coefficient over the continents on January circulations. Short-and Medium-Range Numerical Weather Prediction (Special Volume of J. Meteor. Soc. Japan), T. Matsuno (ed.), 409-419.

Yagai, I., and K. Yamazaki, 1988: Effect of the internal gravity wave drag on the 12layer MRI GCM January simulation. Report No. 12 of the Proceedings of the WGNE Workshop on Systematic Errors in Models of the Atmosphere, 19-23 September 1988, Working Group on Numerical Experimentation, Toronto, 8 pp.

Yaglom, A.M., and B.A. Kader, 1974: Heat and mass transfer between a rough wall and turbulent fluid flow at high Reynolds and Peclet numbers. J. Fluid Mech., 62, 601-623.

Yamamoto, G., 1962: Direct absorption of solar radiation by atmospheric water vapor, carbon dioxide, and molecular oxygen. J. Atmos. Sci., 19, 182-188.

Yanai, M., S. Esbensen, and J.-H., Chu, 1973: Determination of bulk properties of tropical cloud clusters from large-scale heat and moisture budgets. J. Atmos. Sci., 30, 611-627.

Yao, M.S., and A.D. Del Genio., 1989: Effects of cumulus entrainment and multiple cloud types on a January global climate model simulation. J. Climate, 2, 850-863.

Zalesak, S.T., 1979: Fully multidimensional flux-corrected transport algorithms for fluids. J. Comput. Phys., 31, 335-362.

Zdunkowski, W.G., W.-G. Panhans, R.M. Welch, and G.J. Korb, 1982: A radiation scheme for circulation and climate models. Contrib. Atmos. Phys., 55, 215-238.

Zdunkowski, W.G., R.M. Welch, and G.J. Korb, 1980: An investigation of the structure of typical two-stream methods for the calculation of solar fluxes and heating rates in clouds. Beitr. Phys. Atmos., 53, 147-166.

Zeng, Q.C., 1979: Physical-Mathematical Basis of Numerical Weather Prediction, Vol. 1. Science Press, Beijing, 543 pp.

Zeng, Q.C., C.G. Yuan, X.H. Zhang, Z.Z. Liang, and N. Bao, 1987: A global gridpoint general circulation model. Proceedings of the WMO Numerical Weather Prediction Symposium, 4-8 August 1986, Tokyo, World Meteorological Organization, 421-430. 
Zeng, Q.C. and X.H. Zhang, 1982: Perfectly energy-conservative time-space finitedifference scheme and the consistent split method to solve the dynamical equations of a compressible fluid. Scientia Sinica, Series B, XXV(8), 866-880.

Zeng, Q.C. and X.H. Zhang, 1987: Available energy conserving schemes for primitive equations of a spherical baroclinic atmosphere. Chinese J. Atmos. Sci., 11(2), 121-142.

Zeng, Q.C., X.H. Zhang, X.Z. Liang, C.G. Yuan, and S.F. Chen, 1989: Documentation of IAP two-level atmospheric general circulation model. DOE/ER/60314-HI, U.S. Department of Energy, Washington, D.C., 383 pp.

Zilitinkevich, S.S., 1970: Dynamics of the Atmospheric Boundary Layer. Gidrometeoizdat, Leningrad, $292 \mathrm{pp}$ (in Russian). 


\section{PCMDI REPORTS}

Number

Title

Author(s)

$\underline{\text { Date }}$

1

The Validation of Atmospheric Models

2

$$
\begin{gathered}
\text { Analysis of the Temporal Behavior } \\
\text { of Tropical Convection in the } \\
\text { ECMWF Model }
\end{gathered}
$$

W. L. Gates

March 1992

J. M. Slingo

April 1992

K. R. Sperber

J.-J. Morcrette

G. L. Potter

3 The Effect of Horizontal Resolution

P. J. Gleckler

on Ocean Surface Heat Fluxes in the ECMWF Model

K. E. Taylor

July 1992

4 Behavior of an Ocean General

C. Covey

August 1992 Circulation Model at Four Different Horizontal Resolutions

5 The Effects of Sampling Frequency on the Climate Statistics of the ECMWF General Circulation Model

T. J. Phillips

September 1992 W. L. Gates

K. Arpe

6 Sensitivity of Dynamical Quantities J. S. Boyle

October 1992 to Horizontal Resolution in a Climate Simulation with the ECMWF Atmospheric General

Circulation Model (Cycle 33)

$7 \quad$ AMIP: The Atmospheric Model W. L. Gates December 1992 Intercomparison Project

The Impact of Horizontal T. J. Phillips L. C. Corsetti Resolution on Moist Processes in the ECMWF Model

S. L. Grotch

January 1993 

Radiative Forcing

G. L. Potter

J. M. Slingo

J.-J. Morcrette

L. Corsetti

10

\author{
The Use of General Circulation \\ Models in Detecting Climate \\ Change Induced By \\ Greenhouse Gases
}

11 Preliminary Validation of the Low Frequency Variability of Tropospheric Temperature and Circulation Simulated for the AMIP by the ECMWF Model

12

Simulation of the Indian and East-Asian Summer Monsoon in the ECMWF Model: Sensitivity to Horizontal Resolution

Statistical Intercomparison of Global Climate Models: A Common Principal Component Approach

14 Ocean Variability and its Influence on the Detectability of Greenhouse Warming Signals
B. D. Santer

U. Cubasch

U. Mikolajewicz

G. Hegerl

J. S. Boyle
April 1993

February 1993

March 1993 
15 Cloud-Radiative Effects on Implied Oceanic Energy Transports as Simulated by Atmospheric

D. A. Randall General Circulation Models G. Boer R. Colman M. Dix V. Galin

M. Helfand

J. Kiehl

A. Kitoh

W. Lau

X.-Z. Liang

V. Lykossov

B. McAvaney

K. Miyakoda

S. Planton 